
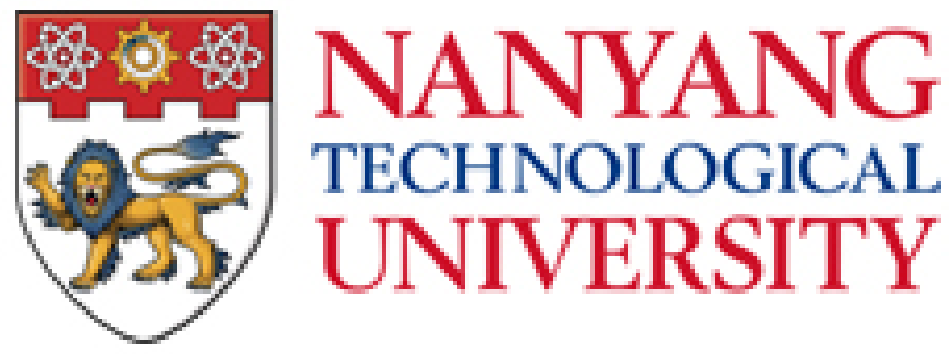

\title{
LOW VELOCITY IMPACT RESPONSE OF HYBRID AND FIBRE-METAL COMPOSITES
}

\section{PERIYASAMY MANIKANDAN}

School of Mechanical and Aerospace Engineering

A thesis submitted to the Nanyang Technological University in fulfilment of the requirement for the degree of

Doctor of Philosophy 


\section{Acknowledgment}

First and foremost, I would like to thank my supervisor Dr. Chai Gin Boay for providing this memorable opportunity to work under him. I express my sincere gratitude for his continuous support, patience, motivation, immense knowledge and belief to my research work.

Besides, I take this moment to sincerely acknowledge Nanyang Technological University (NTU) for providing financial assistance in the form of graduate scholarship which buttressed me to perform my research work more efficiently and in comfort. In regards to the experimental work, I thank the technical staffs of Aircraft structures lab, Material lab -1 and 2, Mechanics of material lab, undergraduate and master students for their support. I extend my thanks to Dr. Leong Yew Wei of IMRE to provide facilities to perform impact experiments.

I thank all my fellow lab mates and my surrounding friends for their constant motivation and valuable discussions in terms of technical and personal as well.

Furthermore, I would like to pay high regards to my parents, sister and relatives who always strengthen me mentally to do best in any circumstance.

Finally, I will forever be thankful to my college senior and friend, Dr. S. Nadaraja Pillai who constantly encourage me to pursue a research opportunity abroad. Without him the present life is unimaginable. 


\section{Abstract}

The present thesis covers detailed investigation on the quasi-static indentation and low-velocity impact response of Fibre Metal Laminates (FML) using experimental and finite element simulation study.

The FML panels investigated were made from balanced woven GFRP fabric composite layer bonded with aluminium alloy Al 2024-O metal sheet. Indentation and low velocity impact experiments were conducted on FML panels of $1 / 1,2 / 1$, $3 / 2$ and $4 / 3$ configurations using hemispherical ended indenter/impactor. Static indentation test allows one to correlate the characteristic load-displacement curve with distinct failure stages of the respective material layer in the FML panel. It also provides corresponding energy magnitude required to induce particular failure mode in FML panel. Using these energy levels, impact velocities for impact tests were derived.

Along with experimental observations, a robust and reliable finite element model was developed using ABAQUS, a commercially available finite element software. Adhesive bonding between metal and composite layers of FML was modelled using special purpose cohesive element. Three non-interactive material models were defined to simulate the behaviour of metal, composite and cohesive interface in finite element model. The damage response was defined using continuum damage mechanics (CDM) based damage model constituting 1) Johnson-cook (JC) failure criteria for metal layer 2) Interactive mode based failure model for composite layer and 3) Quadratic nominal stress based failure criteria for cohesive layer in progression with fracture mechanics based damage evolution formulation. High fidelity solutions were 
obtained through these robust finite element models.

Variable parameters having interrelated influencing factors were studied to understand the role of the material layers to indentation and impact response of FML. The influence of initial contact surface (geometry related), impact velocity (event related) and metal composite interface (MCI) bonding (material related) were considered for the investigation. Both experiment and numerical results showed that the considered geometry and the material related parameters have strong influence on the threshold force to cause damage initiation in FML whereas the threshold influence of event related parameter is constant for all investigated FML configurations. From the absorbed energy perspective, FML panels absorb 90\% of impact energy in average through different dissipation modes; $\sim 60 \%$ by plastic deformation of the metal layer, $\sim 20 \%$ by residual elastic energy and $\sim 10 \%$ by forming new damage surface.

Finally, the influence of the clustering level of metal and composite layers towards the structural integrity of FML panel was analyzed. Load carrying capability, plastic flow of metal layers and extent of damage in each material layers were found strongly dependent on level of clustering for a given FML panel thickness and metal volume fraction (MVF). 


\section{Contents}

Acknowledgment i

$\begin{array}{ll}\text { Abstract } & \text { ii }\end{array}$

Table of contents $\quad$ iv

List of Figures ix

List of Tables $\quad$ xiii

Nomenclature $\quad$ xv

$\begin{array}{lll}1 & \text { Introduction } & 1\end{array}$

1.1 Background and Motivation . . . . . . . . . . . . . . . 1

1.1.1 Material revolution - in a historical perspective . . . . . . . . 1

$1.1 .2 \quad$ FRP composites - an impact prone material . . . . . . . . . . 2

1.1.3 Hybridization methods of FRP composites . . . . . . . . . . . 3

1.1.4 Fibre metal laminates (FML) - an optimum damage tolerant hybrid . . . . . . . . . . . . . . . . 4 4

1.2 Research purpose . . . . . . . . . . . . . . . . . . 5

1.3 Research objective . . . . . . . . . . . . . . . . 6

1.4 Research scope . . . . . . . . . . . . . . . . . . . . . . 6

1.5 Layout of the Thesis $\ldots \ldots \ldots \ldots \ldots$. . . . . . . . . . 7

\begin{tabular}{lll}
\hline 2 & Literature review & 9
\end{tabular}

2.1 Impact behaviour of FML $\ldots \ldots \ldots \ldots \ldots$ 
2.2 Review of experimental studies f . . . . . . . . . . . . . . . 11

$2.2 .1 \quad$ Influence of metal layer . . . . . . . . . . . . . . . . . 12

$2.2 .2 \quad$ Influence of FRP layer $\ldots \ldots \ldots \ldots$. . . . . . . . . . . 12

$2.2 .3 \quad$ Influence of Metal volume fraction (MVF) . . . . . . . . . 16

$2.2 .4 \quad$ Impact damage morphology $\ldots \ldots \ldots$. . . . . . . . . 17

2.3 Review of numerical simulation studies . . . . . . . . . . . . . . . 18

$2.3 .1 \quad$ Modeling strategies of FML $\ldots \ldots \ldots$. . . . . . . . . . . 18

2.3 .2 Importance of cohesive elements in FML modelling . . . . . . 21

2.4 Review of analytical studies . . . . . . . . . . . . . . . . . . . 22

$2.4 .1 \quad$ Analytical modelling strategies of impact event . . . . . . . . 22

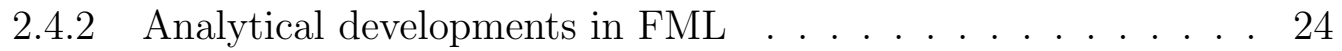

2.5 Conclusion . . . . . . . . . . . . . . . . . . . . . . . . 28

\begin{tabular}{|lll}
\hline 3 & Test configurations and constitutive material models & 30
\end{tabular}

$3.1 \quad$ Experimental configurations . . . . . . . . . . . . . . . . . . 30

$3.1 .1 \quad$ Quasi-Static Indentation (QSI) test . . . . . . . . . . . . . 30

3.1 .2 Low velocity impact (LVI) test . . . . . . . . . . . . . . 31

3.2 Details of FE model $\ldots \ldots \ldots$. . . . . . . . . . . . . . . . . 34

$3.3 \quad$ Material model for the metal layer . . . . . . . . . . . . . . . . . . . . 37

3.3 .1 Details of constitutive model . . . . . . . . . . . . . . . . . . 37

3.3 .2 Calibration of material parameters . . . . . . . . . . . . . 39

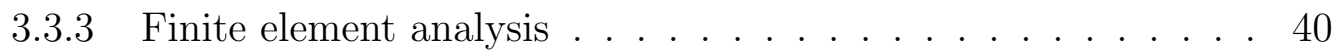

3.3.3.1 Indentation response of bare aluminium plate . . . . 40

3.3.3.2 Low velocity impact response of bare aluminium plate 41

3.4 Material model for FRP composite layer . . . . . . . . . . . . . . . . 42

3.4 .1 Details of constitutive model . . . . . . . . . . . . . . . . . . 42

3.4 .2 Calibration of material parameters . . . . . . . . . . . 46

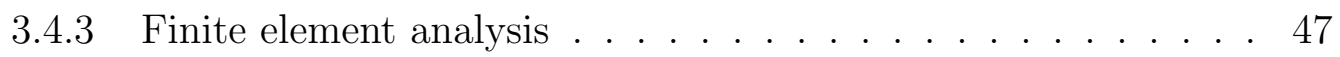

3.4.3.1 Indentation response of woven FRP composite plate 47

3.4.3.2 Impact response of woven FRP composite plate . . . 48 
3.5 Material model for interlaminar region . . . . . . . . . . . . . . . . . 49

3.5 .1 Constitutive behaviour . . . . . . . . . . . . . . 50

3.5 .2 Calibration of constitutive model . . . . . . . . . . . . . . 51

3.5.2.1 Penalty stiffness . . . . . . . . . . . . . . 51

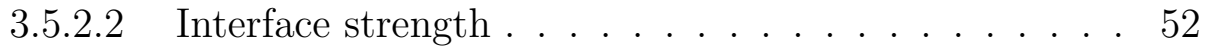

$3.5 .2 .3 \quad$ Critical fracture toughness $\ldots \ldots \ldots$. . . . . . . . 52

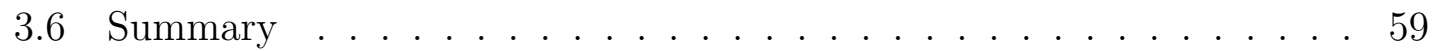

4 Impact experiments on transparent plastics - A similitude model $\begin{array}{ll}\text { for FML } & 60\end{array}$

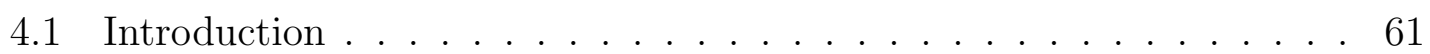

$4.2 \quad$ Experiment configuration and structure . . . . . . . . . . . . . . . . 62

4.3 Impact response of transparent plastics . . . . . . . . . . . . . . . . 63

4.3 .1 Contact force Vs impactor displacement . . . . . . . . . . . 63

4.3 .2 Absorbed energy Vs impactor displacement . . . . . . . . . 65

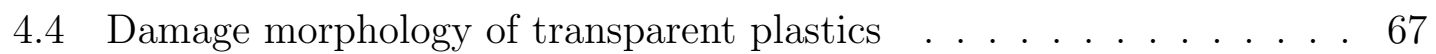

4.5 Summary of conclusion . . . . . . . . . . . . . . . . . . . 70

5 Role of material constituents in FML 72

5.1 Introduction . . . . . . . . . . . . . . . . . . . . . 72

5.2 Test structure . . . . . . . . . . . . . . . . . . . . . . . . . . . 73

$5.2 .1 \quad$ Materials and Fabrication procedure $\ldots . . . . . . .73$

5.2 .2 Test configurations . . . . . . . . . . . . . . . 74

$5.3 \quad$ Indentation and impact behaviour of FML-1/1 panel . . . . . . . . . 75

5.3 .1 Influence of initial contact surface . . . . . . . . . . . . . . 75

5.3.1.1 Observed experimental response . . . . . . . . 76

5.3.1.2 $\quad$ Predicted numerical response . . . . . . . . . . . 79

5.3 .1 .3 Effect of MVF . . . . . . . . . . . . . . . 81

$5.3 .1 .4 \quad$ Layer-wise energy partition . . . . . . . . . . . . . 82

$5.3 .1 .5 \quad$ Mode-wise energy partition . . . . . . . . . . 84 
5.3 .2 Influence of impact velocity $\ldots \ldots \ldots \ldots$. . . . . 86

5.3.2.1 Quasi-static indentation response . . . . . . . . . . 86

$5.3 .2 .2 \quad$ Low velocity impact response $\ldots \ldots \ldots$. . . . . . . 89

$5.3 .3 \quad$ Influence of MCI bonding . . . . . . . . . . . . . . . . . 95

5.3.3.1 Response of soft side tested sample . . . . . . . . . . 97

$5.3 .3 .2 \quad$ Response of hard side tested sample $\ldots . . . .99$

5.4 Indentation and impact behaviour of FML-2/1 panel . . . . . . . 102

5.4 .1 Influence of impact velocity $\ldots \ldots \ldots \ldots$. . . . . . . 102

5.4.1.1 Quasi-static indentation response . . . . . . . . . . 103

5.4 .1 .2 Low velocity impact response . . . . . . . . . . . . 104

5.4 .2 Influence of MCI bonding . . . . . . . . . . . . . . . 109

5.4.2.1 Quasi-static indentation response . . . . . . . . . . 109

5.4 .2 .2 Low velocity impact response . . . . . . . . . . . . 112

5.5 Summary $\ldots \ldots \ldots \ldots \ldots \ldots$

\begin{tabular}{|ll|}
6 & Structural Integrity of Fibre Metal Laminates \\
\hline
\end{tabular}

6.1 Introduction . . . . . . . . . . . . . . . . . . . . . . . 117

6.2 Test configuration . . . . . . . . . . . . . . . . . . 118

6.3 QSI test on FML panels $\ldots \ldots \ldots \ldots$

$6.3 .1 \quad$ Experimental results . . . . . . . . . . . . . . . . . 120

6.3 .2 Numerical results . . . . . . . . . . . . . . . . . . . . 122

$6.3 .3 \quad$ Damage characterization under QSI loading . . . . . . . . 124

$6.4 \quad$ LVI test on FML panels $\ldots \ldots \ldots \ldots$

$6.4 .1 \quad$ Experimental and Numerical results . . . . . . . . . . . . 126

$6.4 .2 \quad$ Damage characterization under LVI loading . . . . . . . . . 129

6.5 Influence of ply clustering - an interply hybridization concept. . . . . 130

6.6 Summary $\ldots \ldots \ldots \ldots \ldots$

7 Conclusions and future work 133

7.1 Concluding remarks $\ldots \ldots \ldots \ldots$ 
7.1.1 Original contributions in this research . . . . . . . . . . . 136

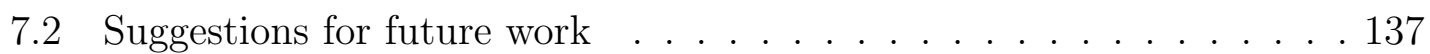

7.3 List of Publications . . . . . . . . . . . . . . . . . . . . . . . . 138

\begin{tabular}{lr}
\hline References & 140
\end{tabular}

\begin{tabular}{|ll}
\hline A Selection of cohesive analysis parameters & 156
\end{tabular}

A.1 Interfacial strength modification strategy . . . . . . . . . . . . . . 156

A.2 Analysis of Mode-I progressive delamination growth . . . . . . . . . . 158 


\section{List of Figures}

$1.1 \quad$ Impact Prone zones on a typical passenger aircraft . . . . . . . . . . . . 2

1.2 Damage morphology of flat target plate indented by blunt impactor . 4

2.1 Damage tolerance assessment of an impact event on composite material 10

2.2 Typical impact response curves . . . . . . . . . . . . . . . . . . . . . 11

2.3 Parameters that influence the indentation and impact response of FML 11

2.4 Different damage morphology of impacted FML panels . . . . . . . . 17

2.5 Three scale levels of composite material modeling . . . . . . . . . . . 20

2.6 Typical flowchart for simulating continuum damage modeling of a discrete finite element . . . . . . . . . . . . . . . . . . . . . . . . . 21

$2.7 \quad$ Plate response under impact loading $\ldots \ldots \ldots$. . . . . . . . . . . 23

2.8 2-DOF spring mass system for modeling low velocity impact phenomenon . . . . . . . . . . . . . . . . . 25

2.9 1-DOF spring mass system for modeling low velocity impact phenomenon . . . . . . . . . . . . . . . . . . . . 27

3.1 Typical Quasi-static indentation test apparatus . . . . . . . . . . 31

3.2 Typical Low velocity drop weight impact test apparatus . . . . . . . . 32

3.3 Numerical model setup for QSI and LVI FE simulation . . . . . . . . 35

3.4 Typical constitutive response of metal layer . . . . . . . . . . . . . . 38

3.5 Plastic stress-strain curve of different thickness aluminium 2024-O sheet 39

$3.6 \quad$ QSI response of bare aluminium plate of different thickness . . . . . . 41

3.7 Variation of equivalent plastic strain of indented metal plate . . . . . 42 
$3.8 \quad$ LVI response of bare aluminium plate . . . . . . . . . . . . . . . . . . 43

3.9 Constitutive material parameters of FRP layer . . . . . . . . . . . . . 43

3.10 QSI response of monolithic FRP plate . . . . . . . . . . . . . . . . 48

3.11 LVI response of monolithic FRP plate . . . . . . . . . . . . . . . . . . 49

3.12 Constitutive material input parameters of cohesive interface layer . . 51

3.13 Mode-I DCB fracture test . . . . . . . . . . . . . . . . . 54

3.14 Mode-I fracture test with homogeneous FRP substrate . . . . . . . . 55

3.15 Mode-I DCB fracture test sample with dissimilar substrates . . . . . 56

3.16 Mode-I fracture test with heterogeneous FRP substrate . . . . . . . . 57

4.1 Schematic diagram of experimented bi-layered plastic samples . . . . 62

4.2 Force-deflection curve of PMMA-PC plastic laminates . . . . . . . . . 64

$4.3 \quad$ Energy-deflection curve of PMMA-PC plastic laminates . . . . . . . . 66

4.4 Damage patterns of impacted PMMA/PC plastic laminates. . . . . . 68

4.5 Cross sectional photography showing delamination of transparent plastics . . . . . . . . . . . . . . . . . . . . . 69

5.1 Flowchart of test carried to understand FML behaviour . . . . . . . . 74

5.2 Different configuration of FML panel . . . . . . . . . . . . . . . . 75

$5.3 \quad$ Impact damage surfaces of FML-1/1 panel having different MVF. . . 76

5.4 Impact characteristic curves of FML-1/1 panel having different MVF 78

$5.5 \quad$ Experimental and numerical impact response of FML-1/1-MVF=0.5. $\quad 80$

$5.6 \quad$ Deformation profile of soft side impacted FML-1/1 panel . . . . . . . 82

$5.7 \quad$ Layer-wise energy partition of impacted FML-1/1 panel . . . . . . . . 83

5.8 Mode-wise energy partition of impacted FML-1/1- MVF 0.5 panel . . 84

5.9 Indentation and impact response of soft and hard side impacted FML$1 / 1$ panel $\ldots \ldots \ldots$. . . . . . . . . . . . . . . . . . . . . . 88

5.10 Experimental and numerical contact force histories of soft side impacted FML-1/1 panel . . . . . . . . . . . . . . . . . . . . . . . . . . 89 
5.11 Experimental and numerical contact force histories of hard side impacted FML-1/1 panel . . . . . . . . . . . . . . . . . . . . . . . . . . 90

5.12 Experimental and numerical damage surface of soft side impacted FML-1/1 panel . . . . . . . . . . . . . . . . . . . . . . . . . . 90

5.13 Experimental and numerical damage surface of hard side impacted FML-1/1 panel . . . . . . . . . . . . . . . . . . . . . . . . . . 91

5.14 Damage evolution history of soft side impacted FML-1/1 panel. . . . 92

5.15 Damage evolution history of hard side impacted FML-1/1 panel . . . 93

5.16 Energy partition of impacted FML-1/1 panel with different impact velocity . . . . . . . . . . . . . . . . . . . . . . . 994

5.17 Phenomenon illustrating MCI de-bond nucleation by Mode-II fracture mode ........................ 95

5.18 Phenomenon illustrating MCI de-bond nucleation by Mode-I fracture mode . . . . . . . . . . . . . . . . . . 996

5.19 Cross section of two modes of MCI de-bond damage . . . . . . . . . . 96

5.20 Indentation and impact response of self and pseudo-bonded soft side impact FML-1/1 panel . . . . . . . . . . . . . . . . . . . . . . . . . . 98

5.21 Experimental impact damage surface of self and pseudo-bonded soft side impact FML-1/1 panel $\ldots$. . . . . . . . . . . . . . . . . . . . . . 98

5.22 Indentation and impact response of self and pseudo-bonded hard side impact FML-1/1 panel . . . . . . . . . . . . . . . . . . . . . . . . 100

5.23 Experimental impact damage surface of self and pseudo-bonded hard side impact FML-1/1 panel . . . . . . . . . . . . . . . . . . . . 101

5.24 Indentation response of FML-2/1 panel . . . . . . . . . . . . . . . . . 103

5.25 Impact response of FML-2/1 panel . . . . . . . . . . . . . . . . . . . 104

5.26 Impact damage surface of FML-2/1 panel . . . . . . . . . . . . . . . 105

5.27 Experimental and numerical contact force histories of FML-2/1 panel 106 5.28 Numerically predicted MCI de-bond damage surface of FML-2/1 panel 107 5.29 Damage evolution history of impacted FML-2/1 panel . . . . . . . . . 108 
5.30 Energy partition of impacted FML-2/1 panel with different impact velocities . . . . . . . . . . . . . . . . . . . . . . 109

5.31 Indentation response of self and pseudo-bonded FML-2/1 panel . . . 110

5.32 Experimental indentation damage surface of self and pseudo-bonded FML-2/1 panel . . . . . . . . . . . . . . . . . . . . 111

5.33 Impact response of self and pseudo-bonded FML-2/1 panel . . . . . . 112

5.34 Damage surface of impacted self and pseudo-bonded FML-2/1 panel. 113

6.1 Stacking geometry of GLARE panels utilized for QSI and LVI experiments; GLARE (a) 2/1 (b) 3/2 (c) 4/3 . . . . . . . . . . . . . . . . . 119

6.2 Experimental results from QSI tests. (a) Load-deflection curve, (b) Energy absorbed at different damage level . . . . . . . . . . . . . . 120

6.3 Comparison of $P-\delta$ curve from experiment and FE simulation. (a) 2/1 (b) $3 / 2$ (c) $4 / 3 . \ldots \ldots \ldots \ldots \ldots \ldots$

6.4 Plastic energy absorbed by each aluminium layer of FML in QSI simulation . . . . . . . . . . . . . . . . . . . . 123

6.5 Damage morphology of FML panel due to QSI load . . . . . . . . . . 124

6.6 Microscopic images illustrating de-bonding of MCI (a) 2/1 (b) 3/2. 125

6.7 Numerical predictions of MCI de-bond boundaries by cohesive elements 126

6.8 Contact load-impactor displacement curves for (a) 2/1 (b) 3/2 (c) 4/3 127

6.9 Plastic energy absorbed by each aluminium layer of FML in LVI simulation . . . . . . . . . . . . . . . . . . . . . . . 128

6.10 Post impact Deflection profile of FML panel . . . . . . . . . . . . . . 128

6.11 Damage morphology of FML panel due to LVI load . . . . . . . . . . 130

$7.1 \quad$ Failure sequence of FML to indentation and impact load. . . . . . . . 135

A.1 Geometry parameters of cohesive element necessary to model crack propagation ........................... 157

A.2 Load-displacement curve of DCB sample with different mesh size. . . 159 


\section{List of Tables}

1.1 Advantages of FML over monolithic metal . . . . . . . . . . . . . . . 5

1.2 Design strategies of an impact resistant material . . . . . . . . . . . . 6

2.1 Summary of experimental studies on impact response of FML . . . . 14

2.2 Summary of numerical studies on impact response of FML . . . . . . 19

2.3 Clamped circular flexural profile shapes for large deflection analysis of a plate . . . . . . . . . . . . . . . . . . . 24

2.4 Analytical solution methods for FMLs . . . . . . . . . . . . . . . . 24

2.5 Governing Differential Equations of Motion and initial conditions . . 27

3.1 Specifications of target sample and impactor utilized in the experimental study . . . . . . . . . . . . . . . . . . . . 34

3.2 Material properties of AL-2024-O metal sheet . . . . . . . . . . . . . 40

3.3 Material properties of Hexply 7781/L-530 GFRP prepeg . . . . . . . 47

3.4 Summary of predicted fracture energy values . . . . . . . . . . . . 58

3.5 Properties of metal composite interface cohesive layer . . . . . . . . . 59

$4.1 \quad$ Experimental structure to investigate acrylic/PC plastics . . . . . . . 63

$4.2 \quad$ Basic mechanical properties of transparent plastic sheets . . . . . . . 63

$5.1 \quad$ Stacking configuration of FML panels used in indentation and impact

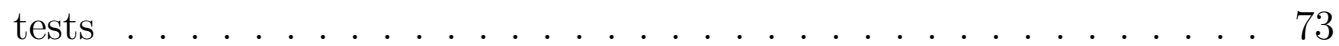

5.2 Configurations used to study influence of initial contact surface of FML-1/1 panel . . . . . . . . . . . . . . . . . 76 
5.3 Impact characteristics of FML-1/1 panel with different MVF . . . . . 81

5.4 Numerical predictions of different modes of energy dissipation . . . . 85

5.5 Configurations used to study influence of initial contact surface of FML-1/1 panel . . . . . . . . . . . . . . . . . . . . . . 86

5.6 Configurations used to study influence of MCI bonding of FML-1/1 panel . . . . . . . . . . . . . . . . . . . . . . . . . 97

5.7 Configurations used to study influence of impact velocity of FML-2/1

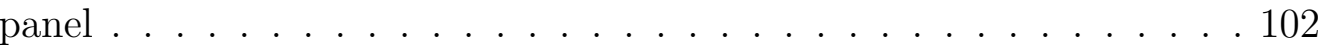

5.8 Configurations used to study influence of MCI bonding of FML-2/1 panel . . . . . . . . . . . . . . . . . . . . . 110

5.9 Numerical predictions of different modes of energy dissipation . . . . 114

$6.1 \quad$ Experimental structure . . . . . . . . . . . . . . . . . . . . . . . . 119

$6.2 \quad$ Energy characteristics of indented samples . . . . . . . . . . . . . . 121

6.3 Average characteristic parameters of FML panels subjected to impact energy $\left(\mathrm{E}_{\mathrm{i}}\right)$ of $34 \mathrm{~J} \ldots \ldots \ldots$

A.1 Adjusted normal interfacial strength with respect to different mesh 


\section{Nomenclature}

Symbols used in the expressions are explained in detail immediately after their first appearance. Nevertheless, some general symbols and common abbreviation used in this report are summarized below.

\section{Symbols}

1,2,3 Material co-ordinate system with 1, 2 - direction indicate fibre orientation

$\alpha \quad$ Indentation depth

$\delta_{\alpha}^{0} \quad$ Relative displacement at the onset of damage

$\delta_{\alpha}^{f} \quad$ Relative displacement at complete failure

$\epsilon_{e q}^{p} \quad$ Equivalent plastic strain

$\langle x\rangle \quad$ McCauley operator

$\omega_{i} \quad$ Directionally dependent internal damage variable

$\phi_{\alpha} \quad$ Loading function required to cause damage

$\epsilon_{i j} \quad$ Strain rate

$\boldsymbol{\epsilon}_{\boldsymbol{i j}}^{\boldsymbol{e}} \quad$ Elastic part of strain $\boldsymbol{\epsilon}_{\boldsymbol{i j}}$

$\epsilon_{i j}^{p} \quad$ Plastic part of strain $\epsilon_{i j}$

$\boldsymbol{\epsilon}_{\boldsymbol{i j}} \quad$ Strain components in material coordinates 


\begin{tabular}{|c|c|}
\hline$\sigma_{i j}$ & Stress components in material coordinates \\
\hline$t_{\alpha}^{c}$ & Critical traction vector \\
\hline$t_{\alpha}$ & Traction vector \\
\hline$\sigma_{y}$ & Yield stress \\
\hline$\tilde{\sigma}$ & Effective stress \\
\hline$C(\omega)$ & Damaged elasticity or stiffness matrix \\
\hline$C_{i j k l}$ & Undamaged Elasticity or Stiffness matrix \\
\hline$E(t)$ & Energy history \\
\hline$E_{a}$ or $E_{a b s}$ & Absorbed energy \\
\hline$E_{i}$ & Impact or Incident energy \\
\hline$E_{r}$ & Rebound energy \\
\hline$F_{\alpha}$ & Damage activation function \\
\hline$g_{f}^{\alpha}$ & Fracture energy per unit area \\
\hline$G_{\alpha}^{c}$ & Critical fracture toughness \\
\hline$G_{\alpha}$ & Fracture toughness or Strain energy density \\
\hline$h$ & Vertical drop height \\
\hline$k_{c}$ & Modified Hertz contact stiffness \\
\hline$L_{c}$ & Characteristic element length \\
\hline$M_{0}$ or $m$ & Mass of impactor \\
\hline$m_{e q}$ & Effective plate (target) mass \\
\hline$P(t)$ & Contact force history \\
\hline
\end{tabular}




\begin{tabular}{|c|c|}
\hline$P_{\max }$ & Maximum contact force \\
\hline$r_{\alpha}$ & Elastic domain threshold \\
\hline$S_{L}$ & In-plane shear strength \\
\hline$S_{T}$ & Out of plane shear strength \\
\hline$S_{i j k l}$ & Compliance matrix \\
\hline$t_{c}$ & Contact time \\
\hline$v(t)$ & Velocity history \\
\hline$V_{i}$ & Impact velocity \\
\hline$w_{0}$ & Center deflection of the target \\
\hline$w_{m}$ & Maximum displacement of impactor \\
\hline$w_{t=t c}$ & Position of impactor at which contact event ends \\
\hline$x(t)$ & Displacement history \\
\hline$X_{+}$ & Tensile strength in 1- direction \\
\hline$X_{-}$ & Compressive strength in 1 - direction \\
\hline$Y\left(\epsilon_{e q}^{p}\right)$ & Material point yielding or hardening \\
\hline$Y_{+}$ & Tensile strength in 2- direction \\
\hline$Y_{-}$ & Compressive strength in 2 - direction \\
\hline
\end{tabular}

Acronyms

ARALL Aramid Reinforced ALuminium Laminate

BVID Barely Visible Impact Damage

CAI Compression After Impact 
CARALL CArbon Reinforced ALuminium Laminate

CDM Continuum Damage Mechanics

CP Complete Perforation

DD

Dent Damage

FC

First Crack

FEM

Finite Element Model

FML

Fibre Metal Laminate

FRP

Fibre Reinforced Polymer

GLARE

GLAss REinforced aluminium laminate

LVI

Low Velocity Impact

MCI

Metal Composite Interface

MVF

Metal Volume Fraction

QSI

Quasi Static Indentation

TTT

Through The Thickness

UD

Uni-Directional

VUMAT Vectorized User-defined MATerial subroutine 


\section{Chapter 1}

\section{Introduction}

\subsection{Background and Motivation}

\subsubsection{Material revolution - in a historical perspective}

Regardless of the domain, two major fields need to be in its optimum level behind every invention for prolonged sustainable life: Economy and Ecology. To achieve that, it is necessary to consider three important factors during the design phase; Weight, Energy and Cost.

So far, manufacturing industries confronted two material revolutions. The first revolution dated back to the $19^{\text {th }}$ century, during which the structures made of wood and ceramics were substituted with metal alloys. However, high density and sensitiveness to corrosion impede their continuous procurement. This resulted to second revolution around 1940's during which the metal made structures were partially or completely replaced with an incredibly light material called Fibre Reinforced Polymer (FRP) composites. Historically, this development was incubated majorly during World War II (1940) by the military and defence sectors that required less weight structures for economical transportation. Soon after, the developments in FRP technology hit the global markets of multiple industrial sectors like Aviation, Automobile, Marine, Oil and gas, Wind turbines, Infrastructure etc. Despite the dominance of FRP material, metals like Aluminium and steel are still being use in primary load carrying structures. As one Boeing engineer stated in a recent Aviation 


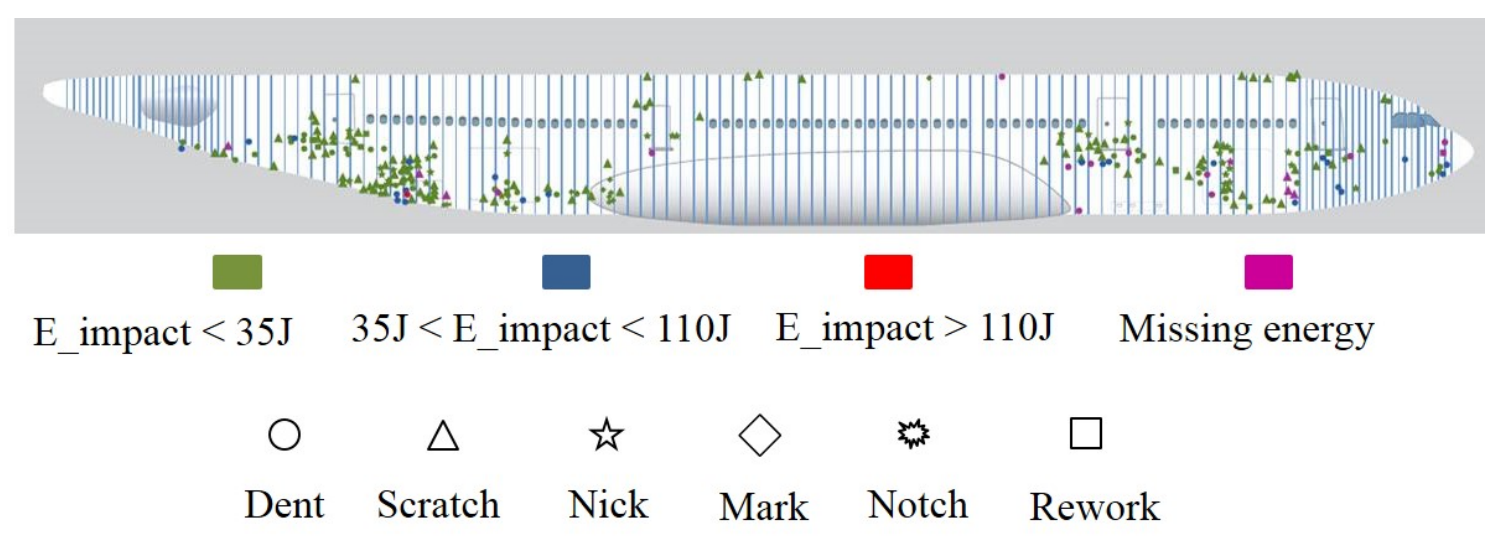

Figure 1.1: Impact Prone zones on a typical passenger aircraft 3

Consumer article that: "The more we learn about composites, the better aluminium looks". Brittle nature and high manufacturing cost may be the major stumbling blocks in the use of FRP in primary structures.

\subsubsection{FRP composites - an impact prone material}

Susceptibility to damage from the quasi-static indentation (QSI) and dynamic low-velocity impact (LVI) force is one of the major design concerns for FRP structures because significant damage could result but yet undetectable by visible inspection [1].

Upon application of contact load, compressive stresses are generated under the contact region and continue to act in the impacted material (target) until the impacting body ( impactor) rebound. Such induced stresses will propagate through the structure with decreasing amplitude and reflected as a tension wave at the back surface of the target with complex distribution of interlaminar shear stress internally [2]. Interlaminar delamination and matrix cracking are the major damage modes of low energy impact $(\sim<35 \mathrm{~J})$ and include fibre damage at higher impact energy level $(\sim>35 \mathrm{~J})$.

Figure 1.1 shows the impact prone regions of typical fuselage section of passenger aircraft. One can realize a current technically advanced passenger air carrier Boeing 787 (popularly known as Dreamliner) which utilized $50 \%$ by weight of composites on its structure and in particular the whole fuselage is made of high strength but brittle CFRP (Carbon FRP) composites. From the failure report of 71 Boeing 747 aircraft 
having 29,500 endurance, it was found 90 out of 688 repairs (13\%) are caused by impact of foreign bodies [4]. Apparently, this fact confirms a higher probability for the occurrence of impact induced damage and thus the designers are to ensure that the structure is impact resistant in the earlier stages of design process.

\subsubsection{Hybridization methods of FRP composites}

Numerous hybridization methods have been attempted to improve the impact resistance of FRP composites with varying degree of success [5]. These include; matrix modifications (rubber toughening and thermoplastic), through the thickness reinforcements (braiding, 3-D weaving, stitching), fibre architecture and usage of high strain fibres. Amongst all, the last technique seems to be more promising since the major cause for the low impact resistance in FRP composites is believed to be due to low failure strain of fibre reinforcements [6].

Studies have shown that there is improved impact damage resistance/tolerance on FRP composites by introducing woven fabric in place of unidirectional (UD) or cross ply fibre reinforcements [7]. The major striking force to provide such advantageous feature by woven fabric is the improved interlaminar fracture toughness [8,9]. In addition, the interlacing of fibres one above the other on mutually perpendicular direction provided greater tolerance to crack growth and significantly lowered the damage area [10] and improved the residual tensile and compression strength after impact [1].

On the other side of material spectrum, metal alloys undergo extensive plastic deformation prior to failure under impact load. The ultimate tensile strain of Aluminium-2024-T3 alloy is approximately 6 and 11 times larger than typical epoxy based GFRP and CFRP composites respectively. The impact resistance of FRP composites might be greatly improved when it is hybridized with metal alloys. The typical response of monolithic and hybrid material subjected to non-perforated punch load by blunt hemispherical impactor are shown in Figure 1.2, It is evident that the intensity and locus of resultant damage surface of FRP layer is greatly attenuated by reinforcing a metal layer as compared between Figure $1.2 \mathrm{~b}$ and $1.2 \mathrm{k}$. 


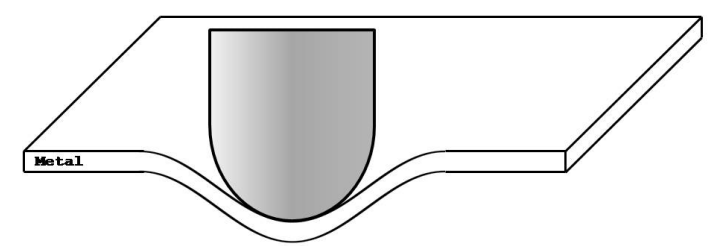

(a) Metal

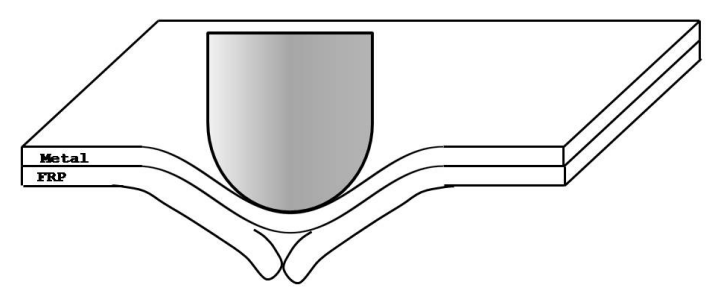

(c) FML-1/1

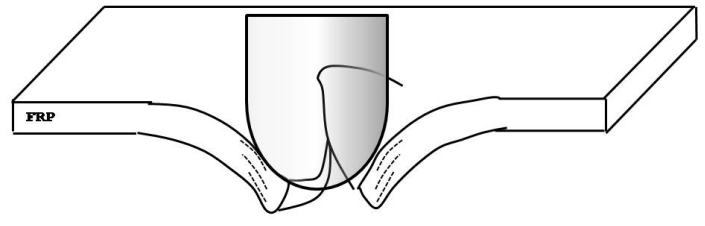

(b) Laminated FRP

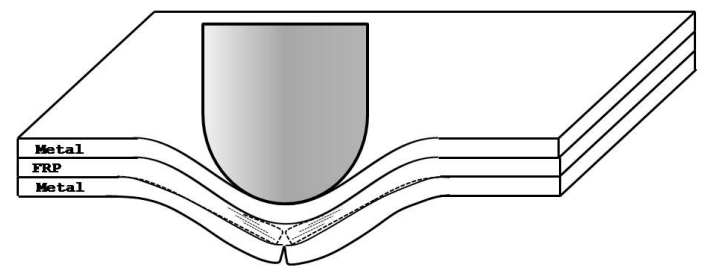

(d) FML-2/1

Figure 1.2: Damage morphology of flat target plate indented by blunt impactor. [Note: FML-A/B represents number of metal/composite layers in the laminate]

\subsubsection{Fibre metal laminates (FML) - an optimum damage tolerant hybrid}

The durability of metal sheets in a structure was found to be greatly enhanced by stack plies of thin bonded layers instead of a single thick layer where adhesive interfaces act as crack stoppers. With extensive developments in adhesive bonding technology, the joining of simultaneous thin metal sheets with FRP layers resulted in a positive hybrid material called Fibre Metal Laminates (FML), a bi-material interlaminar heterogeneous composite developed by Fokker Aerostructures and Delft University around 80's $[12$. This combination provided mutual benefits to both material constituents where the brittle characteristic of FRP composite is complemented by the metal reinforcements and in turn the metal layers attained corrosion protection through FRP layers and trustworthy, an optimum hybrid unveiled enhanced impact and fatigue crack resistance.

The recent successful emergence of Glass Reinforced ALuminium Laminate, wellknown as GLARE, a FML family member housing upper and side skin shells of front and rear portion of Airbus A380 fuselage justifies the potential of FML hybrid concept. The improvements attributed by this hybrid material was found to exploit the extensive plastic flow of metal layer and allow the FRP layer to deform rather than fracture as shown in Figure $1.2 \mathrm{~d}$. The hybrid also showed controlled delamination 
along the interlaminar and interfacial region through intensive fibre bridging.

Unlike a conventional FRP panel where fibre failure would determine the ultimate strength of the structure, stiffness attenuation in FML panels are controlled by various failure combination such as interlaminar delamination between FRP layers, de-bonding between metal composite interface (MCI), fibre breakage and in eventual metal fracture. A summary of salient features of hybrid GLARE FML panel compared to equivalent metal structures are listed in Table.1.1.

These background information motivates to carry out comprehensive research to understand the behavior of fibre metal laminate (FML) under low velocity impact and quasi-static loading conditions.

Table 1.1: Advantages of FML over monolithic metal

\begin{tabular}{lcc}
\hline Property & GLARE & 2024-T3 Aluminium \\
\hline Weight & $0.7-0.9$ & 1 \\
Strength & $1-2$ & 1 \\
Fatigue & $3-100$ & 1 \\
Damage Tolerance & $1-2$ & 1 \\
Impact Blast Resistance & $1-2$ & 1 \\
Flame Resistance & $5-50$ & 1 \\
Lightning Strike & $1.5-2.5$ & 1 \\
Thermal Insulation & $100-150$ & 1 \\
Corrosion Resistance & $1.2-3$ & 1 \\
Reparability & $1+$ & 1 \\
Maintenance & $1+$ & 1 \\
\hline
\end{tabular}

\section{$1.2 \quad$ Research purpose}

Current FML panels consisting of thin sheets of metal layers and unidirectional fibre layers embedded in a adhesive system were originally developed for aircraft structures as a fatigue insensitive material [12]. One specific member from its family called GLARE also expose superior impact damage resistance whose dent depth after impact and residual properties found comparable to aerospace grade aluminium of same areal density [13, 14]. With adequate freedom of material choice, there is ample opportunity to expand this hybrid concept to many other applications where monolithic FRP materials fail to prove its potential. However, elaborate and 
explicit design justification about the reliability of generic FML material as a impact resistant structure doesn't exists. Thus, detailed research plan is still necessary to understand the impact behaviour of this hybrid material concept through extensive experimental and numerical study.

\subsection{Research objective}

The main objectives of the current research work are;

(i) To investigate the structural response of FML subjected to quasi-static indentation and low velocity impact load.

(ii) To establish reliable finite element (FE) models for predicting the indentation and impact response of FML and to estimate the role of each material constituent and metal-composite interface (MCI) to distinct energy absorption phenomenon.

\subsection{Research scope}

As reported by Schrauwen et.al [10], for any structural material designed in view of impact resistance, it must address one or combination of following quantities as listed in Table.1.2. If any material could be tailored to maximum ability of all the below, then it is said to be optimum.

Table 1.2: Design strategies of an impact resistant material

\begin{tabular}{|l|l|}
\hline Energy absorption & $\begin{array}{l}\text { How much of incident energy can the material consume via. } \\
\text { reversible and irreversible deformation and by forming new } \\
\text { damage surfaces? }\end{array}$ \\
\hline Damage resistance & $\begin{array}{l}\text { How durable the structure would be to resist damage initia- } \\
\text { tion for a specified length of time? }\end{array}$ \\
\hline Damage tolerance & $\begin{array}{l}\text { How tolerant the structure would be to resist catastrophic } \\
\text { failure in the presence of damage? }\end{array}$ \\
\hline
\end{tabular}

In a homogeneous material, it is extremely difficult to achieve all the above three and one often needs to sacrifice at least one of them. However, heterogeneous hybrid composite material like Fibre metal Laminates (FML) can be optimized if 
the material behaviour is well understood. This necessitates to assess the role of each material constituents of FML to the resultant indentation and impact response.

The pronounced damage tolerance of existing impact resistant GLARE panel was achieved via controlled delamination enforced by pullout of unidirectional fibres that can bridge the propagating crack front [15]. Exchange of unidirectional fibre prepreg layers by inherently damage tolerant woven glass fabric might be more beneficial however damage resistance is lowered due to reduction in in-plane properties. New research is needed to understand the behaviour and compatibility of woven fabric with metal layers during the indentation and impact process.

The contributions furnished by each layer in a laminated structure to the resultant response is extremely difficult to extract through experimental study. For heterogeneous FML laminate such information is extremely important in order to design the panel with optimum relative volume. With appropriate constitutive material model and failure criterion, high fidelity solutions can be obtained using finite element analysis. It is thus possible to simulate the behaviour of each layer before and after the damage accurately. There is a need to carry out detailed numerical study using reliable finite element model to understand the role of each layer and material in FML.

Structural integrity of heterogeneous unidirectional based FML material is heavily reliant on the thickness of adjacent metal layer and is greatly controlled by intensive fibre bridging phenomenon. With respect to woven fabric FML where bridging of fibres is almost negligible, the research will focus on investigating the influence of layer thickness and strength of metal composite interface (MCI) region towards the integrity of FML under indentation and low velocity impact load.

\subsection{Layout of the Thesis}

The research work presented in this thesis is organized into following chapters. Chapter 2 consists of comprehensive review on indentation and low velocity impact response of FML. The existing loop holes related to the present problem and 
its importance are highlighted in the epilogue. In Chapter 3 , the details regarding experiment set-up, finite element model used for indentation and impact event simulation are presented. Furthermore, details of respective constitutive material model and its reliability based on the calibrated input parameters are also given.

A similitude approach mimicking the role of metal and composite layer in FML to impact behaviour is discussed with transparent plastics in Chapter 4. Chapter 5 dissects the role of material constituents of woven fabric based FML. Extensive experimental and numerical studies are carried to understand the behaviour of each material to indentation and impact load. Chapter 6 investigates the impact behaviour of FML having different stacking configuration. Ply clustering is used as a variable to estimate the structural integrity of hybrid FML panel to indentation and impact load.

Finally, the major conclusions achieved in this research are given in Chapter 7 along with a summary of possible future research developments. 


\section{Chapter 2}

\section{Literature review}

This present chapter attempts to present a comprehensive review of available literatures about out-of-plane deformation of FML in a motive to support further developments. General introduction on the impact studies on FMLs is discussed in Section 2.1. Experiment based research on different influencing factors are described in Section 2.2. Approaches based on finite element model are reviewed and the necessary developments towards its reliability is revealed in Section 2.3. In Section 2.4. the recent developments related to closed form analytical solution methods are discussed.

\subsection{Impact behaviour of FML}

Impact involves the collision of two bodies: the impactor and the target. Upon collision between rigid impactor with deformable target, contact force develops as the impactor indents the target and initiates stress waves which travel both through the thickness and away from the impact point and cause large local and global out of plane displacement. In the worst scenario, severe damage occur under the contact region of the target. Figure 2.1 shows a typical procedure to evaluate impact resistance of aviation composite materials during certification. The whole process is dependent on many characteristic parameters such as limit (threshold) load, ultimate (peak) load, damage size. Thus, for any new material development, prior knowledge about such parameters is a necessary requirement. 


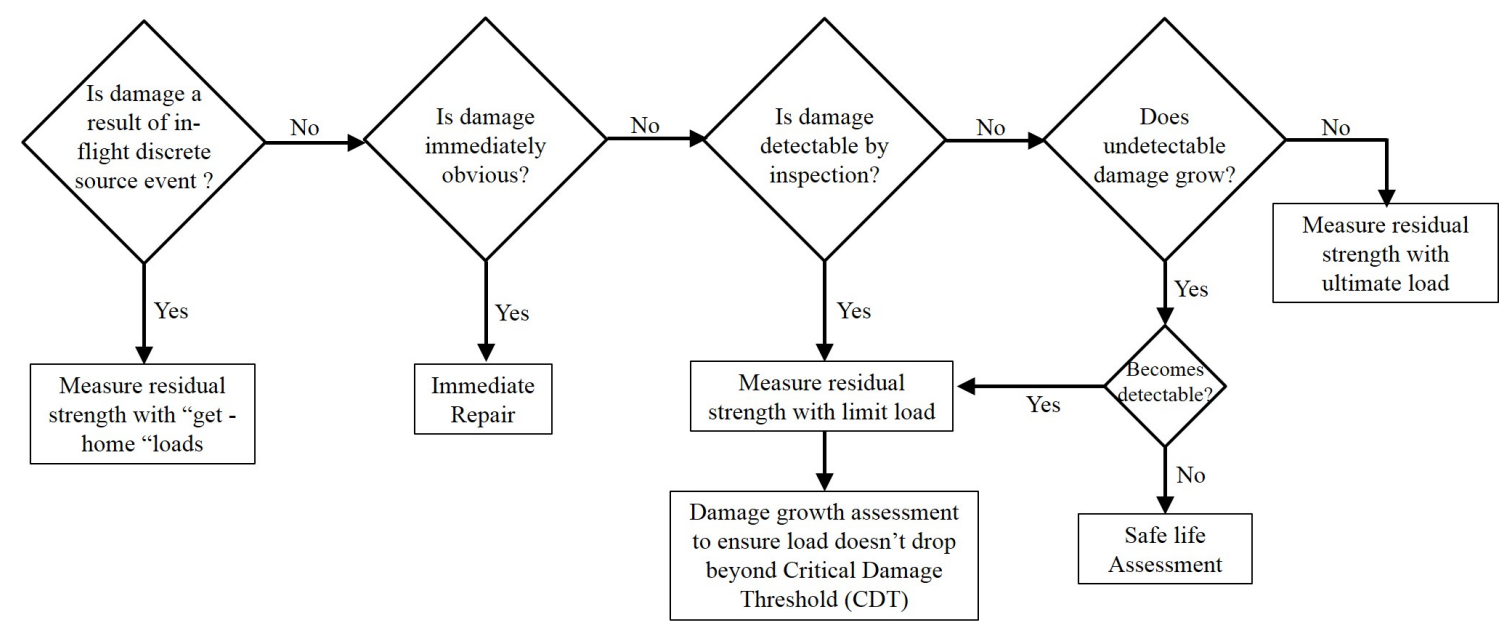

Figure 2.1: Damage tolerance assessment for a typical impact event on composite material in aviation industry. Edited from 16

Within the range of low impact velocity, material characterization from quasistatic indentation and LVI test was found almost similar [17] irrespective of whether target material is strain rate dependent or independent.

The concept of FML constitutes combination of metals and FRP materials. The phenomenon by which metals and FRP materials dissipate the incident kinetic energy during impact is extremely different. Metals essentially absorb energy by elastic and plastic deformation at lower energy level and at higher energy level, they form crack, spall and petal failures. However, the degree of plastic deformation of FRP materials is almost negligible and the energy is almost dissipated by creating new and large damage surfaces. A typical non-perforated impact response curve by metal, FRP and FML material are shown in Figure 2.2. No abrupt drop in contact force exists for metal and FML and even the response is completely smooth for pure metal layers but notable striations exists in FML curve is due to matrix and fibre failure of subsurface FRP layers in FML. In recent years, researchers had made concerted effort to improve the impact behaviour of FRP material through FML concept.

Published research shows that indentation and impact response of FML is dependent on several parameters which are briefly grouped in to three sets [14, 19]: Material based parameters, Geometry based parameters and Event based parameters. Relevant sub-factors corresponds to each governing parameters are categorized in Figure 2.3 
(a)

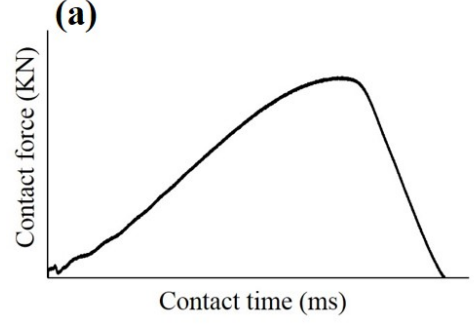

(b)

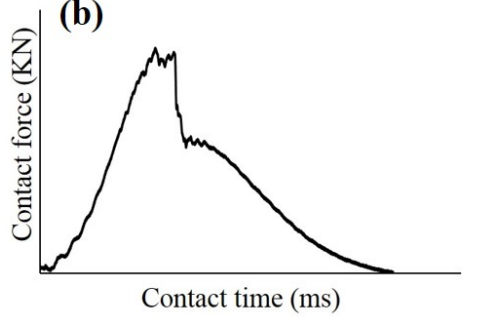

(c)

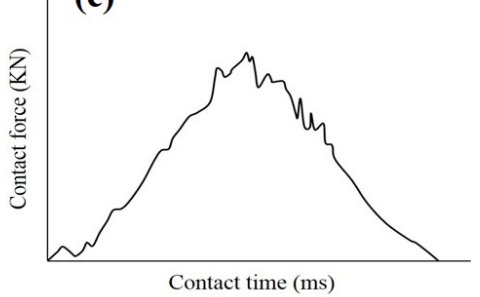

Figure 2.2: Typical impact response curves: (a) metal (b) FRP (c) FML 18

Detailed review of all these factors were briefly presented in the review article [19]. In the succeeding section, pertinent conclusions established through experimental, numerical and analytical methods relevant to current studies present in the thesis are detailed.

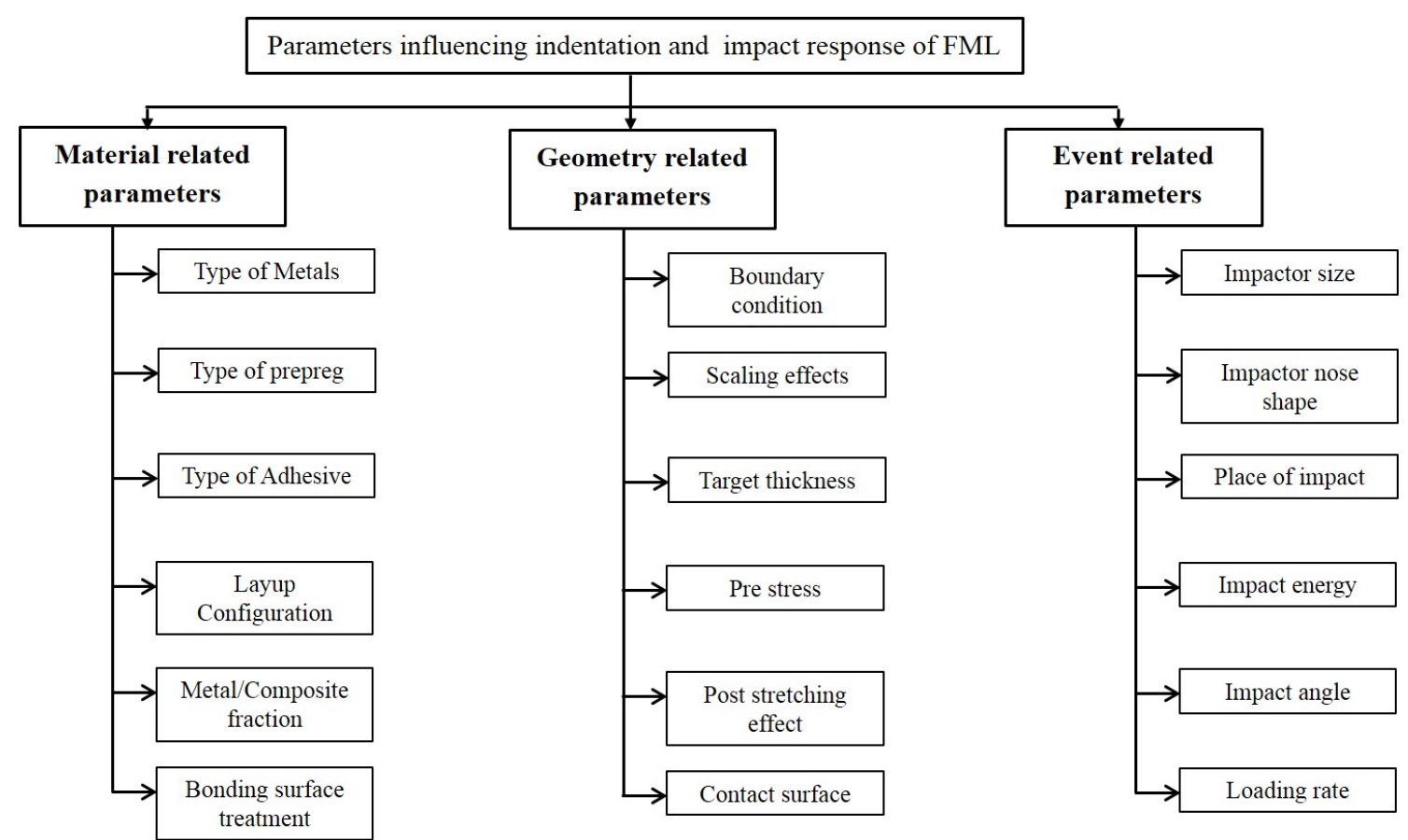

Figure 2.3: Parameters that influence the indentation and impact response of FML

\section{$2.2 \quad$ Review of experimental studies}

Historical developments made through experimental investigation on FMLs under LVI and QSI loading is tabulated in Table 2.1. Discussions on the role of material constituents and resultant damage pattern are highlighted below. 


\subsubsection{Influence of metal layer}

In FMLs, metal dominates the overall performance 20,21] and the influence of different metal alloys on mechanical behaviour is significant. The initial generation of FMLs utilize two Al alloys with varying ductility; 2024-T3 (high fracture toughness) and 7075/7475-T6 (high strength and brittle). FMLs with former alloy absorb energy through plastic deformation whereas energy absorption by fracture mode with latter. Earlier damage initiation recorded for 7000 Al grade FMLs features less favourable damage resistance than Al 2024 alloys 22]. Recently, emphasis had been made to introduce magnesium alloy in FML design for aerospace structures because of its low density, electromagnetic shielding and enhanced corrosive resistance capability. Despite carrying larger damage area and lower specific material properties aside this material option out of auction for primary load carrying structures 23]. Proposal has also been made to use Titanium based FML for high temperature applications [24] but its low ductility yielded poor impact resistance than Al based FML. Overall, ductile nature and reasonable strength are the prime qualities required among metals to deliver better impact resistant FMLs.

\subsubsection{Influence of FRP layer}

The damage pattern and its sequence of occurrence in FML is greatly dependent on types of fibres, fibre architecture, resin and stacking sequence of FRP layer. Aramid, Carbon and Glass fibres got much attention but the first two fail to identify potential applications due to inferior perforation resistance [15]. Eventhough multidirectional fibre orientation delivers improved impact resistance [25], studies concerning different kind of fibre architecture (weaving, braiding) in FML panels was very minimal [26, 27]. There are no comparison data between UD and woven FRP based FML in the literature except reference [27]. Some of the major disadvantages that restrict the use of woven fibres in FML design are less intensity of fibre bridging along the crack plane due to locked fibre weave pattern and reduced tensile, compressive and in-plane shear properties due to fibre crimp compared to 
equivalent UD or cross-ply FRP layer 7 .

Thermoset matrix based FML is advantageous as it has higher strength, stiffness and superior temperature behaviour but weakness side of brittleness, requirement of long processing cycle to ensure complete curing leads to attention towards thermoplastic matrix which require only short processing cycle and offers greater fracture toughness 28 30]. However, the integrity of MCI bond is challenge because of poor adhesion quality of thermoplastic resin with metal surface.

In FMLs, stacking sequence variation is possible either by altering number of metal and FRP layers or altering the fibre orientation of FRP layer in a given number of metal and FRP layer. It has been shown that FML with unidirectional fibre orientation gives the least impact resistance followed by cross-ply and angle ply while superior resistance to impact by quasi isotropic lay-up 21]. It has been shown that crack length and permanent deflection are lower for the panels with fibres in more than one direction and registered larger energy dissipation due to delamination nucleation between dissimilar oriented plies [22]. Fibres oriented in multi-direction showed better impact resistance and damage tolerance also for FML laminates. The damage geometry was found to be sensitive to relative volume fraction of fibre distributed in each direction and large elliptical shape for UD orientation as expected 31-34.

There are few studies comparing FML panels with different number of metal and FRP layers 26,35. However, deductions on optimum configuration for damage resistance and damage tolerance has not been clearly disclosed. But it was advised to use thin metal sheet layers in order to reduce MCI shear stress and thus to limit interface de-bonding [12]. 
Table 2.1: Summary of experimental studies on impact response of FML

\begin{tabular}{|c|c|c|}
\hline Referenc & Year & Observation \\
\hline 13,15 & $1993 / 1996$ & $\begin{array}{l}\text { - Low velocity impact characteristics of GLARE and AR- } \\
\text { ALL FML were determined using drop-weight impact } \\
\text { testing machine. Results have shown GLARE outper- } \\
\text { forms ARALL FML. } \\
\text { - Comparative study between static, low-velocity and high } \\
\text { velocity impact and its corresponding damage resistance, } \\
\text { effect of tensile loading on damage size are some of the } \\
\text { notable scenarios. }\end{array}$ \\
\hline$|36|$ & 1998 & $\begin{array}{l}\text { - Effect of matrix-fibre adhesion to low-velocity impact } \\
\text { on CARALL fibre metal laminates was experimentally } \\
\text { investigated }\end{array}$ \\
\hline$\overline{29}$ & 2000 & $\begin{array}{l}\text { - The enhanced residual strength of FML was noticed } \\
\text { through Impact test and subsequent tensile test }\end{array}$ \\
\hline 37 & 2000 & $\begin{array}{l}\text { - The deterioration of strength due to low-velocity impact } \\
\text { of different stacking sequence ARALL specimens were } \\
\text { evaluated through compression after Impact (CAI) test. } \\
\text { - Despite of having small fibre and micro matric cracks, } \\
\text { the extent of delamination was found severely influences } \\
\text { the residual performance of impacted specimen. }\end{array}$ \\
\hline $\mid 31$ & 2002 & $\begin{array}{l}\text { - Extensive study has been carried out on post impact } \\
\text { fatigue performance of GLARE laminates. It was found } \\
\text { that FML behave superior than isotropic aluminum lam- } \\
\text { inates. }\end{array}$ \\
\hline 32 & 2004 & $\begin{array}{l}\text { - Influence of impact velocity, impactor mass and impact } \\
\text { energy was studied explicitly. Based on the experimental } \\
\text { data, an appropriate semi empirical relations are formu- } \\
\text { lated for different characteristic parameters. }\end{array}$ \\
\hline$\overline{33}$ & 2005 & $\begin{array}{l}\text { - Comparative study of perforation energy between alu- } \\
\text { minum and magnesium based GFPP and CFRP speci- } \\
\text { mens were carried out by conducting low-velocity impact } \\
\text { test. It was concluded that Magnesium based GFPP out- } \\
\text { performs its counterparts. }\end{array}$ \\
\hline$\overline{34}$ & 2005 & $\begin{array}{l}\text { - Impact test were performed on different } 2 / 1 \text { standard } \\
\text { glare specimens. Both NDT and post mortem investiga- } \\
\text { tion were carried out to evaluate the magnitude of dam- } \\
\text { age. From the analysis, Glare } 5 \text { was concluded as a su- } \\
\text { perior impact resistance material than other glare speci- } \\
\text { mens. }\end{array}$ \\
\hline 38 & 2007 & $\begin{array}{l}\text { - Perforation energy of Glare fibre/Aluminum based FML } \\
\text { was calculated at different impact energies. }\end{array}$ \\
\hline
\end{tabular}




\begin{tabular}{|c|c|c|}
\hline 39,40 & $2007 / 2008$ & $\begin{array}{l}\text { - Characterizing the Glare specimens based on the dam- } \\
\text { age stages ranges from plastic dent, BVID, CVID to com- } \\
\text { plete perforation at different impact energies. Residual } \\
\text { strength of these different characteristic candidates was } \\
\text { experimental through post impact fatigue test. }\end{array}$ \\
\hline$\overline{41}, \overline{42}$ & 2008 & $\begin{array}{l}\text { - To minimizing the manufacturing cost for experimenting } \\
\text { the full scale model, scaling effects in the low-velocity } \\
\text { impact response of FML was studied unambiguously. }\end{array}$ \\
\hline 30 & 2008 & $\begin{array}{l}\text { - Relative study between the mechanical properties long } \\
\text { fibre thermoplastic composite (LFT)/ metal laminate } \\
\text { (LML) was carried out. Low velocity experimental shows } \\
\text { LML outperforms LFT. }\end{array}$ \\
\hline 43 & 2009 & $\begin{array}{l}\text { - Glare specimens were placed over high strength Al alloy } \\
\text { substrate which acts as a fatigue crack retarder. Both } \\
\text { low velocity and fatigue test were performed for the above } \\
\text { configured specimens. The fatigue result shows the crack } \\
\text { growth is retarded up to a factor of } 2 \text {. }\end{array}$ \\
\hline 22 & 2009 & $\begin{array}{l}\text { - Effect of material constituents, ply orientation of FML } \\
\text { on low velocity impact was investigated in detail. Com- } \\
\text { pared with unidirectional glare specimens, cross-ply con- } \\
\text { stituents had better impact resistance was the end finding. }\end{array}$ \\
\hline 4 & 2010 & $\begin{array}{l}\text { - To enhance the safety level of mechanical components, } \\
\text { the author interested to investigate carbon based FML. } \\
\text { Both experimental and numerical impact study was per- } \\
\text { formed with descriptive data analysis. }\end{array}$ \\
\hline $4 \overline{45}$ & 2011 & $\begin{array}{l}\text { - Influence of specimen size, impactor diameter, number } \\
\text { of composite layers, metal layers to low velocity impact } \\
\text { was investigated on glare specimens. }\end{array}$ \\
\hline$\overline{48}, \overline{49}$ & $2011 / 2012$ & $\begin{array}{l}\text { - Degradation of material property of glass based FML } \\
\text { due to multiple impacts was studied experimentally. Ul- } \\
\text { timate tensile strength, ductility percentage and failure } \\
\text { strain are the parameters taken for investigation. }\end{array}$ \\
\hline $50 \mid$ & 2011 & $\begin{array}{l}\text { - Effect of different metal constituents and its thickness } \\
\text { on impact response were studied experimentally and val- } \\
\text { idated the obtained data with numerical modeling. }\end{array}$ \\
\hline 25,51 & 2011 & $\begin{array}{l}\text { - Experimental Study was done on glare } 5-3 / 2 \text { specimens } \\
\text { to investigate the effects of different specimen geometry } \\
\text { and lay-up sequence towards the low velocity impact re- } \\
\text { sponse. }\end{array}$ \\
\hline 52 & 2011 & $\begin{array}{l}\text { - Low velocity impact response of glare FML was investi- } \\
\text { gated at different impact velocity and the nature, shape } \\
\text { of the damage were quantified through destructive micro } \\
\text { photography and non-destructive ultrasonic techniques. }\end{array}$ \\
\hline
\end{tabular}




\begin{tabular}{|c|c|c|}
\hline$[\overline{53}]$ & 2012 & $\begin{array}{l}\text { - Impact response of magnesium based FML was deter- } \\
\text { mined through experiments. From the visual inspection, } \\
\text { having a larger shear crack on metal layer with smaller } \\
\text { limit to first failure energy level, it was suspected that } \\
\text { magnesium based FML offer less impact resistance than } \\
\text { aluminium based FML. }\end{array}$ \\
\hline 27 & 2012 & $\begin{array}{l}\text { - A progressive quasi-static indentation test and low- } \\
\text { velocity impact test were carried out to investigate the } \\
\text { impact response of glare specimens. Two different glare } \\
\text { specimens were experimented: Unidirectional and woven } \\
\text { GFRP. Effects of sanding, different layups, impact veloc- } \\
\text { ity were analyzed adequately. Results are validated with } \\
\text { numerical simulation. }\end{array}$ \\
\hline 54 & 2013 & $\begin{array}{l}\text { - Address the influence of impactor mass, ply orientation, } \\
\text { metal thickness, and plate dimension to low-velocity im- } \\
\text { pact response. }\end{array}$ \\
\hline
\end{tabular}

\subsubsection{Influence of Metal volume fraction (MVF)}

Similar to the use of volume fraction of fibres $\left(V_{f}\right)$ and matrix $\left(V_{m}\right)$ to characterize FRP layer, a new parameter called MVF which relates volume fraction of metal over FRP layer is generally used to characterize FML:

$$
M V F=\frac{\sum_{1}^{p} t_{m e t a l}}{t_{F R P}}
$$

where $t_{\text {metal }}$ and $t_{F R P}$ is the thickness of each metal and FRP layers respectively and $p$ represents the number of aluminium layers in the FML panel.

Increasing the MVF will improve the impact performance of FML of given thickness [50] but it will also increase the weight of panel. Thus the selection of optimum MVF for a given thickness is extremely important. From the studies on GLARE-2/1, it was concluded that specific perforation energy of indented and impact samples are almost constant over the range of MVF. The effect of spring back was found to be more pronounced for panel having lower values of MVF. Such phenomenon might develop new MCI debonding surface mainly at the interface near the bottom face of the impacted panels [55]. Decreasing the MVF improves the critical threshold, perforation resistance about three times and delivers smaller damage area [13, 15]. 


\subsubsection{Impact damage morphology}

The failure of FML under low velocity impact loads is dependent on several parameters such as impactor shape (blunt or sharp), support condition of target and impact velocity. The damage modes of FML is highly interactive and complex. Depending on the material layer that fails first, Vlot [15] classified the impact failure of FML as "fibre dominated" and "aluminium dominated" failure. Starikov [20] classified the type of impact damage of FML panels in more explicit fashion as shown in Figure 2.4. (i) Dent damage (DD) permanent indentation along with sub-surface damage on internal FRP layers. (ii) First Crack $(F C)$ dent deformation with crack visibility on non-impacted surface. (iii) Through The Thickness (TTT) crack visible crack surface on both surface with either partial or complete penetration.

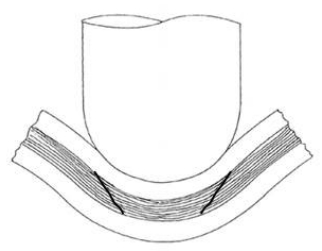

(a)

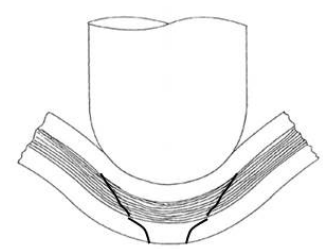

(b)

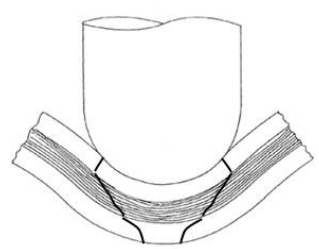

(c)

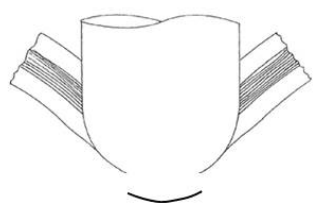

(d)

Figure 2.4: Different damage morphology of impacted FML panels; (a) DD (b) FC (c) TTT (d) Complete perforation. Edited from 12

The overall structural integrity and damage tolerance of impacted FML panel is dependent on the degree of two un-interacted interlaminar failure modes; delamination between laminated FRP plies and interfacial debonding between metal and FRP. Based on the observations of Abrate [56], interlaminar delamination is more obvious between plies with different orientation of UD FRP plies. Meanwhile, debonding is dependent on effectiveness of surface pre-treatment of metal layer and compatibility of adhesives used in MCI region.

The effect of debonding to final impact response is unclear and often assumed interface adhesion strength is stronger than that of interlaminar region [20]. Parnanen [55] exposed that there exists a strong link between MCI debond and adjacent bonded FRP composite failure and insisted detailed characterization study is nec- 
essary for accurate post impact evaluation and for simulating reliable FE model.

\subsection{Review of numerical simulation studies}

It is necessary to have full unambiguous FE model with appropriate interface elements to simulate complete impact failure phenomenon in FML plate structure. The following section provides an overview of the developments of FE modelling made towards the understanding of FML impact behaviour.

\subsubsection{Modeling strategies of FML}

The impact modeling capabilities on FML is still at its infancy. The complexity of physical phenomena including plastic flow in the metal layers, quasi-brittle failure process of FRP layers and existence of interlaminar/interfacial effects are extremely difficult to replicate in the finite element modelling. Research by Hashagen [57] and Linde [58 provide a basic formulation to exploit the use of finite element modeling and techniques for FML and other studies available in literature capitalize the potential of commercial FE codes ABAQUS and LS DYNA. A review of articles devoted to impact behavior of FMLs through commercial FE software are summarized in Table 2.2.

Out of the three different scale of approaches as shown in Figure 2.5, meso-scale modelling gains more interest and was more feasible for large scale simulations [59]. The constitutive models available to define the behaviour for most of the materials in commercial FE software are also formulated compatible with meso-scale. For the case of FML, three separate constitutive models are required to simulate realistic material behaviour; one for metal plasticity, another for composite damage and third for debonding of laminated layers. 
Table 2.2: Summary of numerical studies on impact response of FML

\begin{tabular}{|c|c|c|c|}
\hline Referenc & Year & Software & Observation \\
\hline 60 & 2008 & LS-DYNA & $\begin{array}{l}\text {-Model the low velocity impact on FML along } \\
\text { with user-defined material subroutine based on } \\
\text { continuum damage mechanics (CDM) for compos- } \\
\text { ite layers. } \\
\text { - The post-processing results show delamination is } \\
\text { not a significant damage in low-velocity impact. }\end{array}$ \\
\hline$\overline{61}$ & 2009 & ABAQUS & $\begin{array}{l}\text {-Thermoplastic based FML with elastic-plastic } \\
\text { metal layers and an isotropic composite layer was } \\
\text { simulated. } \\
\text { - Appropriate failure criteria with relevant ele- } \\
\text { ment removal function were defined for both the } \\
\text { metal and composite layer to model a simulation } \\
\text { with high reliability. }\end{array}$ \\
\hline$[44$ & 2010 & ABAQUS & $\begin{array}{l}\text {-Numerical study was carried out to demonstrate } \\
\text { the effect of different impact energies on carbon } \\
\text { based FML using } 2 \text {-D stress based Hashin damage } \\
\text { model for composite layer. } \\
\text { - Metal layer was free from damage criteria be- } \\
\text { cause the author assumes low velocity impact } \\
\text { doesnt produce enough damage due to its high } \\
\text { ductility nature. }\end{array}$ \\
\hline$[\overline{62}]$ & 2010 & ABAQUS & $\begin{array}{l}\text { - Necessity of solid elements over continuum shell } \\
\text { elements, importance of } 3 \mathrm{D} \text { Hashin failure crite- } \\
\text { ria over its } 2 \mathrm{D} \text { form on low-velocity impact were } \\
\text { demonstrated extensively through numerical sim- } \\
\text { ulation. }\end{array}$ \\
\hline 50 & 2011 & ABAQUS & $\begin{array}{l}\text {-Metal types and thickness effect were studied in } \\
\text { numerical and the robustness of the model was } \\
\text { inspected through experimental results. } \\
\text { - Apart from other factors, proper element selec- } \\
\text { tion is dominating to evaluate the accuracy of nu- } \\
\text { merical modeling of FML under low-velocity im- } \\
\text { pact. }\end{array}$ \\
\hline 47 & 2011 & ABAQUS & $\begin{array}{l}\text {-Effect of impactor size, target size and impact } \\
\text { location were studied numerically. } \\
\text { - General contact interaction between the stack- } \\
\text { ing layers, surface-to-surface contact between im- } \\
\text { pactor and target, tensile failure and shear failure } \\
\text { criteria for metal layers, 2D Hashin failure crite- } \\
\text { ria for composite layers are some of the important } \\
\text { facts in this particular simulation. }\end{array}$ \\
\hline 63 & 2011 & ABAQUS & $\begin{array}{l}\text {-An explicit numerical model defining adhesive } \\
\text { layer in between the metal and composite layer, } \\
\text { interface layer using cohesive elements to simulate } \\
\text { interlaminar delamination was developed. }\end{array}$ \\
\hline
\end{tabular}


- External and internal damage effects on each metal face sheets and embedded composite layers were accurately found by computing absorbed energy on each stacking layer.

\begin{tabular}{|c|c|c|}
\hline$[52$ & 2011 LS-DYNA & $\begin{array}{l}\text {-Glass based multilayered FML was modeled us- } \\
\text { ing shell elements and both intra and inter lami- } \\
\text { nar failure was specified using appropriate damage } \\
\text { criteria. } \\
\text { - Contour plots for different composite failure were } \\
\text { obtained which helped to quantify the magnitude } \\
\text { of damage and its severity. }\end{array}$ \\
\hline 27 & ABAQUS & $\begin{array}{l}\text { - A detailed 3D stress based Hashin failure cri- } \\
\text { terion was implemented through subroutine VU- } \\
\text { MAT to capture the damage phenomenon of com- } \\
\text { posites and Johnson cook damage model for metal } \\
\text { layers. }\end{array}$ \\
\hline
\end{tabular}

There are four current approaches available to predict damage of discrete element under impact with intermediate strain rate [64]:

a) Failure criteria approach (dependent on equivalent stress and strain)

b) Plasticity or yield surface approach.

c) Damage mechanics approach (dependent on degradation of material property)

d) Fracture mechanics approach (dependent on energy release rate)

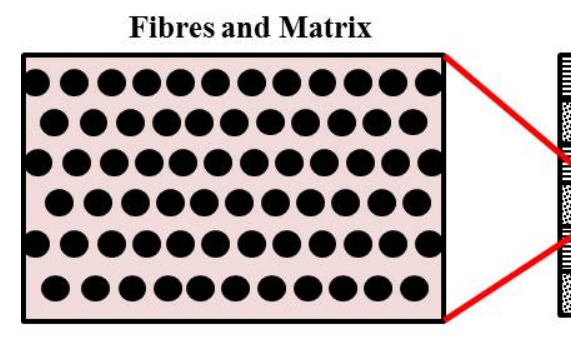

(a) Micro scale

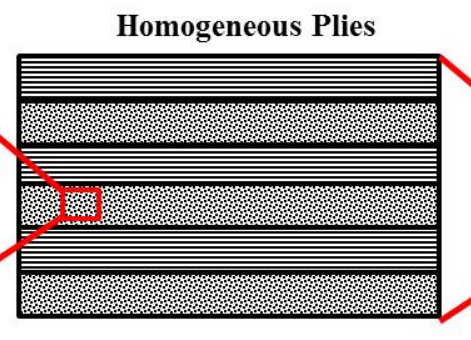

(b) Meso scale
Homogeneous Laminate

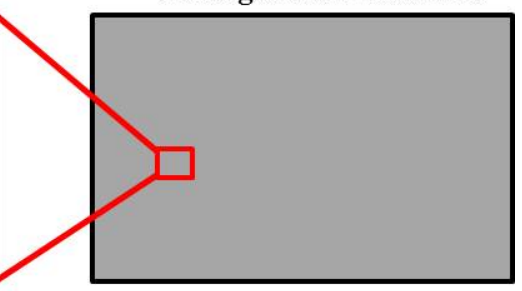

(c) Macro scale

Figure 2.5: Three scale levels of composite material modeling

Numerically, the failure and yield criterion approaches are able to identify failed/plastic yield discrete elements in the FE model. Degradation of element properties are performed based on damage mechanics approach and behaviour of element after damage 
follows fracture mechanics approach. The flowchart in Figure 2.6 depicts the steps involved in modeling damage degradation of discrete element in a typical FE platform. The final damage shapes of each layer can be modelled by removing the failed elements automatically once its stiffness matrix reads null.

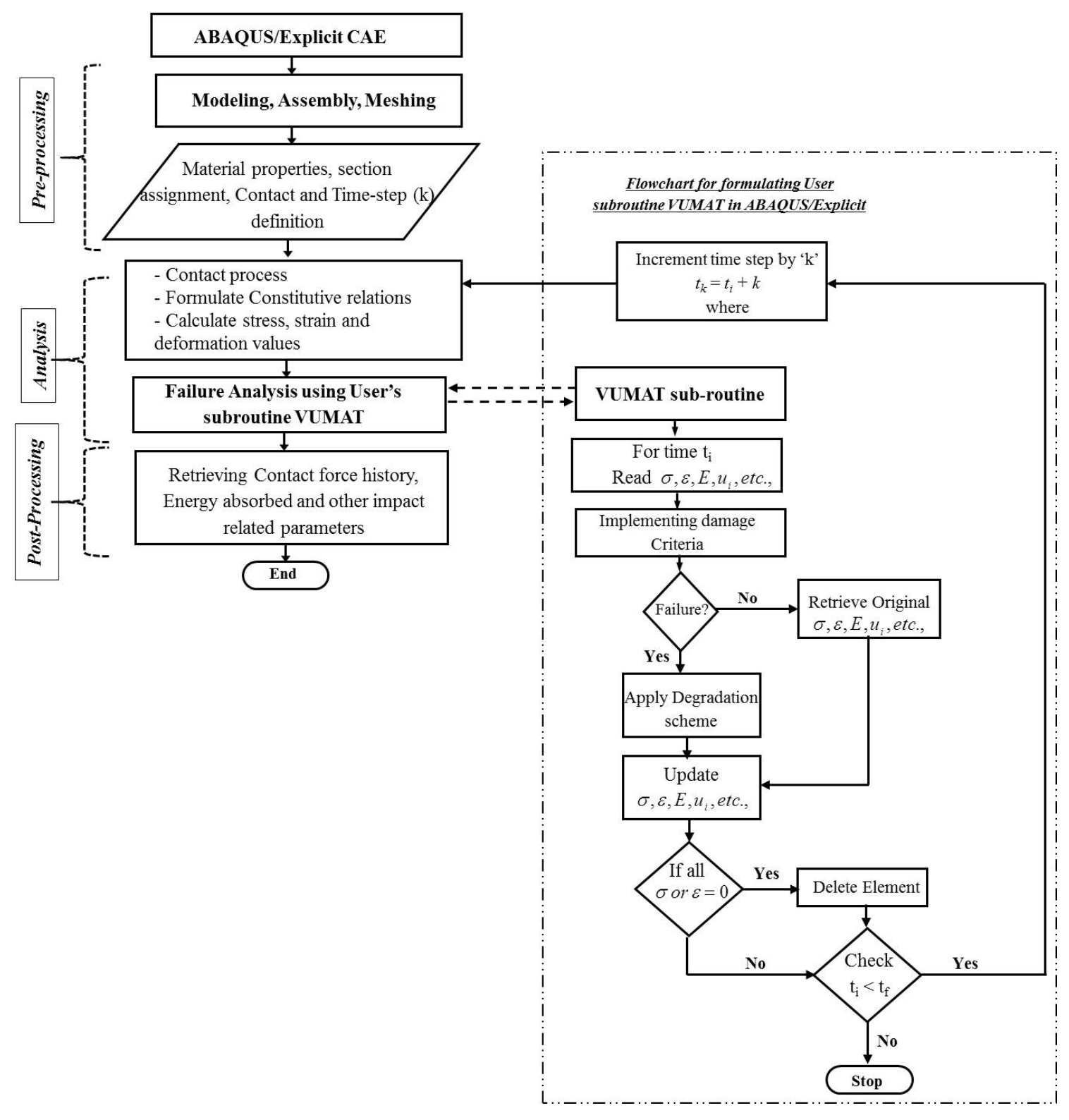

Figure 2.6: Typical flowchart for simulating continuum damage modeling of a discrete finite element

\subsubsection{Importance of cohesive elements in FML modelling}

Cohesive elements in ABAQUS FE platform are special purpose elements used to model regions like adhesive patches, resin rich zones, interfacial layers in composites and some other situations where interfacial strength concept is required. They 
have been used to model the regions which are crack prone, delamination in case of laminated structures etc. The mechanical constitutive behaviour of these elements is governed by different formulations dependent on specific application as follows 65];

- Continuum-based modeling - for adhesives with finite thickness,

- Traction-separation based modeling - for very thin adhesives with almost zero thickness.

Interlaminar and interfacial resin rich regions in FML are very thin and are often considered to be of zero thickness compared to thickness of adjacent material layers. In such cases, it is straightforward to define the constitutive response of cohesive layer in terms of traction-separation.

From the literature knowledge, it is ascertained that most of the available FE works on FML treated the interfacial region as perfectly bonded tie surface and often neglect de-bonding failure mode. Tsartsaris et.al and Laliberte et.al [52,60] used tie-break interface in LS-DYNA and Nakatani et.al [63] used a continuum based model in ABAQUS assuming that the adhesive interface is notably thick. Since, interlaminar and interfacial damage are crucial damage modes on impacted FML panels, it is necessary to implement appropriate interfacial damage formulation to virtually simulate accurate behaviour.

\subsection{Review of analytical studies}

The analytical models are powerful tool to get fast predictive results for a given configuration. This section reviews the available analytical models with focus on indentation and impact behaviour of FML panels.

\subsubsection{Analytical modelling strategies of impact event}

Impact response of thin plates involves both local and global deflection. The resultant damage may be caused by compressive contact stress and/or stress due to structural bending response. Typical deflection response of impacted plate is shown in Figure 2.7 where local indentation $\alpha$ in Figure 2.7(a) is a typical characteristics 


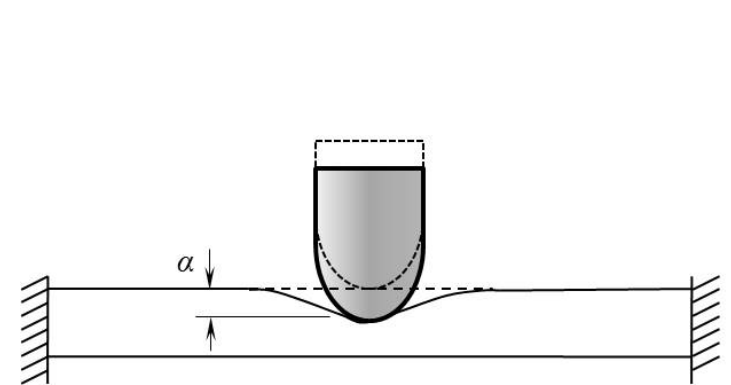

(a)

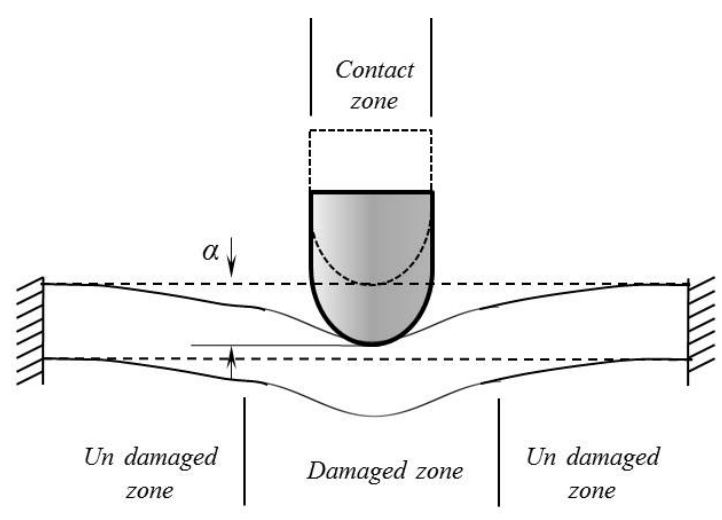

(b)

Figure 2.7: Plate response under impact loading; (a) pure local deflection (b) Combined local and global deflection

of small-mass, high velocity impact on thick plate and combined local and global deflection in Figure 2.7(b) is for large-mass and low velocity impact on thin plate.

The local deflection of a plate is described by means of proper Hertz contact law, which relates local indentation $\alpha$ and contact force $F$ as;

$$
F=k_{\alpha} \alpha^{n}
$$

where $k_{\alpha}$ is the contact stiffness between impactor and plate, $n$ is the power parameter and $\alpha$ is the local indentation.

In general, the shape function of plate flexure seems to be a key parameter to determine the accuracy of any analytical model. It is feasible only when assumed function tends to be admissible and reflect appropriate deflection behaviour of the concerned plate structure. Depending on the width-to-thickness ratio of the plate, the global deflection of a plate is dominated either by bending, membrane stretching, bending-membrane combination or transverse shear [66]. For thin plates, transverse shear deformation is very small and often neglected. It is difficult to introduce different profile shapes for damaged and undamaged zone and often no difference had been made between them. Different forms of deflection profile shapes have been suggested to model clamped circular plate under indentation load as listed in Table 
Table 2.3: Clamped circular flexural profile shapes for large deflection analysis of a plate

\begin{tabular}{|c|c|c|c|}
\hline \multicolumn{2}{|l|}{ Reference } & \multirow{2}{*}{$\begin{array}{l}\text { Deflection profile } \\
w=C_{1}\left(1-\frac{r^{2}}{a^{2}}+2 \frac{r^{2}}{a^{2}} \ln \left(\frac{r}{a}\right)\right)\end{array}$} & \multirow{2}{*}{$\begin{array}{l}\text { Status } \\
\text { Admissible }\end{array}$} \\
\hline Shivakumar et.al 67 & & & \\
\hline Timoshenko 66 & & $w=C_{1}\left(1-\frac{r^{2}}{a^{2}}\right)^{2}$ & Admissible \\
\hline Hashem Babaei et.al & 68 & $w=C_{1}\left(\operatorname{Bessel} J\left(0, \frac{c r}{a}\right)\right)$ & $\begin{array}{l}\text { Not Admissible (finite slope at } \\
\text { center) }\end{array}$ \\
\hline Tsamasphyros et.al 6 & 69 & $w=C_{1}\left(1-\sin \frac{\pi r}{2 a}\right)$ & $\begin{array}{l}\text { Not Admissible (finite slope at } \\
\text { clamped end) }\end{array}$ \\
\hline
\end{tabular}

For low velocity impact, the strain rate and wave propagation effects are negligible and the static properties of the materials are used without any alteration. According to Abrate [70], the available solution for impact models of laminated materials are classified into four categories; spring-mass (SM) approach [71,72], energy (E) approach models 67,73,74, complete models [75 based on Classical Plate theory (CPT) and Mindlin's First order Shear Deformation Theory (FSDT) [76] and combinations of above as listed in Table 2.4

Table 2.4: Analytical solution methods for FMLs

\begin{tabular}{|c|c|c|c|}
\hline Reference & Solution Method & Test condition & Metal behaviour \\
\hline Vlot 18 & $\mathrm{E}+\mathrm{SM}$ & LVI & Elastic-Plastic \\
\hline Hoo Fatt et.al 77 & $\mathrm{E}+\mathrm{SM}$ & QSI + Ballistic & Rigid Plastic \\
\hline Tsamasphyros et.al 69.78 & $\mathrm{E}+\mathrm{SM}$ & QSI+LVI & Rigid Plastic \\
\hline $\begin{array}{ll}\text { Payeganeh et.al } 79 \\
\end{array}$ & $\mathrm{FSDT}+\mathrm{SM}$ & LVI & ELastic \\
\hline Moriniere et.al 54,80 & $\mathrm{CLT}+\mathrm{E}$ & LVI & Elastic-Plastic \\
\hline
\end{tabular}

\subsubsection{Analytical developments in FML}

Based on the spring-mass system [81], Vlot [13 proposed the first analytical model for FML to determine closed form solutions for plate deflection, contact force, contact time. For the impactor of mass $m_{p}$ with initial velocity $v_{0}$ impacted on 

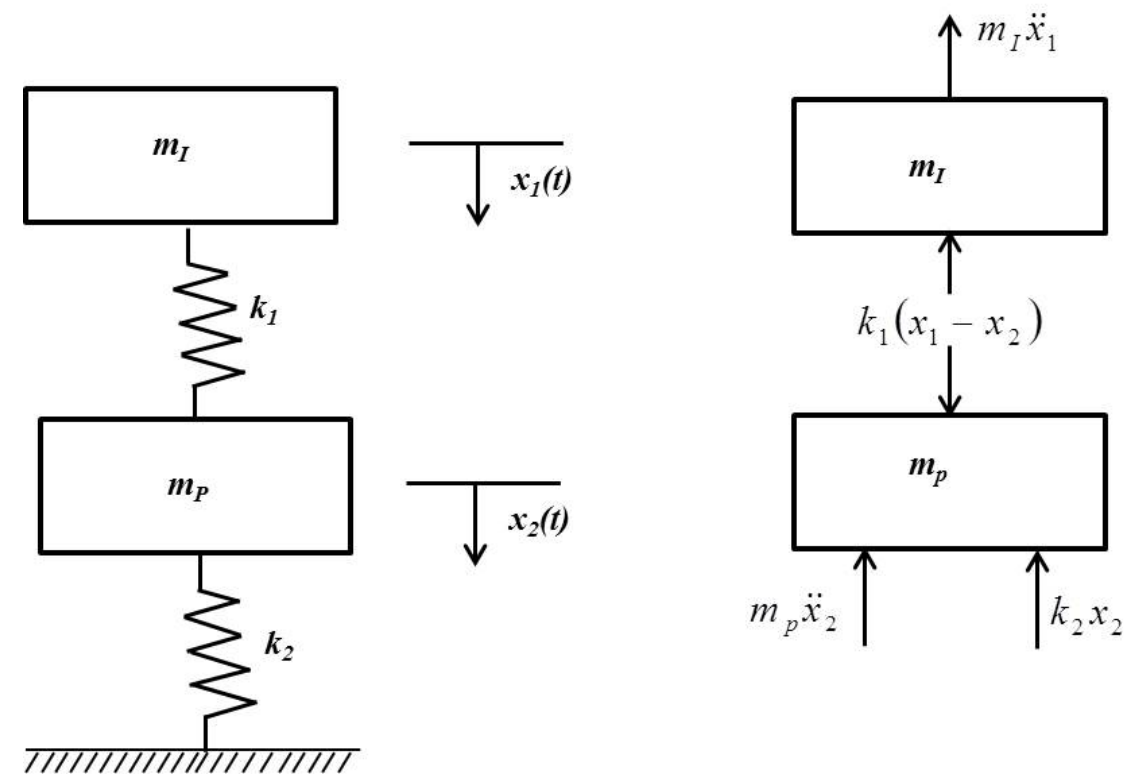

Figure 2.8: 2-DOF spring mass system for modeling low velocity impact phenomenon circular specimen of equivalent mass $m_{e q}$ having stiffness $C$, the solution for impact response is obtained by solving simple governing differential equation of form:

$$
m_{\text {tot }} \ddot{w}_{0}+C w_{0}=0
$$

where, $m_{t o t}=m_{e q}+m_{p}$ and $w_{0}$ is the centre deflection of the plate.

With the help of Von karman strain displacement relations [67], modified Hertzian contact law 82, and Newmark numerical algorithm [83, Vlot [18] developed an efficient full scale non-linear elastic model for impact on rectangular FML plate.

Payeganeh et al. [79 assumes a 2-DOF (degrees of freedom) spring mass system(as shown in Figure 2.8) to determine contact force. In the figure, symbols $m_{I}$, $m_{p}, x_{1}$ and $x_{2}$ represents mass, displacement of impactor and FML plate respectively and $k_{1}$ and $k_{2}$ represents contact and bending-shear stiffness respectively.

Using Choi's linearized contact law [84], the governing equations of above 2-DOF spring mass system is expressed in the form:

$$
\begin{gathered}
m_{1} \ddot{x}_{1}+k_{1}\left(x_{1}-x_{2}\right)=0 \\
m_{p} \ddot{x_{2}}+k_{2} x_{2}-k_{1}\left(x_{1}-x_{2}\right)=0
\end{gathered}
$$


With the initial conditions of $x_{1}(0)=x_{2}(0)=0, \dot{x_{1}}(0)=\mathrm{V}$ and $\dot{x_{2}}(0)=0$ at time $t=0$.

The obtained contact force history was substituted to Mindlin's first order shear deformation theory. The complete equation of motion was formulated in terms of Navier's approach having all unknown variables (deflection, in-plane shear deformations) in terms of Fourier series. The final equations were solved numerically with the help of mathematical solver MATLAB, and all the structural characteristics like deflection under the impactor, strain, stress informations were found. However, neglecting the membrane effect, rotary inertia, plasticity behaviour of layers, implementing linearized contact law and excluding the damage effects are some of the major limitations.

Tsamasphyros et.al [69] used the total potential energy approach to derive loaddeflection relationship of a circular GLARE plate of radius a under static load using Rayleigh Ritz method. The material behavior of aluminum and GFRP layer was assumed as rigid-perfectly plastic and linear elastic respectively and corresponding strain energy equations are formulated. Assuming very large deflection, the resistance of the plate to bending was assumed negligible (all $D_{i j}$ terms) and only the effect of membrane resistance (all $A_{i j}$ terms) was taken in to account [15]. Similar to the expression used by Hoo fatt et.al [77], the derived load-deflection relation takes the form,

$$
\begin{gathered}
P=k_{p} w_{0}+k_{e l} w_{0}^{3} \\
k_{p}=C_{1} N_{x}+C_{2} N_{y}+C_{3} N_{x y} \\
k_{e l}=\frac{1}{a^{2}}\left[C_{4} A_{11}+C_{5} A_{22}+C_{6}\left(A_{12}+A_{66}\right)\right]
\end{gathered}
$$

where $k_{p}, k_{e l}$ are the stiffness due to plastic and membrane effects respectively and $C_{i}$ are the constant coefficients.

On substituting the contact force relation given in equation (2.6) to a spring mass system having 1-DOF degree of freedom as shown in Figure 2.9, the dynamic response of the plate can be formulated [78;

In Figure 2.9, the symbols $M_{0}$ and $m_{e}$ are the mass of impactor and effective 

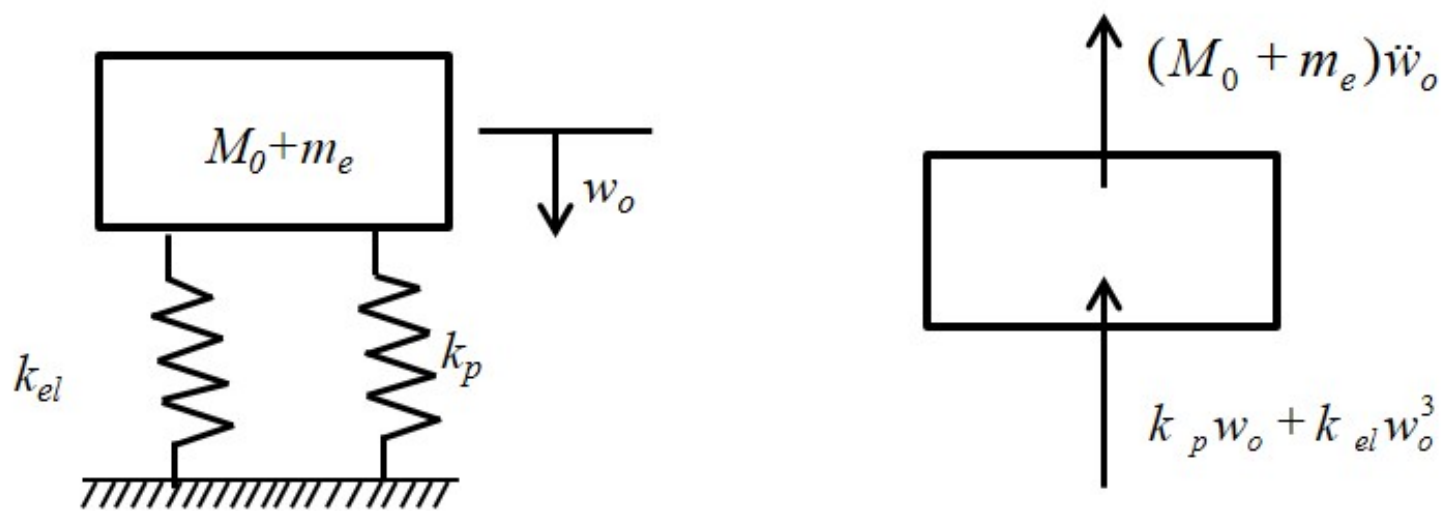

Figure 2.9: 1-DOF spring mass system for modeling low velocity impact phenomenon plate mass respectively. The total response of the problem is usually divided in to three stages with different initial conditions as shown in Table 2.5.

Table 2.5: Governing Differential Equations of Motion and initial conditions

\begin{tabular}{llll}
\hline \multirow{2}{*}{ Stage } & Governing differential equation & \multicolumn{2}{c}{ Initial Conditions } \\
\cline { 3 - 4 } & & Displacement & Velocity \\
\hline $\begin{array}{l}\text { Loading stage } \\
\text { before delami- }\end{array}$ & $\left(M_{0}+m_{e}\right) \ddot{w}_{0}+k_{p} w_{0}+k_{e l} w_{0}^{3}=0$ & $w_{0}(0)=0$ & $\dot{w}_{0}(0)=v$ \\
nation & & $w_{0}(0)=w_{0}^{d}$ & \\
\hline $\begin{array}{l}\text { Loading stage } \\
\text { after delami- }\end{array}$ & $\left(M_{0}+m_{e}\right) \ddot{w}_{0}+k_{p} w_{0}+k_{e l} w_{0}^{3}=0$ & \\
nation & & & \\
\hline $\begin{array}{l}\text { Unloading } \\
\text { stage }\end{array}$ & $\left(M_{0}+m_{e}\right) \ddot{w}_{0}+2 k_{p} w_{0}+k_{e l} w_{0}^{3}-k_{p} w_{0}^{\text {max }}=0$ & $w_{0}(0)=w_{0}^{\text {max }}$ & $\dot{w}_{0}(0)=0$ \\
\hline \hline
\end{tabular}

By solving the three initial value problems listed in Table 2.5, the author can predict histories of contact load, displacement, velocity, kinetic energy [85].

Recently, Moriniere [54,80] developed a comprehensive quasi-static analytical model to study the low-velocity impact response of rectangular clamped GLARE FML plates loaded with a rigid hemispherical indenter. The contribution of metal, composite layer and its interaction on impact response are taken in to account through the modified deflection function of form given by Lin [86] narrated in equation (2.9). On combining FSDT, CLT, energy conservation principle and newton's law of motion, a closed form solution is achieved in terms of force, velocity, displacement and energy absorbed. 


$$
w(x, y)= \begin{cases}w_{\text {max }}, & 0 \leq x \leq R_{a}, 0 \leq y \leq R_{b} \\ M V F w_{0 A L}\left(1-\frac{x}{\alpha a}\right)^{m}\left(1-\frac{y}{\beta b}\right)^{m} & \\ +(1-M V F) w_{0 G E}\left(1-\left(\frac{x}{\alpha a}\right)^{n}\right) & R_{a} \leq x \leq \frac{a}{2}, R_{b} \leq y \leq \frac{b}{2}, \\ \left(1-\left(\frac{y}{\beta b}\right)^{n}\right), & \end{cases}
$$

where $w_{0 A L}=\frac{w_{\max }}{\left(1-\frac{R_{a}}{\alpha a}\right)^{m}\left(1-\frac{R_{b}}{\beta b}\right)^{m}}$,

$w_{0 G E}=\frac{w_{\max }}{\left(1-\left(\frac{R_{a}}{\alpha a}\right)^{n}\right)\left(1-\left(\frac{R_{b}}{\beta b}\right)^{n}\right)}$ with $a \geq b ; 0 \leq \alpha \leq 1$ and $0 \leq \beta \leq 1$

Integration of strain rate effects, inclusion of metal plasticity, and consideration of both intraply and interplay damage scenarios are some of the important features of the developed model.

\subsection{Conclusion}

This chapter reviewed existing research progress devoted to quasi-static indentation and low velocity impact response of FMLs. An overview of the experimental investigations on FMLs are listed in terms of their main influencing factors. Subsequently, developments achieved in the numerical modeling research stream to simulate the whole impact behaviour scenario are collected and reviewed. From the current numerical models, it is recommended to use Abaqus/Explicit FE platform to model the dynamic response of FMLs to achieve utmost computational efficiency. A summary of analytical modelling is also carried out and addressed the complex non-linear formulations involved.

Some of the issues that have been noticed through this comprehensive review are;

- Even though woven fabric composites showed better damage tolerance feature [7, 10, 87, its role in FML is not yet well understood. Hence it is necessary 
to understand the individual and fundamental role of constituent materials to impact resistance and energy absorption.

- Despite FML shows enhanced indentation and impact resistance than their corresponding monolithic constituents, the contribution of each individual material to the resultant response is not well understood.

- Debonding and delamination are the important damage modes of indentation and low velocity impact load [55]. With the absence of fibre bridging phenomenon in woven composites, debonding might severely affect the integrity of woven FML. Thus a thorough understanding of the interface behaviour and durability of woven FML is essential. The thin interfacial zones between plies is the main problem area as it is difficult to accurately estimate de-bond boundary via. cross sectioning and NDT technique. There is no rigorous numerical work currently available to model those failure phenomena. The following chapters will address the aforementioned issues. 


\section{Chapter 3}

\section{Test configurations and constitutive material models}

This chapter broadly discusses the experimental and numerical methods used to predict the indentation and impact behaviour. Details of experimental set up and mathematical formulation present behind the screen are described in Section 3.1 . Framework and pre-processing definitions used in the FE model are discussed in Section 3.2. Section 3.3 - 3.5 presents the details of constitutive relations of each material used in the FE model to define the undamaged and damaged behaviour. Reliability of proposed constitutive model is also examined by comparing numerical results of indentation and impact behaviour of each material with experimental results in respective sections.

\subsection{Experimental configurations}

\subsubsection{Quasi-Static Indentation (QSI) test}

Prior to low velocity impact (LVI) test, QSI test provides extensive beneficial information about material behaviour of hybrid sample. Through QSI test, damage initiation, propagation of each failure mode can be easily tracked. Moreover, panel deflection and transverse force measurements are easily available and corresponding energy range required to produce respective failure mode is possible [17]. QSI tests are conducted based on the standard described in ASTM D6264 88 using In- 


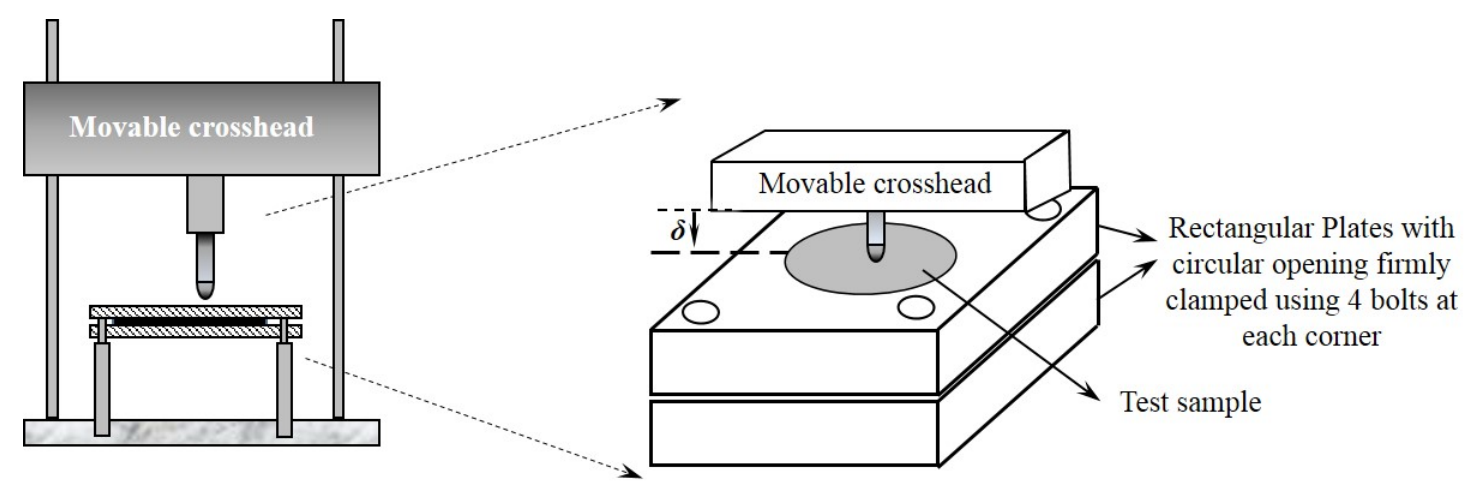

Figure 3.1: Typical Quasi-static indentation test apparatus

stron testing machine 5500R at displacement rate of $1 \mathrm{~mm} / \mathrm{min}$. Panels are clamped between the steel plates having a central circular opening of $76.4 \mathrm{~mm}$ and firmly tightened using 4 bolts. Typical apparatus mounted with hemispherical indenter used for QSI test along with clamping fixture geometry are shown in Figure 3.1 The resistance force to indentation $(\mathrm{P})$ of sample was recorded in terms of indenter displacement $(\delta)$ using computerized data acquisition system. From the P- $\delta$ curve, information regarding internal energy absorbed in total and for each failure stages are evaluated through piece wise integration. Three samples were tested for each case and two of them were loaded until the contact load drops by $90 \%$ of respective peak value and one was stopped intermittently after the load-displacement $(\mathrm{P}-\delta)$ curve shows initial drop. After visual inspection, the samples were mechanically bisected in order to evaluate the loci of sub-surface damage and MCI de-bonding boundary.

\subsubsection{Low velocity impact (LVI) test}

Several experimental techniques have been used to study the LVI performance of flat target specimen. Izod, Charpy and Instrumented drop weight impact tester are some of the well-known methods. Instrumented drop weight tester is the standard representative used to study the LVI performance of composite and other hybrid samples. Tests are carried out based on the guidelines described in ASTM D5420 89 using Instron Dynatup 8250 depicted in Figure 3.2. Force transducer is mounted inside the impactor tup which collects the information of contact force history and 

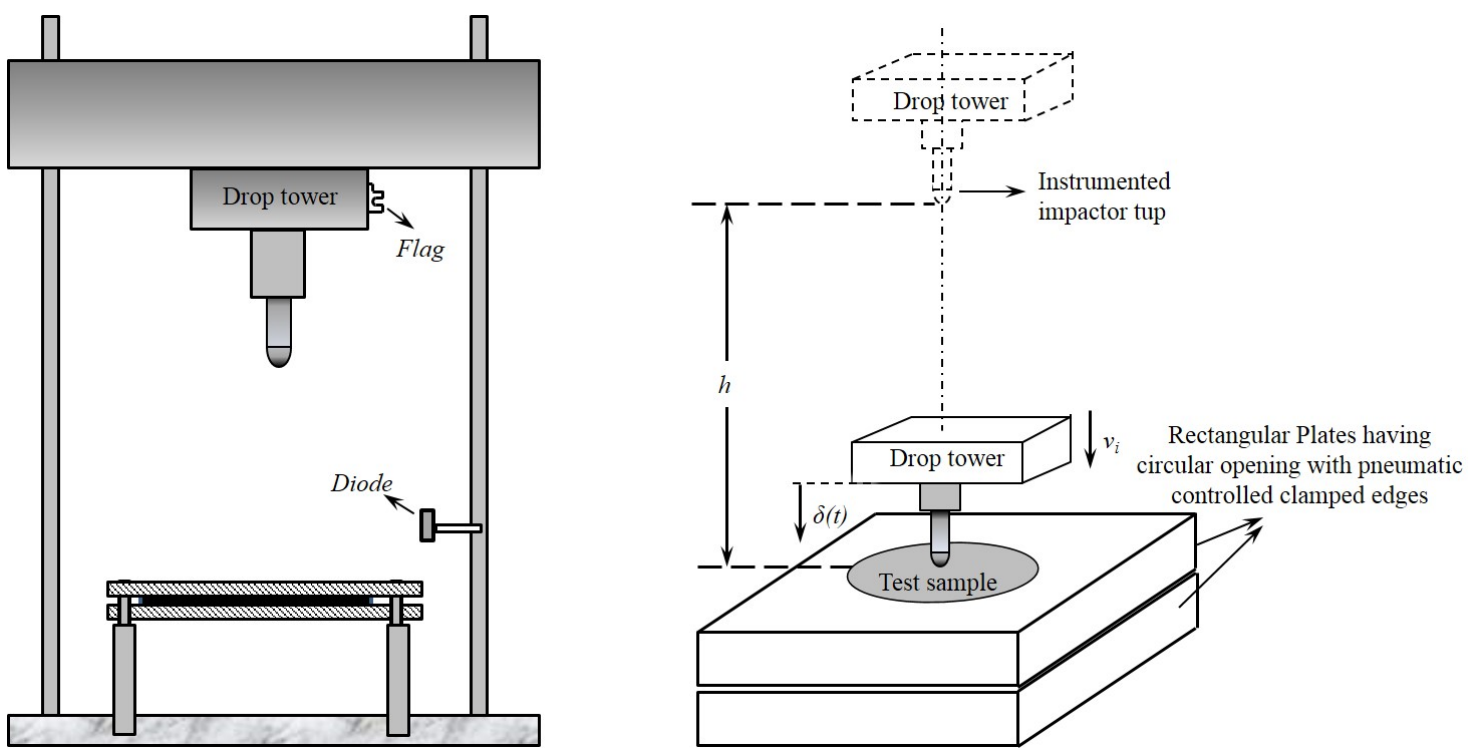

Figure 3.2: Typical Low velocity drop weight impact test apparatus

using photo diode emitter-detector and double pronged flag assembly, the magnitude of velocity just before the moment of impact $\left(V_{m e s}\right)$ is measured and then converted to velocity $\left(V_{i}\right)$ at the moment of impact $\left(t_{i}\right)$ based on following equation of motion;

$$
\begin{aligned}
& V_{\text {mes }}=\frac{w_{\text {flag }}}{t_{\text {flag }}}+\frac{1}{2} g t_{\text {flag }} \\
& V_{i}=V_{\text {mes }}+g\left(t_{1}-t_{2}\right)
\end{aligned}
$$

where, $w_{\text {flag }}=\left(x_{2}-x_{1}\right)$ is the effective flag width and $t_{\text {flag }}=\left(t_{2}-t_{1}\right)$ is the time duration across the flanges to cross the velocity detector and $g$ is the acceleration due to gravity $\left(9.81 \mathrm{~ms}^{-2}\right)$. In the case of free fall weight similar to the present study, $V_{i}$ can be verified by equating potential energy and kinetic energy of the system;

$$
V_{i}=\sqrt{2 g h}
$$

where, $h$ is the vertical drop height. Force history is measured from the onset of contact between impactor and sample until the impactor rebound/completely perforated from/through the sample. The contact force $F(t)$ is assumed to be sum of the resistive force of target sample measured by force transducer and gravitational 
force due to weight of drop tower. Mathematically,

$$
F(t)=m g-p(t)
$$

where, $m$ is the mass of drop tower and $p(t)$ is the resistive force measured by force transducer at time $t$. Energy history can be obtained by integrating the contact force signal $F(t)$ as follows, From equation (3.4),

$$
\begin{gathered}
a(t)=\frac{F_{t}}{m} \\
v(t)=v_{i}+g t-\int_{0}^{t} \frac{p(\tau)}{m} d \tau \\
x(t)=x_{0}+v_{i} t+g \frac{t^{2}}{2}-\int_{0}^{t}\left(\int_{0}^{t} \frac{p(\tau)}{m} d \tau\right) d \tau
\end{gathered}
$$

where, $a(t), v(t)$ and $x(t)$ are acceleration, velocity and position of drop tower at time $t$ respectively and $x_{0}$ is the position of impactor at time $t=0$. Based on the conservation of energy principle, the total energy of drop tower $E(t)$ at any time $t$ is given by;

$$
E(t)=K(t)+V(t)+E_{a}(t)=\text { constant }
$$

In equation (3.8), $K(t)=\frac{1}{2} m v^{2}(t)$ and $V(t)=-m g x(t)$ represent kinetic and potential energy of drop tower at time $t$ respectively and $E_{a}(t)$ defines the amount of energy absorbed by the sample at time $t$. At the onset of impact $(t=0), V(0)=0$, $E_{a}(0)=0$, equation 3.8$)$ becomes

$$
E(0)=K(0)=\text { constant }
$$

On interpreting condition (3.9) in to equation (3.8), the expression for $E_{a}(t)$ is given by,

$$
E_{a}(t)=K(0)-K(t)-V(t)
$$


or

$$
E_{a}(t)=\frac{1}{2} m v^{2}(0)-\frac{1}{2} m v^{2}(t)+m g x(t)
$$

where $v(t)$ and $x(t)$ are obtained from equation (3.6) and 3.7) respectively. Utilizing equation (3.2), (3.4), 3.11) the quantity of impact velocity, contact force history and energy absorbed values are readily obtained. Specifications of target and impactor used in this thesis are listed in Table 3.1.

Table 3.1: Specifications of target sample and impactor utilized in the experimental study

\begin{tabular}{lll}
\hline Component & Parameter & Specification/Nature \\
\hline \multirow{3}{*}{ Target sample } & Shape & Flat and Square \\
& In-plane size & 100mm X 100mm \\
& Thickness & Variable \\
& Boundary condition & Clamped \\
\hline & Shape & Hemispherical \\
& Radius & $6.55 \mathrm{~mm}$ \\
Impactor* & Material & Steel \\
& Mass & Variable \\
& Impact angle & Normal to the target sam- \\
& & ple \\
\hline
\end{tabular}

\footnotetext{
* The indenter used in QSI has identical geometrical shape and dimension as LVI
}

\subsection{Details of FE model}

All the pre-processing, solving and post-processing numerical strategies of indentation and impact simulations are performed in dynamic explicit FE platform in ABAQUS code. In order to attenuate the dynamic effects and for truly simulating the indentation process as quasi-static, the total time period in dynamic explicit environment is considered 10 times to that of time period required to produce lowest natural frequency in standard environment. Behaviour of metal layer is defined as elastic, isotropic hardening based plastic behaviour with damage initiation and evolution phenomenon based on ductile damage and fracture energy criterion respectively. The layer is discretized with 3-D reduced integrated solid element (C3D8R) with 4 elements along thickness direction to avoid hourglass and shear locking phenomenon. From experimental observations, no sign of interlaminar delamination exists between the woven FRP layers. Thus, the inter-laminar region between FRP 


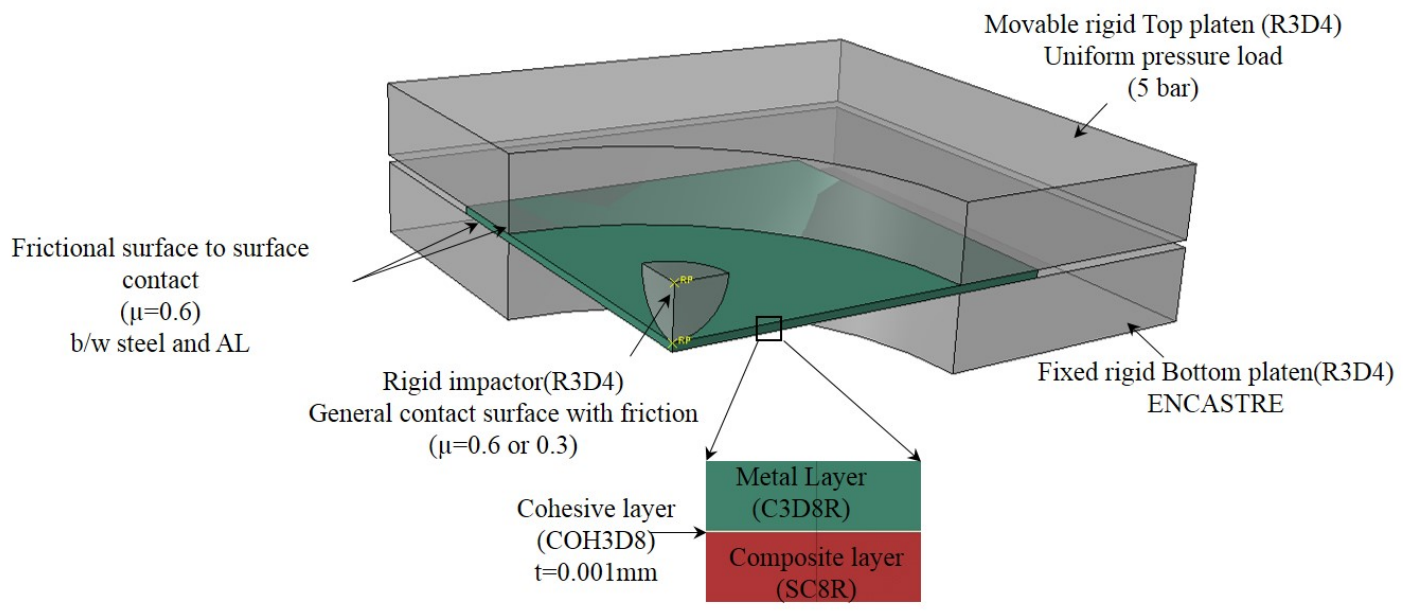

Figure 3.3: Numerical model setup for QSI and LVI FE simulation

lamina is assumed perfect and FRP laminate of given thickness is modelled with single continuum shell SC8R element. The behaviour of FRP layer is based on the constitutive model proposed by Johnson [90] which include initial orthotropic elastic behaviour and continuum damage model for the prediction of damage initiation and damage evolution. The stated model is incorporated in to ABAQUS FE code via inbuilt VUMAT subroutine ABQ_PLY_FABRIC.

It is required to have an interface element for a resin rich region in order to predict interlayer delamination and successive degradation [91,92]. To comply with debonding scenes observed between metal and FRP layers known as Metal-Composite Interface (MCI), those regions in equivalent FE model are discretized using three dimensional cohesive interface elements (COH3D8) to virtually simulate de-bonded interfaces. The material model of cohesive element follows uncoupled, bi-linear traction-separation constitutive behaviour with quadratic stress based damage initiation and mixed mode energy based damage evolution definition. The adhesive layer between metal and composite layer of FML panel is generally very thin and almost constant in thickness. The interface cohesive element of thickness $10^{-6} \mathrm{~m}$ is introduced and define the interaction with adjacent layers through regular shared mesh pattern.

Figure 3.3 illustrates the geometry and boundary conditions employed in the FE model (only quarter model is shown for better visualization of all boundary 
conditions). FML test sample of size $100 \mathrm{~mm}$ X $100 \mathrm{~mm}$ is assembled between the rigid platens having a circular opening of radius $38.4 \mathrm{~mm}$. The rigid platens replicate the clamped fixture set up as used in experiments where top plate is applied with 5 bar distributed pressure load to replicate pneumatic pressure and the bottom plate is held fixed. The downward movement of $6.55 \mathrm{~mm}$ radius impactor is governed through a single reference point (RP) having free boundary condition to move along out of plane direction and pre-defined with initial loading condition; displacement for QSI and incident impact velocity for LVI. Both fixture plates and impactor geometry is chosen to model as rigid body using R3D4 elements.

Two different kinds of contact conditions were used to define the interaction of confronting surfaces. One was between the sample and rigid plates associated by surface-surface contact algorithm and the other between impactor and sample with general contact algorithm. In addition, frictional effects are included based on coulomb friction model with frictional coefficient of $\mu=0.6$ for former and $\mu=0.6$ (or 0.3) for latter governing static QSI (or dynamic LVI) test. The stated numbers are the typical values for contact between steel and aluminium surfaces.

Historical output data such as total contact force (normal contact force + tangential frictional force), impactor displacement and internal energy absorbed by the target are extracted and compared with obtained experimental results. Numerically, the absorbed internal energy $\left(E_{I}\right)$ of dynamic system has been split into different sub-energy components and can be written as:

$$
E_{I}=E_{E L}+E_{P L}+E_{D M D}+E_{C D}+E_{A}+E_{D C}+E_{F C}
$$

where the nomenclature of above notations takes the following definition in sequence; Internal energy is equal to the sum of recoverable elastic strain energy, the energy dissipated through plasticity, energy dissipated through damage, energy dissipated through visco-elasticity or creep, artificial strain energy, energy dissipated through distortion control and finally fluid cavity energy. With appropriate input parameters, the above components can be quantified for any given loading case. Only 
the first three components are the subject of interest in the present work, because those phenomenon are significant in energy absorbing mechanism of FML panel. Moreover, these quantifications are helpful in estimating the role of each material constituent at any instant of given load step. Besides, simulated damage shapes of each material layer, de-bonding boundary of MCI and cross sectional damage morphology are correlated with experimental damage surface. Details of the constitutive material models used in the FE simulations are described in following sections.

\subsection{Material model for the metal layer}

\subsubsection{Details of constitutive model}

The constitutive law used to define metal layer behaviour is shown in Figure 3.4. Metal layer behaves as elastic-plastic material with rate independent behaviour (due to quasi-static assumption). The initial linear elastic response of material based on generalized Hookes law is defined as;

$$
\sigma_{i j}=C_{i j k l} \varepsilon_{i j}^{e}
$$

where $\varepsilon_{i j}^{e}$ is the elastic strain and $C_{i j k l}$ is the fourth order elasticity tensor.

The irreversible deformation of metal layer follows Von Mises plastic model with isotropic hardening plasticity defined in terms of equivalent or effective stress $\left(\sigma_{e q}\right)$ and equivalent plastic strain $\left(\varepsilon_{e q}^{p l}\right)$ in tabular form related by the equation;

$$
f\left(\sigma_{i j}, \varepsilon_{e q}^{p l}\right)=\sigma_{e q}-Y\left(\varepsilon_{e q}^{p l}\right) \quad \forall \quad f\left(\sigma_{i j}\right)= \begin{cases}<0, & \text { Elastic } \\ =0, & \text { Plastic }\end{cases}
$$

where $\sigma_{e q}=\sqrt{\frac{3}{2} S_{i j} S_{i j}}$ and $S_{i j}=\sigma_{i j}-\frac{1}{3} \sigma_{k k} \delta_{i j}$ are the equivalent or effective stress and deviatoric stress tensor respectively. Damage onset is formulated using JohnsonCook dynamic failure model $\left[93\right.$ which equates equivalent plastic strain $\left(\bar{\varepsilon}_{0}^{p}\right)$ at 


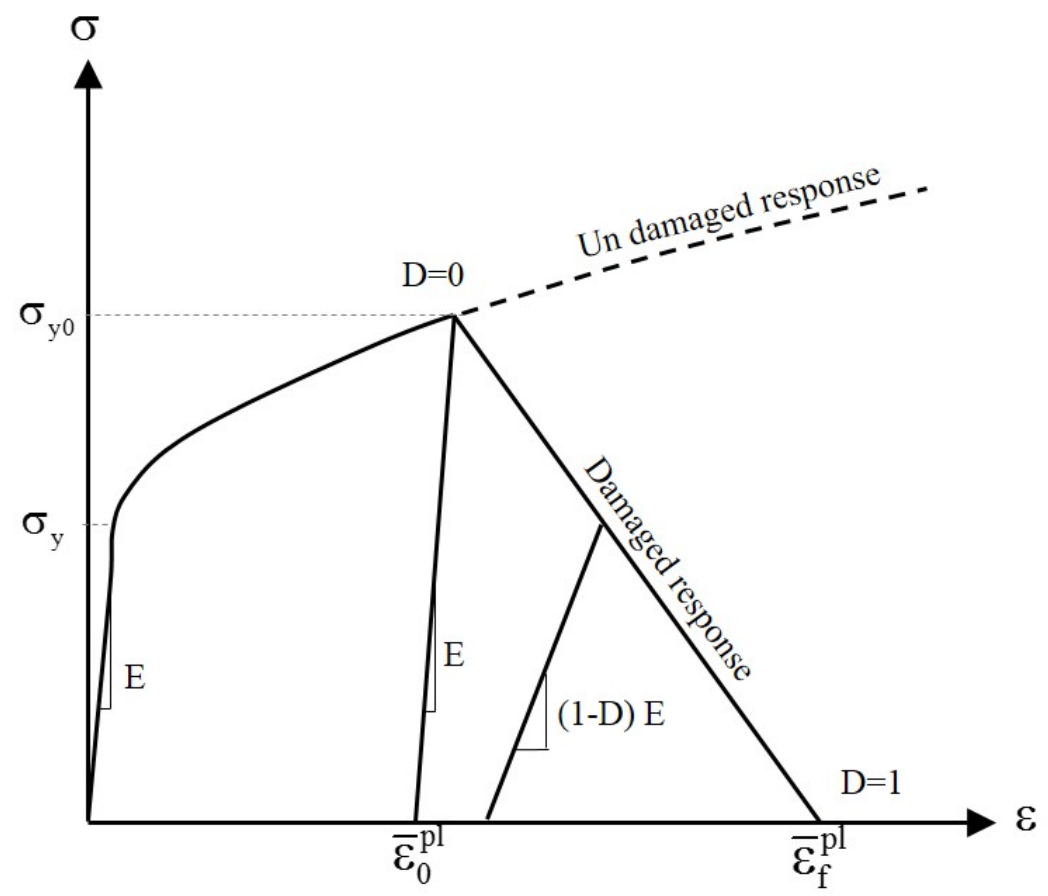

Figure 3.4: Typical constitutive response of metal layer

element integration with series of damage parameters through the relation of form;

$$
\bar{\varepsilon}_{0}^{p}=\left[d_{1}+d_{2} \exp \left(-d_{3} \frac{p}{q}\right)\right]\left[1+d_{4} \ln \frac{\dot{\bar{\varepsilon}}^{p}}{\dot{\varepsilon}_{0}}\right]
$$

where, $p$ is the hydrostatic stress, $q$ is the Von Mises stress, $d_{1}-d_{4}$ are failure parameters and $\dot{\varepsilon}_{0}$ is the reference strain rate $=1 s^{-1}$. Failure is assumed to occur when the scalar variable $\omega$ defined in equation (3.16) approaches unity.

$$
\omega=\sum\left(\frac{\Delta \bar{\varepsilon}^{p}}{\bar{\varepsilon}_{0}^{p}}\right)
$$

where $\Delta \bar{\varepsilon}^{p}$ is the equivalent plastic strain increment between successive increments.

The softening response after the damage initiation is characterized by establishing damage evolution criterion expressed in terms of fracture energy $\left(G_{f}\right)$ expressed in the form:

$$
G_{f}=\int_{\bar{\varepsilon}_{0}^{p}}^{\bar{\varepsilon}_{f}^{p}} L \sigma_{y} d \bar{\varepsilon}^{p}
$$

where, $\bar{\varepsilon}_{f}^{p}$ is equivalent plastic strain at failure, $\mathrm{L}$ is the characteristic length of the element which is generally required to alleviate mesh dependency on damage 


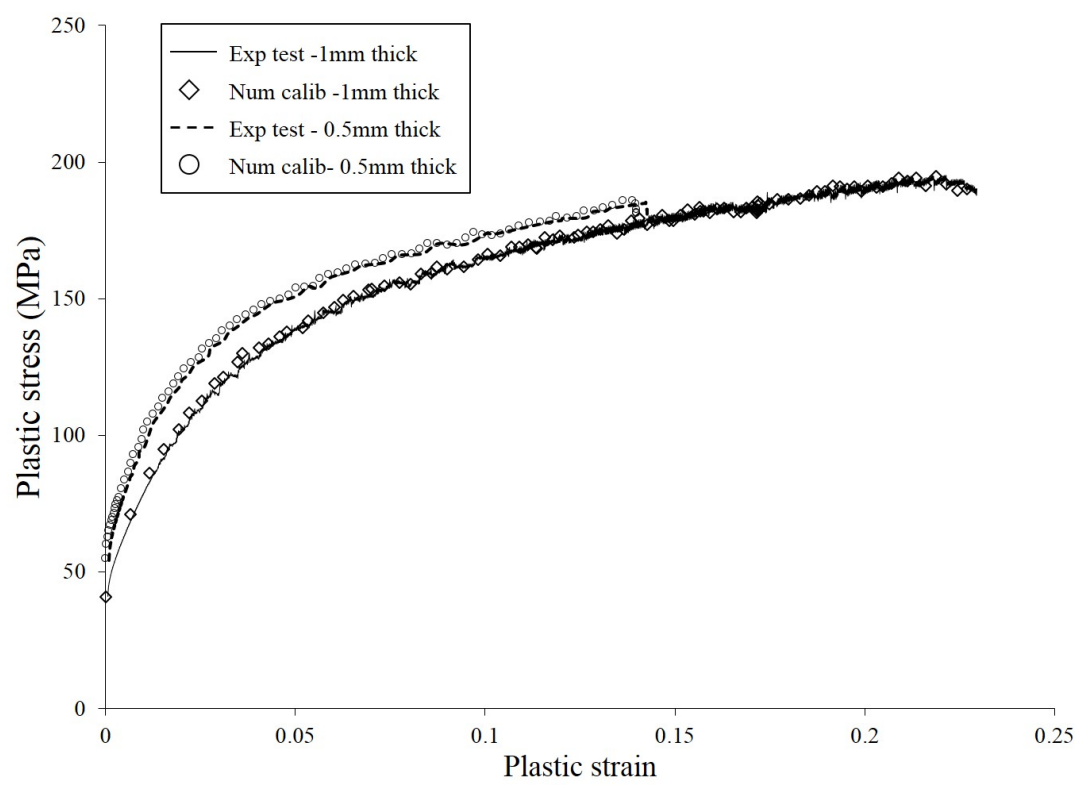

Figure 3.5: Plastic stress-strain curve of different thick aluminium 2024-O sheet response $[94$.

\subsubsection{Calibration of material parameters}

In the present work, the metal layer of the FML panel used is soft, annealed Aluminium alloy $2024-\mathrm{O}$ of thickness $0.5 \mathrm{~mm}$ and $1 \mathrm{~mm}$. The material parameters required to simulate the elastic and isotropic hardening plastic response can be found using simple tensile test according to ASTM standard [95]. Significant influence of sheet thickness on tensile strength and ductility of soft AL alloys has been reported by various researchers 96,97 . Thus, tensile test were performed for each sheet thickness. Similar to the observations noticed by Suh et.al [96], the strain to failure $\varepsilon$ was found to decrease with decrease in metal sheet thickness. Predicted true plastic stress versus true plastic strain values along with calibrated values to define isotropic hardening behaviour of metal layer in numerical model is shown in Figure 3.5 Despite the absence of the Johnson cook failure parameters $\left(d_{1}-d_{4}\right)$ for AL 2024-O alloy, parameters of 2024-T3 alloy has been substituted to predict damage onset of 2024-O alloy. Such substitution is applicable for the present situation since failure initiation by Johnson cook model is merely governed by equivalent plastic strain $\bar{\varepsilon}^{p}$ which is almost same for both alloys and does not greatly affect the resultant failure initiation response [98]. 
Typical fracture energy value of aluminium alloy is $20 \mathrm{~N} / \mathrm{mm}$. However, with the interference of mesh dependent parameter namely characteristic element length (L), and the in-built assumption of constant yield stress after damage initiation in ABAQUS code, the fracture energy magnitude is not any more material property and it depends on assumed damage onset stress and element type used to discretize the layer. Conservatively, a value of $7.5 \mathrm{~N} / \mathrm{mm}$ and $10 \mathrm{~N} / \mathrm{mm}$ is assumed to be a fracture energy value for $0.5 \mathrm{~mm}$ and $1 \mathrm{~mm}$ thick AL layers respectively according to equation (3.17), based on the assumed damage onset stress of $150 \mathrm{MPa}$ and corresponding plastic strain values given by Figure 3.5. The material properties defined to the metal layer elements are listed in Table 3.2 .

Table 3.2: Material properties of AL-2024-O metal sheet

\begin{tabular}{ll}
\hline Response & \multicolumn{1}{c}{ Property } \\
\hline Elastic & Young's Modulus E=70 GPa \\
Plastic & Data points retrieved from Figure 3.5 \\
Damage Initiation* & $d_{1}=d_{2}=0.13, d_{3}=1.5, d_{4}=0.011$ \\
Damage Evolution ${ }^{\ddagger}$ & Fracture energy $G_{f}=10(7.5) \mathrm{N} / \mathrm{mm}$ \\
\hline${ }^{*}$ From ref 98. & \\
${ }^{\ddagger}$ Values inside the parentheses is for aluminium sheet of $0.5 \mathrm{~mm}$ thickness.
\end{tabular}

\subsubsection{Finite element analysis}

The suitability of the constitutive model to simulate indentation and impact response of bare aluminium plates is studied in this section. The numerical predicted results are validated with experimental observations both in terms of characteristic curves and failure modes.

\subsubsection{Indentation response of bare aluminium plate}

Indentation response of aluminium plate of $0.5 \mathrm{~mm}$ and $1 \mathrm{~mm}$ thickness are shown in Figure 3.6(a). Initial elastic-plastic response and peak load (also equal to threshold load to initiate softening) predicted by the FE simulations are in good agreement with those of experimental measurements. Failure prediction realized through drop in contact load confirm that equivalent plastic strain $\left(\bar{\varepsilon}_{p}^{0}\right)$ based Johnson cook failure criterion is well capable of predicting the damage onset. 


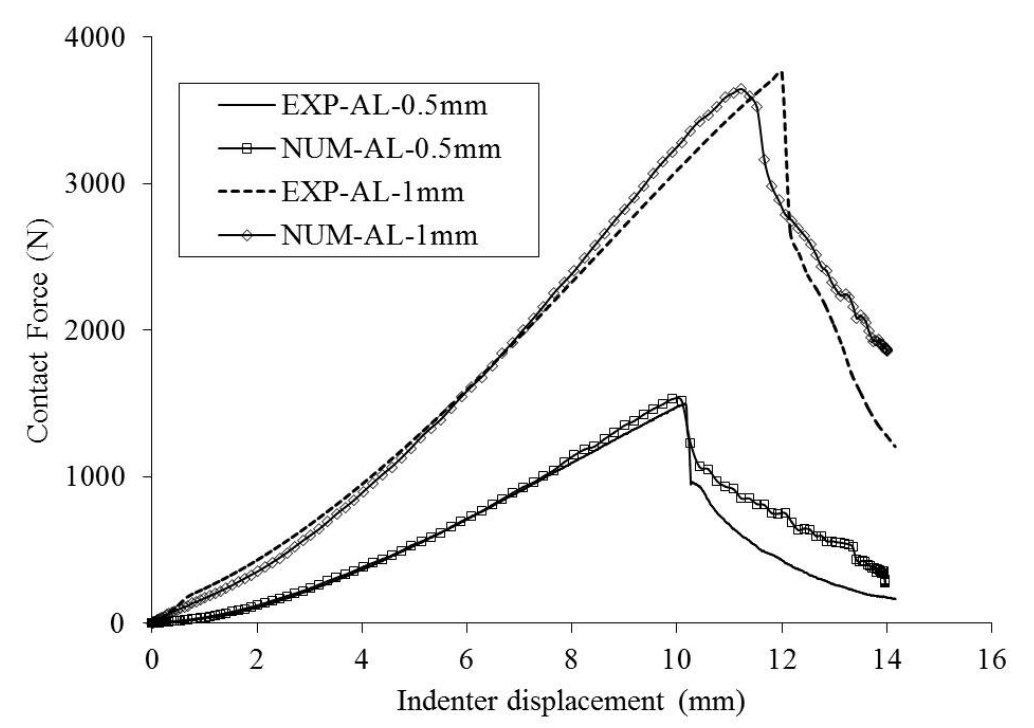

(a)
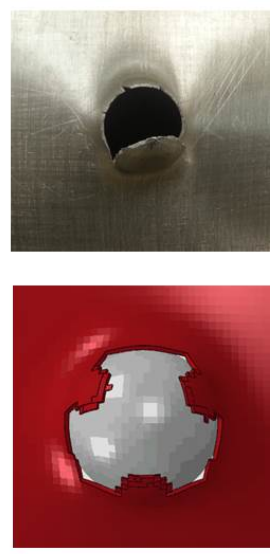

(b)

Figure 3.6: QSI response of bare aluminium plate of different thickness a) loaddeflection curve b) Indented damage surface

Figure 3.7 shows the variation of equivalent plastic strain $\left(\bar{\varepsilon}^{p}\right)$ along the length of $1 \mathrm{~mm}$ AL plate for different maximum flexural displacement $(w)$.It dictates that plastic deformation is extended throughout the plate length i.e. nowhere $\bar{\varepsilon}_{p}=0$ , and increases more rapidly under the contact region. Values of $\left(\bar{\varepsilon}^{p}\right)$ of elements around failure boundary confirm that elements those attaining $\bar{\varepsilon}_{p} \geq \bar{\varepsilon}_{p}^{f}$ are deleted from the cluster and ensures the accuracy of fracture energy values calibrated using equation (3.17).

Higher radial and circumferential tensile stresses induced by the indenter of hemispherical blunt indenter results in a formation of cylindrical plug of approximately the same diameter as the contact radius as shown in Figure 3.6(b). The comparisons between numerical and experimental results are quite in agreement both in shape and size.

\subsubsection{Low velocity impact response of bare aluminium plate}

Reliability of the constitutive model to impact load is studied by impacting $1 \mathrm{~mm}$ aluminium plate with impactor mass of $2.735 \mathrm{~kg}$ at $2 \mathrm{~ms}^{-1}$ impact velocity. Impact characteristics measured in terms of contact force versus time and energy versus time predicted by numerical models are compared with that of experiments in Figure 


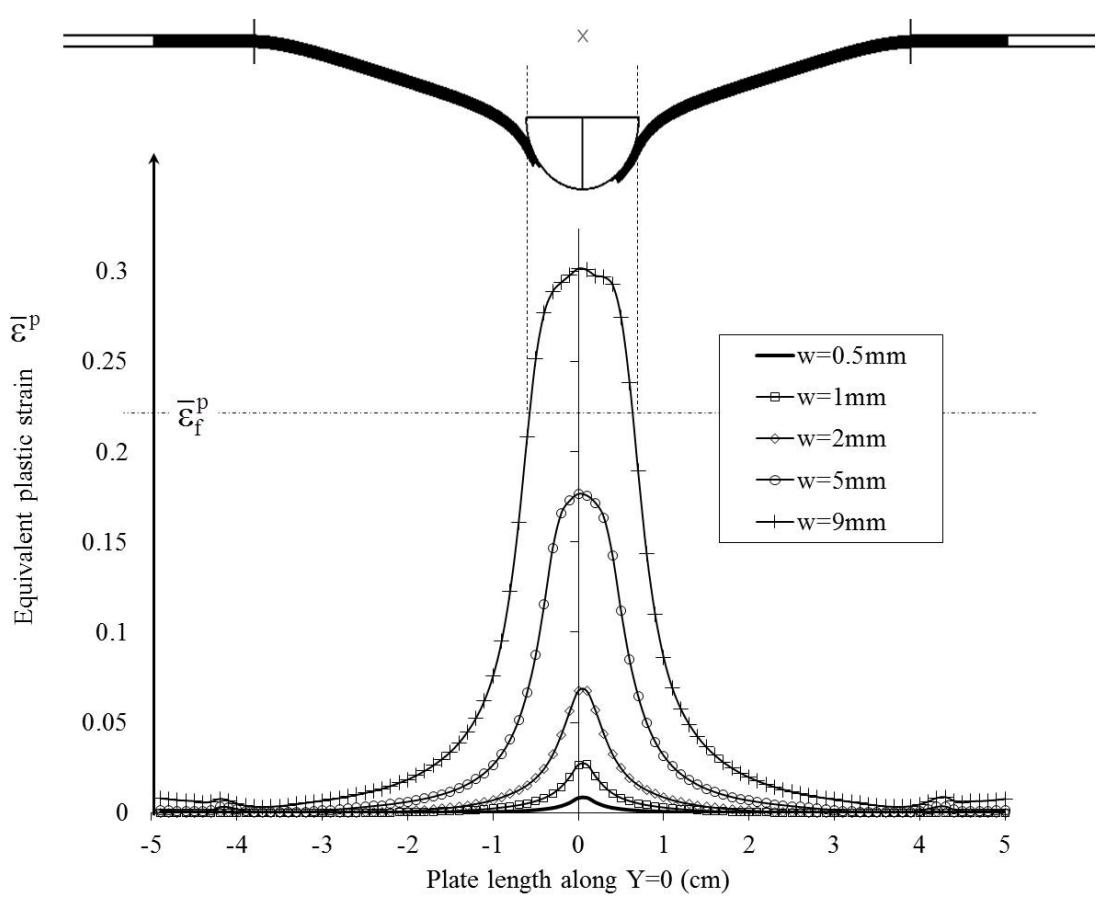

Figure 3.7: Variation of equivalent plastic strain $\left(\bar{\varepsilon}^{p}\right)$ of indented clamped circular metal plate

3.8 (a). Good correlation exists throughout ascending loading section and peak load predictions and the resultant energy absorbed registers only a difference of $5 \%$. The accuracy of simulation is estimated by calculating the energy balance magnitude during the contact time as shown in Figure 3.8(b). Contributions from spurious deformation modes given by hourglass energy is well below $1 \%$ of peak internal energy and ensure all the applied kinetic energy is transformed to internal energy of plate. The above statement is reconfirmed through total energy variation which is almost constant during the contact time. These preliminary studies confirmed that the stated constitutive model is good enough to predict the indentation and impact behaviour of metal layers

\subsection{Material model for FRP composite layer}

\subsubsection{Details of constitutive model}

The constitutive model used to define the behaviour of fabric FRP layer is shown in Figure 3.9. The initial undamaged response of fabric FRP layer is modelled as 

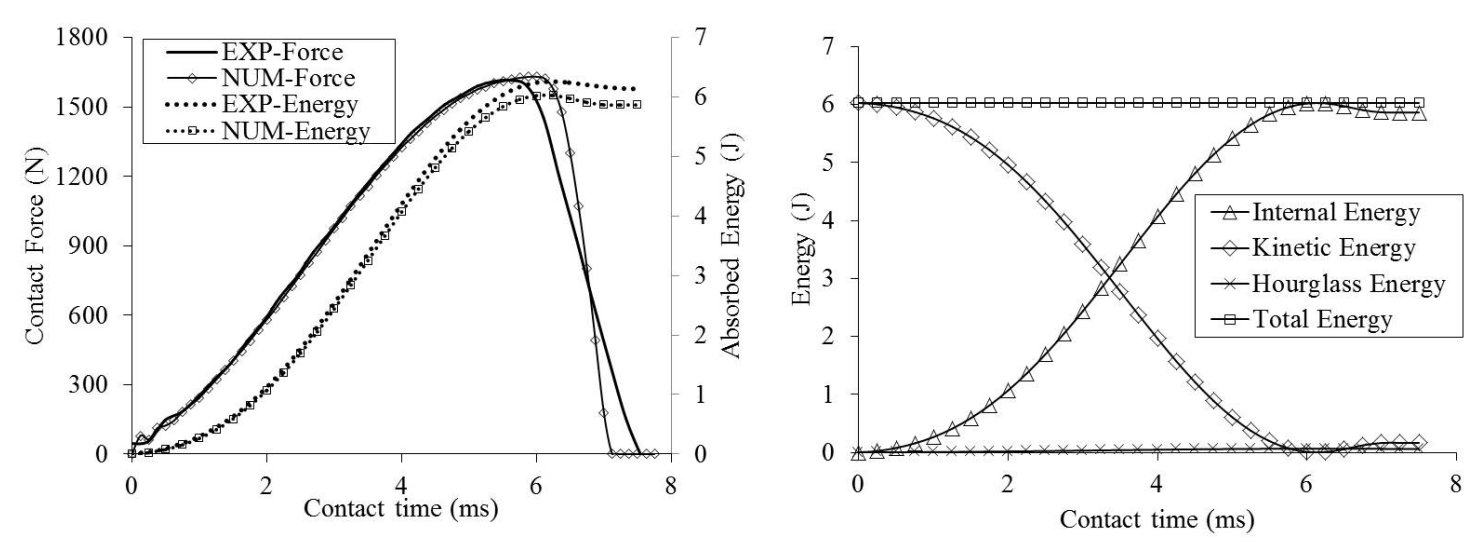

Figure 3.8: LVI response of bare aluminium plate a) Impact characteristic curves b) Energy balance plot

homogeneous, linear orthotropic elastic material defined by;

$$
\left\{\begin{array}{l}
\varepsilon_{11} \\
\varepsilon_{22} \\
\varepsilon_{12}
\end{array}\right\}=\left[\begin{array}{ccc}
\frac{1}{E_{1}^{0}} & \frac{-\nu_{12}}{E_{2}^{0}} & 0 \\
\frac{-\nu_{21}}{E_{1}^{0}} & \frac{1}{E_{2}^{0}} & 0 \\
0 & 0 & \frac{1}{2 G_{12}^{0}}
\end{array}\right]\left\{\begin{array}{l}
\sigma_{11} \\
\sigma_{22} \\
\sigma_{12}
\end{array}\right\}
$$

where $E_{1}, E_{2}, \nu_{12}$ and $G_{12}$ are the in-plane orthotropic elastic constants. The subscripts 1 and 2 denote warp and weft directions respectively.

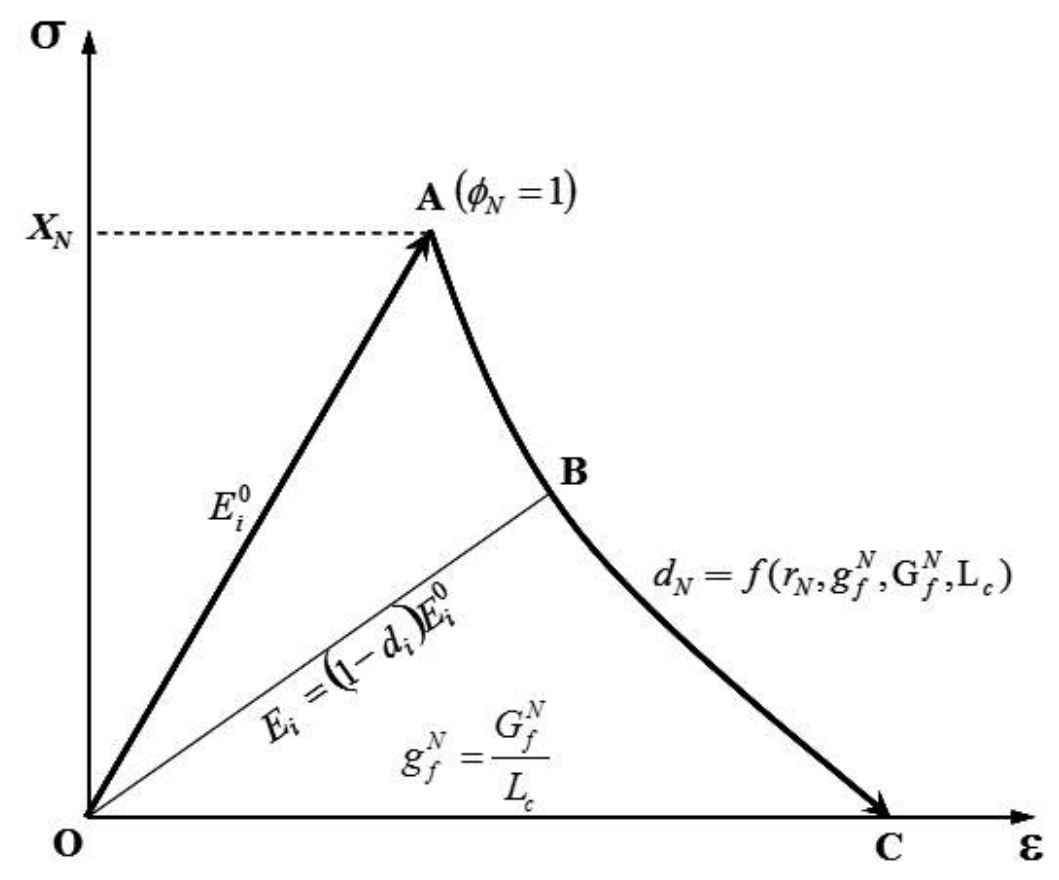

Figure 3.9: Constitutive material parameters of FRP layer 
Continuum damage mechanics (CDM) based intralaminar damage model is found to be an accurate methodology to predict brittle failure of FRP layer [64, 99]. The concept of CDM is based on "strain equivalence principle" initially proposed by Lemaitre 100 and states that "the constitutive relation of damaged material is usually derived in the same way as undamaged material except that the stress state in the damaged material is defined in terms of effective stress $(\bar{\sigma})$ " given by,

$$
\bar{\sigma}=D \sigma
$$

where, $D$ is the damage variable which is 0 at damage onset and 1 at final failure (zero stiffness).

In the present work, CDM based intralaminar damage model proposed by Johnson et.al [90] is used to predict the damaged response of fabric FRP layer. The implemented model accounts fibre fracture along warp, weft direction and matrix cracking due to in-plane shear loads. The constitutive equation of damage response is obtained through the definition of thermodynamic potential, where the complementary free energy density is defined by;

$$
G=\frac{\sigma_{11}^{2}}{2\left(1-d_{1}\right) E_{1}^{0}}+\frac{\sigma_{22}^{2}}{2\left(1-d_{2}\right) E_{2}^{0}}-\frac{\nu_{12}}{E_{1}} \sigma_{11} \sigma_{22}+\frac{\sigma_{12}^{2}}{2\left(1-d_{12}\right) G_{12}^{0}}
$$

By ensuring the thermodynamic irreversibility of damage process, the relationship between strain $(\varepsilon)$ - stress $(\sigma)$ of damaged material takes the form [59]:

$$
\begin{gathered}
\varepsilon=\frac{\partial G}{\partial \sigma}=H: \sigma \\
\left\{\begin{array}{l}
\varepsilon_{11} \\
\varepsilon_{22} \\
\varepsilon_{12}
\end{array}\right\}=\left[\begin{array}{ccc}
\frac{1}{\left(1-d_{1}\right) E_{1}^{0}} & \frac{-\nu_{12}}{E_{1}^{0}} & 0 \\
\frac{-\nu_{21}}{E_{2}^{0}} & \frac{1}{\left(1-d_{2}\right) E_{2}^{0}} & 0 \\
0 & 0 & \frac{1}{\left(1-d_{12}\right) 2 G_{12}^{0}}
\end{array}\right]\left\{\begin{array}{c}
\sigma_{11} \\
\sigma_{22} \\
\sigma_{12}
\end{array}\right\}
\end{gathered}
$$

where $H=\frac{\partial^{2} G}{\partial \sigma \otimes \partial \sigma}$ is the lamina compliance tensor, $d_{1}$ and $d_{2}$ are the scalar 
damage variables associated with fibre fracture along warp and weft directions respectively, whereas $d_{12}$ activate matrix cracking due to in-plane shear deformation.

The crack closure during the load reversal is taken in to account by separating damage induced due to tensile $(+)$ and compressive $(-)$ fibre failure modes with the definition of corresponding stress variables inside the McCauley operator $[\langle x\rangle:=$ $\left.\frac{(x+|x|)}{2}\right][16]$. Therefore, the formulation of fibre damage scalar variable is defined as;

$$
d_{i}=d_{i+} \frac{\left\langle\sigma_{i}\right\rangle}{\left|\sigma_{i}\right|}+d_{i-} \frac{\left\langle-\sigma_{i}\right\rangle}{\left|\sigma_{i}\right|}
$$

where $i=1,2$ for fibre failure in warp and weft direction respectively. Damage onset is defined in terms of damage activation function $\left(F_{\alpha}\right)$ given by;

$$
F_{N}=\phi_{N}-r_{N} \leq 0
$$

where, $N \epsilon(1 \pm, 2 \pm)$ accounts fibre failure along warp (1) and weft (2) directions under tension $(+)$ and compression $(-)$ load and $\phi_{N}$ is the limit load function or simply damage initiation function. In the present work, the limit load function for fibre failure is defined by the maximum principal stress criterion:

$$
\phi_{N}=\frac{\bar{\sigma}_{N}}{X_{N}}
$$

where $\bar{\sigma}_{N}$ and $X_{N}$ are components of the effective stress tensor $\bar{\sigma}$ and the corresponding ultimate strength along specified fibre direction respectively. The term $r_{N}$ in equation (3.24) is called damage threshold which defines a boundary between undamaged and damaged response that takes a value of 1 at the onset of damage and increases with respect to damage progress according to the relation;

$$
r_{N}(t)=\max \underset{\tau \leq t}{\phi_{N}(\tau)}
$$

The above form of relation satisfies the statement of the second law of thermody- 
namics by keeping $r_{N}$ as an increasing function at any instant of time $\left(\dot{r}_{N}(t) \geq 0\right)$ . This monotonically increasing $r_{N}$ is used to quantify scalar damage variable $d_{i}$ by means of damage evolution law of exponential form 101] defined as:

$$
d_{N}=1-\frac{1}{r_{N}} e^{\mid \frac{2 g_{f}^{N} L}{G_{f}^{N}-2 g_{f}^{N} L}\left(1-r_{N}\right)} \mid
$$

where, $g_{f}^{N}$ is the fracture energy per unit area and $G_{f}^{N}$ is the strain energy density for a particular loading case $N$.

Damage activation function, loading functions and damage threshold for shear damage of matrix takes similar expression as eqs. (3.24) to 3.26 respectively, where $N=12$. The evolution criterion for shear damage is modelled as sudden degradation due to smaller critical fracture energy of this failure mode, i.e. when $d_{12}=1$, the associated stiffness component vanish. Element deletion is activated when any $d_{i \pm}$ reaches a maximum specified value of 0.99 instead of reaching unity in order to avoid sudden distortions due to sudden zero element stiffness which frequently abort the simulations $[102$.

\subsubsection{Calibration of material parameters}

The material properties required to define the composite constitutive model are partially predicted by means of standard test methods while others are taken from manufacturer's database. Ply elastic properties $\left(E_{1}, G_{12}\right)$ and ply strength $\left(X_{1+}, S_{12}\right)$ properties $\left(E_{1}, X_{1+}\right)$ are measured using respective test standard described by the ASTM 103, 104].

There is no specific test method available to predict the fracture energy $G_{f}^{N}$ associated with fibre fracture in tension and compression stress medium. Pinho et.al [105], proposed a kind of compact tension (CT) and compact compression (CC) test method to measure $G_{f}^{N+}$ and $G_{f}^{N-}$ respectively. The determined values ranged between $91 \mathrm{~N} / \mathrm{mm}$ to $133 \mathrm{~N} / \mathrm{mm}$ for carbon/epoxy system. For glass/epoxy system, a range of $30 \mathrm{~N} / \mathrm{mm}$ to $50 \mathrm{~N} / \mathrm{mm}$ was recorded through similar test [106. 
These measured values were found to be highly dependent on layup angle and not constant for a given material. Moreover, the influence of mesh size in FE simulation (via. Characteristic element length $L$ ) makes the selection of appropriate $G_{f}^{N}$ more ambiguous.

In the present work, a series of trial simulations has been performed to select appropriate $G_{f}^{N}$ value for a given mesh size based on the predictions of peak load. It was noticed that fracture energy required to correlate the experiment results were found to be different for indentation and impact simulations. This might be because of the high rate dependent characteristics of glass fibres. The material input properties used to represent FRP constitutive model in the simulations are listed in Table 3.3 .

Table 3.3: Material properties of Hexply 7781/L-530 GFRP prepeg

\begin{tabular}{lll}
\hline Response & \multicolumn{1}{c}{ Property } & \multicolumn{1}{c}{ Value } \\
\hline \multirow{4}{*}{ Elastic } & Density & $\rho=1100 \mathrm{~kg} / \mathrm{m}^{3}$ \\
& In-Plane tensile Young's Modulus & $E_{1+}=E_{2+}=24 G \mathrm{~Pa}$ \\
& In-Plane compression Young's Modulus & $E_{1-}=E_{2-}=24 G \mathrm{~Pa}$ \\
& Shear Modulus & $G_{12}=3.6 \mathrm{GPa}$ \\
\hline \multirow{2}{*}{ Damage initiation } & Ultimate tensile strength & $X_{1+}=X_{2+}=414 \mathrm{MPa}$ \\
& Ultimate compression strength & $X_{1-}=X_{2-}=458 \mathrm{MPa}$ \\
& Ultimate shear strength & $S_{12}=105 \mathrm{MPa}$ \\
\hline \multirow{2}{*}{ Damage evolution } & Fibre fracture energy* & $G_{1+}=G_{1-}=50(20) \mathrm{N} / \mathrm{mm}$ \\
& & $G_{2+}=G_{2-}=50 \mathrm{~N} / \mathrm{mm}$ \\
\hline
\end{tabular}

${ }^{*}$ Value inside the parentheses is used for quasi static indentation simulation.

\subsubsection{Finite element analysis}

\subsubsection{Indentation response of woven FRP composite plate}

Indentation response (load versus deflection) and its corresponding indentation damage photograph of FRP plate are shown in Figure 3.10. Fracture energy in tension $G_{f}^{N+}$ shows strong influence on overall response and the predicted peak load increases with increase in $G_{f}^{N+}$. For the given mesh size, FE model with $G_{f}^{N+}=20 \mathrm{~N} / \mathrm{mm}$ shows good agreement in predicting the contact load and further softening process. However the ascending loading region is slightly stiffer than experimental curve and this might be because of plane stress assumption 
$\left(\sigma_{33}=0, \sigma_{13}=0, \sigma_{23}=0\right)$. Notwithstanding, the thickness of FRP layer in FML panels are very small and in this case the plane stress assumption associated with continuum shell modelling strategy is still valid enough to predict key characteristic response as confirmed from the current comparative study.

The failure mode is mainly fibre breakage along warp and weft direction due to axi-symmetric structure as shown in Figure 3.10(b). Two mutually perpendicular line cracks nucleated under the contact point and propagated towards the boundary. Crack dimensions observed from FE simulation correlate rather well with experimental observations.

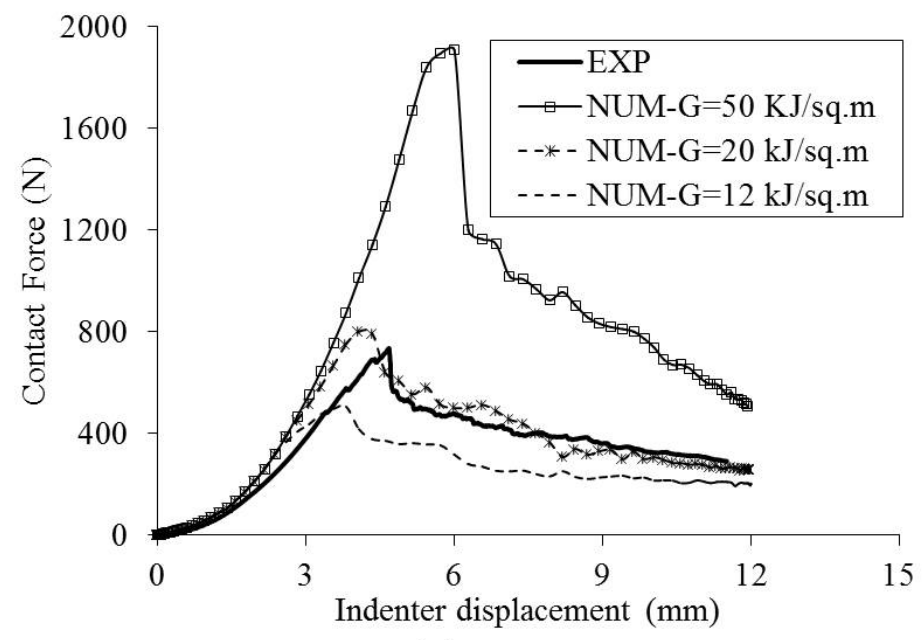

(a)
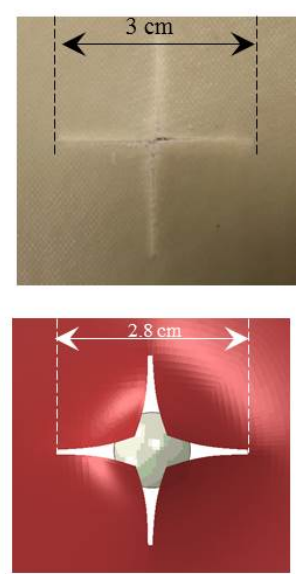

(b)

Figure 3.10: QSI response of monolithic FRP plate a) load-deflection curve showing effect of fracture energy of fibre b) Indented damage surface

\subsubsection{Impact response of woven FRP composite plate}

In a similar fashion to indentation response, FE simulated impact response also shows stiffer response during initial ascending loading region as shown in Figure 3.11. The parametric study reveals a value of $G_{f}^{N+}=50 \mathrm{~N} / \mathrm{mm}$ is needed to predict peak load and post damage response with a reasonable accuracy. The difference in $G_{f}^{N+}$ value between QSI and LVI simulations is due to any of the current simplification implemented; strain rate dependency, homogenized meso-modelling, discretization using shell elements. However, observations of global impact response, failure initiation load and contact time predictions are well comparable. With the selection 


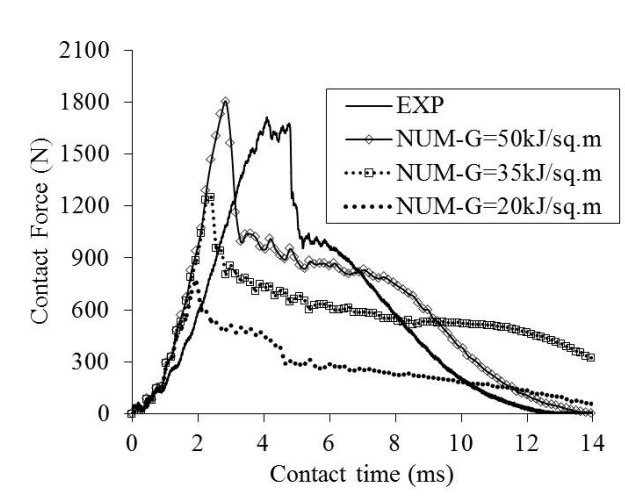

(a)

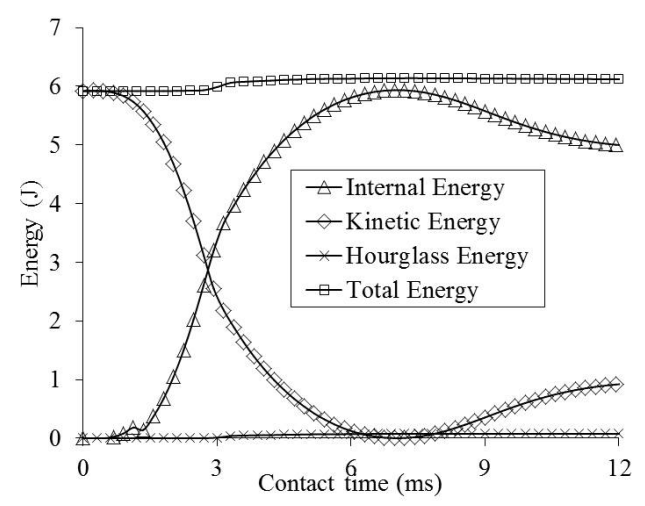

(b)
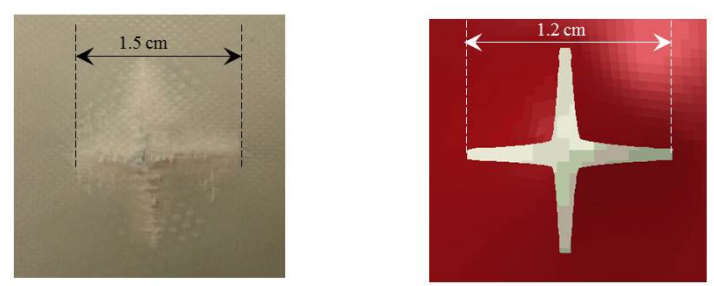

(c)

Figure 3.11: LVI response of monolithic FRP plate a) Contact force history b) Energy balance plot c) Impact damage surface

of enhanced hourglass control option, percentage of energy dissipated through this mode is highly suppressed and less than $10 \%$ of internal energy as manifested from the energy balance plot in Figure 3.11(b). For the impact load case, the failure mode is dominated by fibre breakage having symmetric mutually perpendicular line crack along weft and warp directions as shown in Figure 3.11k. The general numerical solution using the stated constitutive model is comparable not only qualitatively but also quantitatively with experimental measurements both for indentation and impact loads.

\subsection{Material model for interlaminar region}

Delamination/De-bonding is one of the common failure modes in laminated composite materials subjected to low velocity impact load. Linear Elastic Fracture Mechanics (LEFM) based methods like virtual crack closure technique (VCCT), virtual crack extension, J-integral has been proven to be an effective numerical method in predicting delamination growth if the crack location is pre-known. If no pre-cracks are present, cohesive crack model based on the frame work of damage mechanics is 
found to be powerful method in capturing delamination. It considers interlaminar region as an independent material defined by its own constitutive material model comprising delamination initiation and successive evolution formulations [107]. All the numerical simulation in the present work uses the inbuilt three dimensional cohesive elements (COH3D8) available in ABAQUS FE code to model interlaminar behaviour. Details of the constitutive model and calibration procedures are narrated in the following sections.

\subsubsection{Constitutive behaviour}

Unlike constitutive relations defined by stress versus strain tensors, cohesive crack model relates stress (traction) tensors $t_{\alpha}$ to corresponding displacement tensors $\delta_{\alpha}$. The constitutive response of cohesive elements used here is bi-linear in nature as shown in Figure 3.12. The initial linear elastic response prior to damage onset $(\mathrm{OA})$ is characterized by three stiffness parameters related as;

$$
\left\{\begin{array}{l}
t_{n} \\
t_{s} \\
t_{t}
\end{array}\right\}=\left[\begin{array}{ccc}
K_{n n} & 0 & 0 \\
0 & K_{s s} & 0 \\
0 & 0 & K_{t t}
\end{array}\right]\left\{\begin{array}{c}
\delta_{n} \\
\delta_{s} \\
\delta_{t}
\end{array}\right\}
$$

where $\alpha \epsilon n, s, t$ represent normal and two orthogonal shear components. The normal and shear tractions are considered not to be coupled in initial elastic regime and so stiffness tensor $K_{i j}=0$, if $i \neq j$. Damage onset $\left(\delta_{\alpha}^{0}, t_{\alpha}^{0}\right)$ is related to interface strength $t_{\alpha}^{0}$ when the stress in the cohesive layer reaches the quadratic strength criterion given by:

$$
\left\{\frac{\left\langle t_{n}\right\rangle}{t_{n}^{0}}\right\}^{2}+\left\{\frac{t_{s}}{t_{s}^{0}}\right\}^{2}+\left\{\frac{t_{t}}{t_{t}^{0}}\right\}^{2}=1
$$

where, the Macaulay bracket $\langle\bullet\rangle$ ensures normal compressive stress does not contribute to damage initiation. The linear softening behaviour $\left(\delta_{\alpha}^{0}, t_{\alpha}^{0} \longrightarrow \delta_{\alpha}^{f}, t_{\alpha}^{f}\right)$ of cohesive de-bonding after the damage initiation (path AB in Figure 3.12) is defined 


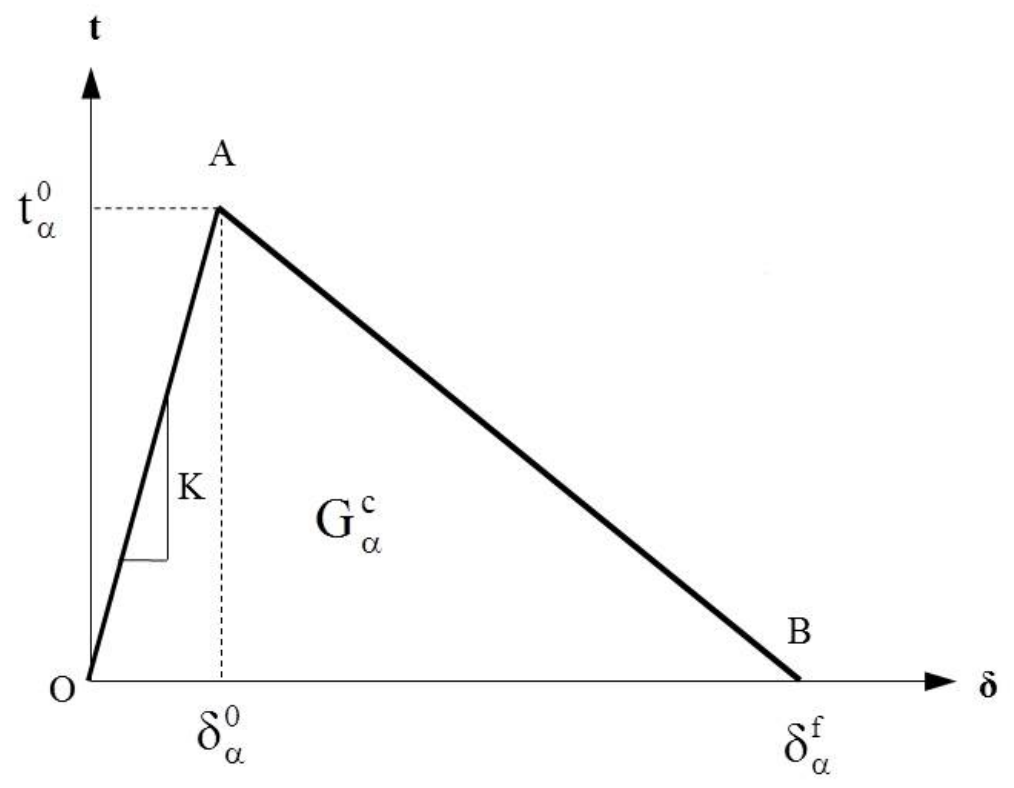

Figure 3.12: Constitutive material input parameters of cohesive interface layer in terms of critical fracture energy $\left(G_{\alpha}^{c}\right)$ based on the relation:

$$
\left\{\frac{G_{n}}{G_{n}^{c}}\right\}^{\eta}+\left\{\frac{G_{s}}{G_{s}^{c}}\right\}^{\eta}+\left\{\frac{G_{t}}{G_{t}^{c}}\right\}^{\eta}=1
$$

where, $\eta$ denotes the magnitude of exponent defining the variation of fracture energy with mode mix. For the linear softening behaviour used in the present work, $\eta$ takes a value of 1 .

\subsubsection{Calibration of constitutive model}

Unlike other structural elements, the behaviour of cohesive element is numerically more sensitive to input parameters and often require intense care in the calibration stage. The behaviour of interfacial cohesive elements is characterized by three independent input parameters: penalty stiffness $(K)$ to determine initial compliance, Interface strength $\left(t_{\alpha}^{0}\right)$ to model damage initiation, critical fracture toughness $\left(G_{\alpha}^{c}\right)$ to control damage progression.

\subsubsection{Penalty stiffness}

The stiffness contribution to effective elastic properties and global deformation of laminates should be small since, the purpose of cohesive layer in the laminated material is to simulate interface fracture. Different guidelines have been present 
in literature for selecting appropriate penalty stiffness $(K)$ of cohesive elements. They are either material property dependent [108, 109] or empirical [110 and the proposed values covers wide range from $10^{4} \mathrm{~N} / \mathrm{mm}^{3}$ to $10^{7} \mathrm{~N} / \mathrm{mm}^{3}$. In the present work, a method proposed by Turon [111 based on effective Young's modulus $\left(E_{\text {eff }}\right)$ of laminated composite material is used to select $K$. For a cohesive material of stiffness $K$ bonded between composite layer having transverse stiffness $\left(E_{3}\right)$ and thickness $(t)$, the expression for $E_{\text {eff }}$ takes the form of:

$$
E_{e f f}=E_{3}\left(\frac{1}{1+\frac{E_{3}}{K t}}\right)
$$

From above equation, the influence of cohesive stiffness $K$ to $E_{\text {eff }}$ becomes passive whenever $E_{3}<<K$, and this leads to;

$$
K=\frac{\alpha E_{3}}{t}
$$

where $\alpha$ is the constant parameter usually takes a value larger than unity. In this work, a value of 50 is used [27].

\subsubsection{Interface strength}

From Alfano et.al 112 it is understood that interfacial strength parameters $t_{\alpha}^{0}$ of cohesive elements do not have strong influence on resulted delamination initiation response. In the present work, a value of $50 \mathrm{MPa}$, an approximate tensile strength of typical epoxy adhesive is used as a critical interfacial strength parameter to initiate delamination damage by all fracture modes.

\subsubsection{Critical fracture toughness}

For extremely thin adhesive regions, normal fracture mode is extremely crucial and its fracture toughness $G_{n}^{c}$ is generally $50 \%$ smaller compared to other two modes [113, 114]. Thus, calibrating mixed mode damage evolution law of equation (3.30) solely by $G_{n}^{c}$ seems reasonable enough to simulate de-bonding fracture surface of 
MCI region of FML panels.

Using standard double cantilever beam (DCB) test, determination of $G_{n}^{c}$ for the adhesive interface with similar substrates are straightforward [115, 116]. However for multi-material substrates, no specific standard exists to calculate true $G_{n}^{c}$ and impose even more difficulty if the substrates contain thin metal sheets because plastic flow of thin metal sheets will obscure and perhaps produce abnormally high fracture energy values. The uncertainty is more pronounced if one attempts to determine the $G_{n}^{c}$ of MCI region in FML since, the panel is made of thin metal and FRP layers.

To estimate the true fracture toughness for this kind of complex geometry, several approaches had been proposed in literature: (i) Experimental approach using thick substrates [117 119] to minimize metal layer plastic flow (ii) Theoretical approach [120] and, (iii) Finite element (FE) approach. In the last two approaches, efforts were made to estimate fraction of plastically absorbed energy from overall internal energy at the time of fracture. In the present work, FE approach is utilized to determine the fracture toughness of MCI interface formed between thin metal and composite substrates.

Two types of adhesive systems were employed to bond metal and FRP layers of FML in the present work: (i) Self-adhesive from FRP prepreg itself (ii) Redux $335 \mathrm{~K}$ adhesive epoxy adhesive film to promote enhanced bonding strength. In the following paragraphs, characterization results for the self adhesive system is briefly detailed in the subsequent section and the predicted quantitative values for Redux $335 \mathrm{~K}$ adhesive system using similar method is listed in Table 3.4 .

\section{Mode-I fracture testing of DCB samples with similar substrates:}

As a benchmark, the fracture toughness using homogeneous FRP/FRP composite substrate was estimated based on the standard described in ASTM D5528 [116].Numerical simulations were performed to check the reliability of FE model in computing the fracture energy. The interfacial region is discretized with cohesive element and the fracture energy obtained from DCB experiments is used as an input material parameter for cohesive layer and material properties from Table 3.3 is used to 
model FRP composite layer. It has been reported that mesh density of cohesive elements shows significant influence on overall fracture response of FE simulations 121. Methodology to achieve optimum mesh-size is briefly discussed in Appendix A. The following paragraph reports only the optimum mesh results and comparison with experiment results.

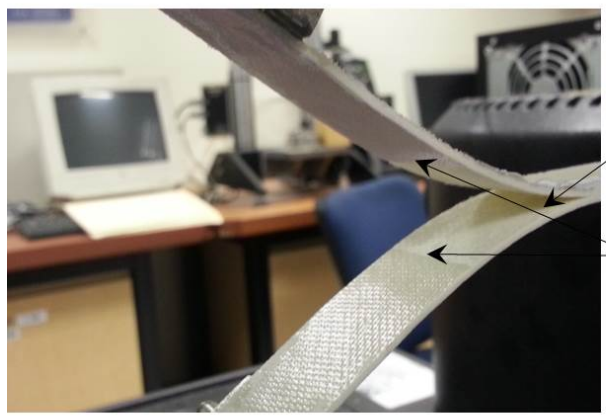

a)

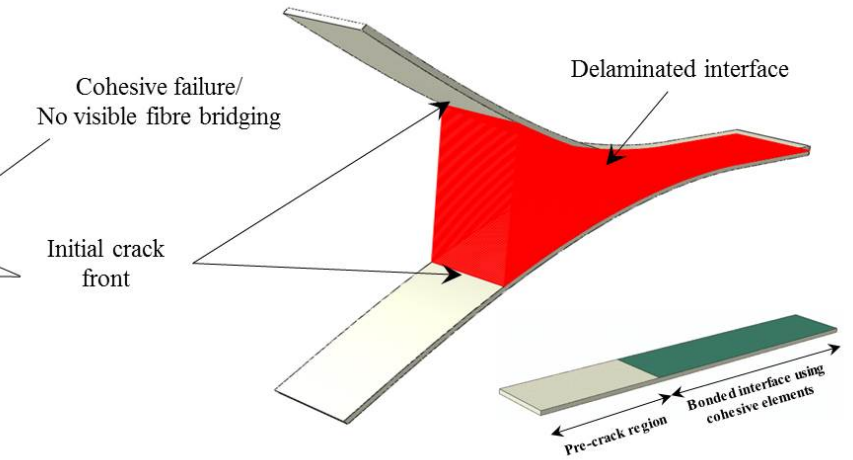

b)

Figure 3.13: Mode-I DCB fracture test a) Fracture surface of FRP composite substrate b) FE model

The fracture surface of experiment show the complete cohesive fracture path through the adhesive layer and no evidence of fibre bridging interventions involved to arrest crack propagation due to weave nature of woven fabric as shown in Figure $3.13(\mathrm{a})$.

The modelling details of interfacial region using cohesive elements and corresponding simulated fractured shape is shown in Figure 3.13(b). Experimental fracture energy values are ascertained directly using Irwin-Kies equation 122;

$$
G_{n}^{c}=\frac{P^{2}}{2 B} \frac{d c}{d a}
$$

where $C$ is the compliance given by displacement of substrate $\delta$ divided by applied load $P$ and $B$ is width of the beam. The FE fracture energy values are ascertained using area method 123 :

$$
G_{n}^{c}=\frac{\Delta U}{B \Delta a}
$$

where $U$ is the area under the $P-\delta$ trace and $\Delta a$ is the crack length increment 
a)

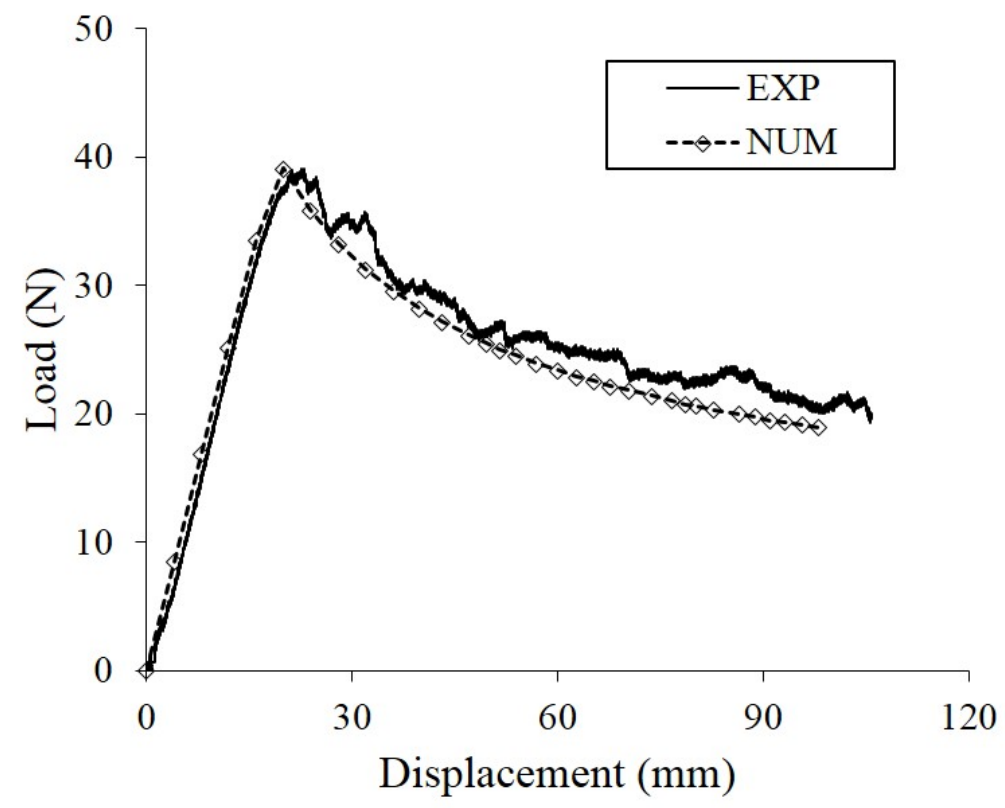

b)

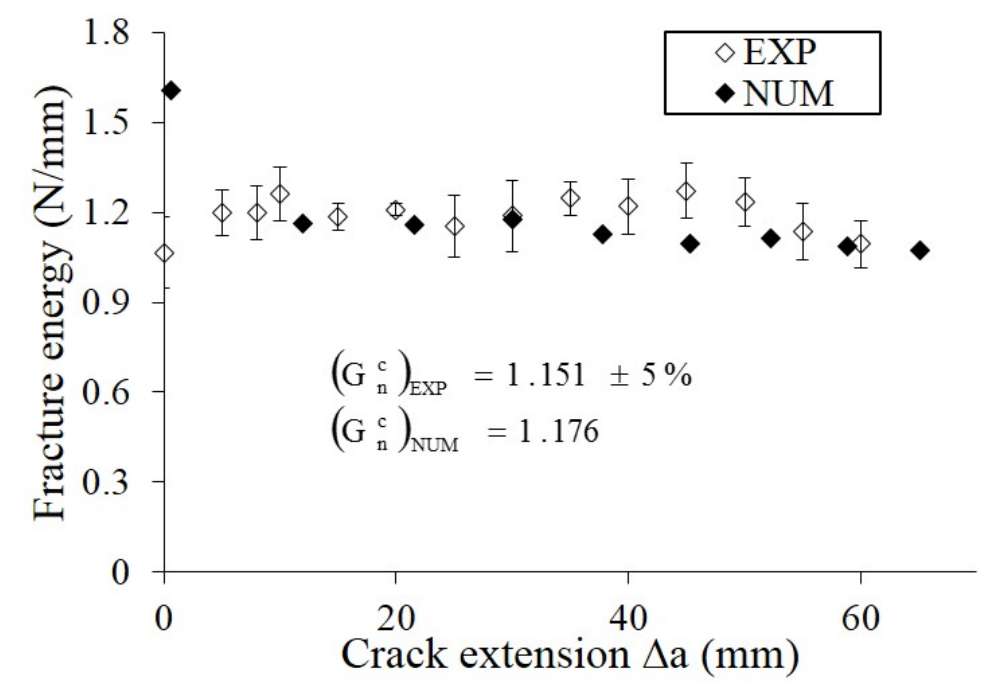

c)

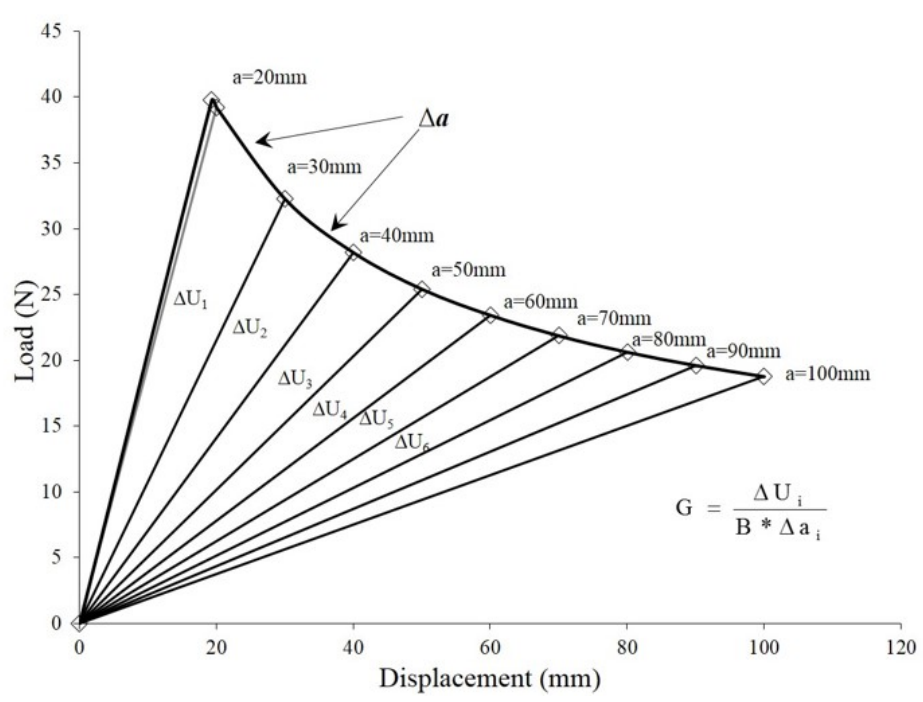

Figure 3.14: Mode-I fracture test with homogeneous FRP substrate a) Loaddeflection curve b) R-curve c) Area method to determine $G_{c}$ 
as shown in Figure 3.14(c). Comparative results between experiment and numerical predictions in terms of load-displacement and R-curve as shown in Figure 3.14(a) and Figure 3.14(b) confirms that the designed FE model is able to propagate the initiated pre-crack at pre-stated fracture energy.

\section{Mode-I fracture testing of DCB samples with dissimilar substrates:}

From the mechanics stand point, the deflection of DCB specimens with dissimilar substrates inherently induce mixed mode loading state on the pre-crack front due to un-symmetric flexure of the un-bonded portion of the DCB arm. To attenuate the mode-mixity interference in such design, both substrates need to be constructed in a manner such that,

$$
(E I)_{\text {topsubstrate }}=(E I)_{\text {bottomsubstrate }}
$$

where $E I$ is the flexural rigidity. Employing elementary mechanics, the thickness required for each material substrate to achieve similar EI is determined [119] and substrates are fabricated according to that particular dimension. The schematic of the tested geometry is shown in Figure 3.15. In the FE simulation, a tie constraint

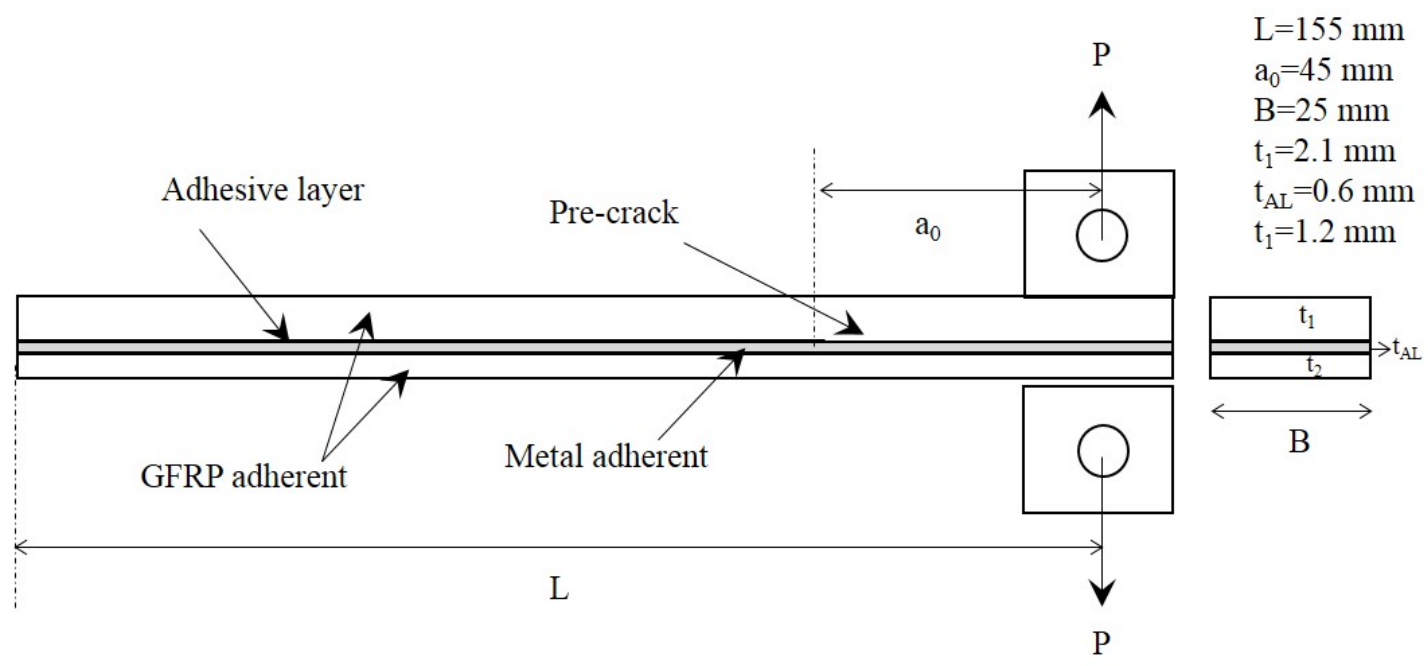

Figure 3.15: Mode-I DCB fracture test sample with dissimilar substrates

has been adopted to replicate firm bonding of heterogeneous substrate containing metal and FRP layer. The region with pre-crack between dissimilar substrate is 
discretized with cohesive elements. The material data to model the plastic response of the metal layer is taken from Table 3.2 .

With the increase in crack opening displacement, the nature of the crack growth in experiments is found to change from stable to unstable manner. The continuous cumulative effect of plastic flow in metal layers makes the crack growth as stick slip manner in the form of periodic short bursts and crack arrest. This scenario leaves a combined cohesive and adhesive kind of fracture surface along the MCI region. Figure 3.16(a) shows a typical load-displacement curve obtained from experiment and FE numerical simulations. Predictions of peak load and initial softening response are well correlated with each other while there is notable difference in the latter stage and it might be because crack propagation in FE simulation is always modelled as stable and cohesive.

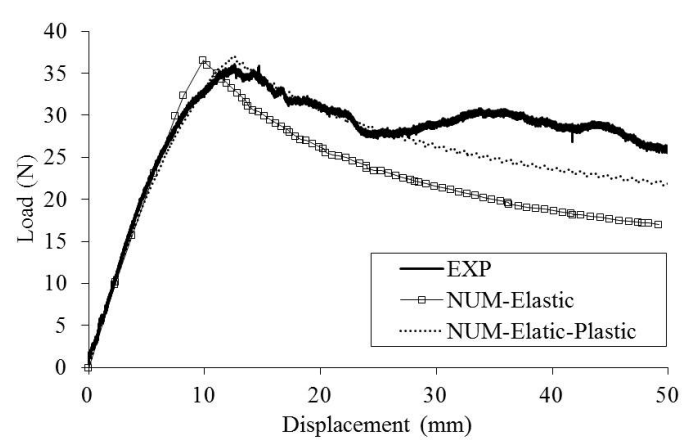

a)

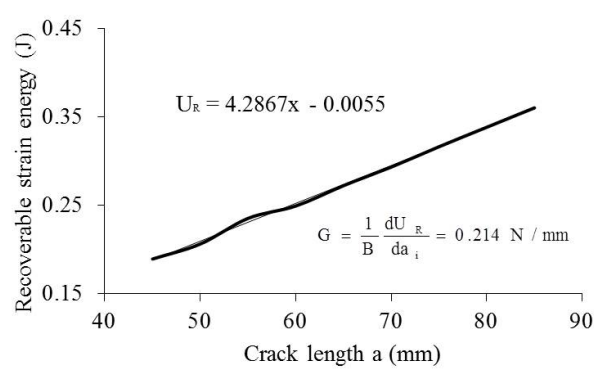

c)

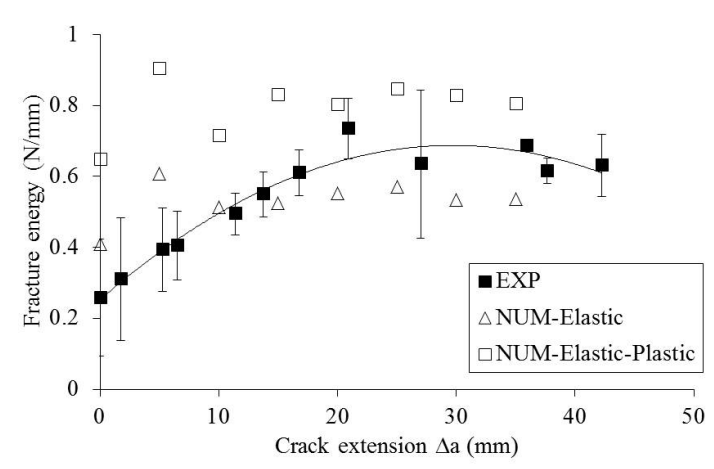

b)

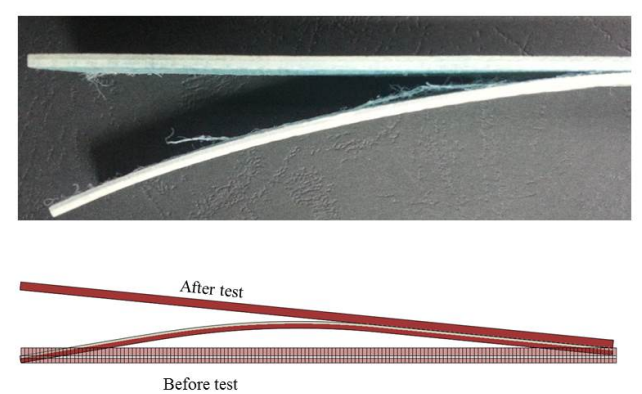

d)

Figure 3.16: Mode-I fracture test with heterogeneous FRP substrate a) Loaddeflection curve b) R-curve c) Recoverable strain energy versus crack length d) Permanent deformation of DCB substrate-Experiment and FE simulation

Using equations (3.33) and (3.34), the respective fracture energy values from experiments and FE simulations are calculated as plotted in Figure 3.16(b). These 
values are not a true fracture energy values utilized to propagate the pre crack and it includes plastically absorbed energy by metal layers as well. This is manifested from permanent deformed shape of tested samples as shown in Figure 3.16(d). Numerically, it seems that plastic flow of metal substrates over predicted the fracture energy at least by a factor of 1.5 when comparing the numerical results obtained from pure elastic $\left(G_{n}^{c}\right)_{\text {Elas }}$ and combined elastic plastic simulation $\left(G_{n}^{c}\right)_{\text {Elas-Plas }}$ as shown in Figure 3.16(b). However these values are based on input obtained from experiments and did not provide exact fracture energy value.

Table 3.4: Summary of predicted fracture energy values

\begin{tabular}{|c|c|c|c|c|}
\hline $\begin{array}{l}\text { Fracture } \\
\text { energy }\end{array}$ & $\begin{array}{l}\operatorname{EXP}\left(\mathrm{Eq}^{-}\right. \\
(3.33)\end{array}$ & $\begin{array}{l}\text { FE-Elastic(Eq- } \\
(3.34)\end{array}$ & $\begin{array}{l}\text { FE-Elastic-plastic } \\
\text { (Eq- (3.34) }\end{array}$ & $\begin{array}{l}\text { FE-Elastic-plastic } \\
(\text { Eq- (3.36) })\end{array}$ \\
\hline \multirow{2}{*}{$G_{n}^{c}(\mathrm{~N} / \mathrm{mm})$} & $\left(G_{n}^{c}\right)_{I n i}=0.257$ & \multirow{2}{*}{$\left(G_{n}^{c}\right)_{E l}=0.529$} & \multirow{2}{*}{$\left(G_{n}^{c}\right)_{E l-P l}=0.796$} & \multirow{2}{*}{$\left(G_{n}^{c}\right)_{\text {True }}=0.214$} \\
\hline & $\left(G_{n}^{c}\right)_{\text {Prop }}=0.595$ & & & \\
\hline
\end{tabular}

True fracture energy value can be calculated by extracting elastic strain energy magnitude from the FE results and substitute to equation (3.36) similar as equation (3.34);

$$
G_{n}^{c}=\frac{\Delta U_{R}}{B \Delta a}
$$

where, $U_{R}$ is the recoverable (or elastic) strain energy obtained using ABAQUS output variable ALLSE in post-processing. By plotting $U_{R}$ versus $a$ as shown in Figure 3.16(c) and taking the derivative, $\left(G_{n}^{c}\right)_{\text {True }}$ can be found from equation (3.36). The values of fracture energy obtained from different methods are summarized in Table 3.4. The obtained $\left(G_{n}^{c}\right)_{\text {True }}$ value is approximately equal to the initiation fracture energy $\left(G_{n}^{c}\right)_{i n i}$ predicted by experiments but it is $50 \%$ smaller than the propagation fracture energy $\left(G_{n}^{c}\right)_{\text {prop }}$ due to the notable difference in crack propagation path and plasticity effects.

With these detailed characterization study, the material input parameters selected to define cohesive interfacial region in the present work are tabulated in Table 3.5, 
Table 3.5: Properties of metal composite interface cohesive layer

\begin{tabular}{lll}
\hline Response & \multicolumn{1}{c}{ Property } & \multicolumn{1}{c}{ Value } \\
\hline Elastic & Penalty stiffness & $K_{n n}=K_{s s}=K_{t t}=1 e^{15} N / m^{3}$ \\
Damage initiation & Interface strength & $t_{n}^{0}=t_{s}^{0}=t_{t}^{0}=50 \mathrm{MPa}$ \\
& & $G_{n}^{c}=G_{s}^{c}=G_{t}^{c}=0.3 \mathrm{~N} / \mathrm{mm}$ (self) \\
\cline { 3 - 3 } Damage evolution & Fracture toughness & $G_{n}^{c}=G_{s}^{c}=G_{t}^{c}=0.75 \mathrm{~N} / \mathrm{mm}$ (Redux) \\
\hline
\end{tabular}

\subsection{Summary}

The configuration of the experimental methods and design framework of numerical FE models used in the present work to obtain quasi static indentation and low velocity impact response are presented. Mathematical formulations of three noninteractive constitutive material models governing the behaviour of isotropic metal, orthotropic FRP composite and cohesive interface layer in FE simulation are detailed. Calibration methods of constitutive models through appropriate standard test methods and series of trial FE simulations are also discussed.

Experimental and numerical DCB test were performed to measure the fracture toughness of the interface region formed by metal and composite substrates. Having thin metal sheets in one of the DCB arm, the measured fracture energy of interface was found over predicted by a factor of 1.5 due to plasticity effects. Retrieving the recoverable strain energy (elastically absorbed energy) through FE simulation, the true fracture energy of interface formed by plastically deformed substrate is measured with reasonable accuracy.

By phenomenology, this measurement should allow to model the de-bond shape and size of MCI region in FML panels. This is important major life-limiting failure mode under indentation and impact load where the integrity of structure is suddenly degraded. The capability of constitutive relations and reliability of FE model to simulate indentation and impact behaviour observed in experiments are validated for metal and FRP composite layer. The simulated characteristic response and predicted failure mode shapes are in good agreement with those found in experiments. 


\section{Chapter 4}

\section{Impact experiments on transparent plastics - A similitude model for FML}

This chapter presents the low velocity impact experiments of bi-layered transparent hybrid plastic specimens made of brittle acrylic sheet adhesively bonded with ductile polycarbonate sheet. Depending on the nature of impact face battered by the impactor either on brittle or ductile material, the corresponding impact event is classified either as hard or soft side impact respectively. The influence of relative constituent thickness towards impact damage resistance and the resultant structural integrity are extensively discussed. Specimen configurations are briefed in Section 4.2. The influence of impact face to the resultant impact response in terms of load-displacement and energy-displacement curves are investigated in Section 4.3 . Besides, the details of distinct damage modes prevails in the impacted samples and its influence on overall performance are discussed in Section 4.4. These understanding can be used to investigate the role of brittle and ductile material layer in the bonded bi-layered transparent hybrid. With this information, further attention is to be paid to extract influence and consequences of material constituents of equivalent GLARE FML in Chapter 5 . 


\subsection{Introduction}

In engineering applications, structures made of heterogeneous composition fulfill majority of anticipation in terms of robustness, reliability and durability. Like polymeric composites, materials such as ceramic, glass and acrylic or polymethyl methacrylate (PMMA) are naturally brittle in nature and often susceptible to fracture. Over many decades, several hybridization concepts have been introduced to improve the fracture behaviour of these brittle materials. Nowadays, fracture resistant transparent plastics are widely used as an enclosure for canopies and wind shields in aircraft and utilized as armors with high optical clarities in military in order to protect against armor-piercing and riot control applications. For better protection the current design armors carry two layers, the front layer of armor is made as hard as possible to damage the projectile and mount high stiff, tough backing layer to catch the residual projectile fragments and hindering the crack propagation by interface layer 124 . Thus most of the hybridization approaches involve interply addition of ductile layers, similar as concept of FML in an aim to achieve superior fracture protection 125 127.

Illinger et al. 128 performed an experimental impact event to understand the performance of acrylic/polycarbonate (PC) bonded bi-layer plastic composite over monolithic PC. In the course of evaluation, the impact resistance of acrylic/PC composite was found greatly enhanced than monolithic PC which was considered to be the best transparent impact resistant material at that period. Similar study related to acrylic/PC composite plate was conducted by Stenzler et al. [129]. They investigated the influence of different micro structurally varied interface media under transverse impact event. The presence of modified soft interlayer was found to exhibit enhanced energy absorption and provide improved impact resistance due to localization of fracture area and displayed a great degree of constraint towards crack growth 129, 130.

Liaw et.al [131, 132] attempted to enhance the fracture resistance of acrylic by interspersing aluminium layers. Experimental and numerical investigation of drop 
weight impact response on monolithic acrylic and hybrid acrylic/aluminium laminates was performed. Recently, $\mathrm{Hu}$ et al. 133 conducted a low velocity impact study on PC sandwiched glass laminates and to evaluate the dependence of damage pattern of glass towards impact velocity.

With the knowledge of these impact studies on ductile/brittle hybrid transparent plastics, it is of interest here to understand the impact behaviour of FML via comparing the characteristic response and fracture surfaces of equivalent ductile/brittle plastics. With lower material cost, fabrication costs and showing transparent fracture surfaces, such similitude methodology will provide a valuable preliminary information to the designers on role of material constituents in FML impact response.

\subsection{Experiment configuration and structure}

In the present similitude experiments, acrylic thickness between $1 \mathrm{~mm}$ to $4 \mathrm{~mm}$ was adhered firmly using 3M Scotch-Weld DP-100 room temperature cured epoxy adhesive with $1 \mathrm{~mm}$ thick PC plate. The fabricated bi-layer plastic laminates were categorized in terms of polycarbonate volume fraction defined as volume of PC to volume of complete laminate. In addition, each category encapsulates two different morphologies depend on impact face side. For convenience, the brittle acrylic side and ductile PC side impact samples are here after designated as hard and soft side impact samples respectively.

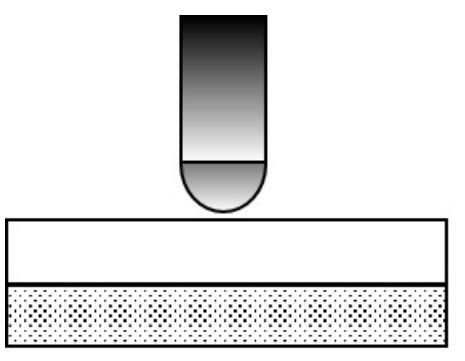

(a) Hard side Impact

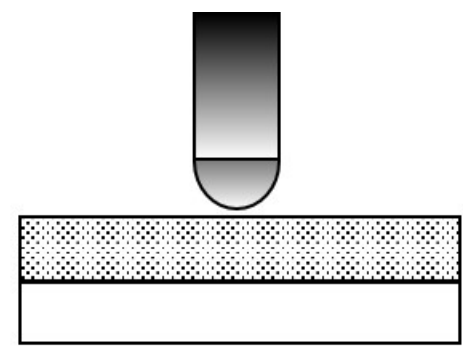

(b) Soft side Impact

$\square$ Acrylic (Brittle) layer

Polycarbonate (Ductile) layer

Figure 4.1: Schematic diagram of experimented bi-layered plastic samples

The schematic illustrations of experimental configuration are shown in Figure. 
4.1 and their corresponding geometric details are tabulated in Table 4.1. An impact velocity of $2 \mathrm{~m} / \mathrm{s}$ corresponds to impact energy of $6 \mathrm{~J}$ was utilized throughout the study. Other impact experiment configurations are same as given in section 3.1.2.

Table 4.1: Experimental structure to investigate acrylic/PC plastics

\begin{tabular}{cccccc}
\hline \multirow{2}{*}{ Sample } & \multicolumn{3}{c}{ Thickness $[\mathrm{mm}]$} & \multirow{2}{*}{ PVF } & weight $\left[\mathrm{kg} / \mathrm{m}^{2}\right]$ \\
\cline { 2 - 4 } & Polycarbonate & Acrylic & Total panel & & \\
\hline $\mathrm{PVF}=0.5$ & 1 & 1 & 2 & 0.5 & 2.46 \\
$\mathrm{PVF}=0.4$ & 1 & 1.5 & 2.5 & 0.4 & 3.24 \\
$\mathrm{PVF}=0.25$ & 1 & 3 & 4 & 0.25 & 4.75 \\
$\mathrm{PVF}=0.2$ & 1 & 4 & 5 & 0.2 & 5.58 \\
\hline
\end{tabular}

\subsection{Impact response of transparent plastics}

The contribution of each material constituent in bi-layered hybrids to impact response is evaluated in detail. The resultant effect of synergism to dissipate the incident impact energy is analysed through contact force $(\mathrm{F})$ and absorbed energy $\left(E_{a}\right)$ distribution curves. The extracted curves are manifested in terms of side of the impact i.e. soft and hard in order to observe the critical role of impact face. Table 4.2 lists the summary of basic properties of acrylic and PC materials required for the analysis.

Table 4.2: Basic mechanical properties of acrylic and polycarbonate (PC) sheets

\begin{tabular}{lllllll}
\hline Material & $\begin{array}{l}\text { Density } \\
\left(\mathrm{kg} / \mathrm{m}^{3}\right)\end{array}$ & $\begin{array}{l}\text { Young's } \\
\text { Modulus } \\
(\mathrm{GPa})\end{array}$ & $\begin{array}{l}\text { Tensile } \\
\text { strength } \\
(\mathrm{MPa})\end{array}$ & $\begin{array}{l}\text { Flexural } \\
\text { strength } \\
(\mathrm{MPa})\end{array}$ & $\begin{array}{l}\text { Failure } \\
\text { strain } \\
(\%)\end{array}$ & $\begin{array}{l}\text { Impact } \\
\text { strength } \\
\left(\mathrm{kJ} / \mathrm{m}^{2}\right)\end{array}$ \\
\hline Acrylic* & 1190 & 3 & 75 & 100 & 3 & 15 \\
PC $^{*}$ & 1200 & 2.4 & 60 & 90 & 110 & 50 \\
\hline
\end{tabular}

From supplier

\subsubsection{Contact force Vs impactor displacement}

At the instant when the impactor contacts the target, it was acknowledged that the initial elastic response of heterogeneous stacked material was primarily driven by the property of the layer which faces the impactor [6]. In the present case, having 
similar Young's Modulus for acrylic and PC laminates as listed in Table 4.2, the above hypothesis had been proclaimed where the initial slope of force-displacement curve attained by both the hard and soft face impact samples in Figure. 4.2 were apparently similar. Beyond the critical load, the abrupt drop significantly explores the influence of stacking sequence of bi-laminate hybrid configuration where the soft ductile side impact samples experience a sudden drop in contact force quite earlier than hard brittle side impact samples. This was anticipated due to inferior fracture toughness and smaller strain to failure of acrylic which accounts only around $3 \%$ (as listed in Table 4.2).

One might expect the flexural resistance of soft side impact target is to be improved by increasing the thickness of acrylic layer (i.e lowers the PVF). This is apparently not the case as observed in Figure. 4.2 which shows the force-deflection curves for different PVF samples. With increase in acrylic thickness and so the overall thickness, the contact force exhibited at the instant of abrupt loss in stiffness increases but the indentor displacement at this instant is always $2 \mathrm{~mm}$.

(a)

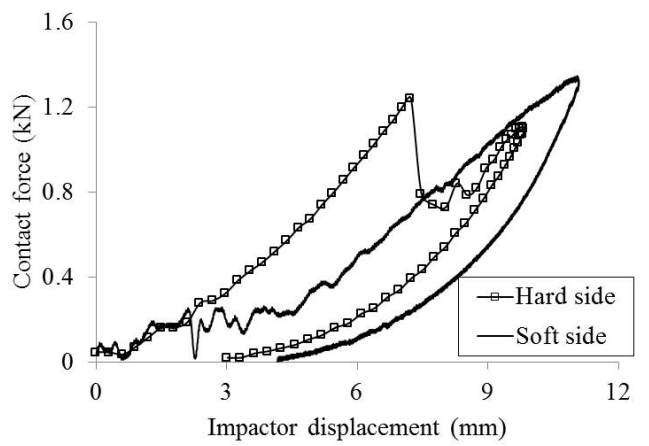

(c)

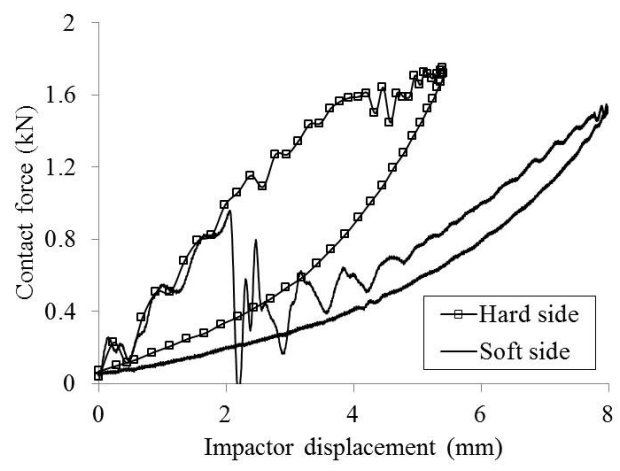

(b)

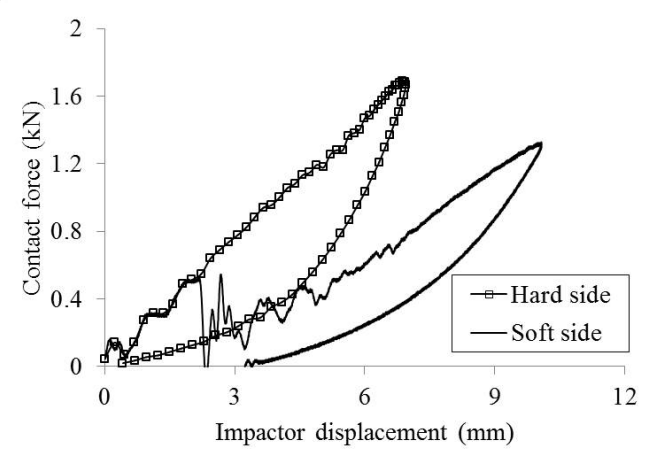

(d)

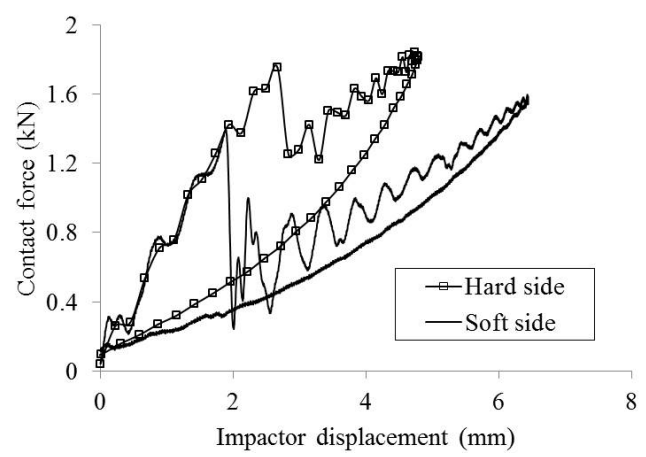

Figure 4.2: Force- deflection curve of PMMA-PC plastic laminates; (a) $\mathrm{PVF}=0.5$ (b) $\mathrm{PVF}=0.4$ (c) $\mathrm{PVF}=0.25$ (d) $\mathrm{PVF}=0.2$ 
Regarding the hard side impact response, the structural integrity is conserved over larger range of deformation where the degradation in stiffness is delayed for all PVF. Incorporating a ductile PC layer on the back side can provide an adequate elastic foundation for brittle acrylic layer who receives the impactor first with notable flexure. This combination delays the formation of radial cracks and also increases the contact load resistance

The magnitude of peak contact load dictates the level of impact resistance offered by the respective target samples. In the present case of soft side impact, except PVF 0.5, it can be seen the maximum contact force obtained by the other PVF samples are lower than its counterpart. Reasons relevant to the sole contradictory behaviour of PVF 0.5 are briefly discussed with the help of impact damage patterns in Section 4.4 .

\subsubsection{Absorbed energy Vs impactor displacement}

Figure. 4.3 portrays the energy-displacement trends of experimented samples of different PVF in terms of impact direction. Noted that none of the sample is perforated, so all the incident impact energy has to be consumed by the target via elastic, plastic deformation and by generating new damage surface. At the moment when the impactor transforms all of its kinetic energy, the spring back effect of target expels out the elastically stored internal strain energy. Thus, the energy absorption curves were incremented from 0 to maximum impact energy $(\sim 6 \mathrm{~J})$ and then restore part of them during unloading as illustrated in Figure. 4.3 .

As anticipated, the initial energy absorption trend of soft and hard side samples follows similar response due to similar Young's Modulus values. Following that, the critical role of impact direction was apparently emerged, after the moment when soft side sample loses its bending stiffness due to tensile cracking of back face brittle layer. It turns out that the energy absorption ability and the rate of energy absorption magnitude per impactor displacement was significantly decreased. On close scrutiny, the amount of energy necessary to induce such serious degradation was only about 0.3-1.5 J i.e. approximately 5-25\% of total impact energy. This indicates the level 
(a)

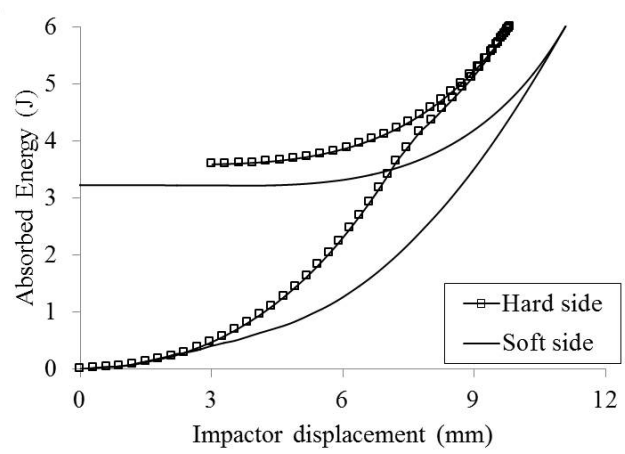

(c)

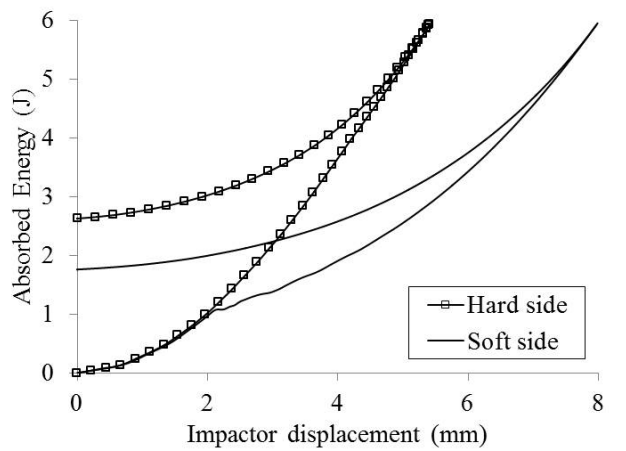

(b)

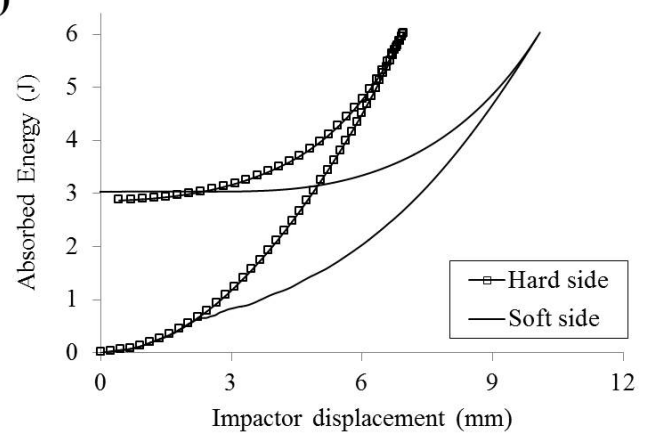

(d)

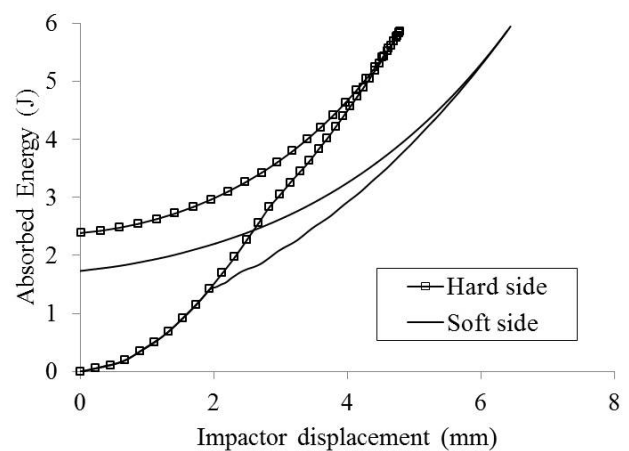

Figure 4.3: Energy- deflection curve of PMMA-PC plastic laminates; (a) PVF=0.5 (b) $\mathrm{PVF}=0.4$ (c) $\mathrm{PVF}=0.25$ (d) $\mathrm{PVF}=0.2$

of energy required to initiate and propagate a damage surface in acrylic layer is very low. The probable reasons for these sudden damage initiation and very large damage extension are due to inferior fracture toughness and higher crack propagation speed respectively. For a thin brittle plate, the crack propagation speed was found equivalent to the speed of elastic stress waves and it was given by a relation [132];

$$
\dot{a}=\frac{0.696+0.896 \nu}{1+\nu} \sqrt{\frac{E}{2 \rho(1+\nu}}
$$

Based on the the mechanical properties listed in Table 4.2, the crack propagation speed measures around $800 \mathrm{~m} / \mathrm{s}$. For the present scenario, the calculated speed consume only $47 \mu \mathrm{s}$ to cover the maximum target plate radius of $38 \mathrm{~mm}$ which is negligibly small when compared to recorded average contact duration of $13 \mathrm{~ms}$. Thus, the sudden high velocity fracture of acrylic layer consumes only negligible fraction of total impact energy and the remaining 80-90\% has been dissipated by the ductile PC layer either in the form of denting (localized indentation) or elastic dishing (flexural and stretching deformation) or by both happening in and around 
the impact region respectively.

Under comparable impact conditions, impactor pushes the soft side impact samples more heavily because of earlier loss in flexural rigidity, and thus covers larger displacement than hard side facing samples. Nevertheless, as confirmed from Figure. 4.3 the excessive deformation in soft side impact samples is attributed mostly by elastic deformation because most part of its absorbed energy had been restored back to impactor than hard side impact samples. This scenario dictates the flexural ability and the plastic deformation of interply hybrid laminates were significantly disturbed by the nature of the loaded surface. Especially for two ply hybrids, placing hard rigid plies on the back face had the tendency to inhibit the plastic deformation of tough soft plies 6 .

\subsection{Damage morphology of transparent plastics}

In the context of normal Hertzian contact mechanics, the stress fields that cause damage on impact event includes large component of hydrostatic compressive stresses on the impact face and modest tensile stress field on the non-impact back face. For layered systems consisting of different material types, the mismatch in material properties and the prevalence of considerable shear stress components would alter the damage pattern and the final morphology of fracture surface becomes quite complex.

The dynamic fracture of brittle blocks under impact loading is generally characterized by complex cracking surface having radial, conical, circumferential, median and lateral form of cracks [125]. In case of firmly bonded bi-layered plate made of hard and soft layers, along with the aforementioned damage patterns, the resultant damage surface is either incomplete penetration along with interfacial fracture or on the other hand complete perforation with severe form of damage like spalling, plugging and petalling if the impact kinetic energy is enormous [131 as illustrated in Figure 4.4 .

In all four experimented PVF, the damage patterns of targets having soft layer 


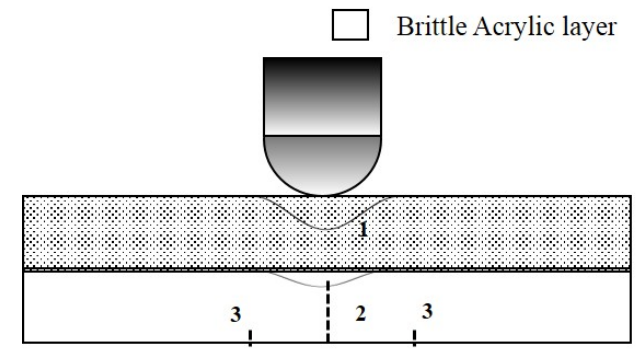

(a) Soft side impact
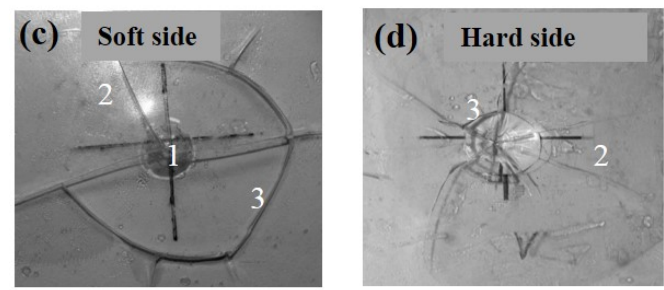

$\mathrm{PVF}=0.5$

1. Indentation dent of front face

2. Radial crack

3. circumferential crack (Spalling)
Ductile Polycarbonate layer

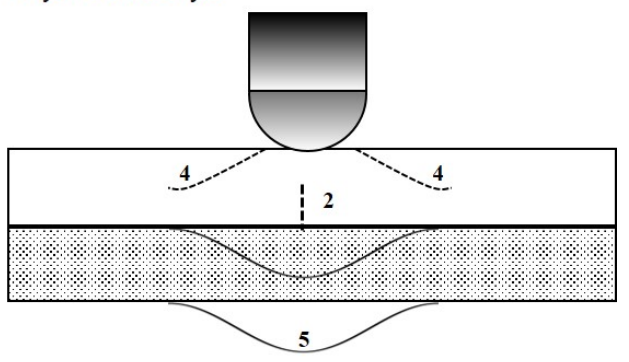

(b) Hard side impact
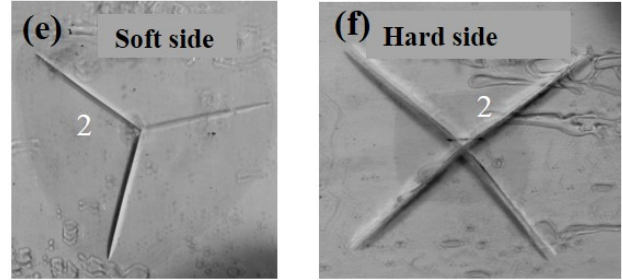

$\mathrm{PVF}=0.2$

4. Hertzian cone crack

5. Local flexure of back face

Figure 4.4: Damage patterns of impacted PMMA/PC plastic laminates

on impact face contain notable indentation dent of PC layer under the impact region along with either complete spalling or contours of radially propagated tensile cracks on the brittle back face acrylic layer. Spalling failure is commonly present in thin brittle layers as shown in Figure. 4.4(c) where a series of concentric transverse cracks are generated around the contact region due to notable mismatch in flexural behavior. Such tendency of spalling fracture was predominantly hindered by increasing the thickness of acrylic layer as depicted in Figure. 4.4(e) where radial crack is the major state of failure. But, one should note that both of these failure modes can degrade the stiffness of the laminate and no variation were existed in its characteristic response as shown in Figure 4.2 and 4.3

Likewise on impacting hard face, specimen with thin PMMA layers (PVF 0.5) shows a piece of fragments immediately under the center of impact and was presumably caused by multitude of tiny micro cracks nucleated around the contact area of target panel due to severe compressive contact pressure acting over it as shown in Figure 4.4(d). These devastating localized failures made the hard face impact sam- 
ple as inferior than the equivalent soft side impact sample which results a unique contradict response compared to other PVF hard side impact cases. Meanwhile, as the thickness of the PMMA layer is increased, the brittle layers are strong enough to withstand the contact pressure and the fracture surface contains only star shaped cracks of nearly 3 to 5 fragments propagated radially outwards to the boundary as illustrated in Figure 4.4(f). Furthermore,close investigation reveals that the length and the intensity of crack depth is remarkably increased with a decrease in PVF (or PMMA thickness) and this difference in crack geometry reflects measurable increase of drop in the contact force values of hard side impact samples as in Figure 4.2.

In addition, there is an optimum brittle layer thickness (here, it is PVF 0.4) in which there is no significant drop in contact force prevails and the curve resembles smooth distribution. Thus in order for optimum tolerance, the thickness of brittle layer laid with the ductile layer should be neither thin nor thick but should be intermediate enough to withstand the contact pressure and able to flexure well enough. The deformation mechanism of such optimum target panels are further analyzed via the in-plane and transversely bisected damage patterns.

(a)

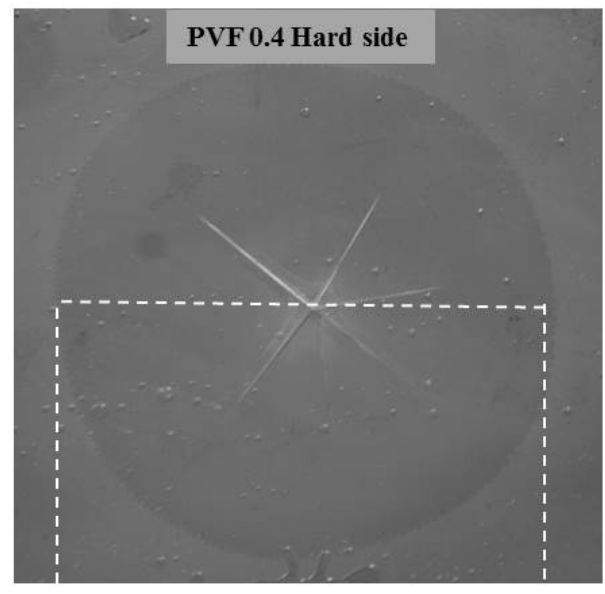

(b)

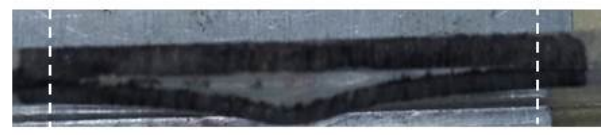

Figure 4.5: Cross sectional photography showing delamination of PVF 0.4 hard side impact sample

Having a ductile PC layer as an elastic foundation, a pattern of radially symmetric cylindrical zone was observed in the interlaminar region which is believed 
to be a shear dislocation of adhesive interface. This is in agreement with earlier findings of Tsai and Kolsky 126] in their classical work of Hertzian impact between steel ball and glass block in elastic half space. A radially propagated symmetrical wave with a cylindrical wave front called Rayleigh surface wave was captured over the glass surface on the impact center during the contact event. The disturbance associated with such wave front was found greatly decayed with respect to depth of glass block [126]. In the current study, the locus of circular dislocation zone (on Figure 4.5(a) was apparent on the interface region and the locus was decreased with increase in thickness of upper impact face layer (refer Figure 4.4(e) and 4.4(f) where the interface layer in former lies very near to impact surface and the disturbance bound is extended larger than the latter where the interface layer lies much below because of thicker acrylic layer). Furthermore, sectioning the impacted samples show that dislocation of interface zone due to Rayleigh stress waves eventually leads to de-bonding or delamination like the one shown in Figure. 4.5(b).

\subsection{Summary of conclusion}

The main conclusions from the LVI experiments on transparent plastics are presented herein.

- With no backing layer, the fractured region of brittle acrylic layer finally ensued to spalling fracture or severe radial crack propagation on soft side impact samples. This argument showed that after an earlier abrupt drop, the rest of the impact resistance in soft side impact sample was dependent on ductile PC layer.

- The impactor displacement at which the abrupt drop happens in contact force was almost constant for all cases of PVF. This behaviour highlighted that damage tolerance of hybrid panel was not enhanced whatever the thickness of brittle layer on soft side impact panels.

- The extent of delamination was found proportional to the position of interface region from the impact surface. With increase in acrylic thickness, the 
delamination envelopes of hard face impact samples were steadily decreased.

- Impact characteristics of hard face impact sample shows strong dependence on acrylic thickness. Having the layer of intermediately thick acrylic (PVF 0.4) provide optimum impact performance whose contact force curve resembles smooth and kink free that signifies the deformation and damage dissipation mechanism of the hybrid panel are in control throughout the impact event.

The implemented cost effective similitude LVI tests using transparent heterogeneous bi-layers provide a valuable information about internal damage surfaces and it allow to evaluate the contribution of individual material constituent to impact load with respect to initial impact surface. Besides, these conclusions provide a confidence to pursue similar approach on FML in order to extract the role of individual material constituents enclosed with it. 


\section{Chapter 5}

\section{Role of material constituents in FML}

This chapter presents the quasi-static indentation (QSI) and low velocity impact (LVI) behaviour of two FML configurations: FML-1/1 and FML-2/1. Details of material fabrication and test configurations are given in Section 5.2. Impact experiments on transparent interply hybrid plastics presented in Chapter 4 concluded that impact behaviour of bi-layered hybrid is dependent on whether the impact face is soft or hard. Influence of initial contact surface in FML is studied through $1 / 1$ configuration in Section 5.3. Following that, Section 5.4 extends the study to investigate the impact behaviour of FML-2/1 panel. These series of characterization studies provide a fundamental understanding of each material constituents and estimate their individual role in FML subjected to low velocity loading.

\subsection{Introduction}

FML is the heterogeneous interply hybrid of two distinct material characteristics systems namely; a ductile metal layer that consumes most of the energy as plastic work before fracture and a brittle composite layer that expends most of the energy in the form of different damage mechanisms with negligible plasticity. This contrary energy dissipation behaviour of these material systems must be known in detail for all sorts of mechanical loading prior to any structural application. In the present work, it is of interest to understand its role on indentation and impact loading.

Several experimental studies have been found in the literature that attempted to 
evaluate the influence of material constituents of interply hybrid composites containing brittle and ductile fibres 134 137]. Altogether, stacking clustered asymmetric fashion with brittle layer on the impact side backed by ductile layer was perceived as an optimal layup configuration for better impact response [134,137]. However, such detailed layer wise behavioural study has not yet been performed for FMLs.

\subsection{Test structure}

\subsubsection{Materials and Fabrication procedure}

The layers of hybrid laminates were made from AL-2024-O Clad aluminium sheets of $0.5 \mathrm{~mm}$ thickness and 7781/L-530 plain weave glass/epoxy composite prepreg of $0.254 \mathrm{~mm}$ thickness. Both metal and prepreg layers were trimmed to $100 \mathrm{~mm} \mathrm{x}$ $100 \mathrm{~mm}$ dimensions and stacked appropriately over a base plate. Before stacking, metal layers were subjected to proper surface treatment using sandpaper and surface cleaning using acetone. The stacked hybrid plates were cured in an autoclave at a constant $0.3 \mathrm{MPa}$ pressure with a maximum of $120^{\circ} \mathrm{C}$ temperature for $150 \mathrm{~min}$.

As can be seen in Table 5.1, three different kind of FML panels were fabricated: (i) Pseudo-bonded sample - no physical bond between metal and composite layer (no bond), GFRP layers were cured separately and stacked with metal layer during the test, (ii) Self-bonded sample - physical bond generated through self adhesive (epoxy) synthesized from GFRP prepreg layer during the curing process, (iii) Bonded sample - enhanced physical bond strength through introducing Redux (RE) 335K-300gsm adhesive film between GFRP prepreg and metal layer during stacking.

Table 5.1: Stacking configuration of FML panels used in indentation and impact tests

\begin{tabular}{lll}
\hline \multirow{2}{*}{ Nomenclature } & \multicolumn{2}{c}{ Stacking configuration } \\
\cline { 2 - 3 } & \multicolumn{1}{c}{ FML-1/1 } & \multicolumn{1}{c}{ FML-2/1 } \\
\hline Pseudo & $\mathrm{AL} /$ / GFRP & $\mathrm{AL} / / \mathrm{GFRP} / / \mathrm{AL}$ \\
Self & $\mathrm{AL} / \mathrm{GFRP}$ & $\mathrm{AL} / \mathrm{GFRP} / \mathrm{AL}$ \\
Bond & $\mathrm{AL} / \mathrm{RE} / \mathrm{GFRP}$ & $\mathrm{AL} / \mathrm{RE} / \mathrm{GFRP} / \mathrm{RE} / \mathrm{AL}$ \\
\hline
\end{tabular}




\subsubsection{Test configurations}

Indentation and single impact tests of different impact velocity $\left(V_{i}\right)$ was performed to characterise the damage and perforation threshold of FML panels. Figure 5.1 illustrates the flowchart of tests performed to predict the role of material constituents of FML. Asymmetric FML-1/1 laminates were fabricated by bonding single layer of metal sheet with layers of woven glass epoxy prepreg. As a benchmark study, QSI and LVI tests were performed on FML-1/1 panel of two distinct contact surface designated as soft side and hard side as illustrated in Figures 5.2 (a) and 5.2 (b). These cases will assess the role of elastic-plastic metal and elastic composite layer for two different deformation states. To estimate the role of adhesive interface between metal and composite in FML, panel having two extreme MCI surface i.e. self and pseudo bonded samples were tested and compared.

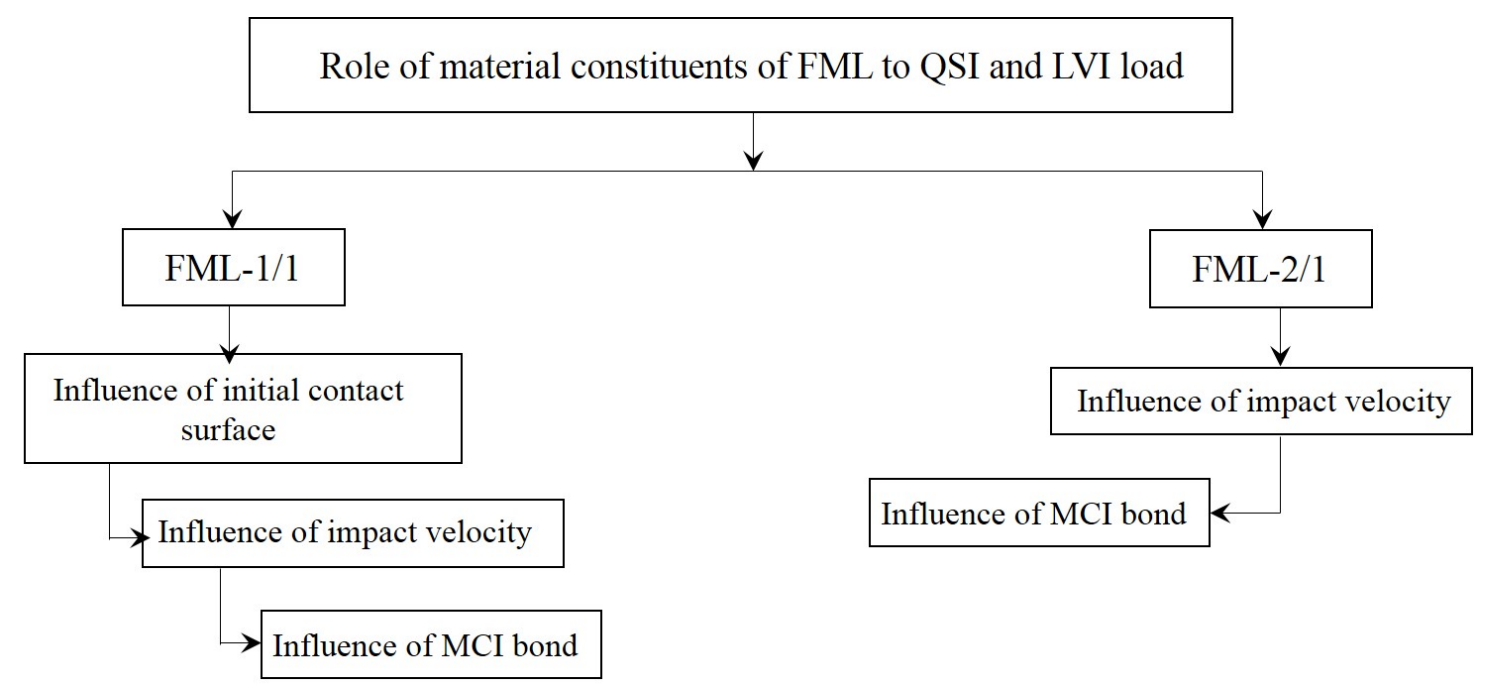

Figure 5.1: Flowchart of test carried to understand FML behaviour

With a thorough understanding about the role of material constituents in FML1/1 configuration, similar investigations were extended to FML 2/1 panel consisting a composite layer covered by metal sheets on both side as depicted in Figure 5.2 (c). Furthermore, different ways of energy absorbing characteristics of stacked layers were quantitatively compartmentalized with the help of reliable numerical simulations. 


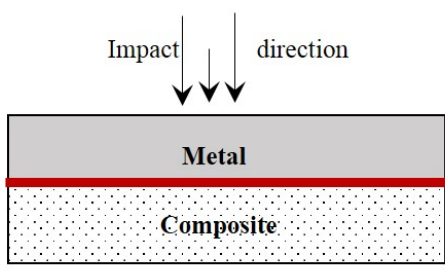

(a)

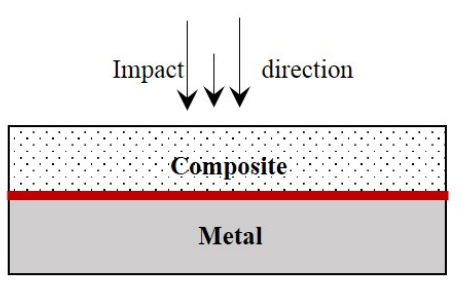

(b)

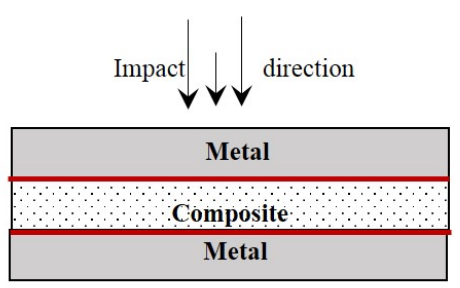

(c)

Figure 5.2: Different configuration of FML panel (a) FML-1/1-Soft side (b) FML1/1-Hard side and (c) FML-2/1

\subsection{Indentation and impact behaviour of FML- $1 / 1$ panel}

\subsubsection{Influence of initial contact surface}

On the onset of contact between impactor and target, the incident kinetic energy transferred to the target plate can be dissipated in various forms depending on the corresponding mechanical property of initial contact surface. For instance in the case of FML-1/1 panel, initial contact on ductile metal (soft) surface effectively dissipates the incident energy more locally by developing local indentation whilst initial contact on brittle composite (hard) surface actually distributes the incident energy through global flexure energy over larger area. Moreover, preliminary investigations carried on transparent brittle/ductile hybrid plastic in Chapter 4 confirmed the stated hypothesis. Thus, this study can help to understand the role of individual material layers of FML for the two extreme deformation behaviours.

Details of the geometry and stacking configurations of the test panels used in the investigation are given in Table 5.2. In order to assess absorbed energy difference between different surface contact samples, non-perforated impact damage is of interest here. Using Up-and-Down approach as described in ASTM D5420 [89], an appropriate impact energy level required to induce non-perforated damage for the given thick hybrid panel was estimated to be $4 \mathrm{~J}$ - 7J. As such, an impact energy of $6 \mathrm{~J}$ was chosen in this study. 
Table 5.2: Configurations used to study influence of initial contact surface of FML$1 / 1$ panel

\begin{tabular}{|c|c|c|c|c|c|c|c|}
\hline \multirow{2}{*}{ Sample } & \multirow{2}{*}{ Catergory } & \multirow{2}{*}{ Nomenclature } & \multirow{2}{*}{ Stacking sequence } & \multicolumn{3}{|c|}{ Thickness [mm] } & \multirow{2}{*}{ MVF } \\
\hline & & & & $t_{\text {metal }}$ & $t_{\text {comp }}$ & tlam & \\
\hline \multirow{4}{*}{ FML-1/1 } & \multirow{4}{*}{ Bond } & Soft & $\mathrm{M} / \mathrm{A} / \mathrm{C} / \mathrm{C}$ & 0.5 & 0.5 & 1 & 0.5 \\
\hline & & Soft & $\mathrm{M} / \mathrm{A} / \mathrm{C} / \mathrm{C} / \mathrm{C}$ & 0.5 & 0.75 & 1.25 & 0.4 \\
\hline & & Hard & $\mathrm{C} / \mathrm{C} / \mathrm{A} / \mathrm{M}$ & 0.5 & 0.5 & 1 & 0.5 \\
\hline & & Hard & $\mathrm{C} / \mathrm{C} / \mathrm{C} / \mathrm{A} / \mathrm{M}$ & 0.5 & 0.75 & 1.25 & 0.4 \\
\hline
\end{tabular}

Note: $\mathrm{M}, \mathrm{A}$ and $\mathrm{C}$ represents $\mathrm{AL}, \mathrm{RE}$ and GFRP respectively.

\subsubsection{Observed experimental response}

Figures 5.3(a) and 5.3(c) shows the impact damage surfaces of soft side contact samples of $\mathrm{MVF}=0.5$ and $\mathrm{MVF}=0.4$ respectively. The compressive contact stress generated close to the point of impact causes severe local plastic deformation on front aluminium surface that resulted a visible permanent dent. Moreover, this severe local deformation induced a tensile crack surface on the GFRP layer. Having axisymmetric boundary conditions and possessing balanced amount of fibres along weft and warp directions, the crack on woven GFRP layer extended as symmetric and in mutually perpendicular directions about the impact centre.

$\underline{\text { MVF- } 0.5}$
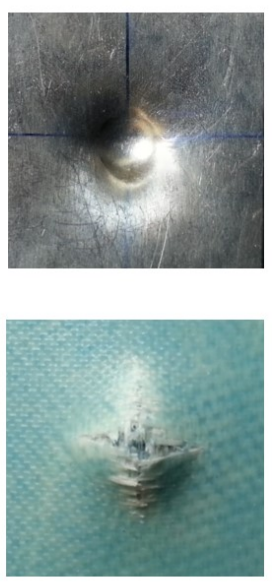

(a)
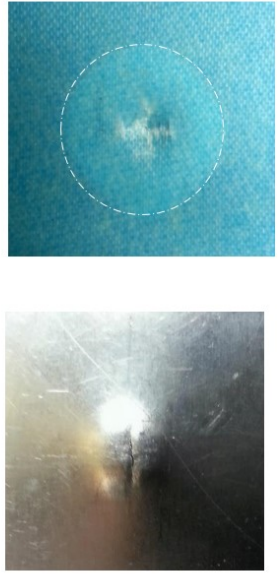

(b)

MVF- 0.4
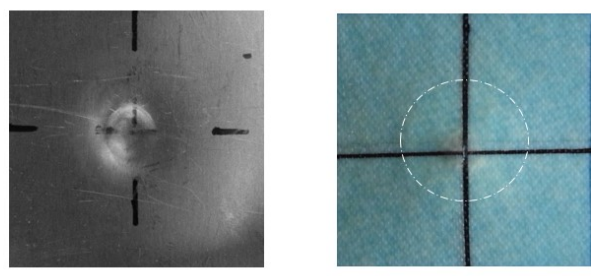

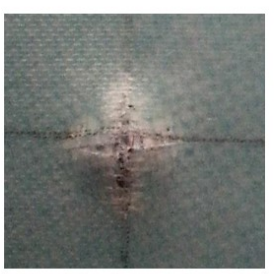

(c)

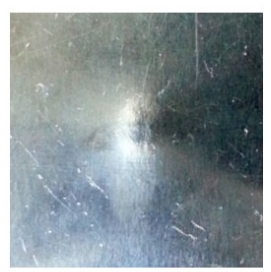

(d)

Figure 5.3: Impact damage surfaces of FML-1/1 panel having different MVF: MVF 0.5 (a) Soft side (b) Hard side and MVF 0.4 (c) Soft side (d) Hard side

The lack of plastic flow of GFRP layer leaves no evidence of indentation dent on hard surface contact sample as can be seen in Figure $5.3(\mathrm{~b})$ and $5.3(\mathrm{~d})$. The contact stresses crushes the glass fibres present underneath the contact region and 
create small tiny cracks on the front GFRP layer, and the bottom aluminium layer underwent noticeable plastic deformation. A small thin crack was observed at the impact center for MVF=0.5 FML panel.

The locus of Metal Composite Interface (MCI) de-bond boundary is obvious through transparent GFRP layer in the form of extended whitening region. Observations show no wide spread MCI de-bond on soft side contact sample while de-bond boundary spread over larger area than the maximum contact region on hard side contact sample.

Characteristic curves in terms of load versus deflection and absorbed energy versus deflection curve are shown in Figure 5.4(a). This figure provides multiple information such as initial contact stiffness (initial slope of the load versus displacement curve), threshold force $\left(P_{t h}\right)$ (instant at which first drop in contact force due to damage initiation in target panel), maximum contact force $\left(P_{\max }\right)$, energy at threshold $\left(E_{t h}\right)$ and total absorbed energy $\left(E_{a b s}\right)$ on contact surface influence on different MVF FML-1/1 sample.

Despite the fact that laminates of both cases have equal overall flexural stiffness, the contact stiffness of soft side contact sample record larger force values than its counterpart until the threshold limit. This phenomenon confirms the hypothesis that the initial period of contact is primarily controlled by the layer that receives the impactor [6]. By analogy, soft side sample has higher elastically stiff aluminum layer $(\mathrm{E}=70 \mathrm{GPa})$ on the impact face and thus able to bear larger force than hard surface contact sample which has comparatively lower stiff GFRP layer $\left(E_{i}=23 \mathrm{GPa}\right.$, $\mathrm{i}=1,2)$.

Regardless of the value of MVF, the threshold limit of hard side contact sample $\left(P_{t h}\right)_{h a r d}$ is observed to be larger than that of soft side contact sample $\left(P_{t h}\right)_{s o f t}$. This is because, having ductile backing layer, hard side contact samples were able to bear larger contact load without initiating any damage over larger displacement. For panels impacted on soft side, the inferior tensile fracture strain of GFRP layer (4\%) resulted in earlier damage initiation. 


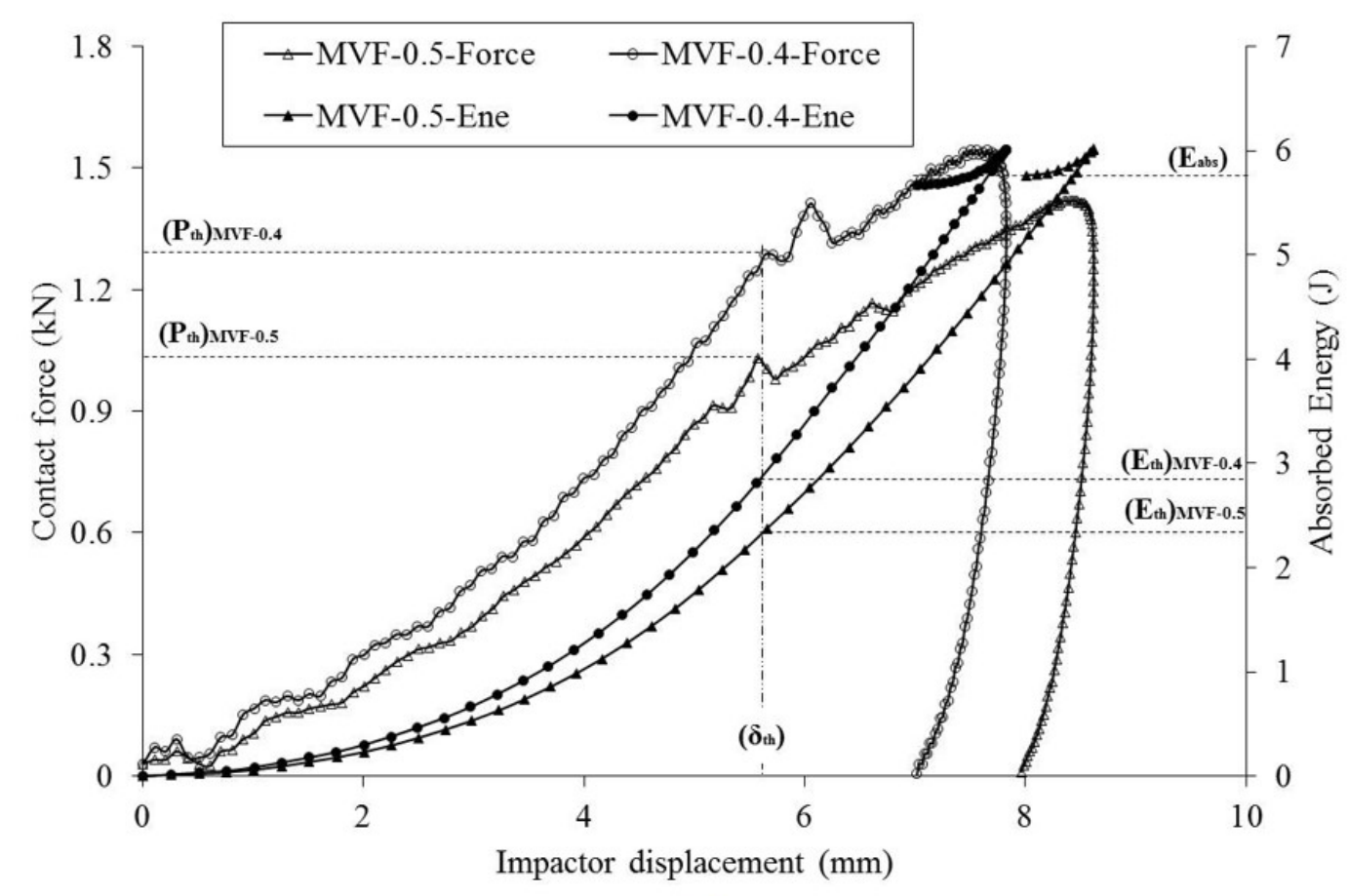

a)

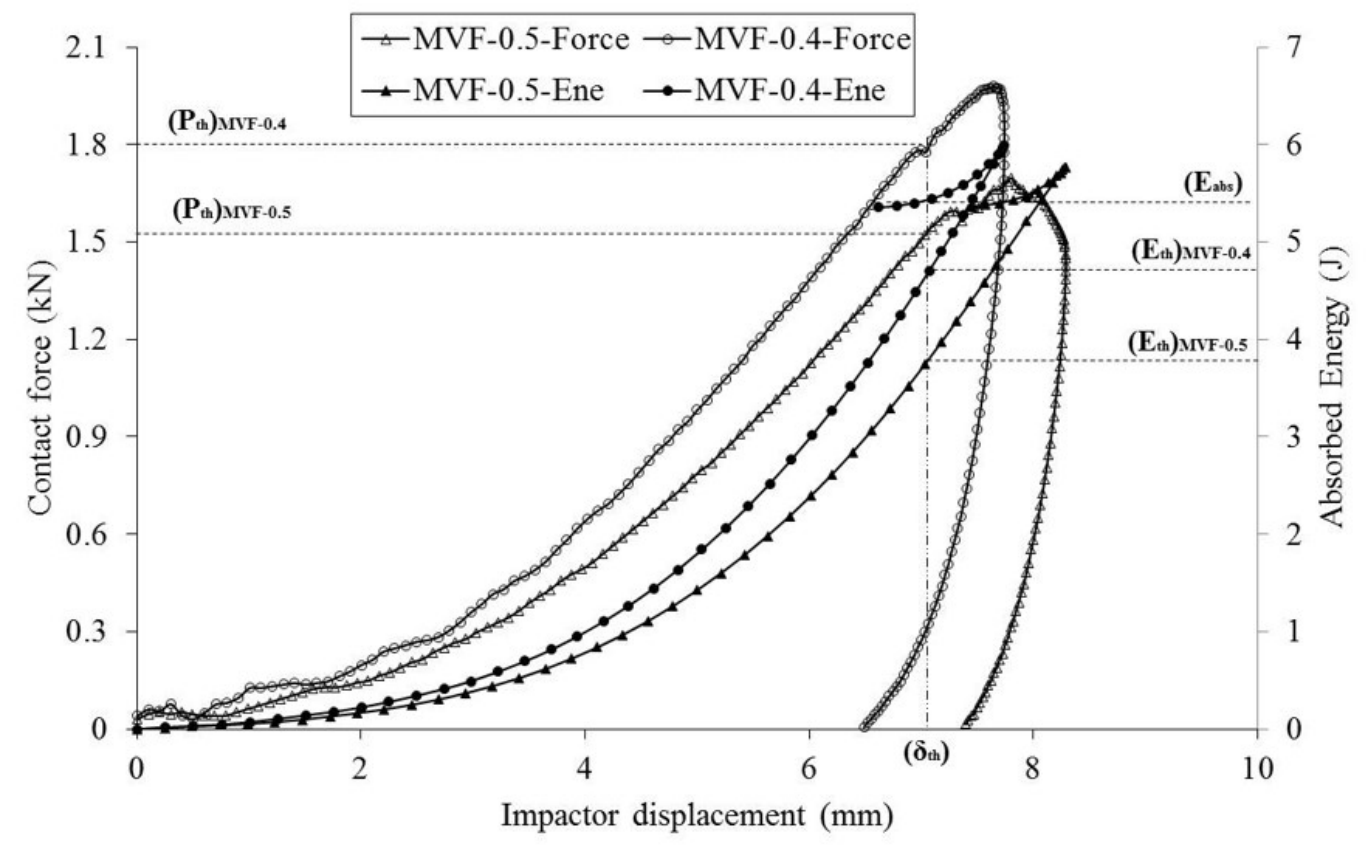

b)

Figure 5.4: Impact characteristic curves of FML-1/1 panel having different MVF (a) Soft side (b) Hard side 
With pronounced undamaged global flexural deformation, the overall stiffness of the sample continuously increases until the impactor dispersed all of its kinetic energy. As a result of enhanced resistance to local indentation of the front composite layer and extended hardening of the bottom aluminium layer, the peak contact force $\left(P_{\text {max }}\right)_{\text {hard }}$ of hard contact impact sample registers larger value than soft side contact sample $\left(P_{t h}\right)_{\text {soft }}$.

The threshold energy absorbed to nucleate new damage surface for hard contact sample $\left(E_{t h}\right)_{h a r d}$ is found to be nearly 1.5 - 2 times greater than that of soft contact sample $\left(E_{t h}\right)_{\text {soft }}$. However, the net energy absorbed by the same $\left(E_{a b s}\right)_{h a r d}$ was found to be smaller than its counterpart $\left(E_{a b s}\right)_{\text {soft }}$. This scenario raises a speculation that there exists a difference in relative percentage of energy absorption among different damage dissipation modes between soft and hard side contact samples. This notional conclusion is verified by means of reliable FE simulation as discussed in the following section.

\subsubsection{Predicted numerical response}

Figure 5.5 shows the finite element predictions of contact force history and damage morphology of experimental observation for $\mathrm{MVF}=0.5$ FML samples. The predictions are well correlated both in terms of qualitative and quantitative. This deduction also applies to the comparison of the results of $M V F=0.4$. Difference in the quantitative data between $\mathrm{MVF}=0.5$ and $\mathrm{MVF}=0.4$ are treated separately in subsequent section.

MCI de-bond failure in FML can be induced either by fibre/matrix failure of GFRP layer or by elastic mismatch between metal and composite layer [55, 60]. To replicate the de-bond damage, the cohesive elements used to model the MCI region are allowed to delete once it satisfies the damage evolution law. The FE predictions of MCI de-bond damage of FML-1/1 sample using cohesive elements is shown in Figure 5.5(c).

Similarly with experimental observations, the de-bond locus of soft side contact samples is found not extended beyond the fibre crack length of adjacent GFRP 


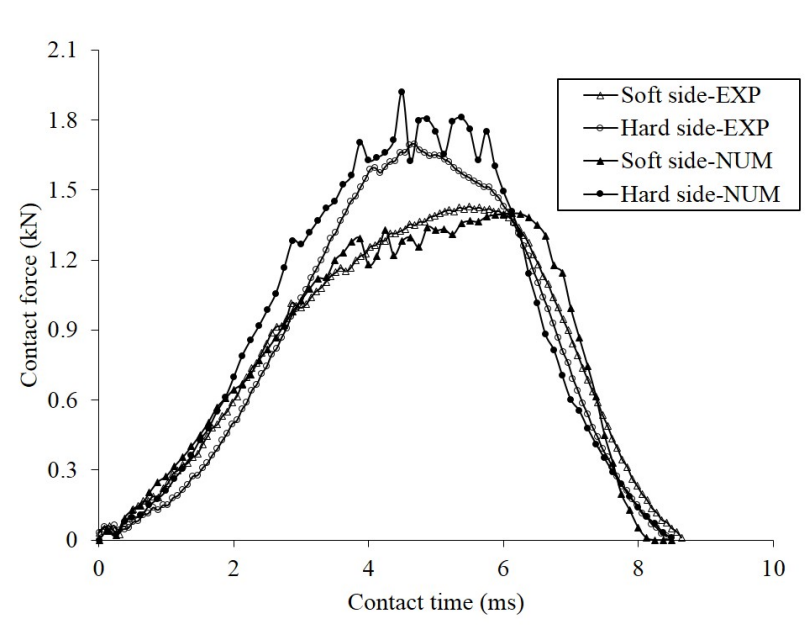

a)

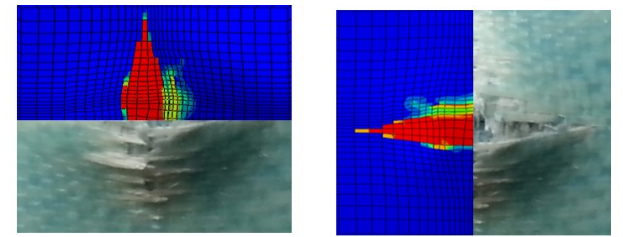

b)
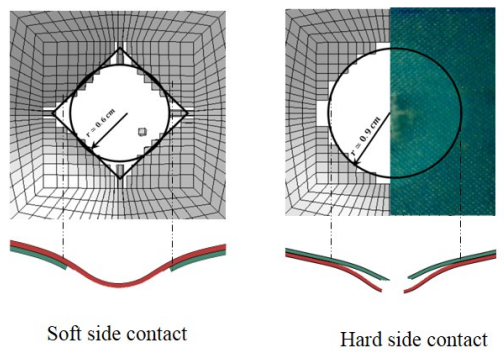

c)

Figure 5.5: Experimental and numerical impact response of FML-1/1-MVF=0.5 (a) Contact force history (b) Fibre damage of soft side impact panel and (c) MCI-debond shape

layer. The de-bond locus resembles rhombus in shape and its extent of damage is approximated by sketching an inscribed circle enclosing the sides of rhombus. In the case of hard side contact sample, the absence of confined local deformation allowed one to visually mark the de-bond locus through the transparent GFRP layer as depicted in Figure 5.3 and Figure 5.5(c). The observed de-bond boundary is circular in shape and its dimension $(\sim 9 \mathrm{~mm})$ measures greater than soft side contact sample $(\sim 6 \mathrm{~mm})$.

The cross sectional view of predicted impact damage in Figure 5.5(c) shows two distinct de-bond pattern along MCI depend on contact surface. In soft side contact sample, the spring back of elastic GFRP layer has been completely arrested by permanent plastic deformation of impact surface metal layer. As a result, the deformation profile of dissimilar material layers is in-phase with de-bond region extended until fibre crack zone. Meanwhile in hard side contact panels, the inhomogeneity in plastic deformation induce an apparent out of phase deformation where the bottom aluminium layer showed dome shaped plastic deformation but nothing on front impacted composite surface. These observations confirmed the speculation that the interfacial debonding in the former case was driven by fibre/matrix damage where as the inhomogeneity in plastic deformation is the primary cause in the latter case. 
In other words, the resistance to localized indentation of composite layer allowed greater surface area to share the contact stress on hard side contact sample which induce more shear stress along resin rich MCI region due to elastic mismatch. These cumulative phenomena eventually resulted in larger de-bond zone on hard side over soft side impact sample.

\subsubsection{Effect of MVF}

To study the influence of MVF of FML panel having different thickness, contact force and impactor displacements are expressed in non-dimensionalized form using the following relation:

$$
\begin{gathered}
\bar{P}=\frac{P}{E_{F M L} t R} \\
\bar{\delta}=\frac{\delta}{t}
\end{gathered}
$$

where $E_{F M L}=M V F E_{A L}+(1-M V F) E_{G F R P}, R$ is the impactor radius and $t$ is the laminate thickness, $\bar{P}$ and $\bar{\delta}$ represents non-dimensional quantities of contact force $(P)$ and impactor displacement $(\delta)$ respectively and $E_{i}$ is the Young's modulus of material ' $i$ '. Table 5.3 shows the magnitude of different characteristic parameters of FML-1/1 panel with different MVF contacted on soft and hard side surface

Table 5.3: Impact characteristics of FML-1/1 panel with different MVF

\begin{tabular}{lcc|cc}
\hline \multirow{2}{*}{ Parameter } & \multicolumn{2}{c|}{ Soft side } & \multicolumn{2}{c}{ Hard side } \\
\cline { 2 - 5 } & MVF 0.5 & MVF 0.4 & MVF 0.5 & MVF 0.4 \\
\hline $\bar{P}_{t h}\left(\mathrm{x} 10^{-3}\right)$ & 3.33 & 3.71 & 5.22 & 5.21 \\
$\bar{\delta}_{t h}$ & 5.167 & 4.626 & 7.364 & 5.635 \\
$\bar{P}_{\max }\left(\mathrm{x} 10^{-3}\right)$ & 4.71 & 4.54 & 5.6 & 5.81 \\
$\bar{\delta}_{\max }$ & 8.618 & 6.261 & 8.288 & 6.193 \\
$E_{s}\left(\mathrm{Jkg} / \mathrm{m}^{2}\right)$ & 2.561 & 2.308 & 2.385 & 2.177 \\
$t(\mathrm{~ms})$ & 8.643 & 8.203 & 8.439 & 8.023 \\
\hline
\end{tabular}

It can be noticed, with decrease in MVF, the threshold force $\left(\bar{P}_{t h}\right)$ of soft side contact samples increases, but remains constant for hard side contact samples. However, the impactor displacement at which the threshold occurs $\left(\bar{\delta}_{t h}\right)$ is earlier in both cases. Due to earlier failure, the peak load $\left(\bar{P}_{\max }\right)$ in lower MVF sample is smaller for soft side panels but contradictorily larger for hard side contact sample. This 
phenomenon is explained from the final damage shapes of impacted samples (see Figure 5.3). It depicted that, adding more composite layer (smaller MVF) does not improve the resistance to tensile cracking of GFRP layer in soft side contact panels but it protects the bottom aluminium layer from cracking in hard side contact samples.

Path line through impact center $\mathrm{X}-\mathrm{X}(\mathrm{mm})$

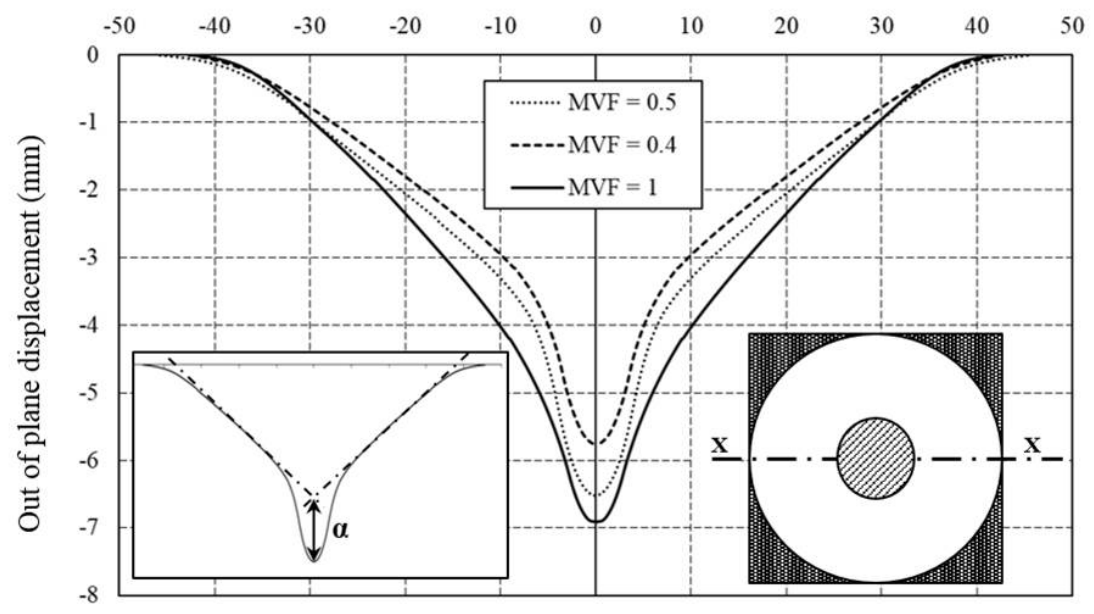

Figure 5.6: Deformation profile of soft side impacted FML-1/1 panel

The measured final permanent deformation shapes of experimented soft side impact samples shown in Figure 5.6 indicates that the slope of the bending profile and depth of local dent (local indentation ' $\alpha$ ') under the impact center decreases with decrease in MVF. As expected, the addition of more brittle composite layer increases the overall stiffness of the lower MVF panels and there by decreases the maximum impactor displacement $\left(\bar{\delta}_{\max }\right)$, specific energy (=energy/areal density) absorbed $\left(E_{s}\right)$ and contact time $(t)$ on both contact surface cases.

\subsubsection{Layer-wise energy partition}

Figure 5.7 illustrates the strain energy absorbed by each material layer of soft and hard surface contact samples. These layer wise predictions allow one to estimate the contribution level of each material layer on overall impact response. For better comparisons, the internal energy of each material layer in the ordinate of Figure 5.7 is normalized with their corresponding incident impact energy values. 


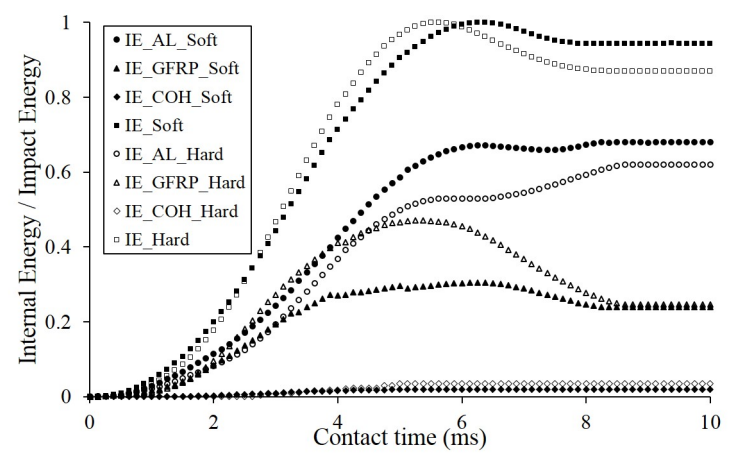

a)

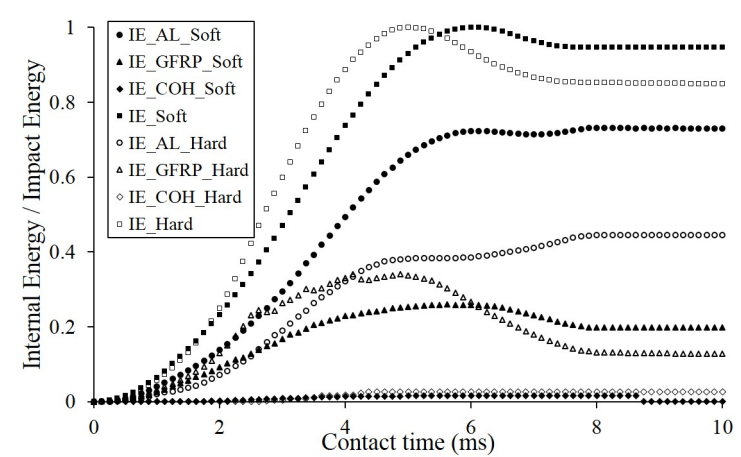

b)

Figure 5.7: Layer-wise energy partition of impacted FML-1/1 panel (a) MVF 0.5 (b) MVF 0.4

The key observations derived from the layer wise energy compartmentalization are:

(i) From the internal energy plots of the whole model (IE), it is obvious that hard side contact sample absorb less amount of energy than soft side impact. Material wise categorization showed that the reason for such variation is attributed mainly by the difference in energy absorption capability of aluminium layers (IE_AL) of panels impacted on different contact surface.

(ii) Regardless of the different values of MVF, energy absorbed by the metal layer (IE_AL) was found to be greater than the composite layers (IE_GFRP). This confirms that impact response of metal-layered hybrid samples is dominated mainly by the plastic flow in the metal layers.

(iii) Comparing the material wise internal energy quantity on each sample, the contribution of GFRP layer in soft side contact sample is found to be considerably overshadowed by that of the aluminium layer where substantial difference exists between the energy curves of corresponding material layers (IE_AL_Soft Vs IE_GFRP_Soft). However, such difference is almost negligible and show equal contribution from both material layers in hard surface contact sample (IE_AL_Hard Vs IE_GFRP_Hard). 


\subsubsection{Mode-wise energy partition}

The dissipation of impact energy by each material is primarily a function of its mechanical property. From the experimental study, it was observed that plastic deformation $(\mathrm{PD})$ of aluminium layer $(\mathrm{AL})$, tensile fracture of composite (GFRP) and de-bond damage of MCI $(\mathrm{COH})$ are the notable energy dissipation modes in FML panels. Besides that, some reasonable percentage of incident energy has also been generally accumulated in the form of recoverable elastic strain energy (EL) for the case of non-perforated impact event such as the present impact energy level. Thus, the above-predicted internal energy (IE) quantity of each material in Figure 5.7 can be further categorized in to respective energy dissipation modes of the form:

$$
E_{I E}=E_{I E}^{A L}+E_{I E}^{G F R P}+E_{I E}^{C O H}
$$

where $E_{I E}^{A L}=E_{P D}^{A L}+E_{E L}^{A L}+E_{D M D}^{A L}, E_{I E}^{G F R P}=E_{E L}^{G F R P}+E_{D M D}^{G F R P}$ and $E_{I E}^{C O H}=E_{D M D}^{C O H}$

On adding together,

$$
E_{I E}=E_{P D}^{A L}+E_{E L}+E_{D M D}
$$

where $E_{E L}=E_{E L}^{A L}+E_{E L}^{G F R P}$ and $E_{D M D}=E_{D M D}^{A L}+E_{D M D}^{G F R P}+E_{D M D}^{C O H}$.

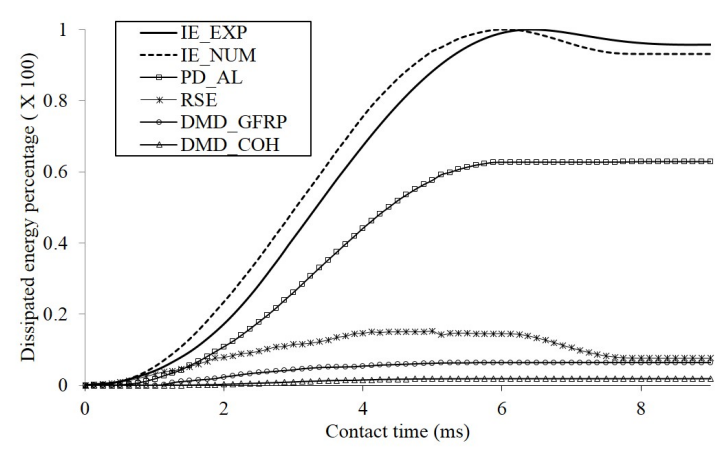

a)

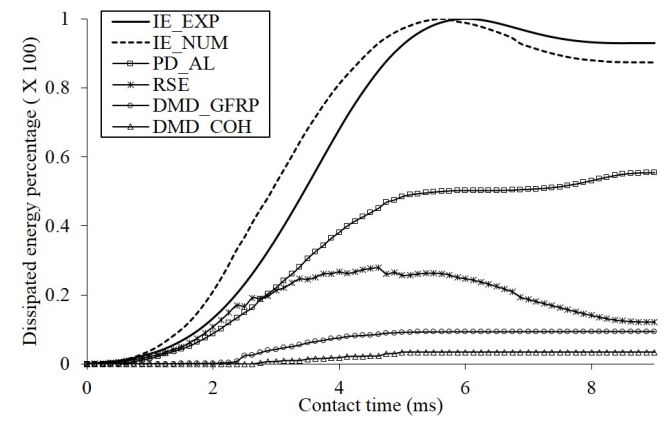

b)

Figure 5.8: Mode-wise energy partition of impacted FML-1/1- MVF 0.5 panel (a) Soft side (b) Hard side

Figures 5.8(a) and 5.8(b) illustrate the history of predicted mode-wise dissipated energy components of each material layer in $\mathrm{MVF}=0.5$ sample under soft and hard 
surface contact respectively. The distribution plot of $\mathrm{MVF}=0.4$ looks rather similar to $\mathrm{MVF}=0.5$ thus, only its quantitative data is summarized in Table 5.4 for comparison.

Table 5.4: Numerical predictions of different modes of energy dissipation

\begin{tabular}{lcc|cc}
\hline \multirow{2}{*}{ Parameter* } & \multicolumn{2}{c|}{ MVF 0.5 } & \multicolumn{2}{c}{ MVF 0.4 } \\
\cline { 2 - 5 } & Soft side & Hard side & Soft side & Hard side \\
\hline$E_{\text {abs }}$ & 0.93 & 0.87 & 0.92 & 0.85 \\
PD_AL & 0.63 & 0.55 & 0.56 & 0.45 \\
EL & 0.08 & 0.12 & 0.12 & 0.13 \\
DMD_GFRP & 0.06 & 0.09 & 0.09 & 0.11 \\
DMD_COH & 0.02 & 0.03 & 0.02 & 0.03 \\
\hline
\end{tabular}

${ }^{*}$ For better comparison, incident impact energy is taken as 1 .

In both cases, the fraction of energy dissipated by particular mode in FML-1/1 panel to impact load takes the following descending order; plastic deformation of aluminium layer, recoverable strain energy, intralaminar damage in composite layer and interfacial debonding along MCI. Comparison between the energy components of hard and soft surface contact sample provide the following key behavioural information:

(i) Having severe local indentation, metal layers of soft side contact samples consume more energy by means of plastic deformation (PD) than hard side contact samples.

(ii) Occurrence of composite and MCI de-bond damage on both soft and hard surface contact samples are simultaneous. However, their initiation is delayed for some time in hard surface contact sample.

(iii) Amount of energy absorbed by creating de-bond regions along MCI is found to be negligibly small compared to other energy dissipation modes (DMD_COH). This fact is expected since the fracture energy of adhesive resins are much smaller $(0.3-1.2 \mathrm{kN} / \mathrm{m})$ than fracture energy of polymer fibres $(20-50 \mathrm{kN} / \mathrm{m})$ and aluminium $(20 \mathrm{kN} / \mathrm{m})$.

(iv) With no constraints imposed by the metal layers, elastic recovery of GFRP 
layer (EL) in hard side contact samples is more pronounced than soft side contact samples (see Table 5.4).

\subsubsection{Influence of impact velocity}

This section attempts to study the influence of impact velocity on FML-1/1 panel having $\mathrm{MVF}=0.5$. Single impact test was conducted on soft and hard contact side with four different impact velocities that can produce damage from non-perforation to completely penetration. The main purpose of this study is to understand the influence of initial contact surface on the impact response over different damage intensity levels. Numerical simulations were also performed to extract damage history and estimated the dissipated energy through distinct modes. Table 5.5 summarizes the panel configuration and loading conditions used to study the impact velocity influence.

Table 5.5: Configurations used to study influence of initial contact surface of FML$1 / 1$ panel

\begin{tabular}{cccccc|c}
\hline \multirow{2}{*}{ Sample } & \multirow{2}{*}{ Catergory } & \multirow{2}{*}{ Nomenclature } & \multirow{2}{*}{ Stacking sequence } & \multirow{2}{*}{ MVF } & \multicolumn{2}{c}{ Test condition } \\
\cline { 7 - 7 } & & & & QSI & LVI \\
\hline \multirow{2}{*}{ FML-1/1 } & \multirow{2}{*}{ Bond } & Soft & M/A $/ \mathrm{C} / \mathrm{C}$ & 0.5 & \multirow{2}{*}{$1 \mathrm{~mm} / \mathrm{min}$} & $1 \mathrm{~m} / \mathrm{s}, 1.5 \mathrm{~m} / \mathrm{s}$ \\
& & Hard & C $/ \mathrm{C} / \mathrm{A} / \mathrm{M}$ & 0.5 & & $1.75 \mathrm{~m} / \mathrm{s}, 2 \mathrm{~m} / \mathrm{s}$ \\
\hline
\end{tabular}

Note: $\mathrm{M}, \mathrm{A}$ and $\mathrm{C}$ represents $\mathrm{AL}, \mathrm{RE}$ and GFRP respectively.

Before attempting to investigate the impact response at different impact velocity, response of FML-1/1 laminate to quasi-static indentation (QSI) load was assessed. This preliminary QSI test allows one to understand the overall behavioral characteristics such as initial undamaged elastic-plastic response, damaged non-perforated response and final perforated response of target material. Besides, it allows one to trace the instant and sequence of distinct damage modes nucleated in the constituent material systems and also provide quantitative information of the energy level required to induce particular damage mode.

\subsubsection{Quasi-static indentation response}

QSI response in terms of Contact force $(P)$ and energy absorbed $\left(E_{a}\right)$ with respect to indenter displacement $(\delta)$ for FML-1/1 samples having two different initial 
contact surface is shown in Figure 5.9(a). Based on visual observation and the obtained characteristic curves $\left(P-\delta \& E_{a}-\delta\right)$, QSI response of FML-1/1 laminate can be classified in to three stages:

(I) Non-penetrated (Undamaged elastic-plastic) stage that increases in contact force from zero to threshold force value $\left(P_{t h}\right)$ where damage initiates.

(II) Partially penetrated (Damaged plastic) stage which shows continuous increase in contact force to maximum $\left(P_{\max }\right)$ until complete through thickness damage.

(III) Perforated plastic stage which displays continuous decrease in contact force from maximum $\left(P_{\max }\right)$.

It is obvious from Figure 5.9 (a) that the initial QSI response of FML panel were governed by whether the surface in contact between impactor and target is hard or soft. Visual observation confirmed that the threshold condition in both the contact surface cases is attributed to the damage of GFRP layer. As mentioned earlier in Section 5.3.1.1, the inferior tensile fracture strain of GFRP allows the soft side contacted FML panel to initiate earlier GFRP layer failure. The failure is reflected in the characteristic response where the threshold limit of soft side contact samples is at a smaller indenter displacement than hard side contact samples and the corresponding force $\left(P_{t h}\right)_{s o f t}$ and energy magnitudes $\left(E_{t h}\right)_{s o f t}$ records lower magnitude than its counterparts $\left(P_{\text {th }}\right)_{\text {hard }} \&\left(E_{\text {th }}\right)_{\text {hard }}$.

Even though soft side contact sample lost the contribution from GFRP layer at an earlier stage, the panel was able to resist larger contact force $\left(P_{\max }\right)_{\text {soft }}$ and absorbed more energy $\left(E_{\max }\right)_{\text {soft }}$ than hard side contact sample $\left(P_{\max }\right)_{\text {hard }} \&\left(E_{\text {max }}\right)_{\text {hard }}$. This is because the earlier reduction in flexural rigidity due to premature failure of GFRP layer allowed the soft side contact sample to undergo larger plastic deformation by both flexure and membrane stretching before perforation and thereby achieving larger $\left(P_{\max }\right)_{\text {soft }}$ and $\left(E_{\max }\right)_{\text {soft }}$. However, the prolonged resistance to flexure resulted the hard side panel to penetrate with a smaller load magnitude $\left(P_{\max }\right)_{\text {hard }}$. Therefore, the experimental observation of QSI response shows that, facing the 


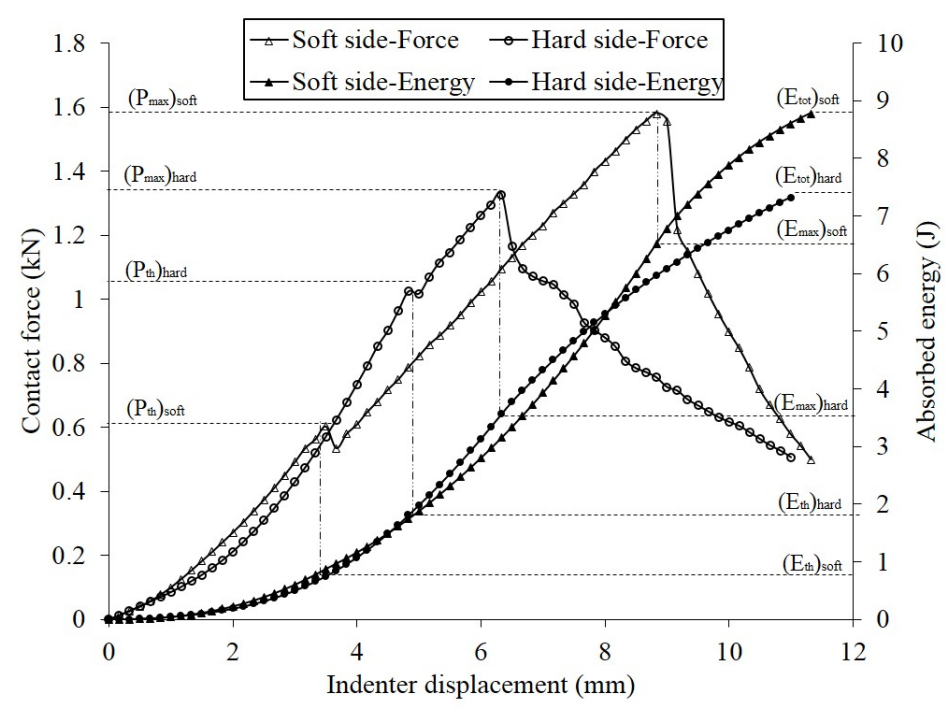

(a)

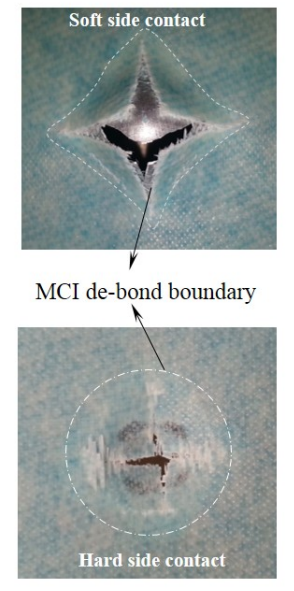

b)

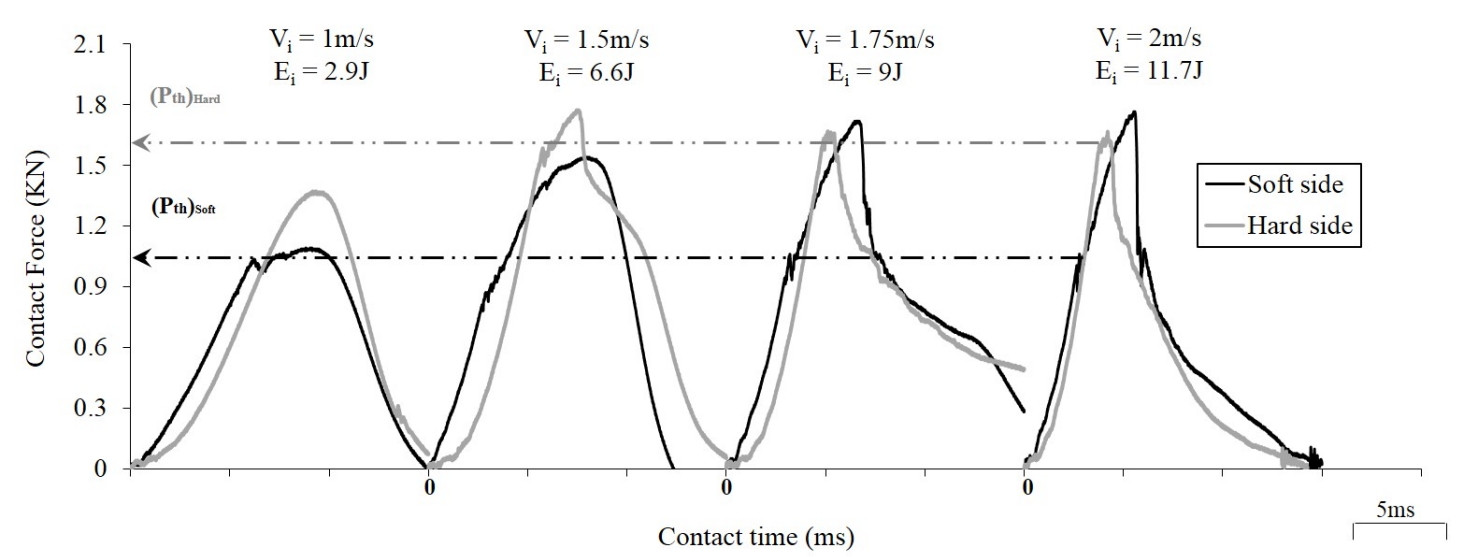

(c)

Figure 5.9: Indentation and impact response of soft and hard side impacted FML1/1 panel (a) Indentation characteristic curve (b) Indented damage shape and (c) Impact characteristic curve 
impact surface to hard side seems to be better for applications favoring damage resistance whilst placing soft impact surface seems to benefit applications that favored perforation resistance requirements.

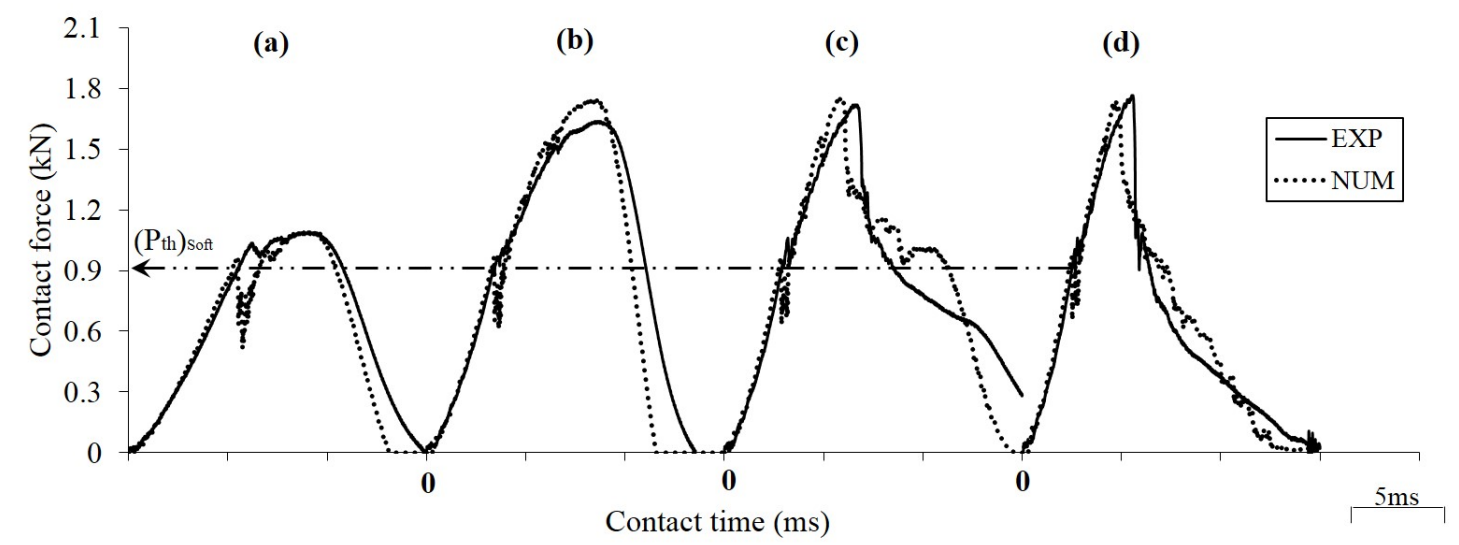

Figure 5.10: Experimental and numerical contact force histories of soft side impacted FML-1/1 panel (a) $V_{i}=1 \mathrm{~m} / \mathrm{s}$ (b) $V_{i}=1.5 \mathrm{~m} / \mathrm{s}(\mathrm{c}) V_{i}=1.75 \mathrm{~m} / \mathrm{s}$ and (d) $V_{i}=2 \mathrm{~m} / \mathrm{s}$

\subsubsection{Low velocity impact response}

Figure 5.9 (c) shows the contact force history for each impact velocity of hard and soft side impact sample. As can be observed, the influence of initial contact surface stiffness is significant for the range of velocity considered. Furthermore, the contact force magnitude at which the threshold condition $\left(P_{t h}\right)$ occurs remains constant regardless of impact velocity. And similar to the QSI test findings, the threshold force of soft side impact $\left(P_{t h}\right)_{\text {soft }}$ sample is lower than that of hard side impact $\left(P_{\text {th }}\right)_{\text {hard }}$ sample. Finite element predictions shown in Figures 5.10 and 5.11 confirmed the above conclusions of threshold conditions.

Final damage surface observed in experiment and numerical simulations, MCI debond region captured by the cohesive elements and cross sectional view of damaged panels under the contact region of soft and hard side impacted panels are shown in Figures 5.12 and 5.13 . Overall, the predictions between experiments and finite element simulations agreed well in terms level of through thickness failure (i.e nonpenetration, penetration, perforation) and shape of MCI de-bond surface.

Detailed investigation of contact force history for each impact velocity $\left(V_{i}\right)$ shows different level of influence caused by initial contact surface. The impact velocities 


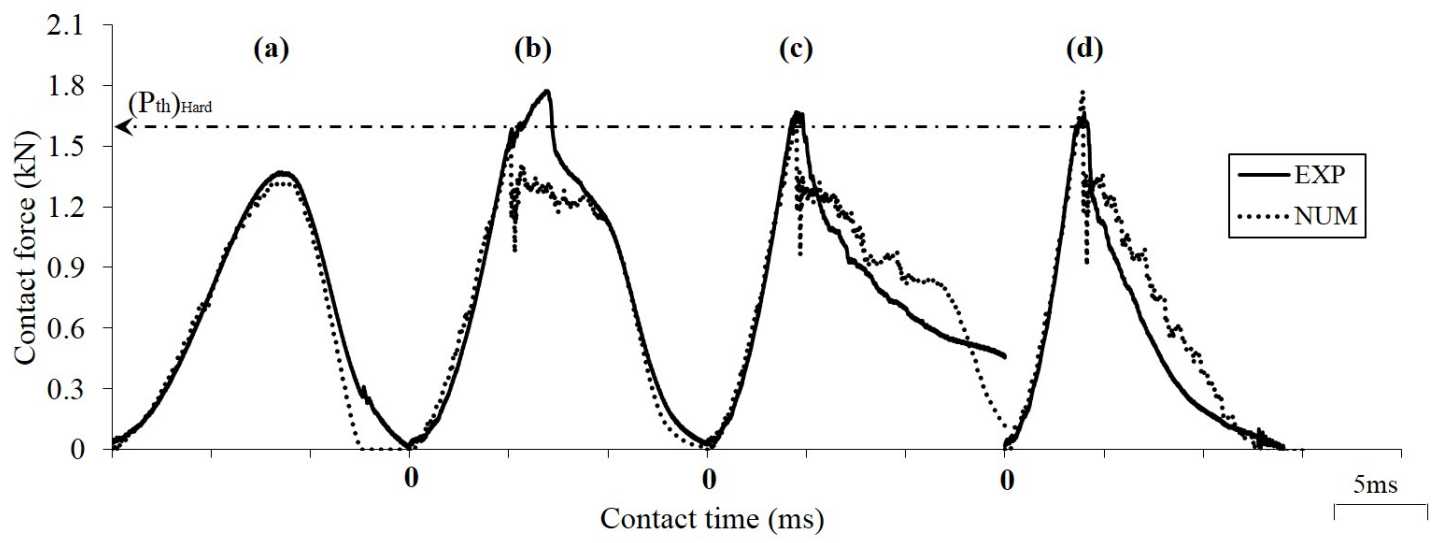

Figure 5.11: Experimental and numerical contact force histories of hard side impacted FML- $1 / 1$ panel (a) $V_{i}=1 \mathrm{~m} / \mathrm{s}$ (b) $V_{i}=1.5 \mathrm{~m} / \mathrm{s}$ (c) $V_{i}=1.75 \mathrm{~m} / \mathrm{s}$ and (d) $V_{i}=2 \mathrm{~m} / \mathrm{s}$
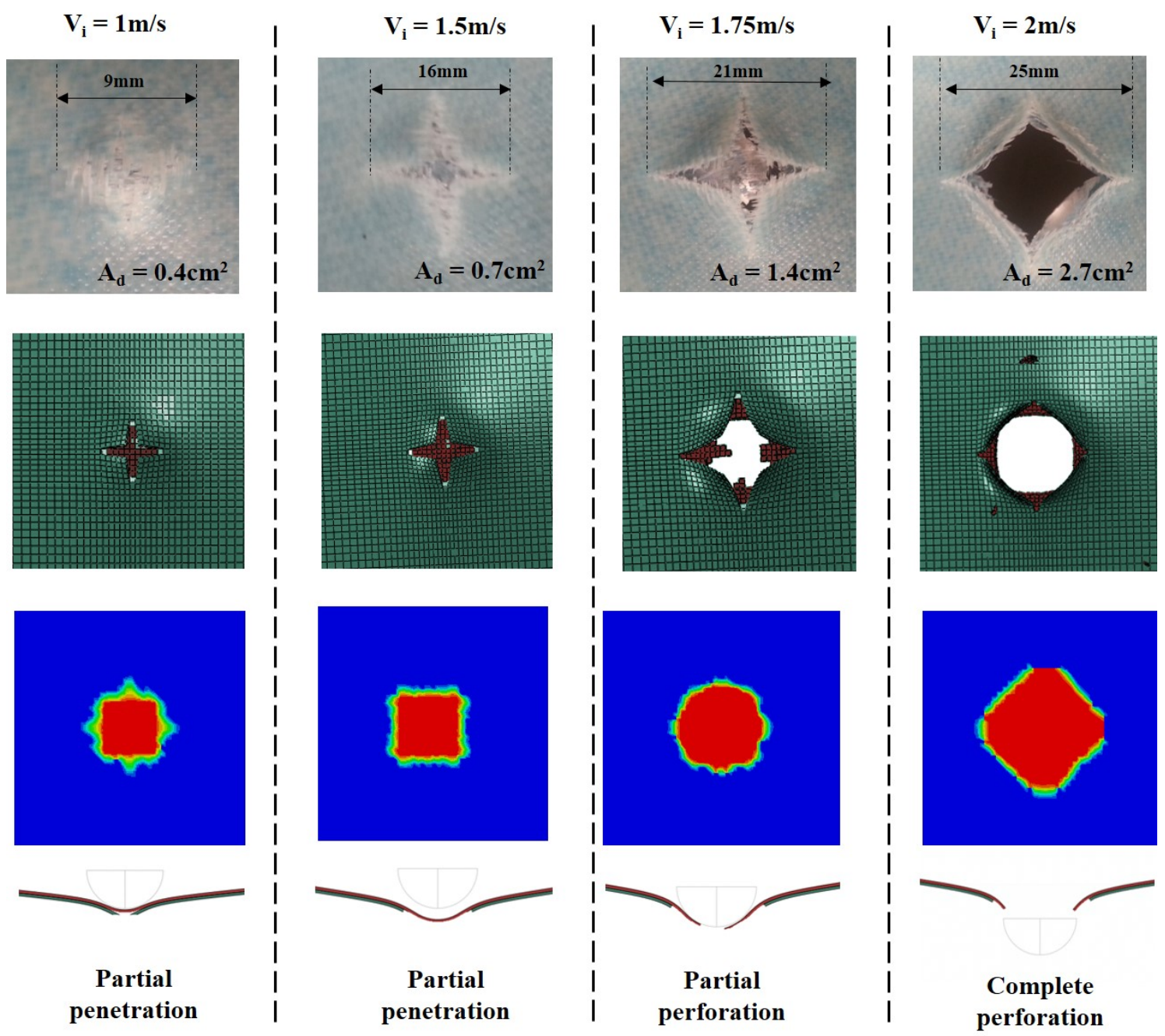

Figure 5.12: Experimental and numerical damage surface of soft side impacted FML-1/1 panel. First row- Experiment. Second row-Numerical. Third row-MCI damage(Numerical). Fourth row-Cross section(Numerical) 
are selected based on the energy level required to induce different stages of damage on soft side impact panels in QSI test.

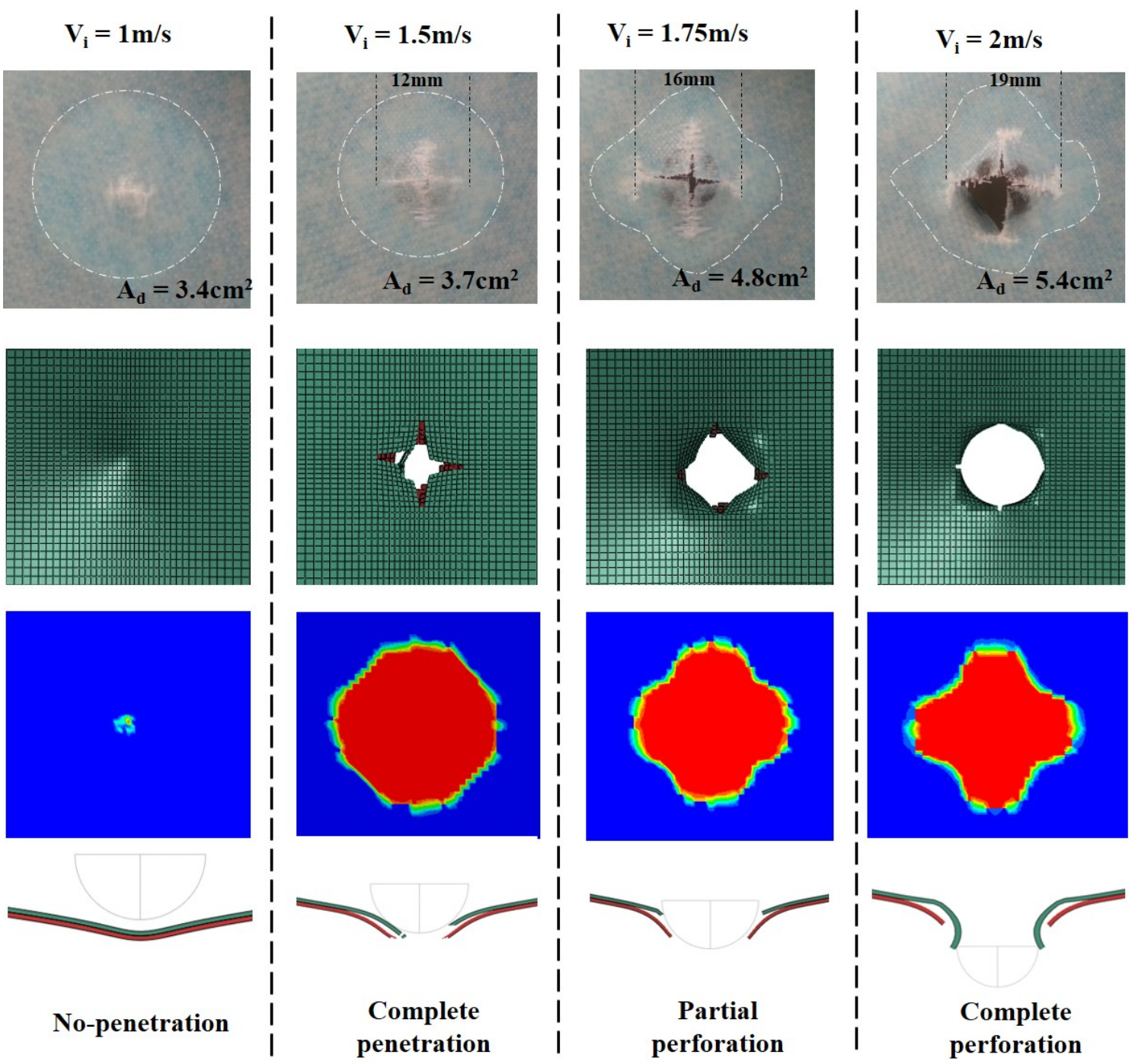

Figure 5.13: Experimental and numerical damage surface of hard side impacted FML-1/1 panel. First row- Experiment. Second row-Numerical. Third row-MCI damage(Numerical). Fourth row-Cross section(Numerical)

At higher impact velocities; $V_{i}=1.75 \mathrm{~m} / \mathrm{s}$ and $2 \mathrm{~m} / \mathrm{s}$ (energy level of stage III in QSI test), except the difference in threshold limit, the overall impact performance of soft and hard side impact panels is almost similar. Both the panels are perforated at this velocity range as can be seen in the damage surfaces with $V_{i}=1.75 \mathrm{~m} / \mathrm{s}$ and $2 \mathrm{~m} / \mathrm{s}$ in Figures 5.12 and 5.13 . On intermediate velocity range; $V_{i}=1.5 \mathrm{~m} / \mathrm{s}$ (energy level of stage II in QSI test), though peak contact force of soft side impact samples is lower than hard side impact sample, the latter yielded complete perforation 
while the former showed only partial penetration damage (see damage surfaces with $V_{i}=1.5 \mathrm{~m} / \mathrm{s}$ in Figures 5.12 and 5.13 .

Meanwhile in the low velocity range; $V_{i}=1 \mathrm{~m} / \mathrm{s}$ (energy level of stage II in QSI test), the impact response of hard side impact sample is better than soft side impact sample. The contact force curve is rather smooth and did not approached the threshold limit even though the impact energy is larger than the threshold energy level predicted in QSI test probably due to dynamic effects. Damage surfaces shows that none of the material layers are failed but there exists a small whitening region under the contact point seems to be matrix failure and notable MCI de-bond.

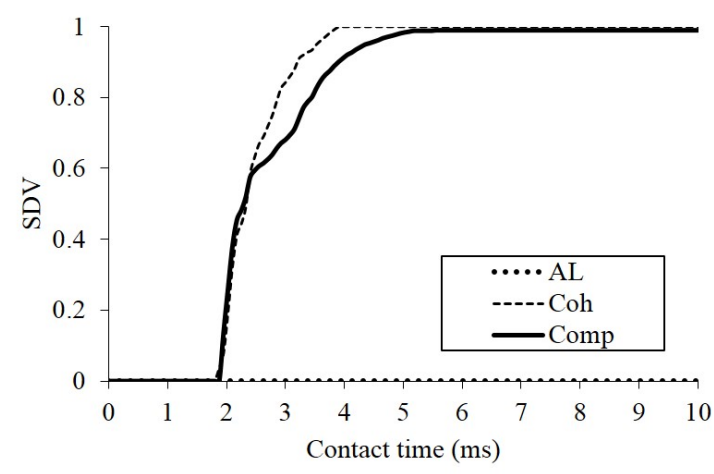

(a)

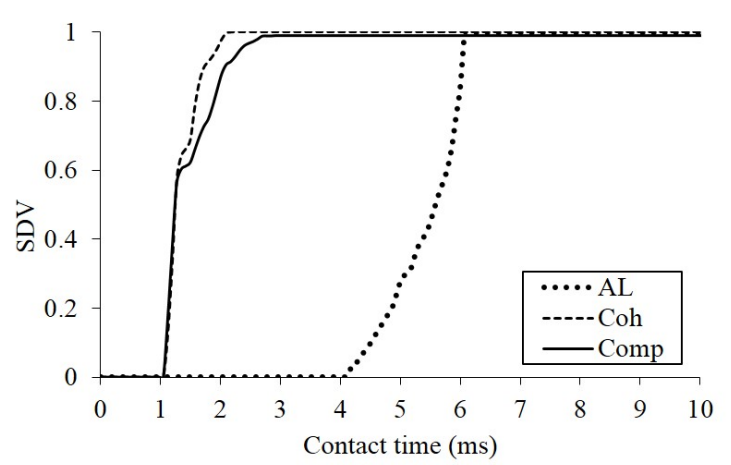

(c)

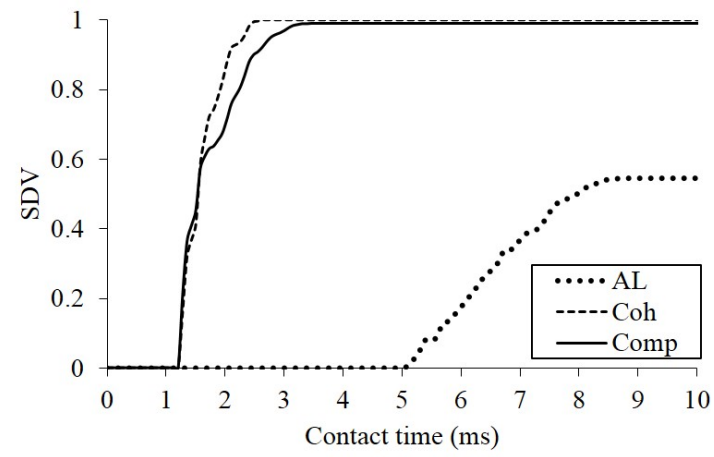

(b)

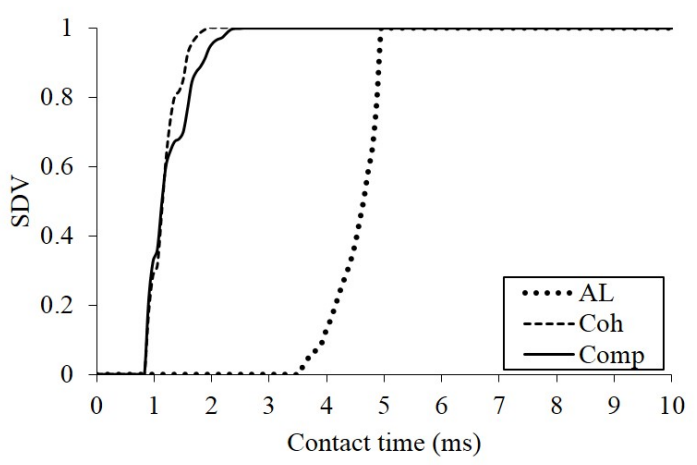

(d)

Figure 5.14: Damage evolution history of soft side impacted FML-1/1 panel (a) $V_{i}=1 \mathrm{~m} / \mathrm{s}(\mathrm{b}) V_{i}=1.5 \mathrm{~m} / \mathrm{s}(\mathrm{c}) V_{i}=1.75 \mathrm{~m} / \mathrm{s}$ and $(\mathrm{d}) V_{i}=2 \mathrm{~m} / \mathrm{s}$

Measurements of fibre crack length of impacted panels dictated that, increasing the impact velocity, the length of fibre crack in GFRP layers increases and even extend over longer length than the maximum contact diameter of $13.1 \mathrm{~mm}$ at higher impact velocities. Meanwhile, having backing layer and exposed in compression 
stress field, the fibre crack length of hard side impact samples are found to be consistently smaller than that of soft side impact sample.

From the MCI de-bond failure predictions of Figures 5.12 and 5.13 , the de-bond area $\left(A_{d}\right)$ induced in soft side impact sample is much smaller than that of hard side impact sample for all impact velocities. In soft side impact panels, the locus of de-bond was found to be highly controlled by fibre fracture of adjacent GFRP layer while in hard side contact sample, no such interrelationship was observed where debond boundary is found to be always greater than the fibre crack length of adjacent GFRP layer. Damage history predictions by state dependent variables (SDV) of material layers shown in Figures 5.14 and 5.15 reveals that, both MCI de-bond and composite layer damage occurs simultaneously for both impact surface cases followed by failure in the aluminum layer. It also shows that as the impact velocity increases, the time at which damage nucleated in each material layer occured earlier.

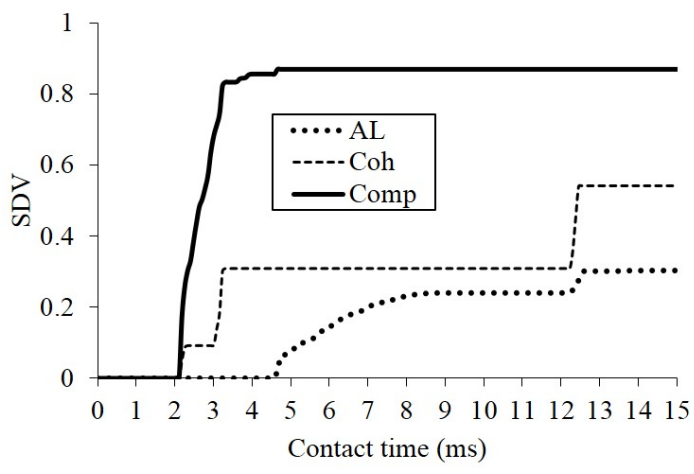

(a)

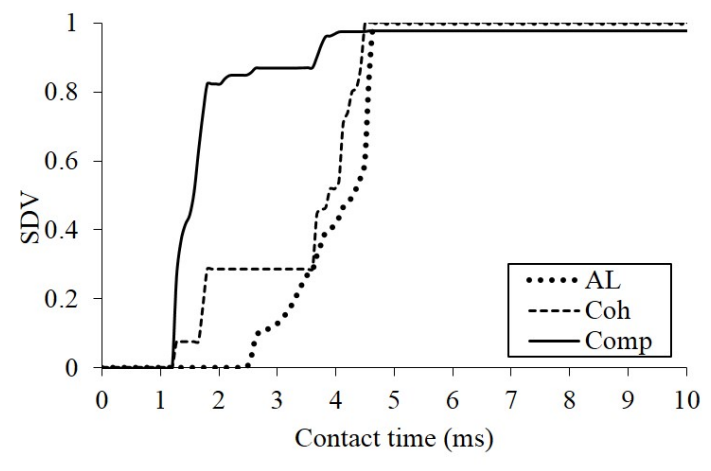

(c)

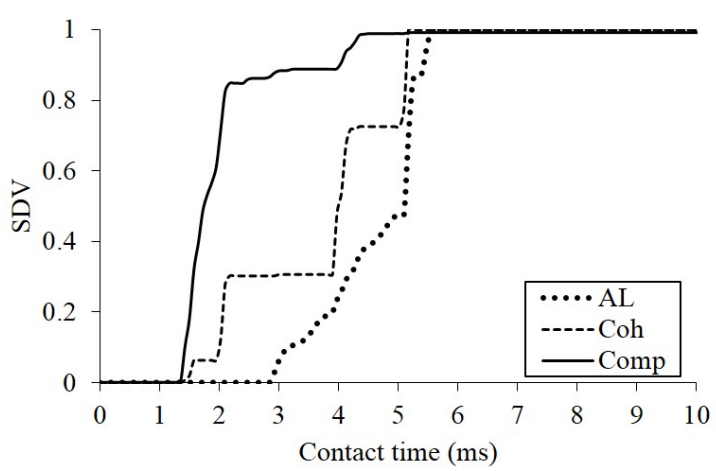

(b)

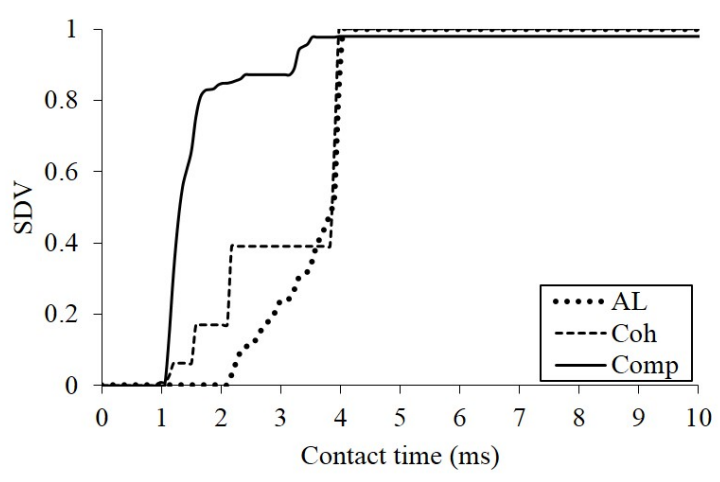

(d)

Figure 5.15: Damage evolution history of hard side impacted FML-1/1 panel (a) $V_{i}=1 \mathrm{~m} / \mathrm{s}(\mathrm{b}) V_{i}=1.5 \mathrm{~m} / \mathrm{s}(\mathrm{c}) V_{i}=1.75 \mathrm{~m} / \mathrm{s}$ and $(\mathrm{d}) V_{i}=2 \mathrm{~m} / \mathrm{s}$ 


\section{Energy Partition}

With reference to equation 5.4 , the impact energy in FML panel is dissipated by three phenomena; two irreversible and one partly reversible. The former two are energy absorbed by plastic deformation (PD) and by damage (DMD), and the latter refers energy absorbed by elastic recovery (EL). Figure 5.16 illustrates the variation of total absorbed energy (E_abs), and energy absorbed by different dissipation modes of soft and hard side impact samples at different velocity. As can be observed that with increase in impact velocity, the energy dissipated in the form of plastic dissipation by the aluminum layer and by forming new damage surfaces increases in both impact side cases. Also, the influence of the impact side on plastic deformation increases with increase in impact velocity. Accumulating more damage surface and plastic energy, the amount of energy absorbed in the form of recoverable strain energy (SE) is greatly reduced with increase in velocity and as a result the total absorbed energy by the FML panel is approximately equal to the incident impact energy.

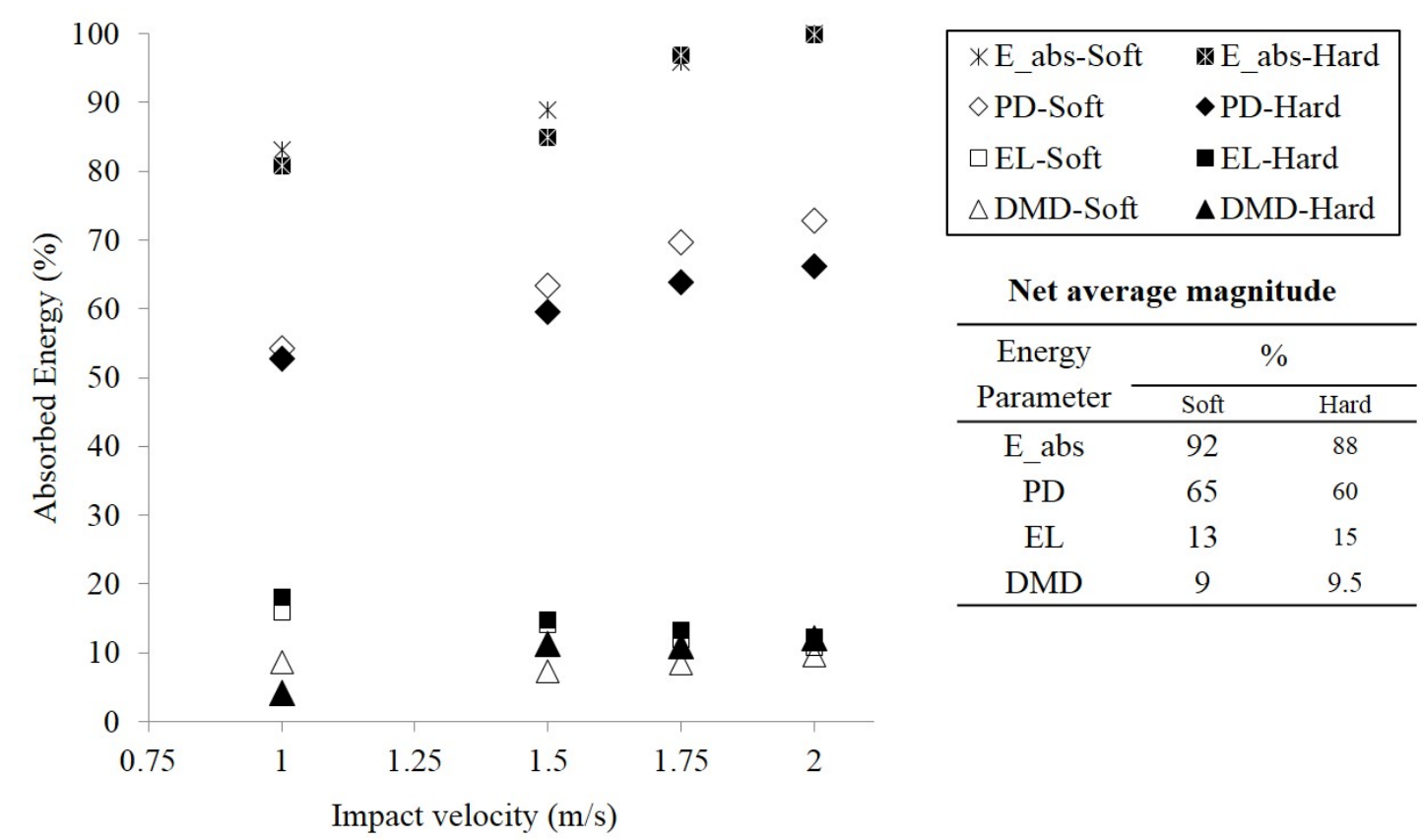

Figure 5.16: Energy partition of impacted FML-1/1 panel with different impact velocity (Table provides statistical average value)

The summary of net average energy magnitude listed in Figure 5.16 provides a general understanding of the role of material layers in FML-1/1 laminate. Over the 
range of impact velocity, this hybrid laminate is capable of absorbing nearly $\sim 90 \%$ of the incident impact energy ( $\sim 60 \%$ dissipated in the form plastic deformation, $\sim 20 \%$ by residual elastic energy and $\sim 10 \%$ to form new damage surfaces).

\subsubsection{Influence of MCI bonding}

For dissimilar material systems like FML, de-bonding along metal-composite interface (MCI) is caused by two factors:

(i) Mismatch in elastic stiffness between distinct heterogeneous material layers results in excessive shear along interfacial region. This results MCI de-bond nucleation via shear mode (Mode-II) as shown in Figure 5.17

(ii) Incompatible elastic recovery between permanently deformed plastic metal layer and elastically deformed composite layer during unloading (so called spring back) process results in MCI de-bond nucleation via open mode (ModeI) as shown in Figure 5.18 .

(a)

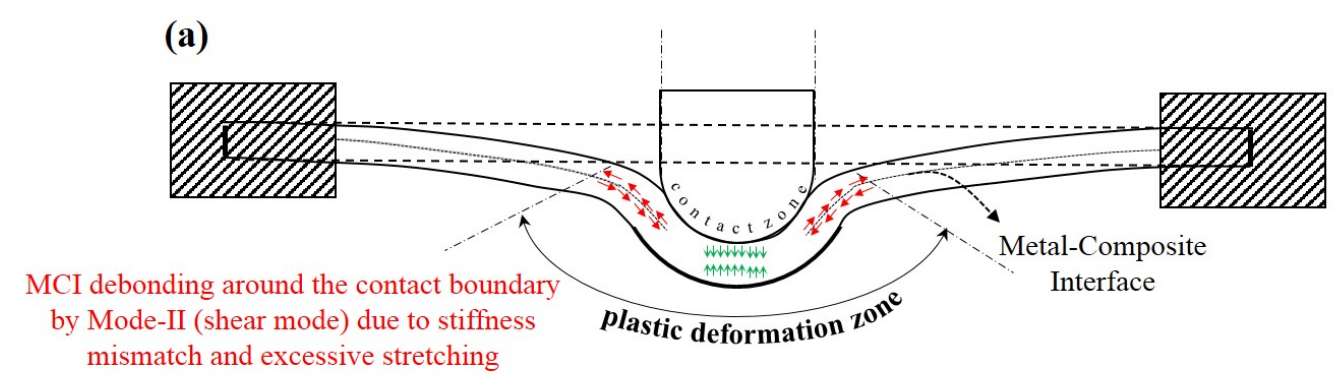

(b)

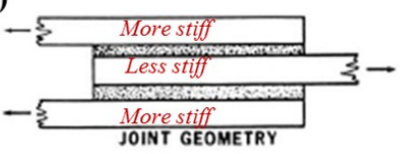

(c)

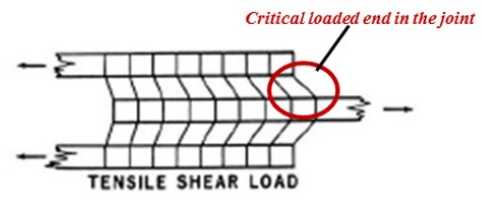

(d)

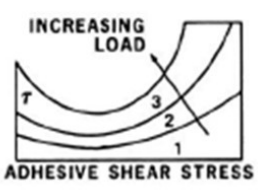

Figure 5.17: Phenomenon illustrating MCI de-bond nucleation by Mode-II fracture mode (a) Free body sketch (b) Elastically mismatched bonded layers (c) Influence of elastic mismatch (d) Curve showing shear stress concentration in the adhesive layer

Typical illustration explaining the above stated phenomena with FML-2/1 configuration is given in Figure 5.19. In general, de-bond initiation and nucleation are highly interacted with each other and the dominant fracture mode is difficult to 
estimate. However, the overall stiffness degradation arises from this failure mode is not significantly reflected in the resultant characteristic curves. A fraction of the incident energy utilized to cause such resin rich negligibly thin interfacial de-bonding in FML is generally small and accounts only around $1 \%$ as confirmed from Figure 5.8 and also pointed out elsewhere [138].

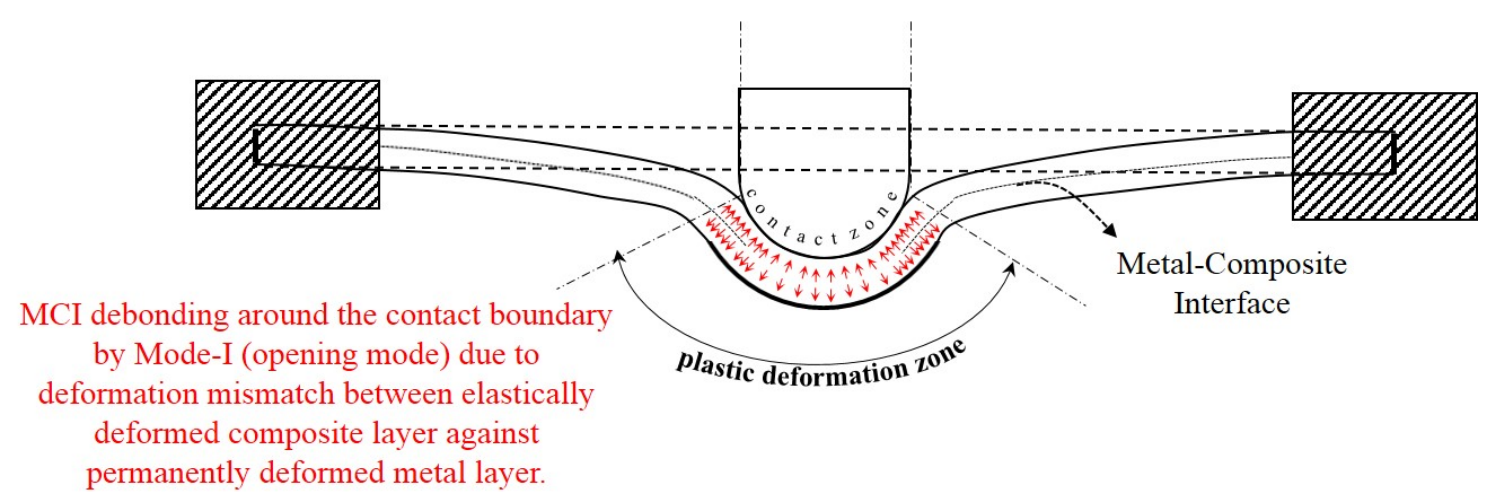

Figure 5.18: Phenomenon illustrating MCI de-bond nucleation by Mode-I fracture mode

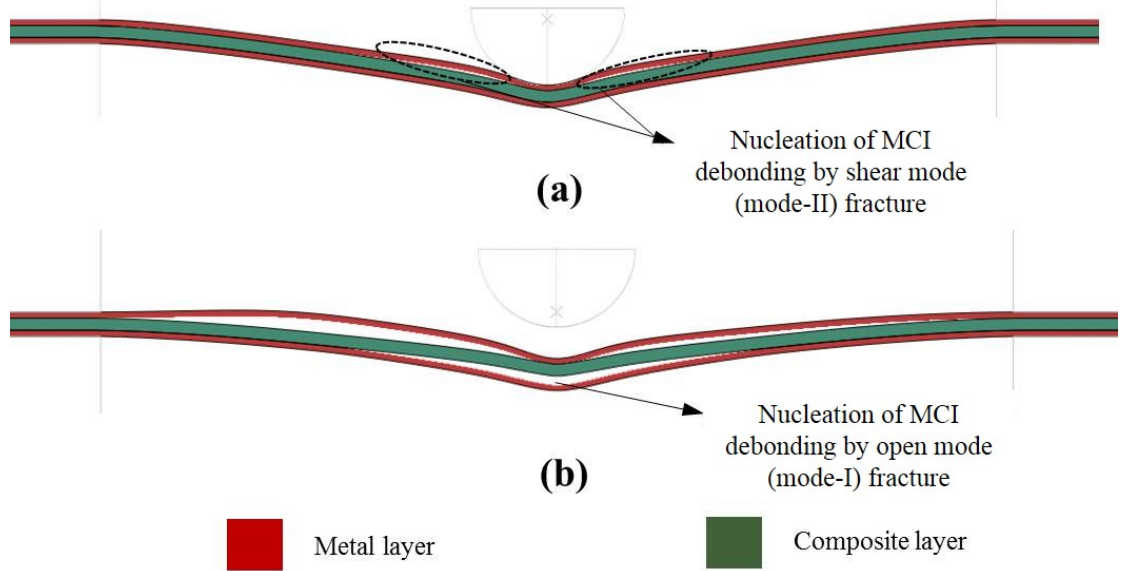

Figure 5.19: Numerically simulated cross sectional image showing two fracture modes of MCI de-bond damage in FML-2/1 panel (a) Mode-II (b) Mode-I

To better understand the role of MCI in FML system subjected to QSI and LVI loads, two categories of test samples are fabricated namely: (i) Self-bonded sample (ii) Pseudo-bonded samples. Information regarding stacking configuration and test conditions is listed in Table 5.6. 
Table 5.6: Configurations used to study influence of MCI bonding of FML-1/1 panel

\begin{tabular}{|c|c|c|c|c|c|c|}
\hline \multirow{2}{*}{ Sample } & \multirow{2}{*}{ Catergory } & \multirow{2}{*}{ Nomenclature } & \multirow{2}{*}{ Stacking sequence } & \multirow{2}{*}{ MVF } & \multicolumn{2}{|c|}{ Test condition } \\
\hline & & & & & QSI & LVI \\
\hline \multirow{4}{*}{ FML-1/1 } & \multirow{2}{*}{ Self } & Soft & $\mathrm{M} / \mathrm{C} / \mathrm{C}$ & 0.5 & \multirow{2}{*}{$1 \mathrm{~mm} / \mathrm{min}$} & $1 \mathrm{~m} / \mathrm{s}, 1.5 \mathrm{~m} / \mathrm{s}$ \\
\hline & & Hard & $\mathrm{C} / \mathrm{C} / \mathrm{M}$ & 0.5 & & $2 \mathrm{~m} / \mathrm{s}$ \\
\hline & \multirow{2}{*}{ Pseudo } & Soft & $\mathrm{M} / / \mathrm{C} / \mathrm{C} \ddagger$ & 0.5 & \multirow{2}{*}{$1 \mathrm{~mm} / \mathrm{min}$} & $1 \mathrm{~m} / \mathrm{s}, 1.5 \mathrm{~m} / \mathrm{s}$ \\
\hline & & Hard & $\mathrm{C} / \mathrm{C} / / \mathrm{M} \ddagger$ & 0.5 & & $2 \mathrm{~m} / \mathrm{s}$ \\
\hline
\end{tabular}

Note: $\mathrm{M}$ and $\mathrm{C}$ represents AL and GFRP respectively.

$\ddagger$ No adhesive bond between metal and composite layers.

\subsubsection{Response of soft side tested sample}

The higher initial slope in self-bonded sample as shown for QSI test in Figure 5.20(a) dictates that the flexural rigidity of the hybrid target is enhanced by improving the bonding between metal and GFRP layers. The threshold force at which stiffness degradation occurs is smaller for self-bonded sample $\left(P_{t h}\right)_{\text {self }}$ than pseudobonded sample $\left(P_{t h}\right)_{\text {pseudo }}$. Visual observation showed that the threshold condition in both samples are governed by the failure of the GFRP layer. The decrement in the threshold force of self-bonded sample $\left(P_{t h}\right)_{\text {self }}$ is probably due to good transfer of contact load to the bottom GFRP layer via perfectly bonded MCI layer. This can increase the tensile stress on the bottom layer and accelerated the failure of GFRP layer in self-bonded sample. However, after the threshold, both categories of sample showed similar loading slope, constant peak force $\left(P_{\max }\right)$, indenter displacement and absorbed energy $\left(E_{\max }\right)$ magnitude. This similar response is attributed to the fact that the post threshold response on these panels are solely contributed by the undamaged metal layer contribution. For a given indenter displacement, the final QSI damage surface shown in Figure 5.20(b) is similar for both cases except there exists a small difference in the fibre crack length on the GFRP layer.

The contact force history of self and pseudo-bonded FML-1/1 panel impacted on soft side at different impact velocity is shown in Figure 5.20(c). Impact velocities $\left(V_{i}\right)$ were selected with reference to amount of energy absorbed by each failure stages observed during QSI tests. It can be seen that, the threshold force remains constant for the range of impact velocity considered and the $\left(P_{\text {th }}\right)_{\text {pseudo }}$ is larger than the $\left(P_{t h}\right)_{\text {self }}$. For the impact energy close to that of threshold energy in QSI 


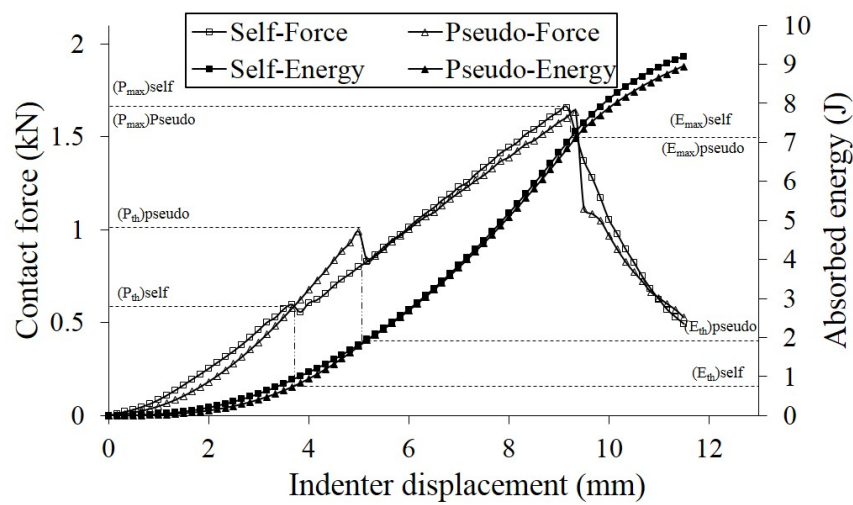

(a)

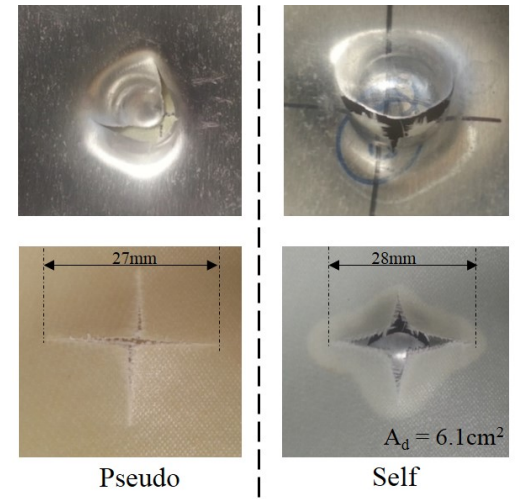

(b)

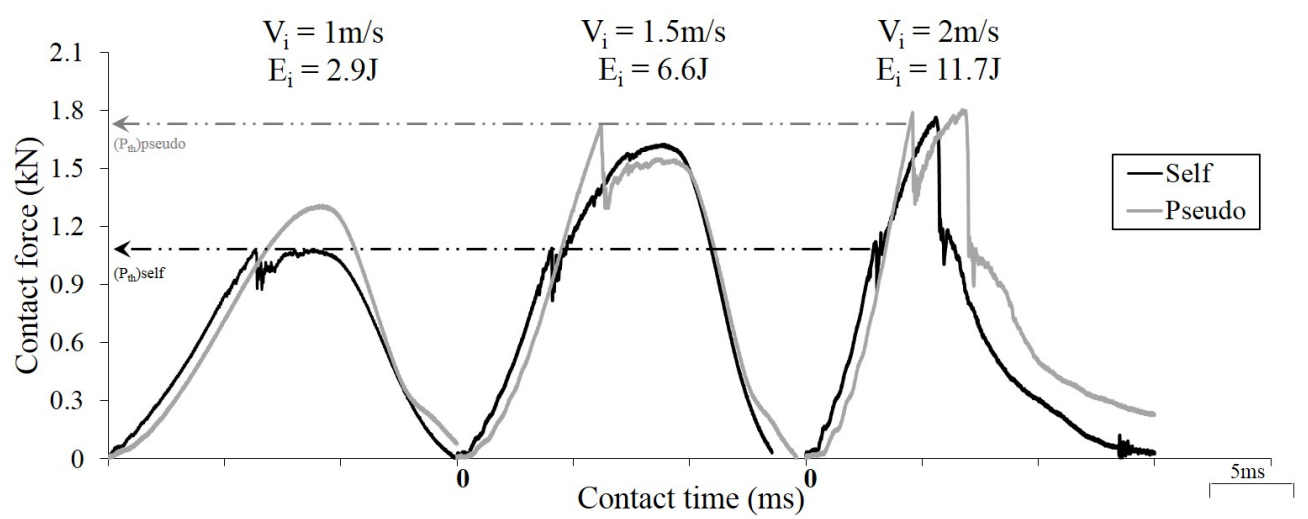

(c)

Figure 5.20: Indentation and impact response of self and pseudo-bonded soft side impact FML-1/1 panel (a) Indentation characteristic curve (b) Indented damage shape and (c) Impact characteristic curve

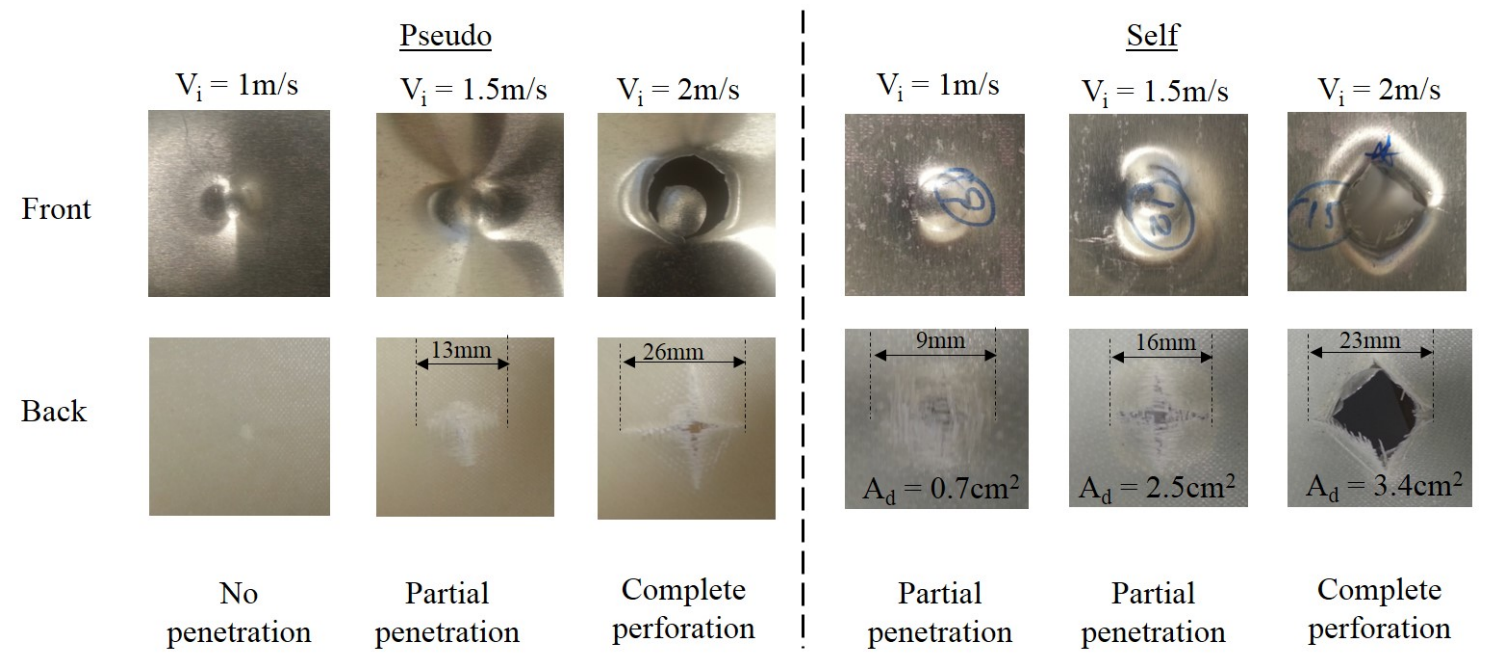

Figure 5.21: Experimental impact damage surface of self and pseudo-bonded soft side impact FML-1/1 panel 
test (i.e. $V_{i}=1 \mathrm{~m} / \mathrm{s}$ ), the force history of pseudo bonded samples shows smooth variation and no sign of threshold condition. Impact damage surfaces shown in Figure 5.21 confirmed that there is no sign of damage on GFRP layer of pseudo bonded sample but apprent fibre fracture in self-bonded sample. This confirms that the stress level in GFRP layer of pseudo-bonded sample is smaller than that in the corresponding self-bonded sample to initiate fibre fracture. At higher impact energy levels equivalent to post threshold energy level in QSI test (i.e. $V_{i}=1.5 \mathrm{~m} / \mathrm{s}$ and $2 \mathrm{~m} / \mathrm{s}$ ), except for the threshold condition, the response become almost similar (same $P_{\max }$ ) for both cases.

Comparing the damage surfaces of respective impact velocity as shown in Figure 5.21, it is evidenced that the absence of MCI de-bonding delays the failure initiation of the GFRP layer at lower velocity impact range. At higher (perforated) velocity range, though the GFRP layer of self-bonded sample underwent earlier failure, the fibre crack length is approximately constant. It seems that the influence of MCI become insignificant at higher velocity range due to high localized failure.

\subsubsection{Response of hard side tested sample}

Figure 5.22(a) shows the QSI response of hard side contact self and pseudo bonded samples. As can be seen, even though the threshold force of pseudo-bonded sample is slightly smaller than self-bonded sample, the indenter displacement at which the threshold condition arises and corresponding absorbed energy magnitude are approximately same. Refering to the slope of initial $\mathrm{P}-\delta$ curve, it is apparent that the small difference in force magnitude is because of the difference in flexural rigidity of the panel due to lack of bonding in pseudo FML panel.

Similar to the results of soft side contact panel, the threshold condition in the present case is also governed by failure of the GFRP layer. Furthermore, smaller barrier between the threshold limits with constant threshold energy level suggests that the influence of MCI bonding on damage threshold in hard side contact sample is not significant. However, it displays strong influence in post threshold condition. In addition with imposing larger flexural rigidity, firm bonding of GFRP layer 
substantially constrains the plastic flow tendency of metal layer in soft side contact sample and eventually penetrate with smaller peak force $\left(P_{\max }\right)_{\text {self }}$ at smaller indenter displacement $\left(\delta_{\max }\right)$. Damage surfaces shown in Figure 5.22 (b) displays similar morphology in terms of shape and it seems MCI bond arrest the fibre crack of GFRP layer by utilizing part of the load to develop interfacial de-bonding.

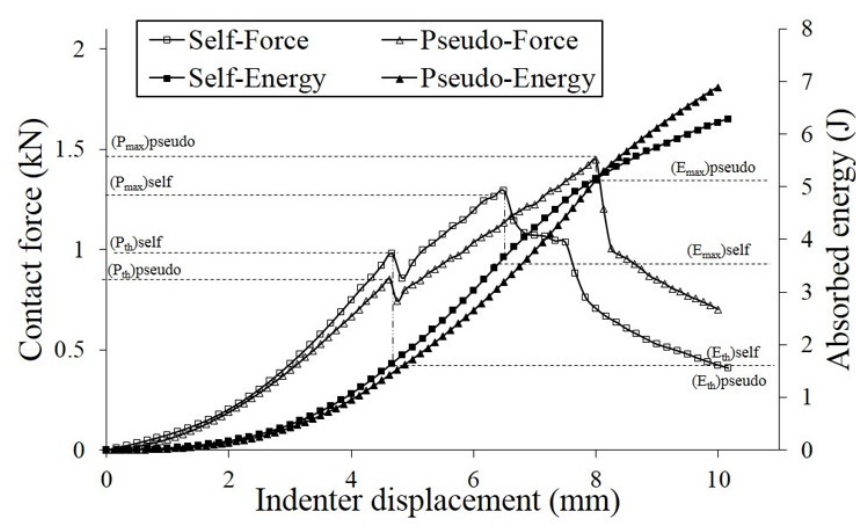

(a)
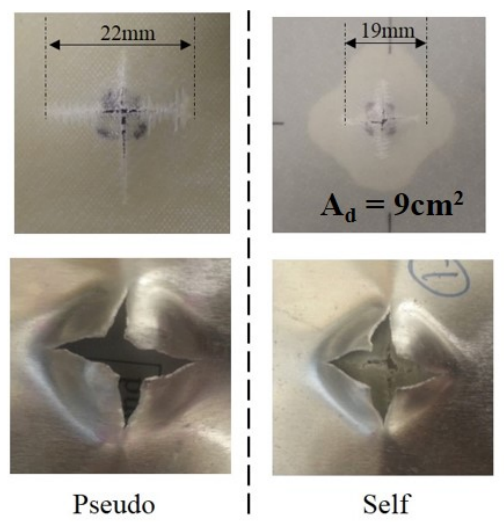

(b)

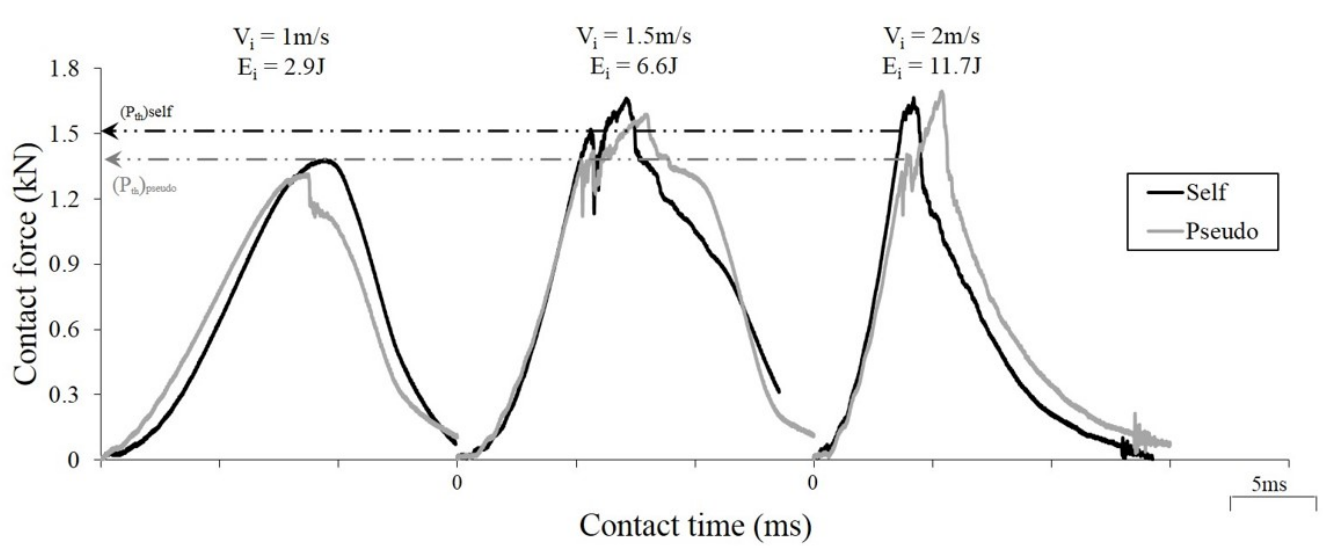

(c)

Figure 5.22: Indentation and impact response of self and pseudo-bonded hard side impact FML-1/1 panel (a) Indentation characteristic curve (b) Indented damage shape and (c) Impact characteristic curve

The impact response of hard side contact samples illustrated in Figure 5.22(c) shows similar sort of behaviour with smaller threshold force for pseudo bonded sample than for self-bonded sample. At lower impact velocity, self-bonded sample showed no sign of threshold with smooth contact force distribution compared to that of the pseudo-bonded sample. Meanwhile at higher velocity, the peak impact force is almost similar unlike the QSI response. However, the contact time at which 


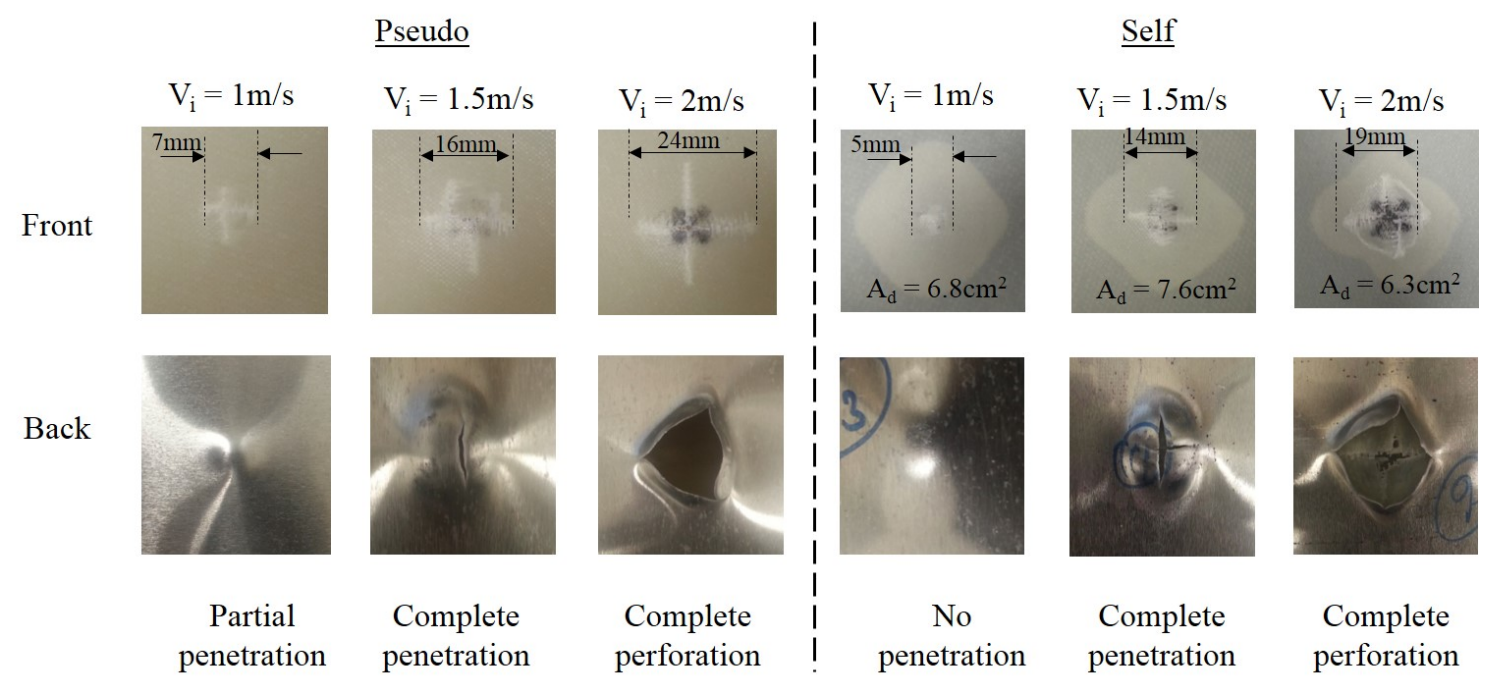

Figure 5.23: Experimental impact damage surface of self and pseudo-bonded hard side impact FML-1/1 panel

the self-bonded panels achieve the peak contact force is smaller than corresponding pseudo-bonded samples. This ascertains that perforation damage in the former samples are initiated earlier than that in the latter samples.

Figure 5.23 compares the damage surfaces of impacted panels of each impact velocity. As can be observed, except notable MCI de-bond surface and small whitening matrix crack surface under the contact region, no sign of fibre fracture visible on self-bonded sample but, an apparent fibre fracture is visible on the pseudo-bonded sample carries. At larger velocities, fracture surfaces and its intensities (penetration/perforation) are similar for both samples. Nevertheless, the presence of MCI bonding arrest the crack growth of fibres on bonded GFRP layer where fibre crack length of self-bonded samples are marginally smaller compared to that of pseudobonded sample. Efficient backing provided by metal layers through perfect MCI bonding might be the possible reason for the above damage scenario where the ductile backing allows the GFRP layer to undergo more bending deformation rather than to nucleate extended damage surface. 


\subsection{Indentation and impact behaviour of FML- $2 / 1$ panel}

This section attempts to extend the investigation of FML-1/1 configuration to conventional FML-2/1 configuration. Indentation and impact behaviour of FML-2/1 panel to different impact velocity and to different MCI surface are studied. Different kinds of failure surfaces observed from QSI and LVI tests were analyzed based on four distinct damage morphologies as shown in Figure 2.4 proposed originally by Starikov [20];

(i) Dent Damage (DD) - Damage on internal composite layer with visible dent on impacted surface.

(ii) First crack (FC) - DD + Visible crack on the back (non-impact side) metal sheet.

(iii) Through The Thickness (TTT) crack - FC + visible crack on the front metal sheet.

(iv) Complete perforation.

\subsubsection{Influence of impact velocity}

The test conditions used to investigate the effect of impact velocity is summarized in Table 5.7. Before performing impact tests, the behaviour of FML-2/1 panel to localized contact load was investigated using static indentation test.

Table 5.7: Configurations used to study influence of impact velocity of FML-2/1 panel

\begin{tabular}{lcccc|c}
\hline \multirow{2}{*}{ Sample } & \multirow{2}{*}{ Catergory } & \multirow{2}{*}{ Stacking sequence } & \multirow{2}{*}{ MVF } & \multicolumn{2}{c}{ Test condition } \\
\cline { 5 - 6 } & & & QSI & LVI \\
\hline FML-2/1 & \multirow{2}{*}{ Bond } & M/A/C $/ \mathrm{C} / \mathrm{C} / \mathrm{C} / \mathrm{A} / \mathrm{M}$ & 0.5 & $1 \mathrm{~mm} / \mathrm{min}$ & $\begin{array}{l}1.5 \mathrm{~m} / \mathrm{s}, 1.75 \mathrm{~m} / \mathrm{s} \\
3 \mathrm{~m} / \mathrm{s}, 3.25 \mathrm{~m} / \mathrm{s}\end{array}$ \\
\hline
\end{tabular}

Note: M, A and C represents AL, RE and GFRP respectively. 


\subsubsection{Quasi-static indentation response}

Figure 5.24(a) illustrates the contact force and accumulated absorbed energy versus indenter displacement of statically indented FML-2/1 panel. After some extent, contact force experiences a severe non-linear response that signifies initiation of plastic deformation in aluminium layers. The first damage in the panel is governed by failure of internal composite layer (DD). However, their influence is not obvious in the force -displacement curves because the loss in stiffness originated due to composite layer failure is compensated by surrounding undamaged ductile aluminium layers. At an instant when the back aluminum layer fails (FC), load curve shows significant load drop but the FML panel manages to resists the contact load via the front aluminum layer. Then the front layer starts to crack (TTT) which reflects another significant drop in force-displacement curve. After that, indenter starts to perforate the panel and the contact load starts to decrease monotonically. Energy distribution curve shows that the energy required to nucleate composite damage (DD) in FML-2/1 is very small Compared to energy required to initiate FC and TTT damage.

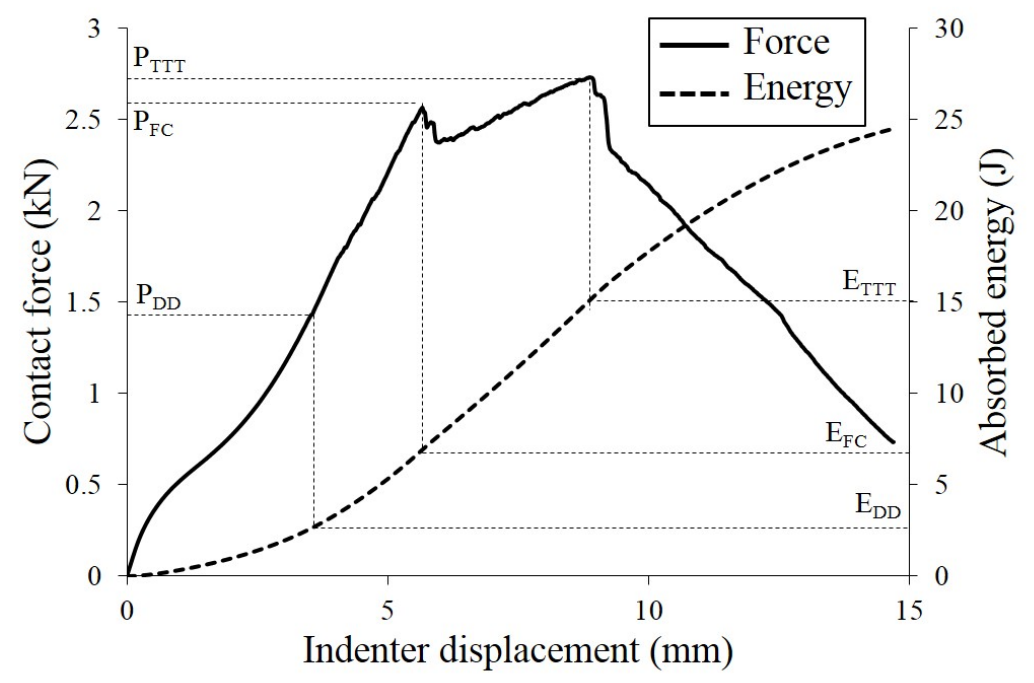

(a)
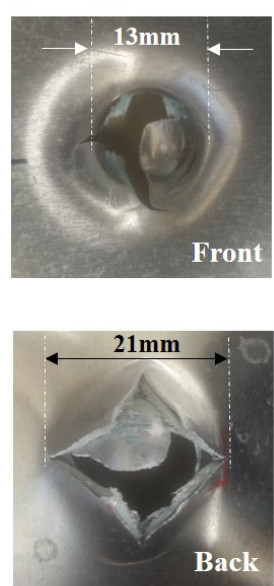

(b)

Figure 5.24: Indentation response of FML-2/1 panel. (a) characteristic curve and (b) indented damage shape 


\subsubsection{Low velocity impact response}

\section{Observed experimental response}

Based on the energy level to induce a particular damage shape in QSI test, respective impact velocities for impact test were selected. Figure 5.25 shows the contact force-time history for all impact velocities. As expected, slope of the loading curve increases with increase in impact velocity. It is interesting to note that the force magnitudes at which different damage morphology originates in FML-2/1 panel is constant regardless of the impact velocity.

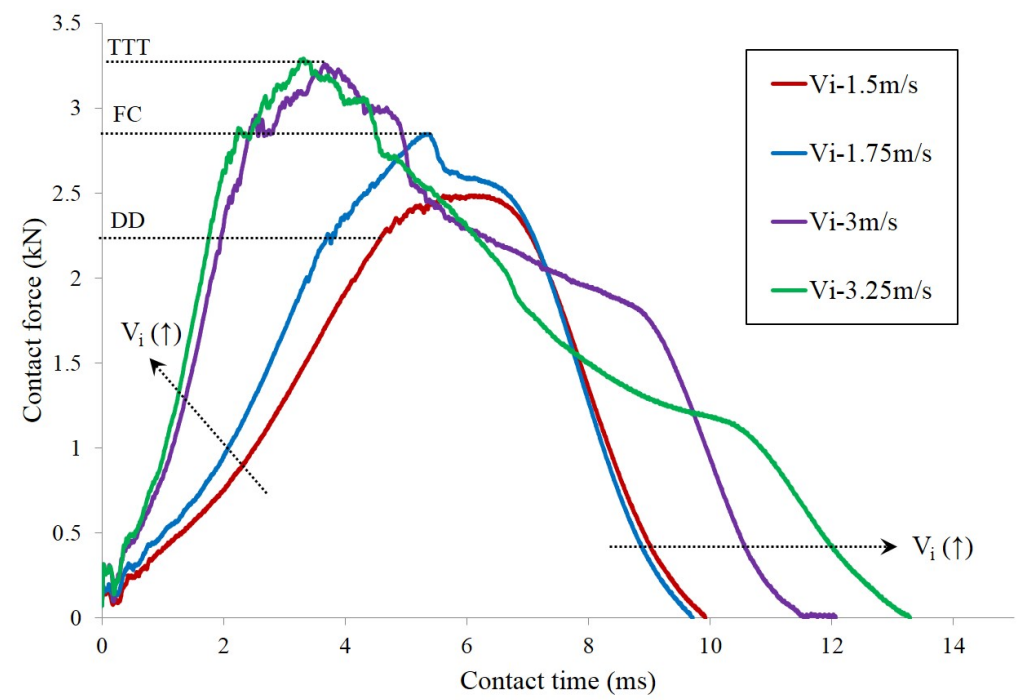

Figure 5.25: Impact response of FML-2/1 panel

After the nucleation of TTT damage, the impact response of the panel has a longer contact time with increase in impact velocity. This is because the flexural rigidity of panel has been extensively deteriorated at the instant when all the stacked layers of the panel are fractured and the intensity of damage is proportional to impact velocity. Induced impact damage surfaces of FML panel to each impact velocity is given in Figure 5.26. It can be seen that, both the indentation (see Figure $5.24 \mathrm{~b}$ ) and impact induced TTT damage surfaces are similar. The back aluminum layer produces a petaling failure, whose crack fronts are closely associated with fibre crack of adjacent composite layer while the front aluminium layer fails via dishing with circular crack pattern around the contact boundary induced due to severe radial 
tensile stress 139. The measurement of contact impressions shows that the contact radius of impactor increases with increase in impact velocity and reaches a maximum value of impactor diameter at perforation velocity level. However, the crack length of the back aluminum layer is far greater than the contact radius of impactor for all velocity cases.
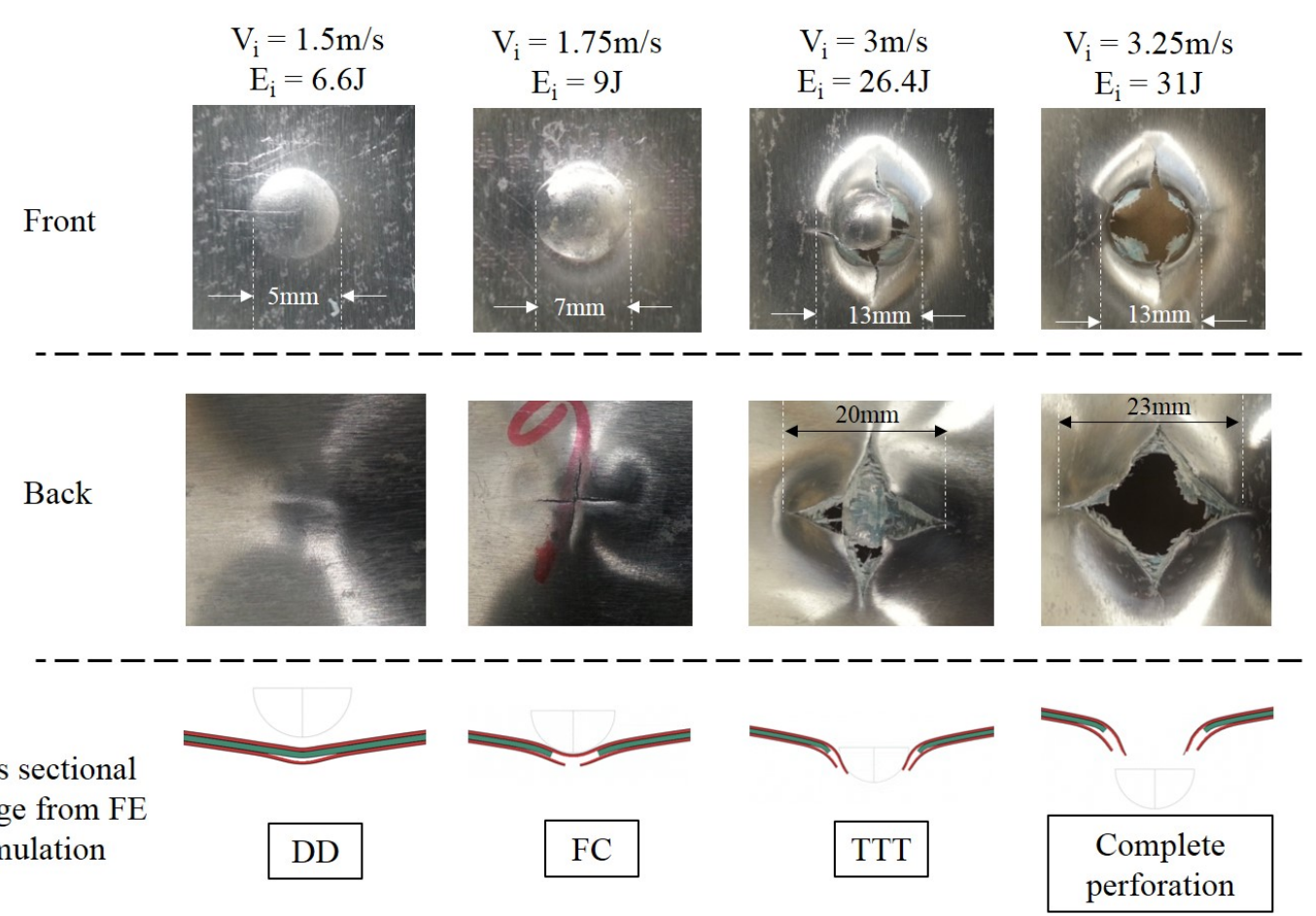

Figure 5.26: Impact damage surface of FML-2/1 panel. First row- Front (impact) side. Second row-Back (non-impact) side. Third row- Simulated cross section

\section{Predicted numerical response}

Figure 5.27 show the comparison between the experimental and the numerical variation of the contact force history of the FML-2/1 laminate for different impact velocity. As can be observed that the loading curve, different failure stages and maximum load are well predicted while the unloading curve especially for the panel with TTT damage is highly under predicted. As with the experimental observations, the numerically predicted threshold force magnitude to cause different failure stages (DD, FC and TTT) are same for all impact velocity.

The deviation in unloading contact force prediction is suspected due to ero- 


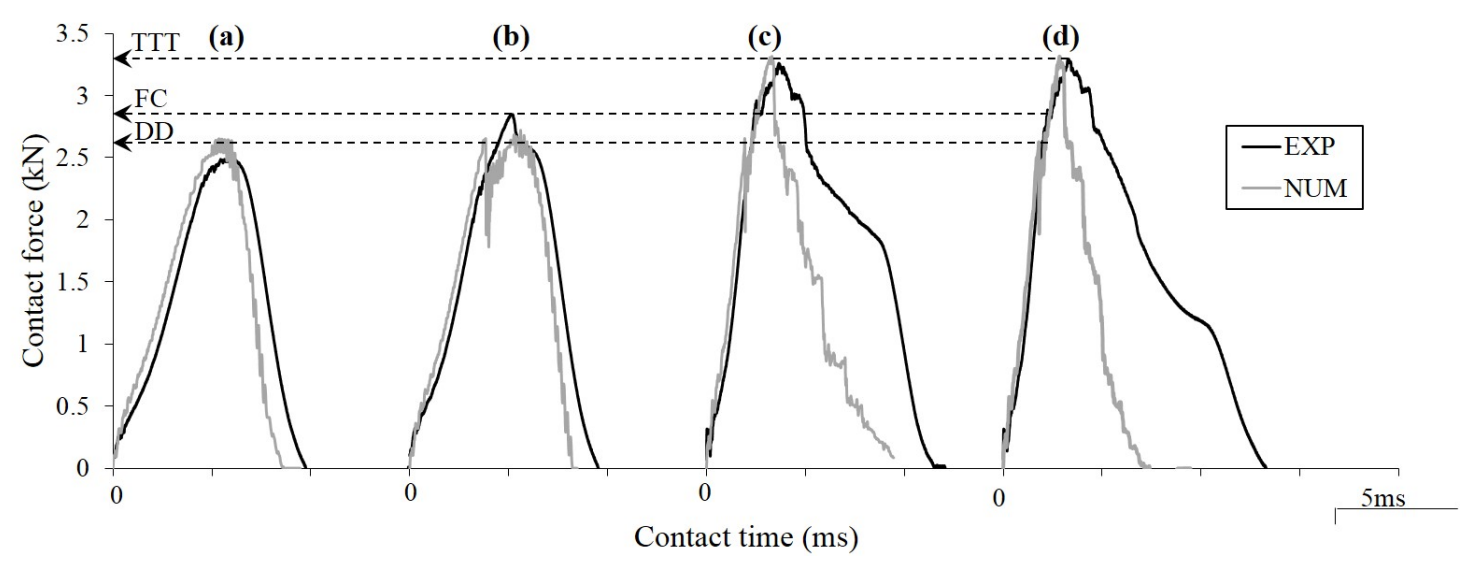

Figure 5.27: Experimental and numerical contact force histories of FML-2/1 panel. (a) $V_{i}=1.5 \mathrm{~m} / \mathrm{s}$ (b) $V_{i}=1.75 \mathrm{~m} / \mathrm{s} \mathrm{(c)} V_{i}=3 \mathrm{~m} / \mathrm{s}$ and (d) $V_{i}=3.25 \mathrm{~m} / \mathrm{s}$

sion phenomenon implemented in the numerical model by which the failed elements are completely removed from the element cluster soon after it satisfies the damage evolution condition. However, the stated scenario is not actually the case in real experiments where the failed fragments remain with in the parent material and contribute some stiffness to the failed structure. Nevertheless, the final failure stage was well predicted for all impact velocities as compared in Figure 5.26 .

With respect to the initial contact surface, MCI interfacial regions between metal and composite layers of FML has been further classified in to two types: MetalComposite (M-C) and Composite-Metal (C-M) region as shown in Figure 5.28(a). In accordance with that nomenclature, MCI region of FML-2/1 panel are entitled as M-C (between front metal and composite layer) and C-M (between composite and back metal layer) interface. Figure 5.28(b) shows the contour images of de-bond extension boundaries of FML-2/1 panel for all impact velocities. As shown, the de-bond area of $\mathrm{C}-\mathrm{M}$ interface is always larger than $\mathrm{M}-\mathrm{C}$ interface except for very low impact velocity level of $V_{i}=1.5 \mathrm{~m} / \mathrm{s}$. This scenario is reflected even in FML-1/1 panel (shown in Figures 5.12 and 5.13) where de-bond area of M-C interface of soft side impact sample is smaller than C-M interface of hard side impact sample for the given impact velocity and MVF.

The damage evolution history tracked through respective state dependent variable (SDV) for each layer is illustrated in Figure 5.29. It can be seen that, with increase in impact velocity, the order of damage nucleation in FML-2/1 panel is 


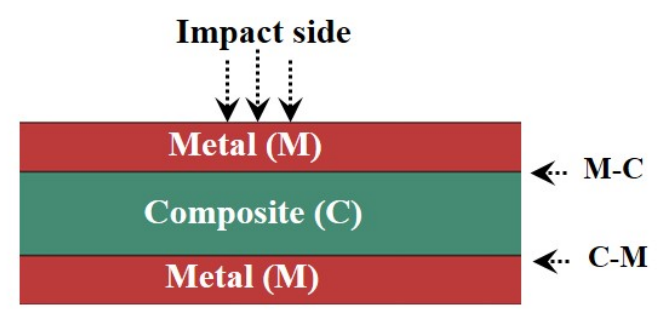

(a)

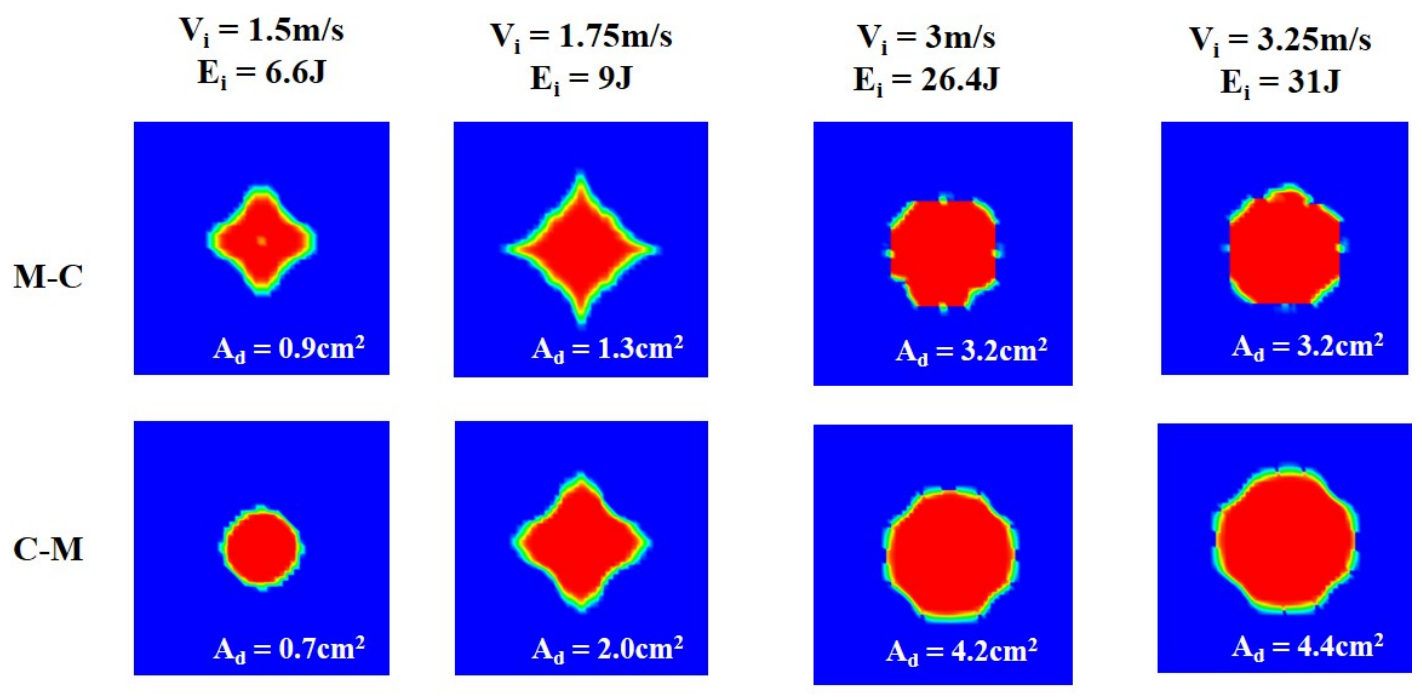

(b)

Figure 5.28: Numerically predicted MCI de-bond damage surface of FML-2/1 panel. (a) Nomenclature of MCI interface (b) Predicted MCI de-bond contours 
the same. The sequence of fracture induced due to impact load takes the following order; top cohesive interface layer (M-C interface between front aluminium and composite layer), middle composite layer, bottom cohesive interface layer ( $\mathrm{C}-\mathrm{M}$ interface between composite and back aluminum layer), back aluminium and finally front aluminium layer. The above sequence of interfacial damage in FML-2/1 panel is somewhat consistent with damage scenario of FML-1/1 panel. It can be seen from Figure 5.8 that, the interfacial regions of soft side impact sample having M-C interface de-bond earlier than C-M interface of hard side impact sample.

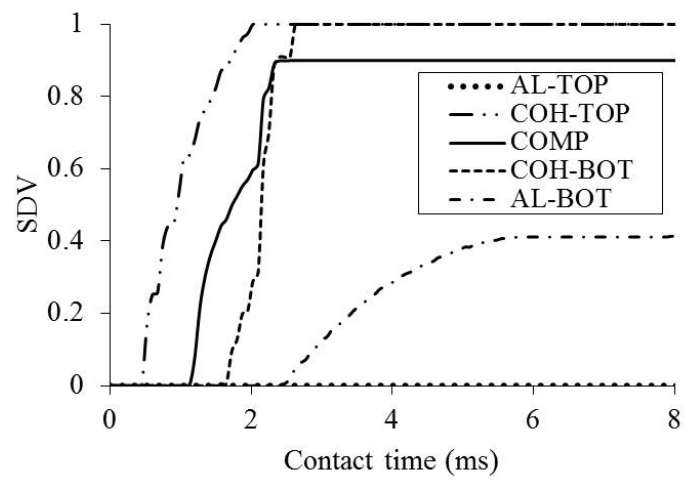

(a)

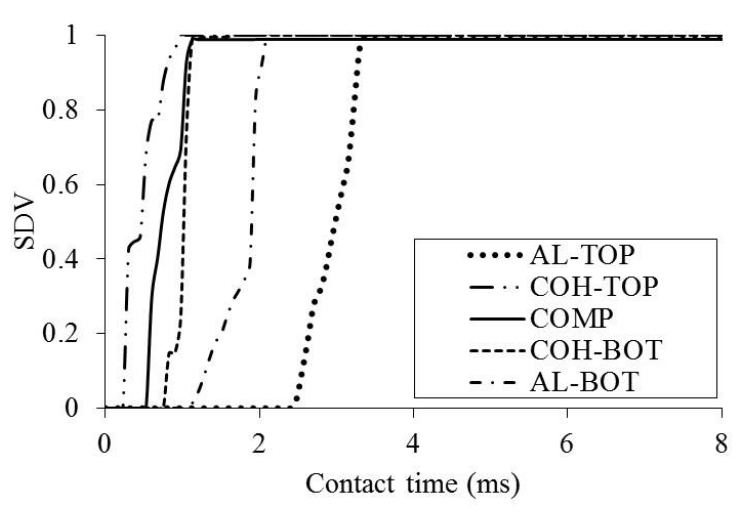

(c)

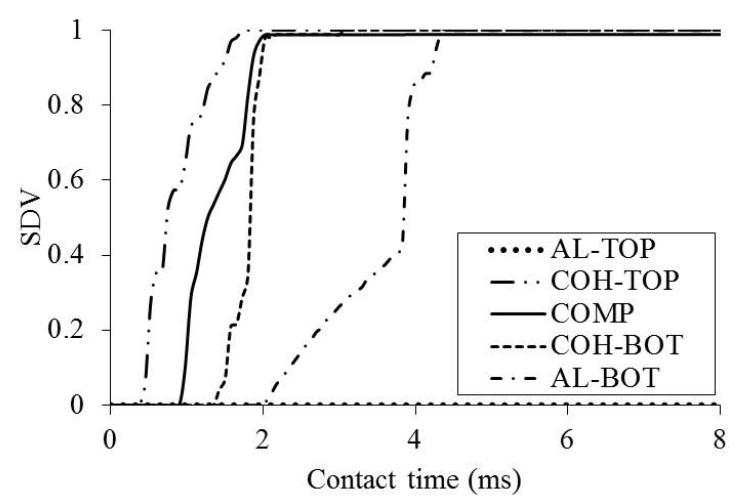

(b)

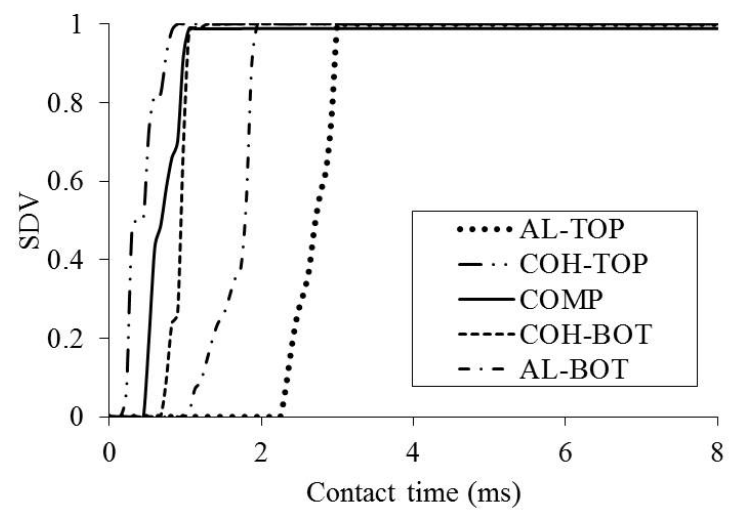

(d)

Figure 5.29: Damage evolution history of impacted FML-2/1 panel. (a) $V_{i}=1.5 \mathrm{~m} / \mathrm{s}$ (b) $V_{i}=1.75 \mathrm{~m} / \mathrm{s}$ (c) $V_{i}=3 \mathrm{~m} / \mathrm{s}$ and (d) $V_{i}=3.25 \mathrm{~m} / \mathrm{s}$

Finally, the quantity of mode-wise energy absorbed during the impact response for all tested velocities is plotted in Figure 5.30. With reference to equation (5.4), FML-2/1 absorbs nearly $90 \%$ of incident energy of which $65 \%$ is dissipated via plastic deformation of the aluminum layer, $13 \%$ by elastic energy in both aluminum and composite layer and $10 \%$ by forming new damage surface on whole FML laminate. 


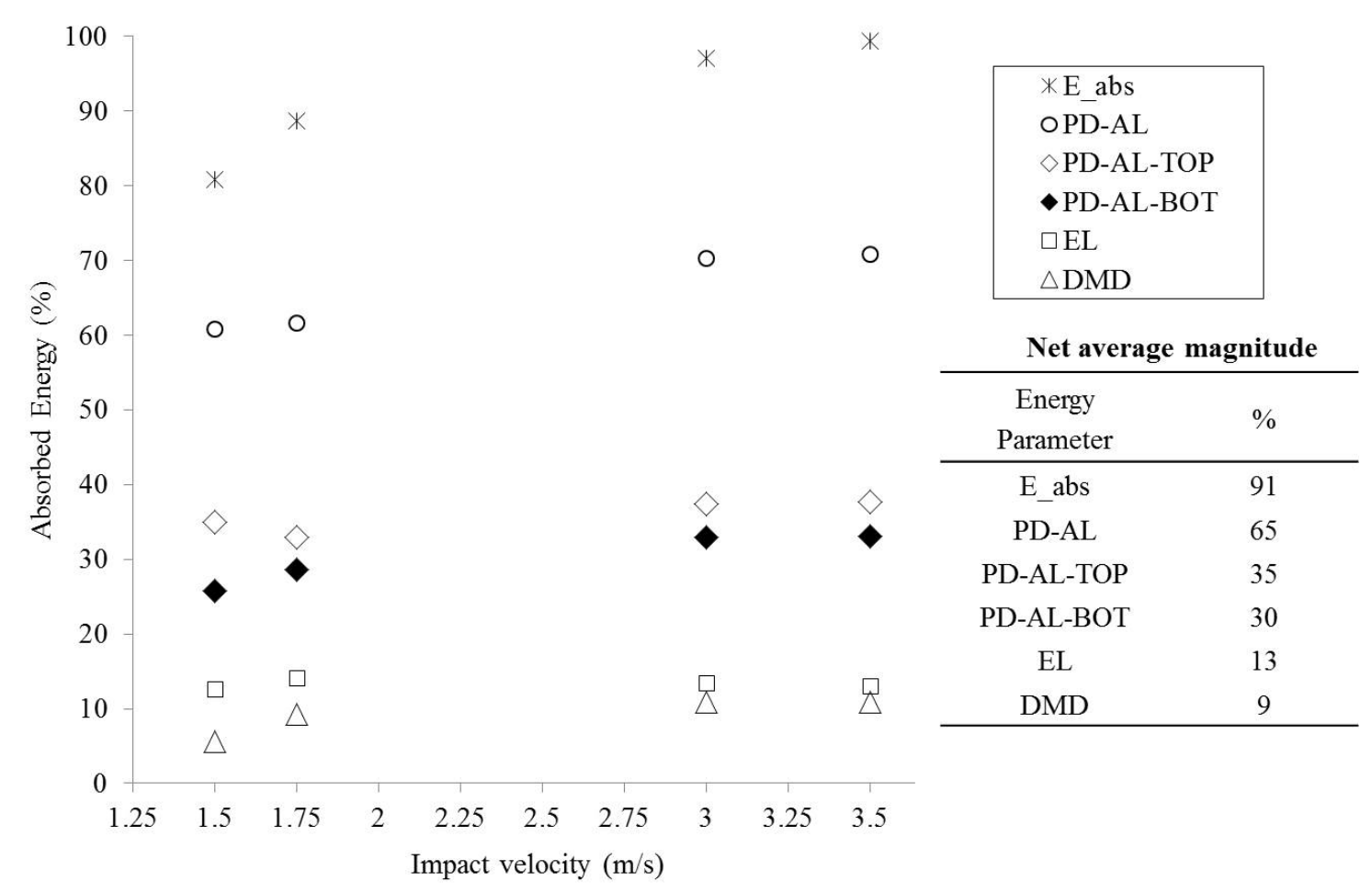

Figure 5.30: Energy partition of impacted FML-2/1 panel with different impact velocities (Table provides statistical average value)

It should be noted, nearly 2-3\% of the energy is lost through hourglass control in numerical simulation of all impact cases. The layer wise assessment of plastic energy shows that the, energy absorbed by the front aluminum (AL-TOP) layer is slightly larger than the back aluminum layer (AL-BOT) which is probably due to additional plastic dent deformation in the front layer. Also with increase in velocity, the energy dissipated by plastic deformation and by damage formation increases while the elastically absorbed energy remains constant.

\subsubsection{Influence of MCI bonding}

Self and pseudo-bonded panels were used to investigate the influence of MCI bond in FML-2/1 configuration. Details of the stacking sequence and test conditions used in the present study is summarized in Table 5.8 .

\subsubsection{Quasi-static indentation response}

Comparison of load and energy with indenter displacement for self and pseudobonded FML-2/1 panel is given in Figure 5.31. It can be seen that, the absence of 
Table 5.8: Configurations used to study influence of MCI bonding of FML-2/1 panel

\begin{tabular}{lcccc|c}
\hline \multirow{2}{*}{ Sample } & \multirow{2}{*}{ Catergory } & \multirow{2}{*}{ Stacking sequence } & \multirow{2}{*}{ MVF } & \multicolumn{2}{c}{ Test condition } \\
\cline { 6 - 6 } & & & QSI & LVI \\
\hline \multirow{2}{*}{ FML-2/1 } & Self & M/C $/ \mathrm{C} / \mathrm{C} / \mathrm{C} / \mathrm{M}$ & 0.5 & \multirow{2}{*}{$1 \mathrm{~mm} / \mathrm{min}$} & $1.75 \mathrm{~m} / \mathrm{s}, 2 \mathrm{~m} / \mathrm{s}$ \\
& Pseudo & M/ $/ \mathrm{C} / \mathrm{C} / \mathrm{C} / \mathrm{C} / \mathrm{M} \ddagger$ & 0.5 & & $2.5 \mathrm{~m} / \mathrm{s}, 3 \mathrm{~m} / \mathrm{s}$ \\
\hline
\end{tabular}

Note: $\mathrm{M}$ and $\mathrm{C}$ represents AL and GFRP respectively.

$¥$ No adhesive bond between metal and composite layers.

MCI bonding immensely deteriorated the contact stiffness of pseudo-bonded sample. Indeed, on comparing Figure 3.6, initial contact response of pseudo-bonded sample was found rather similar to that indentation response of pure aluminium sample. It meant layers of pseudo-bonded panel were independently deformed due to lack of bonding and thus the initial behaviour is accompanied only by initial contact surface.

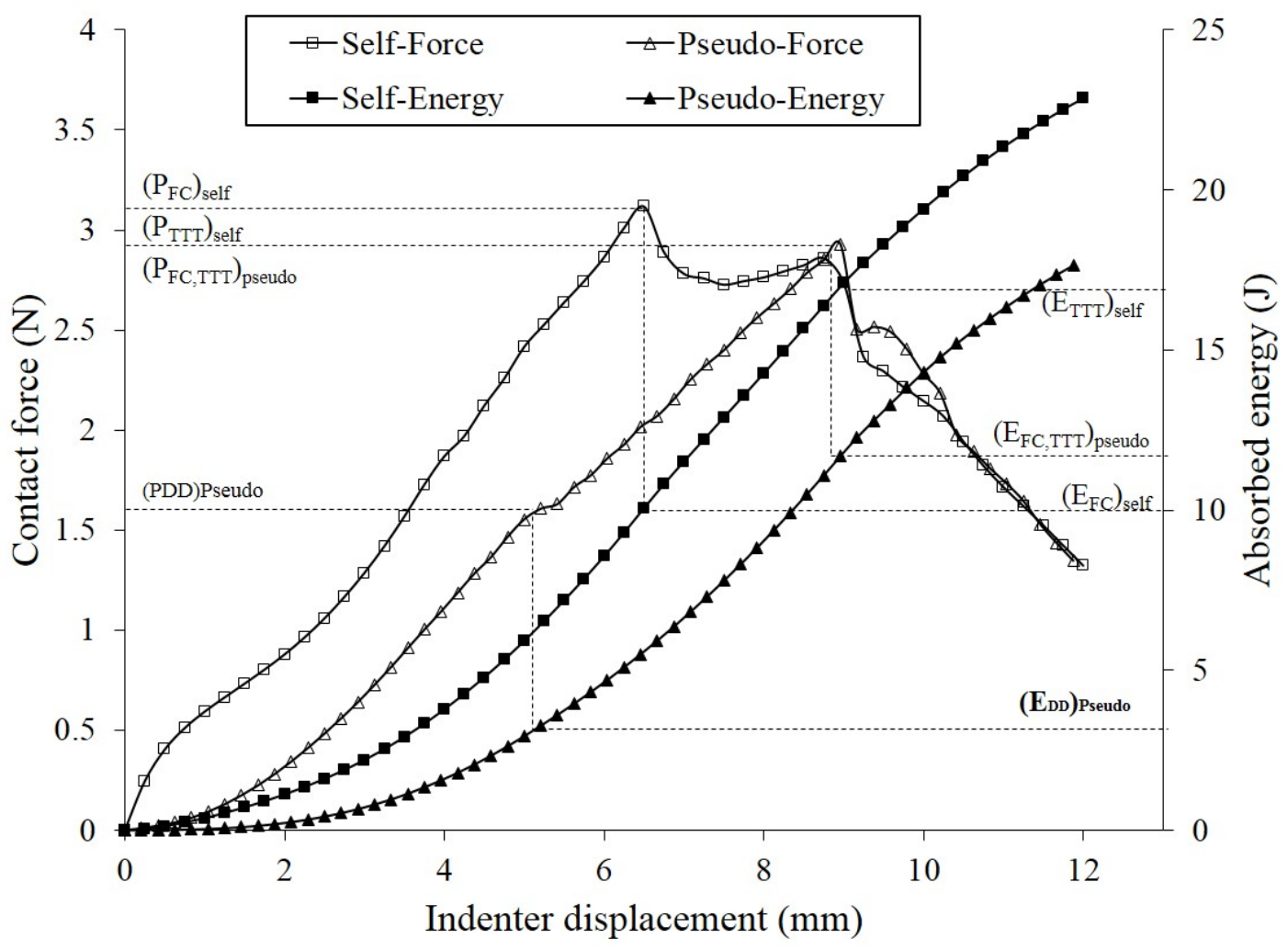

Figure 5.31: Indentation response of self and pseudo-bonded FML-2/1 panel

Identification of dent damage (DD) associated with failure of the composite layer is apparent in the force curve of pseudo-bonded sample showing change in stiffness but no sign of stiffness degradation in self-boned sample. This can be due to effective redistribution of the contact load to adjacent undamaged aluminium 
layers via bonded MCI interface. However, such load distribution phenomenon does not exist in pseudo-bonded due to lack of interface bonding.

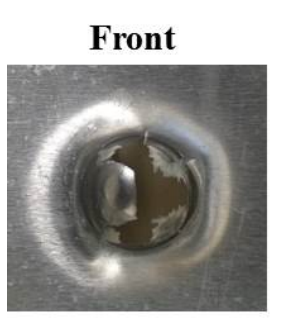

(a)
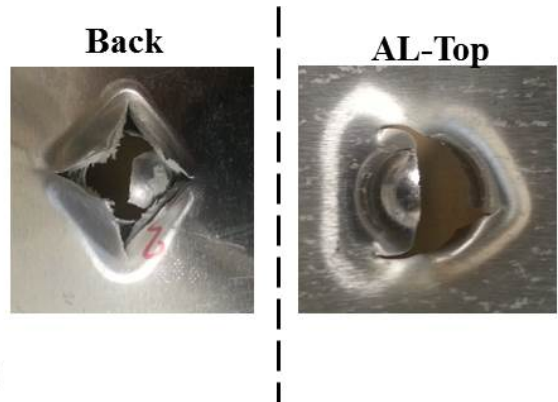

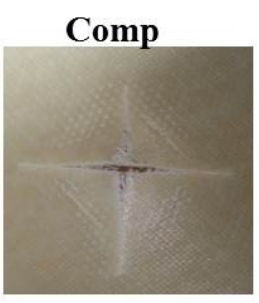

(b)

Figure 5.32: Experimental indentation damage surface. (a) Self and (b) Pseudobonded sample

DD damage of pseudo-bonded sample developed in the form of mutually perpendicular fibre crack as shown in Figure 5.32(b). The above pattern of damage resulted in sub-surface air gap forming exactly under the contact area where fibres crack of both weft and warp direction intersected. With no constraint to the in-plane shear deformation, the generated airgap was soon filled by the outer aluminium layers and eventually formed a thick monolithic region under the contact point. As a result, post DD response of the pseudo-bonded 2/1 panel was driven again only by metal layers and the final failure was governed by a spontaneous cracking of metal layers with no distinction between First Crack (FC) and Through The Thickness (TTT) damage. Similar damage surfaces on top and bottom aluminium layer in Figure 5.32 (b) evidenced this failure process where both layers were cracked via dishing. With a complete loss in flexural integrity and negligible contribution of the GFRP layer, pseudo-bonded sample completely loses its energy absorbing capability where its perforation energy level $\left(E_{T T T}\right)_{\text {pseudo }}$ is observed to be marginally lower compared to self-bonded sample $\left(E_{T T T}\right)_{\text {self }}$.

Damage progression of self-bonded sample was stepwise, starting with FC failure associated with petaling failure of back aluminium layer and followed by TTT failure governed by cracking of front aluminium layer via dishing as illustrated in Figure $5.32(\mathrm{a})$. 


\subsubsection{Low velocity impact response}

Impact response of self and pseudo-bond FML-2/1 to different impact velocity is shown in Figure 5.33. Unlike QSI response, the initial contact slope of both samples are similar for all tested velocities. At lower impact velocity of $V_{i}=1.75 \mathrm{~m} / \mathrm{s}$ (equivalent energy level that induce DD damage by QSI load), both samples experienced a small drop as shown in Figure 5.33(a) which is believed to be DD failure. Corresponding damage surfaces illustrated in Figure 5.34 confirmed this speculation where there are no visible damage on neither of the samples.

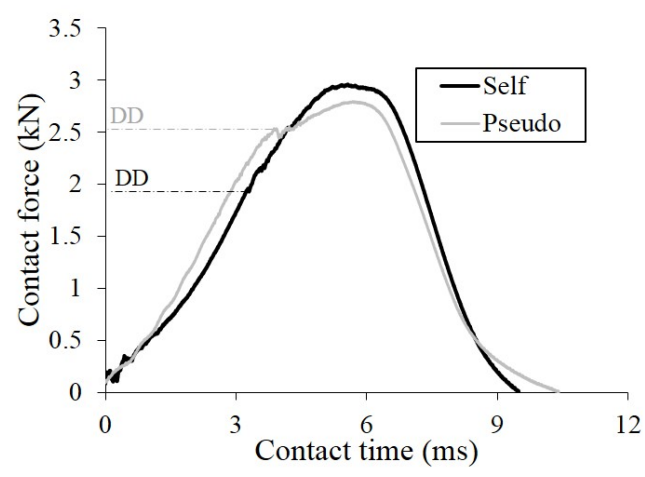

(a)

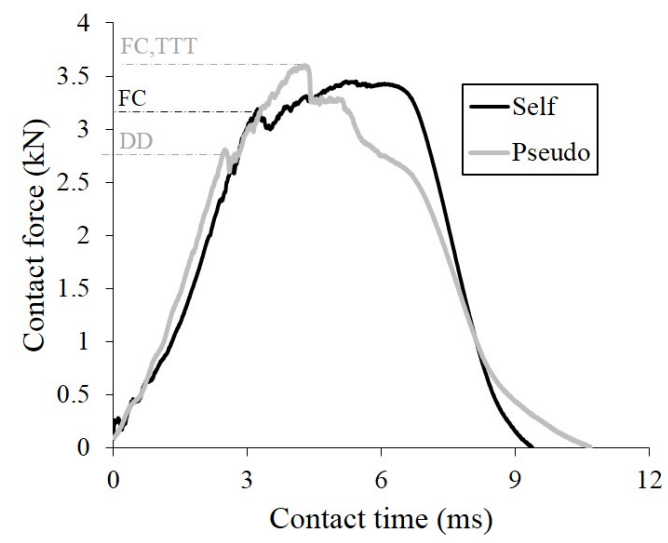

(c)

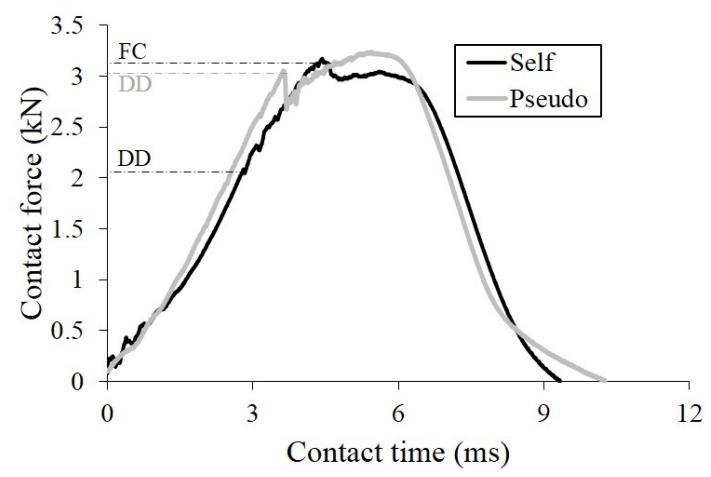

(b)

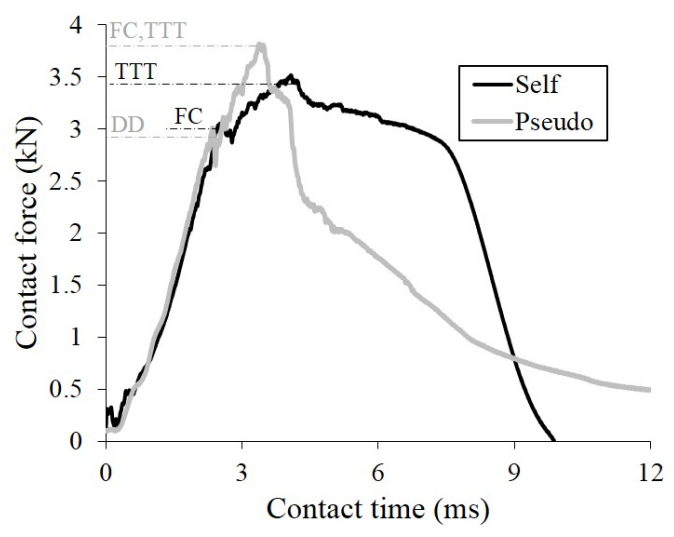

(d)

Figure 5.33: Impact response of self and pseudo-bonded FML-2/1 panel. $V_{i}=1.75 \mathrm{~m} / \mathrm{s}(\mathrm{b}) V_{i}=2 \mathrm{~m} / \mathrm{s}(\mathrm{c}) V_{i}=2.5 \mathrm{~m} / \mathrm{s}$ and $(\mathrm{d}) V_{i}=3 \mathrm{~m} / \mathrm{s}$

It can be noted that the force level to induce DD damage is larger for pseudobonded sample and this scenario is consistent with what has been observed on the impact response of soft side impacted FML-1/1 panel. At slightly larger impact velocity of $V_{i}=2 \mathrm{~m} / \mathrm{s}$, both DD and $\mathrm{FC}$ failure modes appeared on self-bonded sample but only DD damage in pseudo-bonded sample as confirmed in corresponding impact 


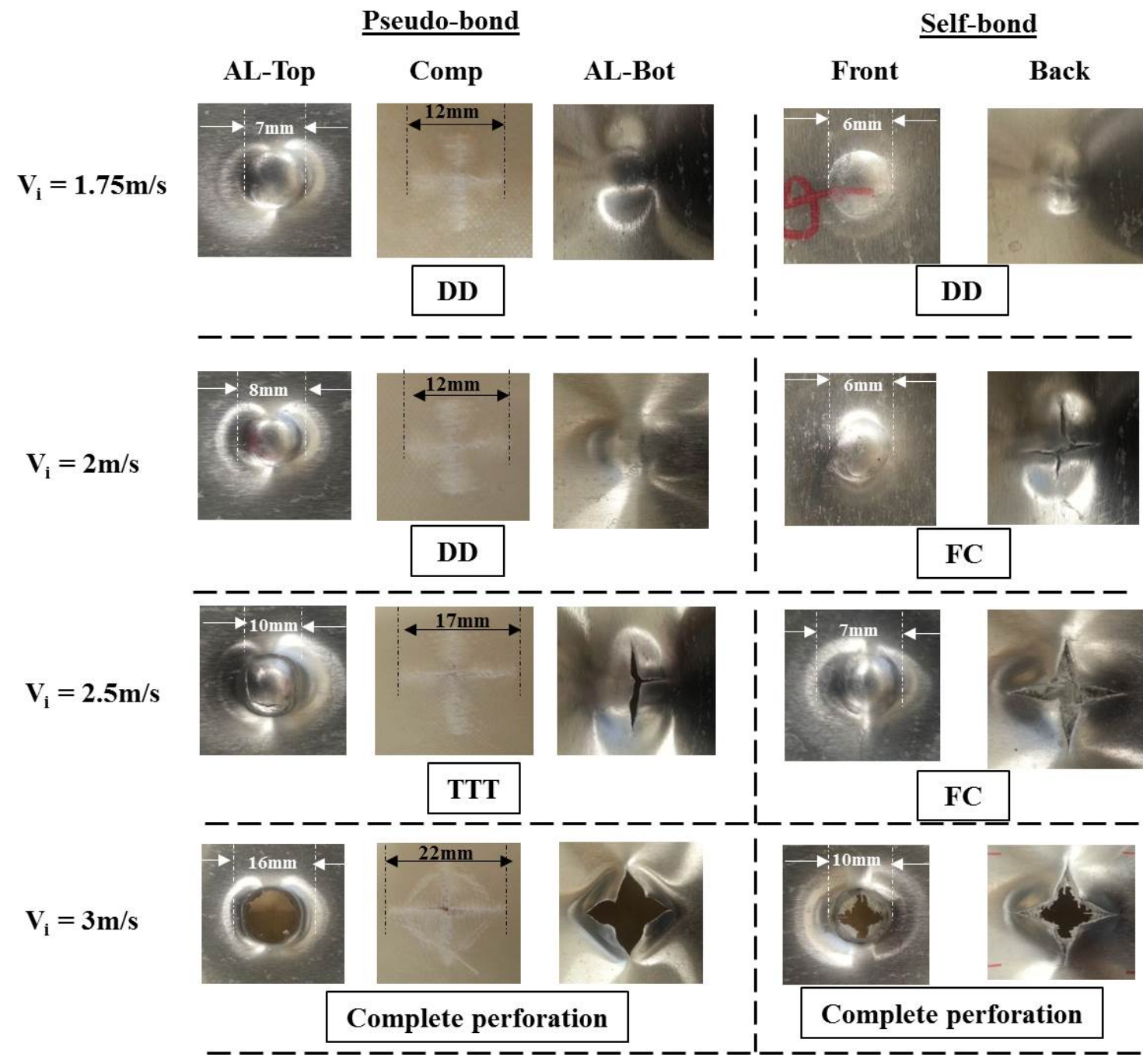

Figure 5.34: Damage surface of impacted self and pseudo-bonded FML-2/1 panel. (a) $V_{i}=1.75 \mathrm{~m} / \mathrm{s}$ (b) $V_{i}=2 \mathrm{~m} / \mathrm{s} \mathrm{(c)} V_{i}=2.5 \mathrm{~m} / \mathrm{s}$ and (d) $V_{i}=3 \mathrm{~m} / \mathrm{s}$ 
damage surface. With further larger impact velocity of $V_{i}=2.5 \mathrm{~m} / \mathrm{s}$, pseudo-bonded sample experienced a penetrated TTT damage whereas only FC damage is obvious in self-bonded sample. At impact velocity of $V_{i}=3 \mathrm{~m} / \mathrm{s}$, both samples underwent complete perforation and their failure shapes are almost similar in shape.

Table 5.9: Numerical predictions of different modes of energy dissipation

\begin{tabular}{cccc|ccc}
\hline \multirow{2}{*}{$V_{i}$} & \multicolumn{3}{c|}{ Self } & \multicolumn{3}{c}{ Pseudo } \\
\cline { 2 - 7 } & $E_{i}(\mathrm{~J})$ & $E_{a}(\mathrm{~J})$ & \% absorbed & $E_{i}$ & $E_{a}$ & $\%$ absorbed \\
\hline 1.75 & 9.13 & 7.28 & 79.77 & 9.32 & 7.44 & 79.81 \\
2 & 11.89 & 9.85 & 82.78 & 11.93 & 9.96 & 83.48 \\
2.5 & 18.40 & 16.05 & 87.21 & 18.51 & 16.45 & 88.86 \\
3 & 26.28 & 24.39 & 92.84 & 26.57 & 26.12 & 98.25 \\
\hline
\end{tabular}

As can be seen in 5.34, a larger contact radius of pseudo-bonded sample at a given velocity shows the enhanced flexibility nature of these panels due to lack of bonding. Experimentally predicted absorbed energy given in Table 5.9 shows that pseudo-bonded sample absorbs larger percentage of energy than self-bonded samples for all impact velocities. The high absorbed energy means that there is more plastic flow and damage in the FML panel.

\subsection{Summary}

This chapter presented the experimental and numerical study of indentation and impact behaviour of FML-1/1 and FML-2/1 configuration. The effect of geometry (contact surface), material (MCI bonding, MVF) and event (impact velocity) related parameters are investigated. The main conclusions extracted from the investigations are summarized as follows.

\section{Behaviour of FML-1/1 panel}

\section{Influence of initial contact surface}

Despite having larger MCI de-bond surface, threshold force $\left(P_{t h}\right)$ and peak contact force of hard surface contact sample were found to be larger than soft surface contact sample for non-perforated impact response. From the energy perspective, the metal layer plays a significant role in the overall impact response regardless 
of the nature of the contact surface. However, contribution by the GFRP layer is found to be highly dependent on initial contact surface. For hard surface contact sample, metal backing accelerates the contribution of GFRP layer to nearly $50 \%$ but amounts only $30 \%$ for soft surface contact sample due to premature tensile crack failure. Decreasing MVF by stacking a larger fraction of GFRP layers resulted in improved damage tolerance for hard side contact samples but showed insignificant influence for soft side contact samples.

\section{Influence of impact velocity}

For all incident impact velocity, the threshold force $\left(P_{t h}\right)$ at which stiffness degradation occurs remains constant for both soft and hard side contact samples. Throughout the impact velocity range, the threshold force of hard side impact sample is larger than soft side impact sample. However, the peak contact force $\left(P_{\max }\right)$ is almost constant for both soft and hard side impact panels at higher velocity range. In conclusion, hard side impact sample seems to yield better impact resistant FML1/1 material than soft side impact sample. However, a larger de-bond boundary of MCI region in hard side impact sample for a given impact velocity raises a caution on its post impact behaviour.

\section{Influence of MCI bonding}

Depending on the side in which impactor/indenter contact first, the contribution of the MCI bond in FML-1/1 is either advantageous or disadvantageous. In soft side impact samples, the presence of MCI bond decreases the threshold load of selfbonded sample whereas in hard side impact samples, its influence is directly opposite for all impact velocity. At higher impact velocities, the influence of MCI on the peak contact force $\left(P_{\max }\right)$ is insignificant for both self and pseudo- bonded samples.

\section{Behaviour of FML-2/1 panel}

\section{Influence of impact velocity}

The threshold force to cause different failure stages (DD, FC and TTT) in FML2/1 laminate is constant for all impact velocity. Magnitude of threshold force and 
peak contact force predicted from numerical simulation agrees well with experimental measurements. The failure progression predicted by numerical simulation showed that failure is preceded by de-bonding of M-C interface, fibre fracture in composite layer, followed by C-M interface debond, then the back AL layer fracture and finally the front AL layer fracture. The predicted failure sequence is found to be the same for all tested velocities. Locus of de-bond area of C-M interface is found to be larger than M-C interface. Aluminium layer dominates the energy absorption behaviour of FML-2/1 panel where nearly $\sim 60-70 \%$ of incident energy has been absorbed by plastic deformation both in bending and membrane stretching.

\section{Influence of MCI bonding}

An absence of MCI bonding between the layers postponed the initiation of composite and aluminum layer damage of FML. The flexibility of the FML panel was increased due to the lack of MCI bonding, as this allows the stacked dissimilar material layer to deform independently of each other. For the same reason, net energy absorbed by the sample with no MCI bond (pseudo) was always greater than sample having MCI (self) for the given impact velocity.

For all the test variables investigated, QSI tests provided an useful energy range for inducing different failure stages in the LVI tests. The order of damage occurrence in both QSI and LVI are unique in all test cases. These information helps the structural designers to characterize the indentation and impact behaviour of given FML panel through the observed contact force response and final damage shapes. 


\section{Chapter 6}

\section{Structural Integrity of Fibre Metal Laminates}

QSI and LVI studies on FML-1/1 in Chapter 5 exposed the role of metal and FRP layers in a metal bonded interply hybrid composite laminates. In compliance with these findings, this chapter investigates the behaviour of woven fabric based FML of different stacking configuration subjected to same loading conditions. Ply clustering is taken as a key variable to investigate the FML panels with similar geometry thickness and metal volume fraction. Experimental and numerical tests are carried to identify different failure modes, force-displacement and energy characteristic curves. Details of test configurations are described in Section 6.2. Investigatory reports of observed QSI and LVI behaviour are discussed in Section 6.3 and 6.4 respectively.

\subsection{Introduction}

In the concept of interply hybridization, extent of plastic deformation and perforation resistance of hybrid laminates were found to be dependent on stacking sequence of dissimilar material layers [6]. The same conclusion even hold for metal bonded FRP structures as shown in the previous Chapter 5. Out of possible modification strategies in FML configuration, optimum impact property for commercially available GLARE grade was achieved based on glass fibre orientation in FRP layer. However, no recommendations were provided on the optimum number of metal and 
composite layer to be used for a given laminate thickness and MVF (so called effect of ply clustering in composite material terminology) to achieve optimum impact response apart from a small hint about necessity of thin metal layers to limit MCI damage by reducing the adhesive shear stress in FML configuration [12].

Knowledge from interply hybrid FRP laminate highlighted that perforation resistance [140], extent of interlaminar delamination [141], flexural ability and capability of energy dissipation by plastic deformation $[6]$ are greatly altered by the difference in the level of ply clustering under equal impact conditions.

Over the years, multiple studies have been carried out to investigate the impact behaviour of GLARE FML panels of different stacking configuration; 2/1 13, 34, 49, $3 / 2[13,20,22,25,35,38,52], 4 / 3[20,35]$ and $5 / 4[20]$. Results of the above mentioned stacking arrangements were also compared $13,20,35,45$ and it was reported that perforation resistance has been increased significantly by stacking more alternate metal and composite layers. However in all the tested configurations, the laminate thickness and MVF were not maintained constant and thus raises a hypothetical situation on whether such improvement was attributed either due to thickness increment or due to stacking sequence or change in MVF.

To guide the designers for optimum selection of number of metal and composite layers for a given FML laminate thickness, it is therefore of interest in this chapter to investigate the effect of ply clustering to quasi-static indentation and low velocity impact behaviour.

\subsection{Test configuration}

As shown in Figure 6.1, three different stacking sequences of constant thick and constant MVF GLARE panels constituting AL-2024-O Clad aluminium and 7781/L530 plain weave glass/epoxy composite prepreg material layers were fabricated by varying the thickness of corresponding material layers i.e. by controlling the level of ply clustering; GLARE - 2/1 (high), 3/2 (medium) and 4/3 (low). A total of six samples were fabricated for each configuration where three of them are for 
QSI test and the remainder for LVI impact test. The geometric details of the fabricated samples are listed in Table 6.1. As can be seen, the measured laminate thickness of $3 / 2$ and $4 / 3$ panels was slightly higher than expected thickness of $3 \mathrm{~mm}$ due to inadequate draining of excess resin in the manufacturing process because of obstruction caused by more number of internal metal layers.

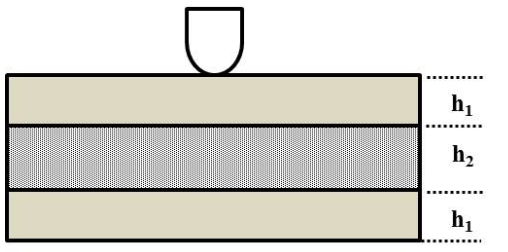

$h_{1}=h_{2}=1 \mathrm{~mm}$

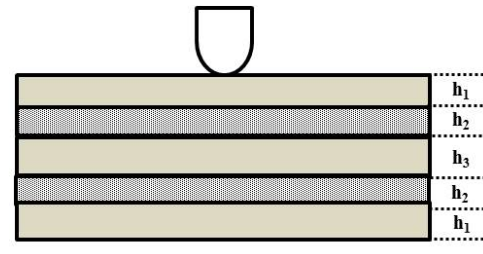

$h_{1}=h_{2}=0.5 \mathrm{~mm}$ $\mathbf{h}_{3}=1 \mathrm{~mm}$ GFRP

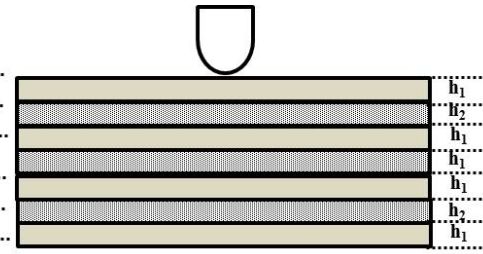

$h_{1}=0.5 \mathrm{~mm} \quad h_{2}=0.25 \mathrm{~mm}$

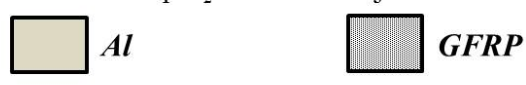

(b)

(c)

Figure 6.1: Stacking geometry of GLARE panels utilized for QSI and LVI experiments; GLARE (a) 2/1 (b) 3/2 (c) 4/3

On the QSI test of three samples, two of them were indented until the contact load drops by $90 \%$ of respective peak value and one was intermittently stopped after the force shows an initial drop in the $P-\delta$ curve. After visual inspection, the samples were manually bisected in order to evaluate the loci of sub-surface damage and MCI de-bonding boundary.

Table 6.1: Experimental structure

\begin{tabular}{ccccc}
\hline Sample & Metal thickness $(\mathrm{mm})$ & Laminate thickness $(\mathrm{mm})$ & MVF & No. of MCI \\
\hline GLARE-2/1 & 2 & 2.93 & 0.683 & 2 \\
GLARE-3/2 & 2 & 3.13 & 0.639 & 4 \\
GLARE-4/3 & 2 & 3.34 & 0.599 & 6 \\
\hline
\end{tabular}

Upon obtaining the approximate baseline energy level to initiate through the thickness $(\mathrm{TTT})$ damage from the quasi-static indentation tests $(\sim 34 J)$, LVI tests were conducted on set of panels at equivalent impact energy range to analyze the effect of the stacking sequence in dynamic loading environment. The incident impact energy was achieved by using an impactor mass of $7.6 \mathrm{~kg}$ with an impact velocity of $3 \mathrm{~m} / \mathrm{s}$. Experimental set up, numerical modeling procedure for QSI and LVI tests are same as that explained in chapter 3 . 
Obtained results from QSI and LVI tests were analyzed based on similar procedure used to investigate FML 2/1 panel in section 5.4 by classifying failure surfaces of FML panel in to four morphologies originally proposed by [20]. Given a correlated numerical results with experimental observations, partition of plastic energy absorbed by each metal layer and extent of MCI damage in each MCI interface were extracted from FE simulation to plot the influence of ply clustering on plastic deformation and interfacial damage in FML.

\subsection{QSI test on FML panels}

\subsubsection{Experimental results}

Figure 6.2(a) depicts the $P-\delta$ curves of the quasi-static indented FML panels, included on the curves are the loci of stages of distinct failure stages observed during experiments; DD, FC and TTT. The slope of initial linear region of $P-\delta$ curve is constant among all panels and suggests that the global flexural stiffness of the FML is independent of the stacking sequence.

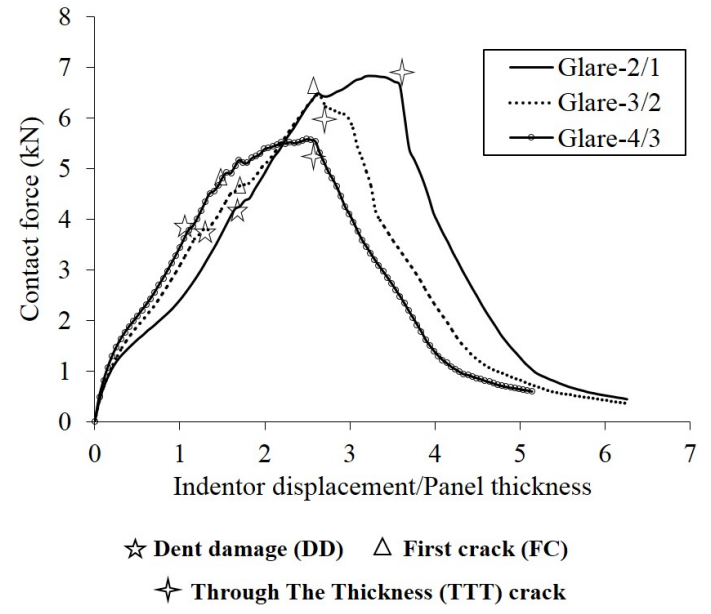

(a)

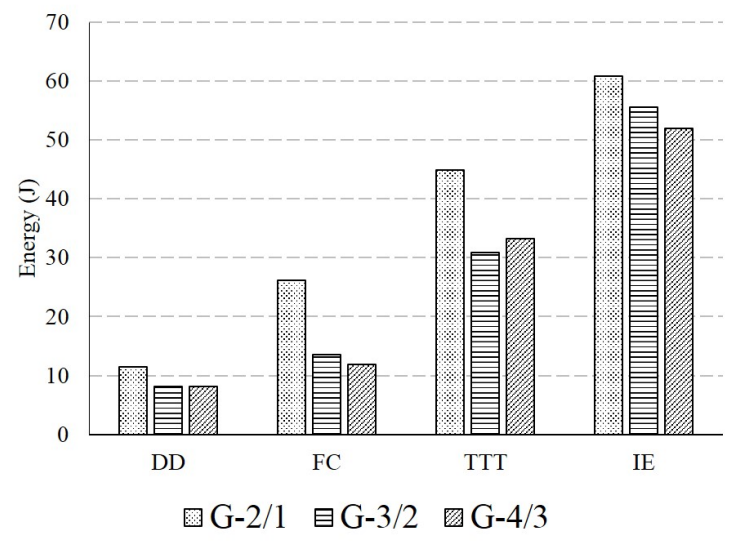

(b)

Figure 6.2: Experimental results from QSI tests. (a) Load-deflection curve, (b) Energy absorbed at different damage level

Following that, the difference in slope of $P-\delta$ curves exists and dictates a clear change in flexural stiffness and it is more pronounced for 2/1 FML followed by $3 / 2$ and $4 / 3$ specimens. This may be due to the difference in the degree of 
plastic deformation of stacked metal layer of different thickness in FML samples as confirmed via simple standard tensile tests on metal sheet with thicknesses of $0.5 \mathrm{~mm}$ and $1 \mathrm{~mm}$ discussed in section 3.3.2. It can be seen that although the ultimate strength ( $190 \mathrm{MPa}$ ) and yield strength ( $50 \mathrm{MPa}$ ) are approximately same for both thickness, the failure strain of thick sheet $\left(\varepsilon_{f}=0.22\right)$ is $70 \%$ larger than thin sheet $\left(\varepsilon_{f}=0.13\right)$. This implies that thicker metal sheets undergo larger plastic deformation compared to thinner metal sheets as reflected in the plastic behaviour of $2 / 1$ indented panels.

The order of failure stage occurrence is confirmed through video monitoring and post-mortem inspections of the specimen at intermittent stops. Apart from extensive plastic flow governed by thick metal layers, it also delayed the threshold limit of all three failure stages as can be seen in Figure 6.2(a). The internal strain energy levels to attain each failure modes are obtained through numerical integration of $P-\delta$ curve using trapezoidal rule:

$$
\int_{\delta_{i}}^{\delta_{i+1}} P(\delta) \mathrm{d} \delta \approx\left(\delta_{i+1}-\delta_{i}\right)\left(\frac{P\left(\delta_{i+1}\right)-P\left(\delta_{i}\right)}{2}\right)
$$

where $\delta_{i, i+1}$ and $P\left(\delta_{i, i+1}\right)$ are the subsequent impactor displacement and corresponding contact load magnitude respectively. The results of this computation are shown in the form of a bar chart in Figure 6.2(b).

Table 6.2: Energy characteristics of indented samples [the values in the bracket () denotes percentage of energy required to initiate current failure stage with respect to previous failure stage]

\begin{tabular}{lllllc}
\hline Sample & $\mathrm{E}_{\mathrm{DD}}(\mathrm{J})$ & $\mathrm{E}_{\mathrm{FC}}(\mathrm{J})$ & $\mathrm{E}_{\mathrm{TTT}}(\mathrm{J})$ & $\mathrm{E}_{\text {net }}(\mathrm{J})$ & $\mathrm{E}_{\mathrm{Per}}=\mathrm{E}_{\text {net }}-\mathrm{E}_{\mathrm{TTT}}(\mathrm{J})$ \\
\hline $2 / 1$ & \multirow{2}{*}{11.41} & $\begin{array}{l}26.15 \\
(129 \%)\end{array}$ & $\begin{array}{l}44.92 \\
(72 \%)\end{array}$ & $\begin{array}{l}60.81 \\
(35 \%)\end{array}$ & 15.89 \\
\hline $3 / 2$ & \multirow{2}{*}{8.12} & $\begin{array}{l}13.53 \\
(67 \%)\end{array}$ & $\begin{array}{l}30.84 \\
(128 \%)\end{array}$ & $\begin{array}{l}55.62 \\
(80 \%)\end{array}$ & 24.78 \\
\hline $4 / 3$ & 8.06 & $\begin{array}{l}11.84 \\
(47 \%)\end{array}$ & $\begin{array}{l}33.18 \\
(180 \%)\end{array}$ & $\begin{array}{l}51.95 \\
(57 \%)\end{array}$ & 18.77 \\
\hline \hline
\end{tabular}

Table 6.2 summarized the absorbed energy magnitude to nucleate appropriate failure stages and also compared percentage of energy absorbed between consecutive 
failure stages. The energy absorbed to initiate dent damage $\left(E_{D D}\right)$ and subsequent first visible crack $\left(E_{F C}\right)$ is found to decrease with the decrease in ply clustering. On the other hand, the subsequent percentage energy required to perforate the specimen $\left(E_{T T T}\right)$ from first crack $\left(E_{F C}\right)$ is found increases. This indicates that ply clustering seems favourable for DD and FC but unfavourable for TTT failure stages. In other words, ply clustering dictates direct proportionality to initiate damage resistance and inverse proportionality to damage tolerance of FML panels.

Moreover, one should note that the post perforation energy (energy absorbed after TTT crack) $\left(E_{P e r}\right)$ is found to be larger for $3 / 2$ configuration and this could be probably due to less influence of the bending stress on the metal layers placed near the neutral axis of panels. As can be seen in Table 2, the tolerance level of $80 \%$ was recorded for $3 / 2$ which has thick metal layer along the neutral axis followed by $57 \%$ for $4 / 3$ which has two thin metal layer close to the neutral axis and only $35 \%$ for $2 / 1$ panels where no metal layers are around the neutral axis.

\subsubsection{Numerical results}

Predicted data from FE simulation are compared with recorded experimental force $(P)$ - displacement $(\delta)$ curves as depicted in Figure 6.3. Predicted maximum force $\left(P_{\max }\right)$ and force magnitudes at each failure stages are correlated well with experiment results. These satisfactory predictions provide a confidence for further in-depth layer wise energy characterization of each stacked layers in FML to address the effect of ply-clustering on the indentation behaviour.

As highlighted earlier in Section 5.4.1 regarding the dominancy of plastic energy dissipation of metal layers on impact behaviour of FML, the quantity of plastic energy $\left(E_{P L}\right)$ stored in each discretized element defining metal layer is computed using the formulation 142 ;

$$
E_{P L}=\int_{0}^{t}\left(\int_{V} \sigma^{c} \dot{\varepsilon}^{P L} d V\right) d \tau
$$

where $E_{P L}$ is the plastic energy stored in volume $V, \sigma^{c}$ is the stress tensor derived 
$\downarrow$ Dent damage $\triangle$ First crack (FC) $\&$ Through The Thickness (TTT) crack

(a)

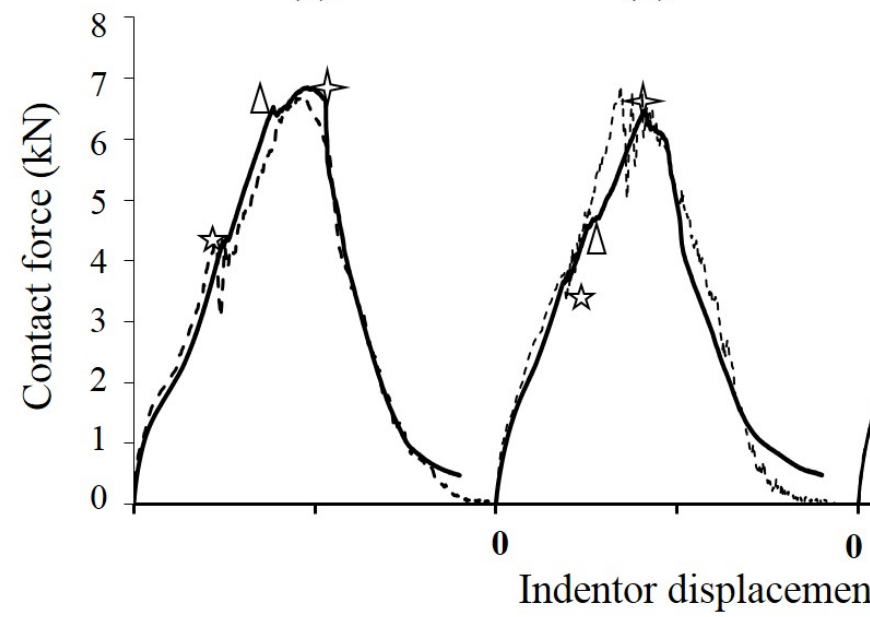

(c)

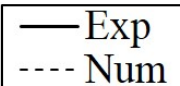

Figure 6.3: Comparison of $P-\delta$ curve from experiment and FE simulation. (a) 2/1 (b) $3 / 2$ (c) $4 / 3$.

based on constitutive material model, $\dot{\varepsilon}^{P L}$ is the plastic strain rate component from total strain rate $\dot{\varepsilon}$ and $t$ is the contact duration of impactor with the panel.

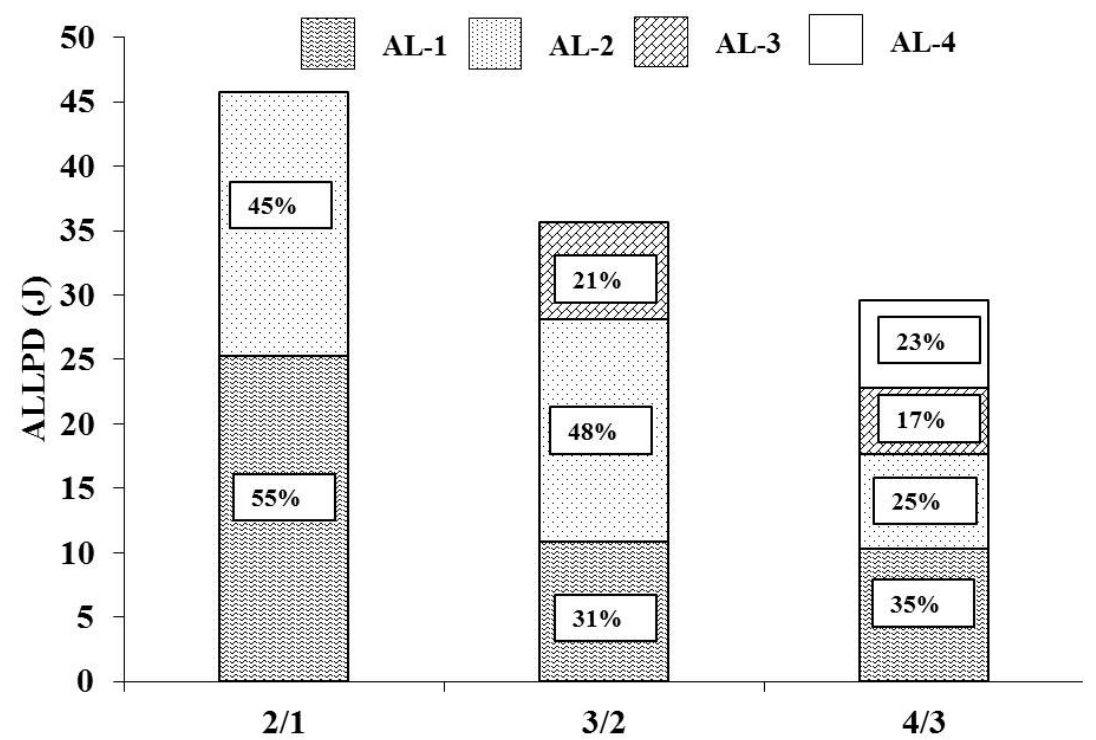

Figure 6.4: Plastic energy absorbed by each aluminium layer of FML in QSI simulation. (Note: In AL-\# ascendingly represents the position of AL layer from front impact face to back non-impact face)

Figure 6.4 compares the predicted percentage of plastically absorbed energy in each metal layer of tested FML configuration. The observation dictates that degree of plastic deformation is strongly dependent on the thickness and on the amount of ply clustering in FML panel. Regardless of location of metal layer and stacking 
configuration of FML panel, thick layer always dissipates larger fraction of plastic energy than thin layers. In general, keeping the laminate thickness and MVF of panel constant, FML panels with more amount of ply clustering absorbs more energy by plastic deformation than panel with distributed layer configuration.

\subsubsection{Damage characterization under QSI loading}

Typical surface and sectional images of indentation damage observed from experiment and numerical simulations are compared in Figure6.5. It can be seen that indentation with a blunt indenter caused a petaling fracture of the back face metal layer and mutually perpendicular fibre fracture in composite layer. The predicted FE failure shapes agreed rather well with the experimental failure modes.
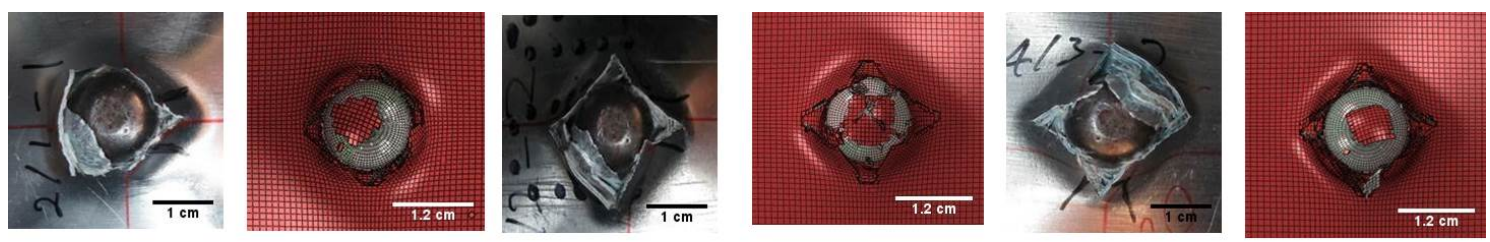

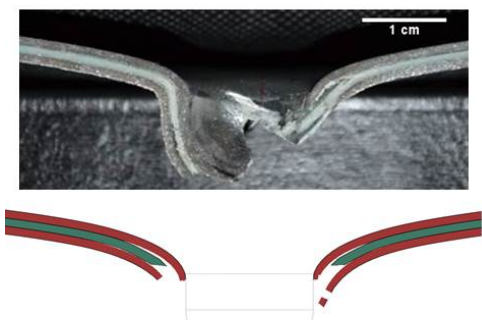

(a)

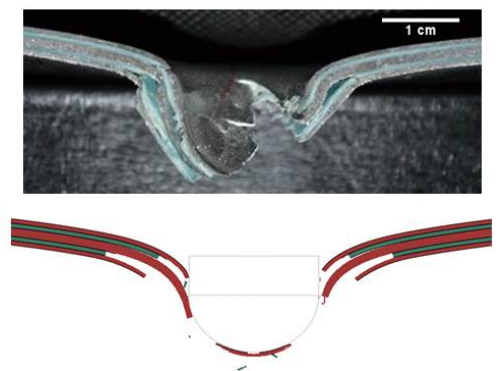

(b)

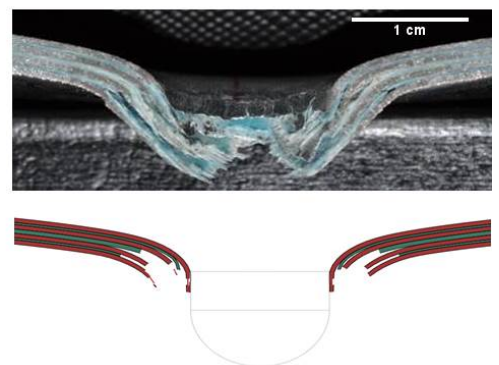

(c)

Figure 6.5: Damage morphology of indented FML Glare panels (a) 2/1 (b) 3/2 (c) 4/3. (First row: Damage shape of back face of panels obtained from experiment and numerical simulation. Second row: cross sectional image from experiment. Third row: Cross sectional image from numerical simulation)

From sectional inspection, the extent of sub-surface damage was observed to be larger than the exposed outer damage surface and the through thickness damage pattern resembles that of a frustum with smaller damage area on front contact surface and extended to larger area as move towards the bottom of the panel. Microscopic investigation revealed no inter-ply delamination between the composite layers but shows apparent traces of MCI de-bonding as shown in Figure 6.6 espe- 
cially MCI interfaces formed by thick metal layers. Probable reasons for this peculiar de-bond behaviour might be multiple as follows; (i) ply grouping decreases interfaces available for de-bonding and in turn increases the de-bonding size in available interfaces [140], (ii) Clustering of plies causes greater stress concentration at the interfaces due to larger variations of bending stiffness between dissimilar layers [141], and (iii) Thicker adjacent dissimilar plies always induce larger shear stress along interface region compared to thinner plies [12].
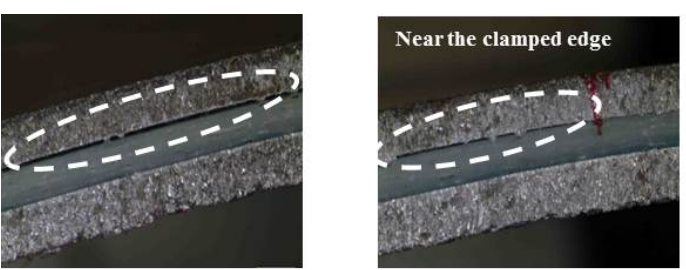

(a)

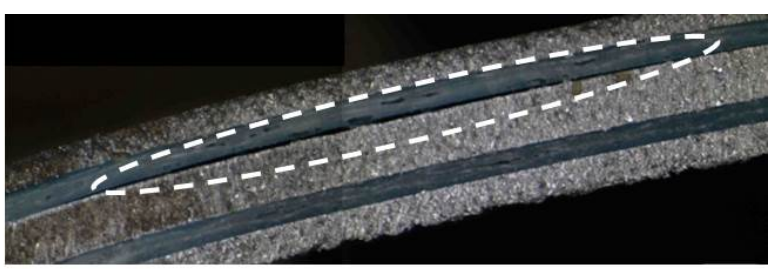

(b)

Figure 6.6: Microscopic images illustrating de-bonding of MCI (a) 2/1 (b) 3/2.

As revealed from the indentation behaviour of FML 1/1 described in section 5.4.1. that the damage extension of MCI is dependent on whether the initial contact surface is soft or hard side. It can be possible to classify MCI of FML in to two distinct region with respect to the indentation direction (see Figure 5.28 in Chapter 5); metal-composite (M-C) and composite-metal (C-M) interface. De-bond boundary contours of each MCI regions predicted by cohesive element definitions are shown in Figure 6.7. Based on the aforementioned type of MCI interface, the following important points are drawn:

- Regardless of the location of MCI layer in the panel, M-C interface degraded earlier in all the tested configurations and this behaviour is likely due to severe strain incompatibility between top metal and bottom composite layer [35].

- The damage shape is doubly symmetrical either in the shape of a rhombus (in M-C) or a circle (in C-M) because of prevailing perpendicular plane of material symmetry. Comparing these with previous predictions for $1 / 1$ panels 143 , the extent of M-C de-bond interface is found to be critically dependent on the boundary of adjacent composite layer failure. 
- The M-C interface at any location in the panel has a smaller damage area compared to its subsequent C-M interface, and they increased in size towards the back face. With the increase in hindrance to plastic flow, the average de-bond area $\left(A_{d}\right)$ on $3 / 2$ and $4 / 3$ panels was observed to be $20 \%$ and $30 \%$ respectively smaller than that of the $2 / 1$ panel.

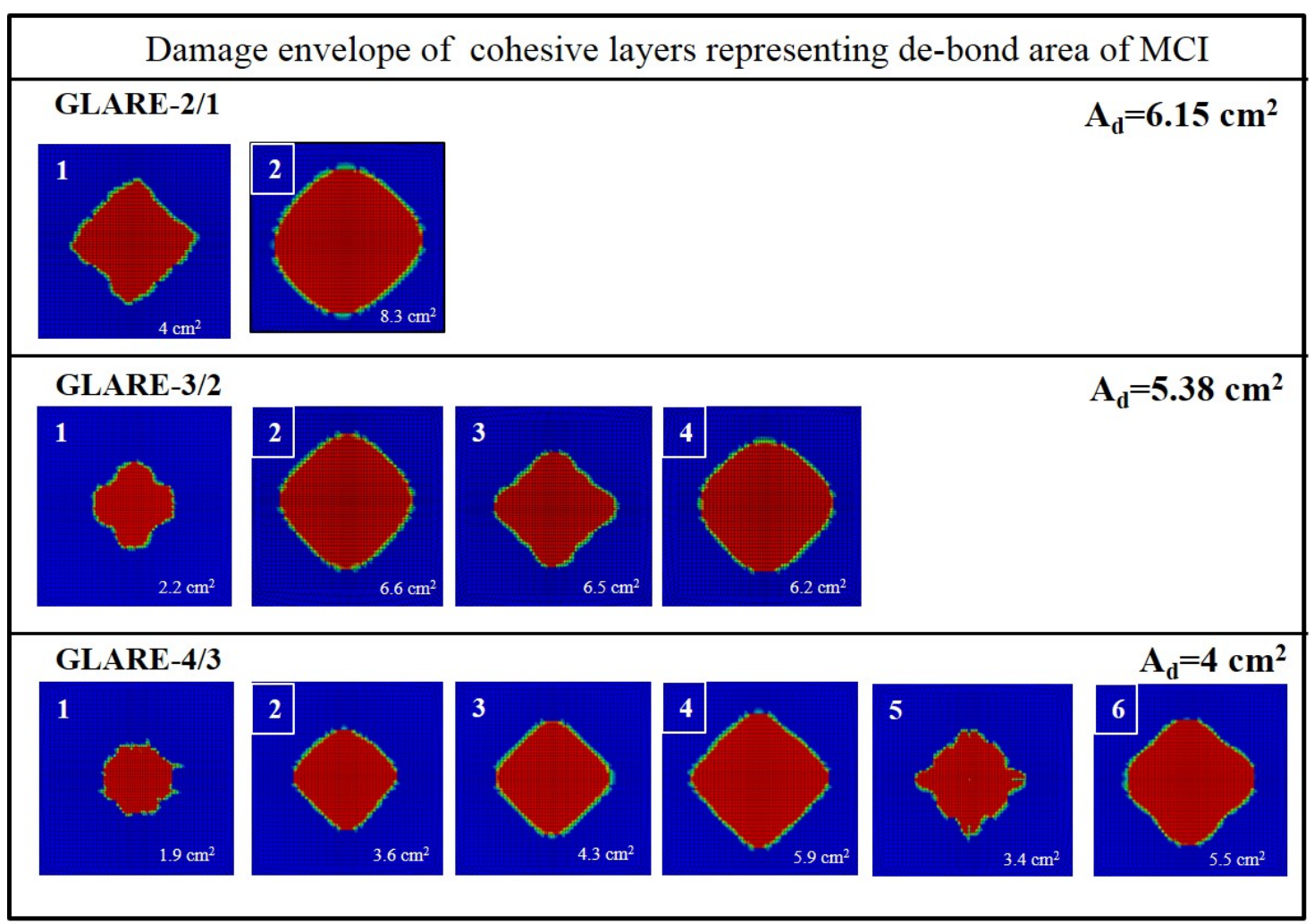

\# $\quad \mathrm{A}_{\mathrm{d}}=$ Average de-bond area.

* Numbers in the top left corner represents MCI layer number in a given ply. (without outline: $\mathrm{M}-\mathrm{C}$ interface; With outline: C-M interface.

** Readings in the bottom right corner represents the de-bond area of each MCI layer (Note: For comparison, Maximum contact area is $1.35 \mathrm{~cm}^{2}$.)

Figure 6.7: Numerical predictions of MCI de-bond boundaries by cohesive elements

\subsection{LVI test on FML panels}

\subsubsection{Experimental and Numerical results}

Unlike QSI tests, experimental characteristic curves of LVI tests do not provide much substantial insight information about impact failure stages apart from few characteristic parameter like peak force and the amount of energy absorbed during impact. Thus, experimental and numerical observations are analyzed in parallel to 
draw out the influence of ply clustering to impact load. Figure 6.8 compares the force $(P)$ - displacement $(\delta)$ curves of experiment and numerical LVI tests. Overall, the agreement between them is excellent and the numerical simulations are well capable to inscribe the load values of distinct failure stages.

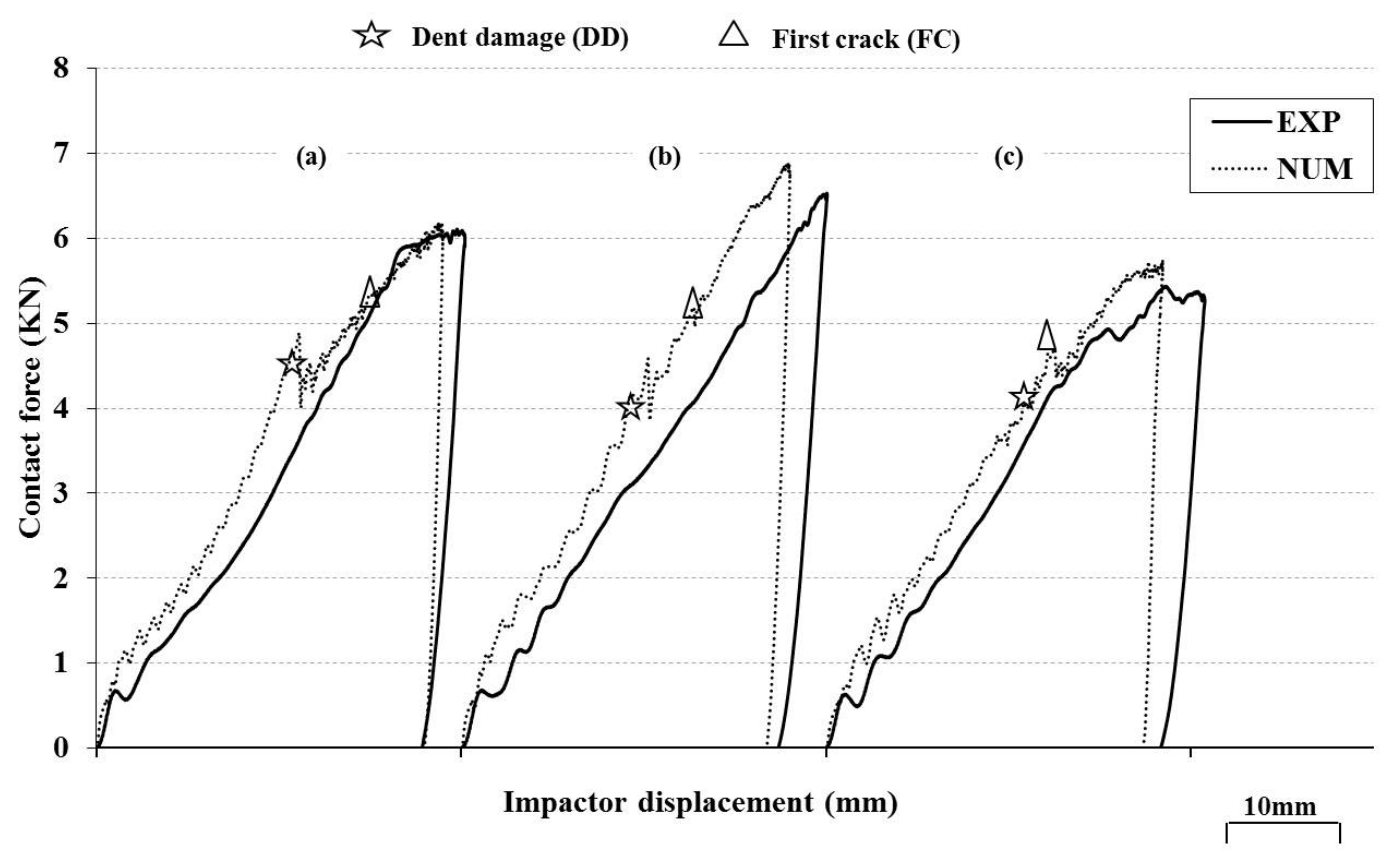

Figure 6.8: Contact load-impactor displacement curves for (a) 2/1 (b) 3/2 (c) 4/3

An interesting observation is that, threshold load required to nucleate DD damage reads approximately $4 \mathrm{kN}$ for all tested configurations and also found to be marginally same for both QSI and LVI tests. This dictates that, the failure initiation load is independent of the ply clustering and unaffected by the rate of loading.

A summary of important impact characteristic parameters from experimental tests are tabulated in Table 6.3. Similar to QSI response, the $P_{\max }$ of $4 / 3$ panel is smaller and highlight its inferior impact resistance among tested configurations. In contrary to the difference in energy absorption characteristics with respect to level of ply clustering in QSI test, net energy absorbed $\left(E_{a}\right)$ and energy dissipated by plastic deformation of metal layer (see Figure 6.9) is observed to be almost constant for impacted FML samples. However, the difference in plastic energy absorption level with respect to metal sheet thickness in LVI follows approximately same scenario as that of QSI tests as illustrated in Figure 6.4. 


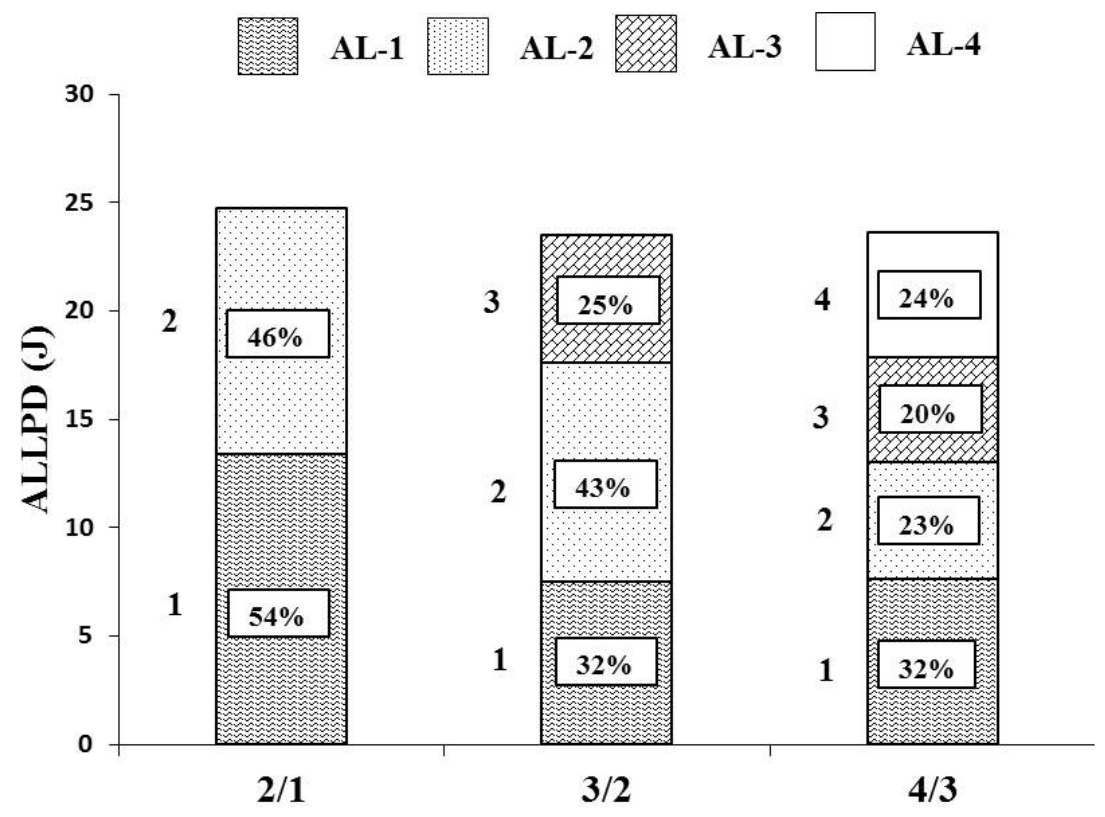

Figure 6.9: Plastic energy absorbed by each aluminium layer of FML in LVI simulation

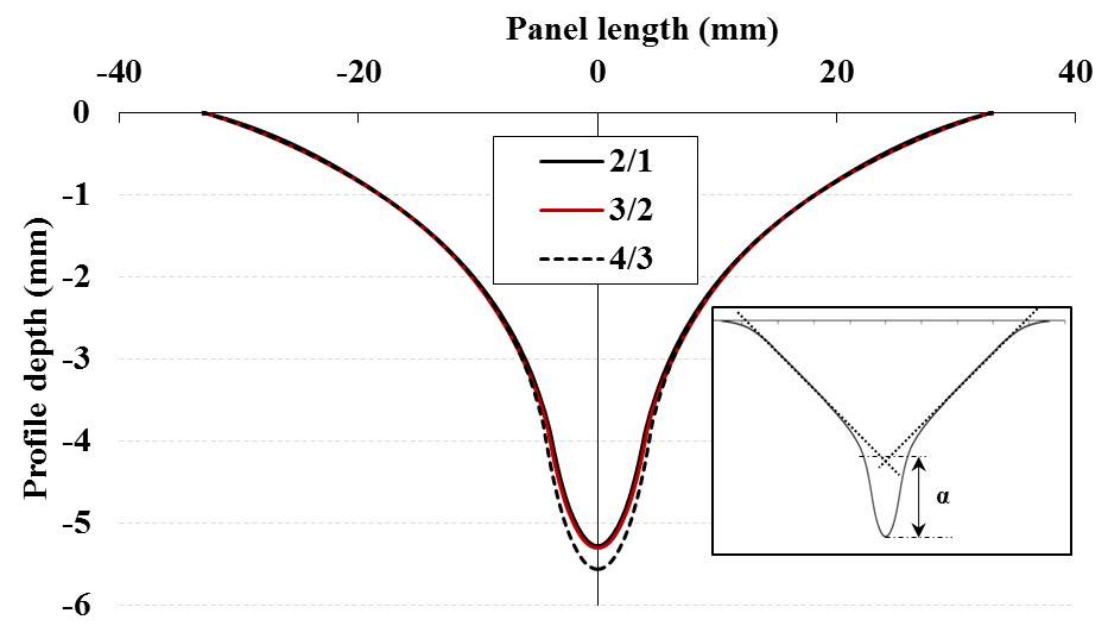

Figure 6.10: Post impact Deflection profile of FML panel

As can be seen from the post impact deflection contours shown in Figure 6.10, there is no significant difference in the deflection profile between FML panels of different stacking configurations. Despite the fact that all the panels have approximately the same constant maximum impactor displacement $\left(\delta_{\max }\right)$, panel $4 / 3$ underwent larger residual $\left(\delta_{r}\right)$ deformation (see Table 6.3) under the contact region and with intensive damage beneath its contact area. Furthermore, the larger local indentation depth $(\alpha)$ derived from the deflection profile also reinforces the argument of deprived impact response of less ply clustered 4/3 FML panel. 
Table 6.3: Average characteristic parameters of FML panels subjected to impact energy $\left(\mathrm{E}_{\mathrm{i}}\right)$ of $34 \mathrm{~J}$

\begin{tabular}{ccccccl}
\hline $\begin{array}{c}\text { Sam- } \\
\text { ple }\end{array}$ & $\begin{array}{c}\mathrm{P}_{\max } \\
(\mathrm{KN})\end{array}$ & $\mathrm{E}_{\mathrm{a}}(\mathrm{J})$ & $\delta_{\max }(\mathrm{mm})$ & $\delta_{r}(\mathrm{~mm})$ & $\alpha(\mathrm{mm})$ & Observations \\
\hline $2 / 1$ & 6.12 & 31.17 & 10.06 & 8.88 & 3.2 & $\begin{array}{l}\text { Arbitrary shaped crack on back face } \\
3 / 2\end{array}$ \\
6.44 & 30.62 & 10.14 & 8.85 & 3.2 & $\begin{array}{l}\text { Star shaped crack (4 petals) on } \\
\text { back face }\end{array}$ \\
$4 / 3$ & 5.47 & 31.32 & 10.29 & 9.05 & $3.7 \begin{array}{l}\text { Star shaped crack (4 petals) on } \\
\text { back face }\end{array}$ \\
\hline
\end{tabular}

Note: $\mathrm{P}_{\max }=$ Maximum contact force, $\mathrm{E}_{\mathrm{a}}=$ Absorbed impact energy, $\delta_{\max }=$ Maximum displacement, $\delta_{r}=$ Residual displacement, $\alpha=$ Local indentation.

\subsubsection{Damage characterization under LVI loading}

A typical external damage surface of impact loaded FML panel and corresponding bisected cross-sectional views are shown in Figure 6.11. It can be seen that none of the impacted panel had TTT crack and all the panels showed only FC and DD failure stages. However there exists a difference in the number of metal layers fractured for a given impact energy of 34J; 1 out of 2,1 out of 3 and 3 out of 4 for $2 / 1,3 / 2$ and $4 / 3$ panel respectively. In specific, stacking thick metal layer near the middle surface resulted in better damage resistance for $3 / 2$ panel and as a result this panel exhibit highest $P_{\max }$ magnitude. As can be seen in Figure 6.11(c), only the bottom most metal layer is fractured for the $3 / 2$ panel.

Except the uppermost MCI interface of $2 / 1$ panel where de-bonding is macroscopically visible, no other widespread de-bond region has been observed in the bisected panels shown in Figure 6.11(b). The discrepancy between permanent deformation contour of plastic metal layer and straight contour of elastic composite layer cause excessive peel stress concentration along the interfacial region during the rebound phase of non-perforated LVI event as illustrated schematically in Figure 5.18. The peel stress concentration increases with increase in ply clustering due to large mismatch in bending stiffness between dissimilar material layers which may be the reason for the weird de-bond behaviour in clustered $2 / 1$ panel. Other interfaces show dis-continuous de-bond distributions which are visible only under the microscope and however they are extremely difficult to quantify due to thinning of one material layer with other under the contact area. With the help of the finite 
element results, the de-bond nature of MCI interfaces are apparently predicted (see row 3 of Figure 6.11(b)). Unlike MCI distribution of QSI tested panels, the difference in de-bond area between the $\mathrm{M}-\mathrm{C}$ and $\mathrm{C}-\mathrm{M}$ interface are rather insignificant in impacted panels. Nevertheless, the average de-bond area is found to decrease with decrease in ply clustering.

(a)

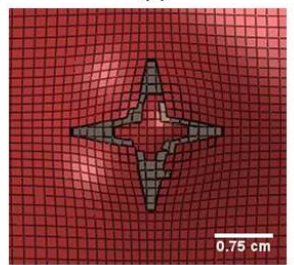

(b)

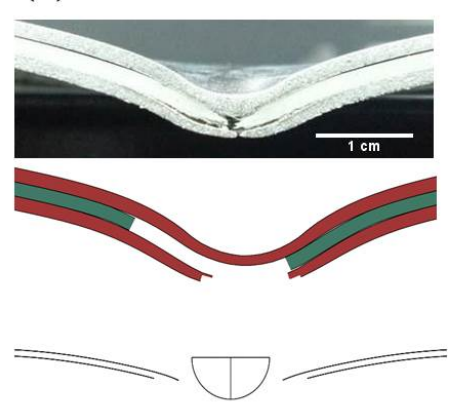

(i) (ii)

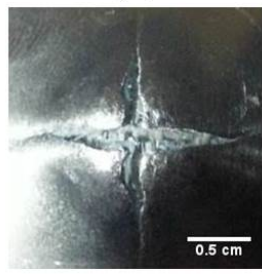

(c)

(i)

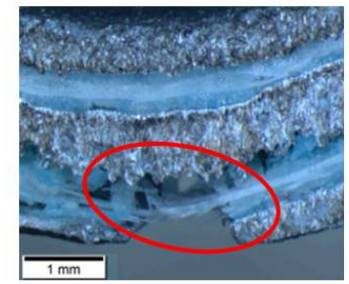

(ii)

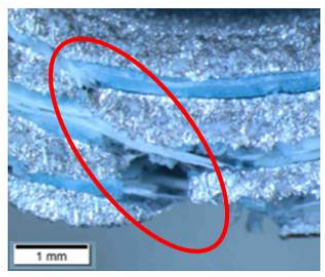

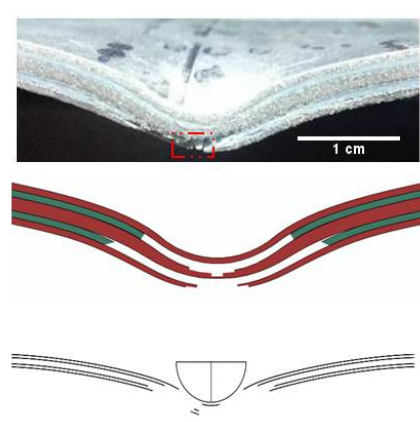

(ii)

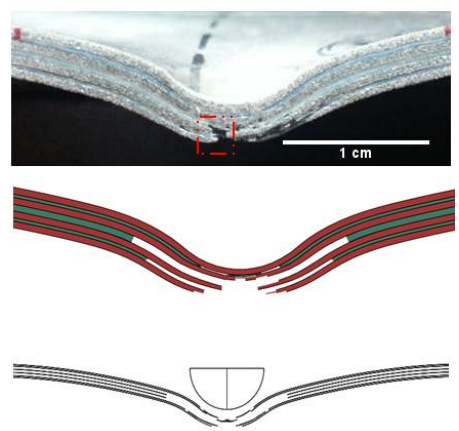

(iii)

Figure 6.11: Damage morphology on LVI panel (a) Typical shape on non-impact side (i) Experimental (ii) Numerical. (b) Cross sectional view (i) 2/1 (ii) 3/2 (iii) 4/3 [Note: Row 1-cross sectional view -experiment, Row 2-cross sectional view Numerical, Row 3-Detail of MCI de-bond prediction by cohesive elements]. (c) Zoomed view of ply fracture (i) $3 / 2$ (ii) $4 / 3$

\subsection{Influence of ply clustering - an interply hy- bridization concept}

The large deflection response of clamped thin circular plate is dominated by two types of deformation modes under lateral load conditions; flexural deflection and membrane stretching. These deformation modes are highly interactive with each other and resistance to any one mode or both will results in premature failure of panel. Besides, plastic flow tendency and flexural ability of ductile layers had been found to be attenuated by interply hybridization of brittle layer $[6]$. These 
potential arguments provide the reason for inferior load resistances of $4 / 3$ panel in QSI and LVI tests. For the given FML thickness and MVF, decreasing the cluster of metal and composite layers can constrain the membrane stretching of metal layer which in turn increases the panel bending stiffness. Higher bending stiffness causes earlier FC and TTT failure stages and resulted $4 / 3$ panels as a lowest load carrying configuration (lower $P_{\max }$ ) compared to $3 / 2$ and 2/1. Despite larger load carrying capability, highly clustered 2/1 FML panel also carry larger MCI de-bond area both in QSI and LVI test.

Comparisons of plastic energy predictions show that increase in ply clustering will increase the amount of plastic energy absorption in metal layers of FML. About $75 \%$ of internal energy is found to be dissipated by means of plastic deformation on highly clustered $2 / 1$ followed by $64 \%$ on intermittent $3 / 2$ configuration and $57 \%$ on dispersed 4/3 ply configuration (see Figure 6.4) in QSI tests. However for a given impact energy of 34J in LVI tests, the difference in the plastic energy is insignificant (see Figure 6.9). Nevertheless, exposing different number of fractured metal layers among tested configuration (see Figure 6.11), the current deductions are inconclusive and requires that LVI test to be done over the range of impact energy until complete perforation in order to draw out maximum plastic energy dissipation of each ply cluster configuration.

\subsection{Summary}

Influence of ply clustering towards structural integrity of FML panels was investigated by means of $2 / 1,3 / 2$ and $4 / 3$ stacking configurations. Maximum load carrying capability, tendency of plastic flow in each metal layers in the FML and locus of de-bond damage induced along MCI interface were evaluated for QSI and LVI load cases. The former two characteristics had found direct proportionality while the latter MCI de-bond area exposed inverse proportionality to the level of clustering of metal and composite layers in FML. Such responses dictated a fact that "clustering of dissimilar material ply thickness enhances the perforation resistance of a 
FML structure but degraded the prolonged overall integrity of a structure". In other words, ply clustering on FML provide positive synergism to damage resistance but impose negative synergism to damage tolerance. These conclusions could guide the designers to select optimum stack configuration for FML panels of given thickness to meet customer end requirements. However, further research is required to reassess the damage tolerance statement in quantitative terms using post-indent/impact investigation methods like compression after impact (CAI) to reaffirm the afore-stated concluded statement. 


\section{Chapter 7}

\section{Conclusions and future work}

This chapter summarizes the major contributions to the understanding of indentation and low velocity impact behaviour of FML achieved in this research work. Section 7.1 presents the major conclusions of the present study. Future studies related with the current work are recommended in Section 7.2 .

\subsection{Concluding remarks}

The main conclusions that were deduced from this research are:

\section{Robust framework of the finite element model}

A robust and reliable finite element model was developed to simulate the indentation and impact response of FML. The developed finite element model reproduces all the physical conditions of experimental set-up such as influence of clamp fixtures, contact constraints between target-impactor and between target-clamp fixtures. With the aim of attaining realistic predictions and to avoid any numerical issues, distinct computational capabilities provided by ABAQUS software (hourglass control, distortion control, mass scaling) were implemented.

The behaviour of material constituents of FML were defined using appropriate constitutive models to simulate both undamaged and damaged response. Experimental observations showed that the interfacial de-bonding between metal and woven fabric composite layers is the important interlaminar damage found in the woven 
fabric based fibre metal laminates and showed no delamination damage between the woven fabric plies. Hence, the cohesive interface elements were included between metal and composite layers in the finite element model to account for the onset and propagation of the MCI de-bonding damage in FML.

In total, three non-interactive material models were introduced to account for metal, composite and cohesive interface behaviour in the finite element model. The damaged response of each layer was captured using continuum damage mechanics (CDM) based damage initiation criteria and fracture mechanics based damage evolution criteria. 1) Ductile failure criterion for the metal layer, 2) Hashin failure criterion for the composite layer and 3) Quadratic nominal stress criteria for the cohesive MCI layer were used to initiate the failure in each material layers. The reliability of the metal and composite material model was validated separately with the respective observed results of monolithic samples. The optimum mesh density for the simulation of the de-bonding damage of cohesive elements was estimated from the results of the mode-I DCB fracture simulation.

High fidelity and reliable numerical results were obtained with the developed finite model. From the comparison of the predicted results and the damage shapes with those obtained from the experimental observation, the ability of the developed finite element model and the suitability of selected material models were justified. Partitioning of whole internal strain energy of the system into layer-wise and dissipation mode-wise classifications allowed one to understand the role of each material and their corresponding energy dissipation mechanism. The developed finite model also provided valuable information of the MCI interface behaviour which is virtually impossible to assess from experiments.

\section{New knowledge on behavioral response of FML}

By using FML-1/1 configuration, the internal damages induced by the indentation and impact load were easily identified due to the exposed area of each material layer. In the present work, fundamental behavioral responses of FML panels were studied using $1 / 1$ and $2 / 1$ configuration. Structural integrity of FML panels to quasi-static 
indentation loading was investigated using $2 / 1,3 / 2$ and $4 / 3$ stacking configuration.

The conclusions of the new and original findings are:

1. Initial response of the FML-1/1 panel is dependent on the material property of the target surface receiving the impactor.

2. Damage resistance of FML-1/1 is improved when the striker impact on the composite material surface (hard surface impact).

3. Though plastic deformation of metal layer absorbs most of the incident impact energy in FML-1/1, the contribution level and mode of energy dissipation by composite layer is completely different with respect to initial impact surface. For soft surface impact, the contribution towards energy absorption is small and the incident impact energy is dissipated when new tensile crack surfaces are formed while for hard surface impact, the contribution level is larger and the incident impact energy is mainly dissipated by flexural deformation.

4. The failure sequence of the material layers in both QSI and LVI tests are unique. Fracture growth of metal layer always progressed from back (nonimpact) to front (impact) surface. Figure 7.1 shows the predicted failure sequence of the material layers of FML-1/1 and FML-2/1 panel. Common in both panels is that failure is initiated along M-C MCI interface (interface with metal layer on front and composite layer on back) soon after followed by composite failure.

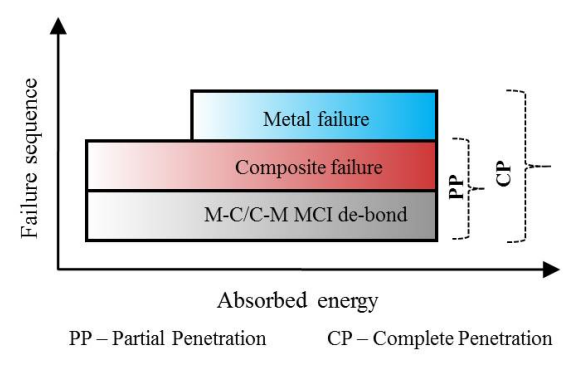

(a)

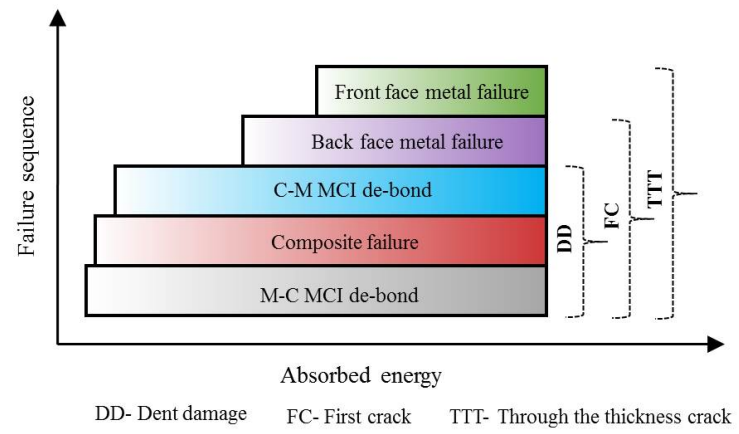

(b)

Figure 7.1: Failure sequence of FML to indentation and impact load. (a) 1/1 and (b) $2 / 1$ panel 
5. The absence of adhesion between metal and composite layer not only delayed but also improved the damage resistance of FML. However, such design may not be acceptable in real load carrying applications due to the lack of its structural stability.

6. Regardless of the initial contact surface and level of bonding between metal and composite layer, the threshold force at which damage initiation occur in FML panel remains constant for the range of the impact velocity considered.

7. For a given panel thickness and metal volume fraction, the amount of clustering between metal and composite layer significantly affected the plastic deformation of FML, the resultant load carrying capability and the extent of damage in each material layers. The clustering level is observed to be directly proportional to the perforation resistance and inversely proportional to the damage tolerance of FML panel. The structural integrity of FML panel was shown to be dependent on the number of metal and composite layer constituted within the given panel thickness.

\subsubsection{Original contributions in this research}

1. A robust and reliable finite element model to simulate indentation and low velocity impact response of woven based FML with a suite of material failure criteria and interface failure criterion is developed.

2. Interfacial de-bonding of MCI is accounted in the developed finite element model using three dimensional cohesive interface elements and their influence to overall behaviour is assessed.

3. The new knowledge of the role of material constituents of FML to indentation and impact load is presented and discussed. This includes elaborate details of the study of structural integrity of different stacking configured FML panels to quasi-static indentation and low velocity impact load. 


\subsection{Suggestions for future work}

Further extensions related to the current scope of research are as follows:

1. Influence of impact (front) face metal layer to the impact response of FML panel is dominant and also crucial. It primarily controls the initial impact response (contact stiffness) and determines the perforation resistance of panel since this is the final layer that will failure due to impact. Having reliable a finite element model to be able to predict a realistic impact response, it would be informative to conduct parametric study to understand the effect of impact face metal layer having different stiffness and strength parameter on the resultant impact response.

2. Current investigation on the quasi-static indentation and low velocity impact behaviour of woven based FML showed no interlaminar delamination between woven fabric plies. It is well known that composite layers of unidirectional fibre (UD) based FML dissipates most of the absorbed impact energy by means of interlaminar delamination. With the availability of a comprehensive literature on unidirectional fibre based FML, it would be interesting to make a comparative study between geometrically equivalent UD based FML with woven based FML and highlight the beneficial aspects of each configuration to structural response.

3. MCI de-bond damage of FML panel showed negligible influence on the experimentally measured characteristics response curves. However, their hidden extended damage region due to impact load might reduce the stability of FML structures before the estimated endurance. Hence, it is essential to investigate the post impact behavioral response of FML panels using tests procedures like compression after impact (CAI).

4. Impact study in the current research showed damage resistance of FML-1/1 is enhanced when the panel was impacted on composite (hard) surface. This 
scenario should provide the strong motivation to conduct the impact survey on FML panels having composite as its outermost layer in future. If the study shows such stacking configuration is feasible and durable, then the resultant FML not only provides enhanced damage resistance but also give inherent corrosion protection to the embedded metal layers.

5. Lack of MCI adhesive bonding between metal and composite layer showed that damage initiation of the composite layer is delayed and also encouraged the metal layer to undergo larger plastic deformation. By investigating FML panels with different level of MCI bond strength, it might be possible to tailor an optimum FML panel with better damage resistance and energy absorption.

6. For a given material and panel thickness, energy-absorbing capability of FML is dependent on the thickness of the metal layers. However, the ductility of metal sheet is inversely proportional to its thickness. In such conditions, geometrically asymmetric hybrid laminates might be the best solution to achieve the required energy absorption level. Hence, the study can be extended to investigate the energy absorbing characteristics of FML panel with geometric asymmetry configurations. Moreover, studies with material asymmetric configurations are also highly encouraged. In specific, it would be interesting to investigate FML panel with very stiff front layer and less stiff bottom layer for better impact resistant and energy absorption capabilities.

\subsection{List of Publications}

\section{Journals}

1. Chai, G.B. and P. Manikandan, Low velocity impact response of fibre-metal laminates A review. Composite Structures, 2014. 107(0): p. 363-381.

2. Manikandan, P. and G.B. Chai, A layer-wise behavioral study of metal based interply hybrid composites under low velocity impact load. Composite Structures, 2014. 117(0): p. 17-31.

3. Manikandan, P. and G.B. Chai, A similitude approach towards the understand- 
ing of low velocity impact characteristics of bi-layered hybrid composite structures. Composite Structures, 2015. 131: p. 183-192.

4. Manikandan, P. and G.B. Chai, Role of material constituents of fibre metal laminates under indentation and impact load. Manuscript in preparation.

5. Manikandan, P. and G.B. Chai, Effect of ply clustering in fibre metal laminates under indentation and impact loading. Manuscript in preparation.

\section{Conference}

1. Manikandan, P. and G.B. Chai. Impact face influence on low velocity impact performance of interply laminated plates.International conference on Experimental Mechanics, 15-17 November 2014, Singapore.

2. Manikandan, P. and G.B. Chai. On suppressing the indentation failure of glassepoxy cross ply laminates by elasto-plastic interleaves. $18^{\text {th }}$ International conference on Composite Structures, 15-18 June 2015, Lisbon, Portugal. 


\section{References}

[1] Serge Abrate. Impact on composite structures. Cambridge University press, 1998.

[2] S.R Reid and G.Zhou. Impact behaviour of fibre-reinforced composite materials and structures. Woodhead Publishing Ltd and CRC Press LLC, 2000.

[3] Fast \#48 Airbus. Flight airworthiness support technology. Airbus Technical Magazine, August 2011.

[4] L. B. Vogelesang and A. Vlot. Development of fibre metal laminates for advanced aerospace structures. Journal of Materials Processing Technology, 103(1):1-5, 2000.

[5] J. K. Kim. Methods for improving impact damage resistance of CFRPs. Key Engineering Materials, 141-143:149-168, 1998.

[6] B. Z. Jang, L. C. Chen, C. Z. Wang, H. T. Lin, and R. H. Zee. Impact resistance and energy absorption mechanisms in hybrid composites. Composites Science and Technology, 34(4):305-335, 1989.

[7] Jang-Kyo Kim and Man-Lung Sham. Impact and delamination failure of woven-fabric composites. Composites Science and Technology, 60(5):745-761, 2000.

[8] J G. Funk and J.W. Deaton. The interlaminar fracture toughness of woven graphite/epoxy composites. NASA Technical paper ADA309239, 1989. 
[9] S. L. Bazhenov. Strong bending in the DCB interlaminar test of thin, E-glass woven-fabric-reinforced laminates. Composites, 22(4):275-280, 1991.

[10] Bernard Schrauwen and Ton Peijs. Influence of matrix ductility and fibre architecture on the repeated impact response of glass-fibre-reinforced laminated composites. Applied Composite Materials, 9(6):331-352, 2002.

[11] P. T. Curtis and S. M. Bishop. An assessment of the potential of woven carbon fibre-reinforced plastics for high performance applications. Composites, 15(4):259-265, 1984.

[12] Vlot A and Jan Willem Gunnink. Fibre metal laminates: An introduction. Kluwer Academic Publishers, 2001.

[13] A. Vlot. Impact properties of fibre metal laminates. Composites Engineering, 3(10):911-927, 1993.

[14] M. Sadighi, R. C. Alderliesten, and R. Benedictus. Impact resistance of fibermetal laminates: A review. International Journal of Impact Engineering, 49(0):77-90, 2012.

[15] A. Vlot. Impact loading on fibre metal laminates. International Journal of Impact Engineering, 18(3):291-307, 1996.

[16] Allen J Fawcett. Boeing 787 - damage tolerance and the composite airframe. NIAR/FAA Workshop for Composite Damage Tolerance and Maintenance, 2006.

[17] A.T. Nettles and M...J. Douglas. A comparision of quasi static indentation testing to low velocity impact testing. Composite Materials: Testing,Design, and Acceptance Criteria, ASTM STP 1416, 2002.

[18] A Vlot. Low-velocity impact loading on fibre reinforced aluminium laminates (ARALL) and other aircraft sheet materials. Dissertation: Delft University of Technology, 1991. 
[19] Gin Boay Chai and Periyasamy Manikandan. Low velocity impact response of fibre-metal laminates - A review. Composite Structures, 107(0):363-381, 2014.

[20] Roman Starikov. Assessment of impact response of fiber metal laminates. International Journal of Impact Engineering, 59(0):38-45, 2013.

[21] A. Seyed Yaghoubi and B. Liaw. Thickness influence on ballistic impact behaviors of GLARE-5 fiber-metal laminated beams: Experimental and numerical studies. Composite Structures, 94(8):2585-2598, 2012.

[22] Yanxiong Liu and Benjamin Liaw. Effects of constituents and lay-up configuration on drop-weight tests of fiber-metal laminates. Applied Composite Materials, 17(1):43-62, 2009.

[23] R. Alderliesten, C. Rans, and R. Benedictus. The applicability of magnesium based fibre metal laminates in aerospace structures. Composites Science and Technology, 68(14):2983-2993, 2008.

[24] P. Corts and W. J. Cantwell. The impact properties of high-temperature fiber-metal laminates. Journal of Composite Materials, 41(5):613-632, 2007.

[25] A. Seyed Yaghoubi, Y. Liu, and B. Liaw. Stacking sequence and geometrical effects on low-velocity impact behaviors of GLARE-5 (3/2) fiber-metal laminates. Journal of Thermoplastic Composite Materials, 25(2):223-247, 2011.

[26] M. R. Abdullah and W. J. Cantwell. The impact resistance of polypropylene-based fibre-metal laminates. Composites Science and Technology, 66(1112):1682-1693, 2006.

[27] Shengqing Zhu and Gin Boay Chai. Low-velocity impact response of fibremetal laminates -Experimental and finite element analysis. Composites Science and Technology, 72(15):1793-1802, 2012. 
[28] P. Compston, W.J. Cantwell, C. Jones, and N.Jones. Impact perforation resistance and fracture mechanisms of a thermoplastic based fiber-metal laminate. Journal of material science letters, 20:597 599, 2001.

[29] G. Reyes and W.J. Cantwell. The mechanical properties of fiber-metal laminates based on glass fibre reinforced polypropylene. Composites Science and Technology, 60:1085-1094, 2000.

[30] R. R. Kulkarni, K. K. Chawla, U. K. Vaidya, M. C. Koopman, and A. W. Eberhardt. Characterization of long fiber thermoplastic/metal laminates. Journal of Materials Science, 43(13):4391-4398, 2008.

[31] J. F. Laliberte, C. Poon, P. V. Straznicky, and A. Fahr. Post-impact fatigue damage growth in fibermetal laminates. International Journal of Fatigue, 24(24):249-256, 2002.

[32] G. Caprino, G. Spataro, and S. Del Luongo. Low-velocity impact behaviour of fibreglass-aluminium laminates. Composites Part A: Applied Science and Manufacturing, 35(5):605-616, 2004.

[33] P. Corts and W. J. Cantwell. The fracture properties of a fibre-metal laminate based on magnesium alloy. Composites Part B: Engineering, 37(23):163-170, 2005.

[34] Jeremy Laliberte, Paul V. Straznicky, and Cheung Poon. Impact damage in fiber metal laminates, part 1: Experiment. AIAA Journal, 43(11):2445-2453, 2005.

[35] Qi Chen, Zhidong Guan, Zengshan Li, Zhaojie Ji, and Yue Zhuo. Experimental investigation on impact performances of GLARE laminates. Chinese Journal of Aeronautics, 28(6):1784-1792, 2015.

[36] G. D. Lawcock, L. Ye, Y. W. Mai, and C. T. Sun. Effects of fibre/matrix adhesion on carbon-fibre-reinforced metal laminates - II.Impact behaviour. Composites Science and Technology, 57(12):1621-1628, 1998. 
[37] Rohchoon Park and Jyongsik Jang. Effect of stacking sequence on the compressive performance of impacted aramid fiber/glass fiber hybrid composite. Polymer Composites, 21(2):231-237, 2000.

[38] C. Atas. An experimental investigation on the impact response of fiberglass/aluminum composites. Journal of Reinforced Plastics and Composites, 26(14):1479-1491, 2007.

[39] Guocai Wu, Jenn-Ming Yang, and H. T. Hahn. The impact properties and damage tolerance and of bi-directionally reinforced fiber metal laminates. Journal of Materials Science, 42(3):948-957, 2007.

[40] Hyoungseock Seo, H. T. Hahn, and Jenn-Ming Yang. Impact damage tolerance and fatigue durability of GLARE laminates. Journal of Engineering Materials and Technology, 130(4), 2008.

[41] J.G. Carrillo and W.J. Cantwell. Scaling effects in the low velocity impact response of fiber-metal laminates. Journal of Reinforced Plastics and Composites, 27(9):893-907, 2008.

[42] S. Mckown, W.J. Cantwell, and N. Jones. Investigation of scaling effects in fiber-metal laminates. Journal of Composite Materials, 42(9):865-888, 2008.

[43] F. Bagnoli, M. Bernabei, D. Figueroa-Gordon, and P. E. Irving. The response of aluminium/GLARE hybrid materials to impact and to in-plane fatigue. Materials Science and Engineering: A, 523(12):118-124, 2009.

[44] S. H. Song, Y. S. Byun, T. W. Ku, W. J. Song, J. Kim, and B. S. Kang. Experimental and numerical investigation on impact performance of carbon reinforced aluminum laminates. Journal of Materials Science and Technology, 26(4):327-332, 2010

[45] J. Fan, W.J Cantwell, and Z.W Guan. The low-velocity impact response of fiber-metal laminates. Journal of Reinforced Plastics and Composites, 30(1):26-35, 2011. 
[46] J.Y Fan, Z.W Guan, and W. J. Cantwell. Structural behaviour of fibre metal laminates subjected to a low velocity impact. Science China Physics, Mechanics and Astronomy, 54(6):1168-1177, 2011.

[47] J.Y Fan, Z.W Guan, and W. J. Cantwell. Numerical modelling of perforation failure in fibre metal laminates subjected to low velocity impact loading. Composite Structures, 93(9):2430-2436, 2011.

[48] G. R. Rajkumar, M. Krishna, H. N. Narasimha Murthy, S. C. Sharma, and K. R. Vishnu Mahesh. Experimental investigation of low-velocity repeated impacts on glass fiber metal composites. Journal of Materials Engineering and Performance, 21(7):1485-1490, 2011.

[49] Freddy D. Morinire, Ren C. Alderliesten, Mehdi Yarmohammad Tooski, and Rinze Benedictus. Damage evolution in GLARE fibre-metal laminate under repeated low-velocity impact tests. Central European Journal of Engineering, 2(4):603-611, 2012.

[50] M. Sadighi, T. Prnnen, R. C. Alderliesten, M. Sayeaftabi, and R. Benedictus. Experimental and numerical investigation of metal type and thickness effects on the impact resistance of fiber metal laminates. Applied Composite Materials, 19(3-4):545-559, 2011.

[51] A. Seyed Yaghoubi, Y. X. Liu, and B. M. Liaw. Drop-weight impact studies of GLARE 5 fiber-metal laminates. pages 267-279, 2011.

[52] N. Tsartsaris, M. Meo, F. Dolce, U. Polimeno, M. Guida, and F. Marulo. Low-velocity impact behavior of fiber metal laminates. Journal of Composite Materials, 45(7):803-814, 2011.

[53] T. Parnanen, R. Alderliesten, C. Rans, T. Brander, and O. Saarela. Applicability of AZ31B-H24 magnesium in fibre metal laminates - An experimental impact research. Composites Part A: Applied Science and Manufacturing, 43(9):1578-1586, 2012. 
[54] F. D. Morinire, R. C. Alderliesten, M. Sadighi, and R. Benedictus. An integrated study on the low-velocity impact response of the GLARE fibre-metal laminate. Composite Structures, 100(0):89-103, 2013.

[55] T. Parnanen, M. Kanerva, E. Sarlin, and O. Saarela. Debonding and impact damage in stainless steel fibre metal laminates prior to metal fracture. Composite Structures, 119:777-786, 2015.

[56] Serge Abrate. Impact on laminated composite materials. Applied Mechanics Reviews, 44(4):155-190, 1991.

[57] F. Hashagen, J. C. J. Schellekens, R. de Borst, and H. Parisch. Finite element procedure for modelling fibre metal laminates. Composite Structures, 32(14):255-264, 1995.

[58] J. Boer H. Carmone C Linde, P. Pleitner. Modelling and simulation of fibre metal laminates. ABAQUS Users Conference:2004, Boston,Massachusetts, 2004.

[59] P. Maimi, P. P. Camanho, J. A. Mayugo, and C. G. Dvila. A continuum damage model for composite laminates: Part I - constitutive model. Mechanics of Materials, 39(10):897-908, 2007.

[60] J. Laliberte and C. Poon. Numerical modeling of low velocity impact damage in fiber-metal laminates. Polymer Composites, pages 603-611, 2008.

[61] Z. W. Guan, W. J. Cantwell, and R. Abdullah. Numerical modeling of the impact response of fiber-metal laminates. Polymer Composites, 30(5):603-611, 2009.

[62] Hyoungseock Seo, Jake Hundley, H. T. Hahn, and Jeen-Ming Yang. Numerical simulation of glass-fiber-reinforced aluminum laminates with diverse impact damage. AIAA Journal, 48(3):676-687, 2010. 
[63] Hayato Nakatani, Tatsuro Kosaka, Katsuhiko Osaka, and Yoshihiro Sawada. Damage characterization of titanium/GFRP hybrid laminates subjected to low-velocity impact. Composites Part A: Applied Science and Manufacturing, 42(7):772-781, 2011.

[64] L. Iannucci. Progressive failure modelling of woven carbon composite under impact. International Journal of Impact Engineering, 32(6):1013-1043, 2006.

[65] ABAQUS user's manual (2014). ABAQUS.version.6.14.

[66] S. Timoshenko and S. Woinowsky-Krieger. Theory of plates and shells. McGraw-Hill, 1959.

[67] K. N. Shivakumar, W. Elber, and W. Illg. Prediction of low-velocity impact damage in thin circular laminates. AIAA Journal, 23(3):442-449, 1985.

[68] H. Babaei and A. Darvizeh. Analytical study of plastic deformation of clamped circular plates subjected to impulsive loading. Journal of mechanics of materials and structures, 7(4):309 - 322, 2012.

[69] George S. Bikakis George J. Tsamasphyros. Analytical modelling of circular GLARE laminated plates under lateral indentation. Advanced Composites Letters, 18(1), 2009.

[70] Serge Abrate. Modeling of impacts on composite structures. Composite Structures, 51(2):129-138, 2001.

[71] S. S. Pang, Y. Zhao, C. Yang, and S. A. Griffin. Impact response of composite laminates with a hemispherical indenter. Polymer Engineering Science, 31(20):1461-1466, 1991.

[72] K. Malekzadeh. Analytical prediction of low-velocity impact response of composite sandwich panels using new TDOF spring-mass-damper model. Journal of Composite Materials, 40(18):1671-1689, 2006. 
[73] Douglas S. Cairns and Paul A. Lagace. Transient response of graphite/epoxy and kevlar/epoxy laminates subjected to impact. AIAA Journal, 27(11):1590$1596,1989$.

[74] C. C. Foo, L. K. Seah, and G. B. Chai. A modified energy-balance model to predict low-velocity impact response for sandwich composites. Composite Structures, 93(5):1385-1393, 2011.

[75] Michael O. Pierson and Reza Vaziri. Analytical solution for low-velocity impact response of composite plates. AIAA Journal, 34(8):1633-1640, 1996.

[76] Ik-Hyeon Choi, In-Geol Kim, Seok-Min Ahn, and Chan-Hong Yeom. Analytical and experimental studies on the low-velocity impact response and damage of composite laminates under in-plane loads with structural damping effects. Composites Science and Technology, 70(10):1513-1522, 2010.

[77] Michelle S. Hoo Fatt, Chunfu Lin, Duane M. Revilock Jr, and Dale A. Hopkins. Ballistic impact of GLARE fibermetal laminates. Composite Structures, 61(12):73-88, 2003.

[78] George J. Tsamasphyros and George S. Bikakis. Analytical modeling to predict the low velocity impact response of circular GLARE fibermetal laminates. Aerospace Science and Technology, 2013.

[79] G. H. Payeganeh, F. Ashenai Ghasemi, and K. Malekzadeh. Dynamic response of fibermetal laminates (FMLs) subjected to low-velocity impact. Thin-Walled Structures, 48(1):62-70, 2010.

[80] R. Benedictus F.D. Morinire, R.C. Alderliesten. Energy distribution in GLARE and 2024-T3 aluminium during low-velocity impact. In 28th International Congress of the Aeronautical Sciences.

[81] Werner Goldsmith. Impact: The Theory and Physical Behaviour of Colliding Solids. Dover Publications, 1960. 
[82] Sun C. T. Tan, T. M. Wave propagation in graphite/epoxy laminates due to impact. NASA CR-168057, 1982.

[83] B. V. Sankar and C. T. Sun. An efficient numerical algorithm for transverse impact problems. Computers amp; Structures, 20(6):1009-1012, 1985.

[84] Ik Hyeon Choi and Cheol Ho Lim. Low-velocity impact analysis of composite laminates using linearized contact law. Composite Structures, 66(14):125-132, 2004.

[85] George J. Tsamasphyros and George S. Bikakis. Dynamic response of circular GLARE fibermetal laminates subjected to low velocity impact. Journal of Reinforced Plastics and Composites, 30(11):978-987, 2011.

[86] Chunfu Lin and Michelle S. Hoo Fatt. Perforation of composite plates and sandwich panels under quasi-static and projectile loading. Journal of Composite Materials, 40(20):1801-1840, 2006.

[87] M. N. Ghasemi Nejhad and A. Parvizi-Majidi. Impact behaviour and damage tolerance of woven carbon fibre-reinforced thermoplastic composites. Composites, 21(2):155-168, 1990.

[88] ASTM D6264, Standard test method for measuring the damage resistance of a fiber-reinforced polymer-matrix composite to a concentrated quasi-static indentation force. ASTM International, West Conshohocken, PA, 2012.

[89] ASTM D5420, Standard test method for impact resistance of flat, rigid plastic specimen by means of a striker impacted by a falling weight (gardener impact). ASTM International, West Conshohocken, PA, 2010.

[90] J.Simon A.F.Johnson. Modelling fabric reinforced composites under impact loads. In EUROMECH 400: Impact and Damage Tolerance Modelling of Composite Materials and Structures, 1999. 
[91] Christophe Bouvet, Bruno Castani, Matthieu Bizeul, and Jean-Jacques Barrau. Low velocity impact modelling in laminate composite panels with discrete interface elements. International Journal of Solids and Structures, 46(1415):2809-2821, 2009.

[92] P. P. Camanho, C. G. Davila, and M. F. de Moura. Numerical simulation of mixed-mode progressive delamination in composite materials. Journal of Composite Materials, 37(16):1415-1438, 2003.

[93] Gordon R. Johnson and William H. Cook. Fracture characteristics of three metals subjected to various strains, strain rates, temperatures and pressures. Engineering Fracture Mechanics, 21(1):31-48, 1985.

[94] Bazant Z.P and Oh B.H. Crack band theory for fracture of concrete. Materials and Structures, 16(3):155-177, 1983.

[95] ASTM E8, Standard test method for tension testing of metallic materials. ASTM International, West Conshohocken, PA, 2009.

[96] Chang Hee Suh, Yun-Chul Jung, and Young Suk Kim. Effects of thickness and surface roughness on mechanical properties of aluminum sheets. Journal of Mechanical Science and Technology, 24(10):2091-2098, 2010.

[97] L. V. Raulea, A. M. Goijaerts, L. E. Govaert, and F. P. T. Baaijens. Size effects in the processing of thin metal sheets. Journal of Materials Processing Technology, 115(1):44-48, 2001.

[98] T. P. Vo, Z. W. Guan, W. J. Cantwell, and G. K. Schleyer. Modelling of the low-impulse blast behaviour of fibremetal laminates based on different aluminium alloys. Composites Part B: Engineering, 44(1):141-151, 2013.

[99] M. V. Donadon, L. Iannucci, B. G. Falzon, J. M. Hodgkinson, and S. F. M. de Almeida. A progressive failure model for composite laminates subjected to low velocity impact damage. Computers Structures, 86(1112):1232-1252, 2008. 
[100] Jean Lemaitre. A Course on Damage Mechanics. Springer Berlin Heidelberg, 1996.

[101] P. Maimi, P. P. Camanho, J. A. Mayugo, and C. G. Dvila. A continuum damage model for composite laminates: Part II - Computational implementation and validation. Mechanics of Materials, 39(10):909-919, 2007.

[102] E. V. Gonzlez, P. Maim, P. P. Camanho, A. Turon, and J. A. Mayugo. Simulation of drop-weight impact and compression after impact tests on composite laminates. Composite Structures, 94(11):3364-3378, 2012.

[103] ASTM 3039, Standard test method for tension properties of matrix composite materials. ASTM International, West Conshohocken, PA, 2008.

[104] ASTM 3518, Standard test method for in-plane response of polymer matrix composite materials by tensile test of a 45 laminate. ASTM International, West Conshohocken, PA, 2007.

[105] S. T. Pinho, P. Robinson, and L. Iannucci. Fracture toughness of the tensile and compressive fibre failure modes in laminated composites. Composites Science and Technology, 66(13):2069-2079, 2006.

[106] Y. Mohammed, Mohamed K. Hassan, Abu El-Ainin H, and A. M. Hashem. Fracture properties of glass fiber composite laminates and size effect. Scholars Journal of Engineering and Technology, 1(1):13-26, 2013.

[107] A. Hillerborg, M. Moder, and P. E. Petersson. Analysis of crack formation and crack growth in concrete by means of fracture mechanics and finite elements. Cement and Concrete Research, 6(6):773-781, 1976.

[108] L. Daudeville, O. Allix, and P. Ladevze. Delamination analysis by damage mechanics: Some applications. Composites Engineering, 5(1):17-24, 1995. 
[109] Z. Zou, S. R. Reid, S. Li, and P. D. Soden. Modelling interlaminar and intralaminar damage in filament-wound pipes under quasi-static indentation. Journal of Composite Materials, 36(4):477-499, 2002.

[110] Davila Carlos, Camanho Pedro, and Moura Marcelo de. Mixed-mode decohesion elements for analyses of progressive delamination. American Institute of Aeronautics and Astronautics, 2001.

[111] A. Turon, C. G. Dvila, P. P. Camanho, and J. Costa. An engineering solution for mesh size effects in the simulation of delamination using cohesive zone models. Engineering Fracture Mechanics, 74(10):1665-1682, 2007.

[112] G. Alfano and M. A. Crisfield. Finite element interface models for the delamination analysis of laminated composites: mechanical and computational issues. International Journal for Numerical Methods in Engineering, 50(7):1701-1736, 2001.

[113] B. R. K. Blackman, A. J. Kinloch, F. S. Rodriguez-Sanchez, and W. S. Teo. The fracture behaviour of adhesively-bonded composite joints: Effects of rate of test and mode of loading. International Journal of Solids and Structures, 49(13):1434-1452, 2012.

[114] Herzl Chai. Shear fracture. International Journal of Fracture, 37:137-159, 1988.

[115] ASTM D3433, Standard test method for fracture strength in cleavage of adhesives in bonded metal joints. ASTM International, West Conshohocken, PA, 2012.

[116] ASTM D5528, Standard test method for mode i interlaminar fracture toughness of unidirectional fiber-reinforced polymer matrix composites. ASTM International, West Conshohocken, PA, 2013.

[117] JF.Laliberte. Investigation of low-velocity impact damage in fibre-metallaminates. Thesis, Carleton University, 2002. 
[118] A. Vlot and J. W. Van Ingen. Delamination resistance of post-stretched fibre metal laminates. Journal of Composite Materials, 32(19):1784-1805, 1998.

[119] James R. Reeder, Kevin Demarco, and Karen S. Whitley. The use of doubler reinforcement in delamination toughness testing. Composites Part A: Applied Science and Manufacturing, 35(11):1337-1344, 2004.

[120] M. D. Thouless, J. L. Adams, M. S. Kafkalidis, S. M. Ward, R. A. Dickie, and G. L. Westerbeek. Determining the toughness of plastically deforming joints. Journal of Materials Science, 33(1):189-197, 1998.

[121] Kyongchan Song, Carlos G Dvila, and Cheryl A Rose. Guidelines and parameter selection for the simulation of progressive delamination. Abaqus User Conference, Newport, 2008.

[122] S. Mostovoy, E. J. Ripling, and C. F. Bersch. Fracture toughness of adhesive joints. The Journal of Adhesion, 3(2):125-144, 1971.

[123] B. Blackman, J. P. Dear, A. J. Kinloch, and S. Osiyemi. The calculation of adhesive fracture energies from double-cantilever beam test specimens. Journal of Materials Science Letters, 10(5):253-256, 1991.

[124] R. Klement, S. Rolc, R. Mikulikova, and J. Krestan. Transparent armour materials. Journal of the European Ceramic Society, 28(5):1091-1095, 2008.

[125] H. P. Kirchner and R. M. Gruver. Localized impact damage in glass. Materials Science and Engineering, 28(1):153-160, 1977.

[126] Y. M. Tsai and H. Kolsky. A study of the fractures produced in glass blocks by impact. Journal of the Mechanics and Physics of Solids, 15(4):263-278, 1967.

[127] G. F. Raiser, J. L. Wise, R. J. Clifton, D. E. Grady, and D. E. Cox. Plate impact response of ceramics and glasses. Journal of Applied Physics, 75(8):3862$3869,1994$. 
[128] Dennis B. Barr Joyce L. Illinger, Robert W. Lewis. Effects of adhesive structure on resistance and optical properties acrylic/polycarbonate laminates. Adhesion Science and Technology, 9:217-32, 1975.

[129] Joshua S. Stenzler and N. C. Goulbourne. The effect of polyacrylate microstructure on the impact response of PMMA/PC multi-laminates. International Journal of Impact Engineering, 38(7):567-576, 2011.

[130] A. Ball. The low velocity impact behaviour of glass-polymer laminated plates. J. Phys. IV France, 07(C3):C3-921-C3-926, 1997.

[131] Yanxiong Liu and Benjamin Liaw. Drop-weight impact tests and finite element modeling of cast acrylic/aluminum plates. Polymer Testing, 28(8):808-823, 2009.

[132] Yanxiong Liu and Benjamin Liaw. Drop-weight impact tests and finite element modeling of cast acrylic plates. Polymer Testing, 28(6):599-611, 2009.

[133] Wenke Hu, Yenan Wang, Jian Yu, Chian-Fong Yen, and Florin Bobaru. Impact damage on a thin glass plate with a thin polycarbonate backing. International Journal of Impact Engineering, 62(0):152-165, 2013.

[134] D.C. Prevorsek, H.B. Chin, and A. Bhatnagar. Damage tolerance: design for structural integrity and penetration. Composite Structures, 23(2):137 - 148, 1993.

[135] M. V. Hosur, M. Adbullah, and S. Jeelani. Studies on the low-velocity impact response of woven hybrid composites. Composite Structures, 67(3):253-262, 2005.

[136] Jang-Kyo Kim, Man-Lung Sham, Min-Seok Sohn, and Hiroyuki Hamada. Effect of hybrid layers with different silane coupling agents on impact response of glass fabric reinforced vinylester matrix composites. Polymer, 42(17):74557460, 2001. 
[137] Metin Sayer, Numan B. Bekta, and Onur Sayman. An experimental investigation on the impact behavior of hybrid composite plates. Composite Structures, 92(5):1256-1262, 2010.

[138] F. D. Morinire, R. C. Alderliesten, and R. Benedictus. Low-velocity impact energy partition in GLARE. Mechanics of Materials, 66(0):59-68, 2013.

[139] R.L Woodward and S.J Cimpoeru. A study of the perforation of aluminium laminate targets. International Journal of Impact Engineering, 21(3):117 $131,1998$.

[140] E. V. Gonzlez, P. Maim, P. P. Camanho, C. S. Lopes, and N. Blanco. Effects of ply clustering in laminated composite plates under low-velocity impact loading. Composites Science and Technology, 71(6):805-817, 2011.

[141] Edgar Fuoss, Paul V. Straznicky, and Cheung Poon. Effects of stacking sequence on the impact resistance in composite laminates part 1: parametric study. Composite Structures, 41(1):67-77, 1998.

[142] Karlsson Hibbitt and Sorensen. ABAQUS: Theory Manual. Hibbitt, Karlsson Sorensen, 1997.

[143] Periyasamy Manikandan and Gin Boay Chai. A layer-wise behavioral study of metal based interply hybrid composites under low velocity impact load. Composite Structures, 117(0):17-31, 2014.

[144] L. Falk, M., A. Needleman, and R. Rice, J. A critical evaluation of cohesive zone models of dynamic fracture, volume 11. 2001. 


\section{Appendix A}

\section{Selection of cohesive analysis pa-}

\section{rameters}

The methodology proposed by Turon et.al [111 to select optimum analysis parameter and mesh size for accurate modelling of delamination progression using cohesive zone model is narrated in the present appendix. ABAQUS cohesive element (COH3D8) of different in-plane length is used to simulate progressive delamination growth in Mode-I fracture simulation with dissimilar DCB substrate. The coarsest mesh size with minimum iterations required to simulate accurate delamination propagation among them is outlined.

\section{A.1 Interfacial strength modification strategy}

For an accurate simulation of delamination progression, the two cohesive parameters that need to be optimized for a given interface strength $\left(t_{\alpha}^{0}\right)$ and critical fracture toughness $\left(G_{\alpha}^{c}\right)$ are: (i) Cohesive zone length $l_{c z}$ and (ii) number of elements in cohesive zone $N_{e}$.

Cohesive zone length is defined as the distance from crack front to the integration point where the specified $\left(t_{\alpha}^{0}\right)$ is attained as shown in Figure A.1.For any fracture mode, the $l_{c z}$ can be expressed in the form 107:

$$
l_{c z}=M E \frac{G_{\alpha}^{c}}{t_{\alpha}^{0}}
$$




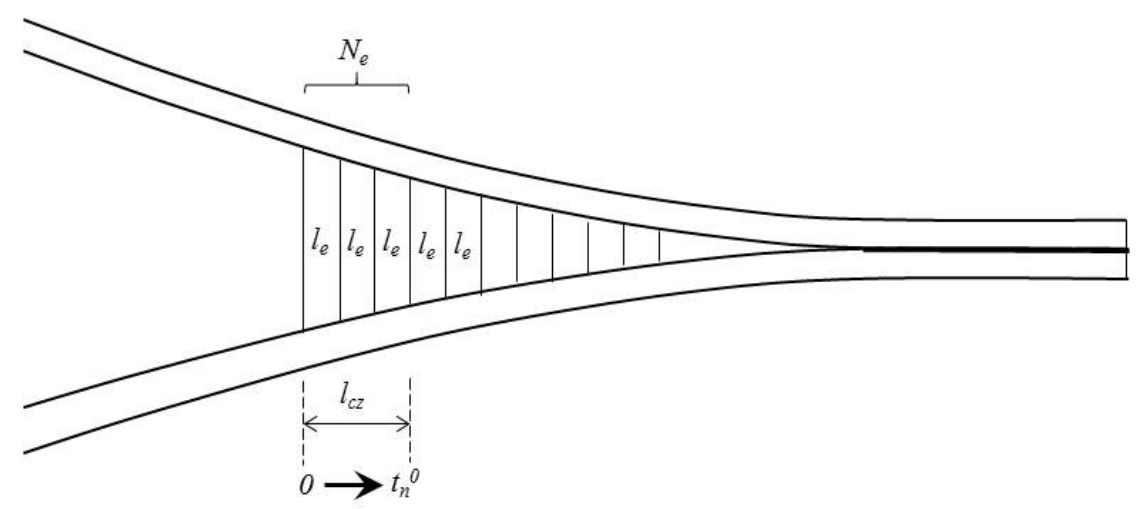

Figure A.1: Geometry parameters of cohesive element necessary to model crack propagation

and the number of cohesive finite elements $N_{e}$ corresponds to $l_{c z}$ is given by:

$$
N_{e}=\frac{l_{c z}}{l_{e}}
$$

where $E$ is the young's modulus of interface material, $l_{e}$ is the mesh size along the direction of crack growth and $M$ is the constant cohesive parameter ranges from 0.21 to 1 . In the present work, $M$ takes a value of 1 [107].

In literature, the minimum requirement of $N_{e}$ in $l_{c z}$ is not well established and ranges widely from 1 to 8 depending on type of bonded substrate material [110,144. In the case of FRP materials, typical range of $l_{c z}$ is smaller than $1 \mathrm{~mm}$ and requires extremely fine mesh along the interlaminar region to simulate delamination. This leads to high computational cost even for small prototype models.

On altering the $t_{\alpha}^{0}$, it is possible to lengthen $l_{c z}$ artificially for a given mesh size [111]. This allows delamination to be simulated using cohesive elements even at reasonable coarse mesh [121]. To accomplish that, the adjusted $t_{\alpha}^{a}$ can be expressed for a given $N_{e}$ and $l_{e}$ by combining the equation $(\mathrm{A} .1)$ and $\mathrm{A} .2$ which leads to:

$$
\bar{t}_{\alpha}^{a}=\sqrt{\frac{E G_{\alpha}^{c}}{N_{e} l_{e}}}
$$




\section{A.2 Analysis of Mode-I progressive delamination growth}

The reliability of the above interfacial strength modification strategy is confirmed through mode-I DCB fracture simulation as presented in section 3.5.2.3. From equation A.1, the $l_{c z}$ for mode-I fracture yields $0.26 \mathrm{~mm}$. Mesh parameters of different $l_{e}\left(\geq l_{c z}\right)$ and $N_{e}$ are considered for mesh sensitivity investigation. Details of mesh parameters and corresponding adjusted interfacial strength estimated using equation A.3 is tabulated in Table A.1.

Table A.1: Adjusted normal interfacial strength with respect to different mesh size.

\begin{tabular}{c|c|c|c|c|c|c}
\hline \multirow{2}{*}{$N_{e}$} & \multicolumn{2}{|c|}{$l_{e}=1 \mathrm{~mm}$} & \multicolumn{2}{c|}{$l_{e}=0.5 \mathrm{~mm}$} & \multicolumn{2}{c}{$l_{e}=0.3 \mathrm{~mm}$} \\
\cline { 2 - 6 } & $t_{\alpha}^{a}(\mathrm{MPa})$ & $l_{c z}(\mathrm{~mm})$ & $t_{\alpha}^{a}(\mathrm{MPa})$ & $l_{c z}(\mathrm{~mm})$ & $t_{\alpha}^{a}(\mathrm{MPa})$ & $l_{c z}(\mathrm{~mm})$ \\
\hline 1 & 25.5 & 1.0 & 36.1 & 0.5 & 45.8 & 0.3 \\
3 & 14.7 & 3.0 & 20.8 & 1.5 & 26.4 & 0.9 \\
5 & 11.4 & 5.0 & 16.1 & 2.5 & 20.5 & 1.5 \\
8 & 9.0 & 8.0 & 12.8 & 4.0 & 16.2 & 2.4 \\
\hline
\end{tabular}

The load-deflection responses obtained for different mesh size $l_{e}$ are shown in Figure A.2. Irrespective of $l_{e}$, the response predicted by adjusted interfacial strength $t_{\alpha}^{a}$ with $N_{e}=1$ did not converge and no numerical solution exists. For all other cases, the predicted softening crack propagation response is almost similar compared to the experiment measurement with some minor variations.

For the mesh size of $l_{e}=1 \mathrm{~mm}$, the softening response shows spurious oscillations for smaller $N_{e}$ which means the mesh size is too coarse to accurately predict the crack propagation. However, the amplitude of oscillation is somewhat attenuated with an increase in $N_{e}$. It can be seen that responses obtained with finer mesh size of $l_{e}=0.5 \mathrm{~mm}$ and $l_{e}=0.3 \mathrm{~mm}$ over predicts the peak load as shown in Figure A.2(b) and A.2(c). Meanwhile for this mesh size, the softening response exhibit smooth crack propagation. On comparing the number of iterations required to complete the solution, mesh sizes with more $N_{e}$ in its cohesive zone length $l_{c z}$ was shown to be computationally efficient.

The above comparative study confirms that the interface strength $t_{\alpha}^{0}$ required to 
a)

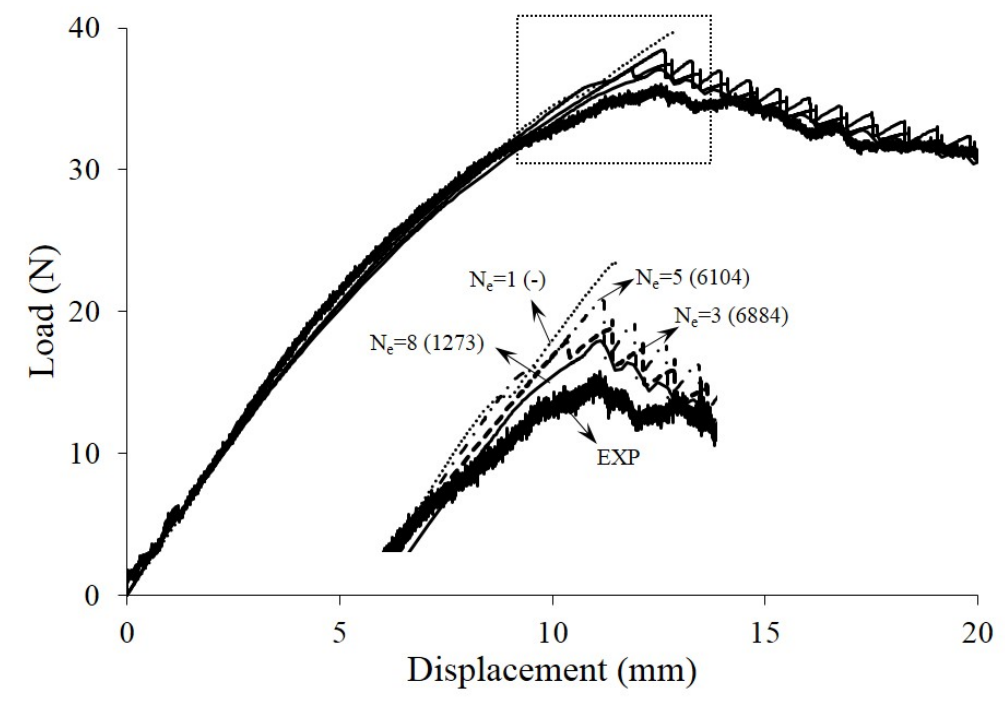

b)

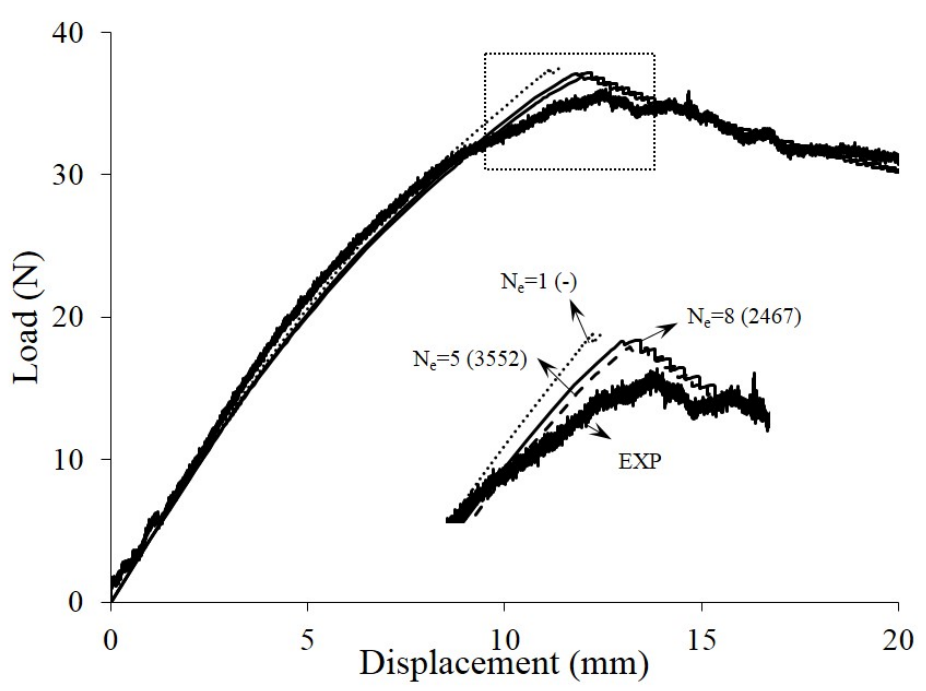

c)

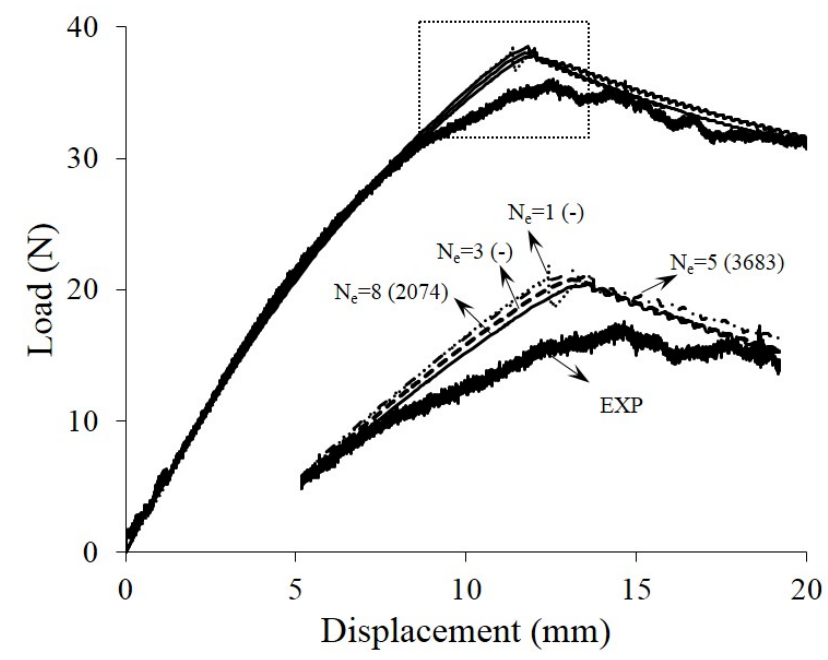

Figure A.2: Load-displacement curve of DCB sample with different mesh size a) $l_{e}=1 \mathrm{~mm} \mathrm{b)} l_{e}=0.5 \mathrm{~mm} \mathrm{c)} l_{e}=0.3 \mathrm{~mm}$. Note: Number inside the parentheses are number of iterations required to complete the solution 
initiate crack propagation does not have significant influence on the overall response. Thus, interface strength modification strategy can be used to model accurate crack propagation using cohesive elements at coarse mesh. For the present comparative study of DCB simulation, solution obtained using lowest adjusted interfacial strength $t_{\alpha}^{a}$ with larger $N_{e}\left(l_{e}=1 m m \& N_{e}=8\right)$ yields optimum solution and result is efficient in accordance with mesh size and computational power. All the simulations in the present work are structured based on the above predicted optimum mesh size. 